\title{
A Fatorização de Stein \\ e o Número de Singularidades de Aplicações Estáveis
}

\author{
Jorge Tadashi Hiratuka
}

TESE APRESENTADA

$\mathrm{AO}$

INSTITUTO DE MATEMÁTICA E ESTATÍSTICA

DA

UNIVERSIDADE DE SÃO PAULO
PARA OBTENÇÃO DO GRAU DE DOUTOR EM

MATEMÁTICA

\author{
Área de Concentração: Singularidades \\ Orientador: Prof. Dr. Osamu Saeki \\ Co-orientadora: Profa. Dra. Dirce K. Hayashida
}

-São Paulo, Outubro de 2001- 


\section{A Fatorização de Stein e o Número de Singularidades de Aplicações Estáveis}

Este exemplar corresponde à redação final da tese devidamente corrigida e defendida por Jorge Tadashi Hiratuka e aprovada pela comissão julgadora.

- São Paulo, 19 de novembro de 2001. -

Banca examinadora:

- Profa. Dra. Dirce Kiyomi Hayashida (co-orientadora) (UFSCar)

- Prof. Dr. Washington Luiz Marar (ICMC-USP)

- Prof. Dr. Oziride Manzoli Neto (ICMC-USP)

- Prof. Dr. Paulo Ferreira da Silva Porto Junior (ICMC-USP)

- Prof. Dr. Brasil Terra Leme (FAFI) 
Dedico à minha mãe (in memoriam), que dedicou até o último instante de sua vida em prol de seus filhos;

e ao Dr. Daisaku Ikeda, por ter me ensinado a maneira mais nobre e plena de se viver. 
O inverno nunca falha em se tornar primavera!

Nitiren Daishonin 
"Não faz mal que seja pouco, o que importa é que o avanço de hoje seja maior que o de ontem. Que nossos passos de amanhã sejam mais largos que os de hoje."

"A perseverança é de vital importância. A falta de persistência, superficialidade e uma atitude insincera só resultarão na frustação de seus empreendimentos. É importante continuar a meditar e a desafiar o desconhecido."

"As pessoas que empreendem verdadeiras ações são sempre capazes de provar sua grandeza a despeito de quaisquer críticas que são lançadas em seus caminhos. Ações sérias também atraem uma simpática atenção das pessoas com verdadeiros corações."

"Não desistam até o fim, para tanto, a paciência, o esforço e a perseverança é que servem para auxiliar-nos a lutar. Esta é a verdadeira grandiosidade dos seres humanos."

"O indivíduo dever superar todas as dificuldades e viver de acordo com seus nobres ideais."

"As ondas tornam-se mais bravias todas as vezes em que encontram um obstáculo."

"Um verdadeiro herói é aquele que é forte quando levanta-se sozinho." 


\section{Agradecimentos}

Gostaria primeiramente de expressar a minha mais profunda gratidão e carinho à Dona Célia (in memoriam), um exemplo de mãe, que, levada prematuramente pelo sono eterno da vida, não pode, por causa de apenas algumas semanas, ver concluída mais esta etapa de minha existência. Sem ela eu não teria galgado nem o primeiro degrau de minha vida acadêmica. Agradeço, também, ao Prof. Dr. Osamu Saeki, pela intensa dedicação e amizade, por ter acreditado no meu trabalho e que, mesmo à distância, não poupou esforços para orientar-me. À Profa. Dra. Dirce Hayashida, pela amizade, co-orientação, incentivos constantes e que, na sua mais pura benevolência, me fez reerguer das cinzas para concluir este trabalho. Ao Dr. Daisaku Ikeda, que, equilibrando severas orientações com compreensão e amizade, levou-me a desafiar meu próprio limite e a crescer como um ser humano digno. Aos meus amigos, Daciberg Gonçalves, Pedro Fagundes e Antonio Geloneze pelas valorosas sugestões e conversas. Aos Professores e colegas do IME-USP, pela minha formação. Aos meus familiares. Aos meus amigos, Cristina Bonomi, Maria Eiko, Alice Takada, Elizabeth Santos, Henrique Guzzo e família, Gladys Chalom, Margarete Poli e família, Celia Manabe, Sandra Lopes, Francisca Silva, Sueli Dian, Rosária Borges, Vera Ribeiro, Joelma Gomes, Justina Bueno e Marcelo Modesto pelos incentivos e torcida. Aos amigos que deixei na Inglaterra, Robert Reynolds, Peter Lloyd, Ann Murray, Jeffrey Clarke e tantos outros, pela amizade e carinho. Aos dirigentes da BSGI, em particular, à Dona Dirce Ivamoto, que, com toda a rigorosidade e benevolência, ajudaram a construir um sólido alicerce em minha vida. À Fernanda Cardona pelas dicas do Latex, pelo auxílio nas correções da tese e, principalmente no final, quando, junto com a Elisa Takada, foram o meu ponto de apoio para continuar caminhando, mesmo tendo o coração partido por uma incomensurável perda. À CAPES, pela bolsa de estudos na Inglaterra. Enfim, a todos aqueles, injustamente não citados, que de alguma forma contribuíram para a conclusão deste trabalho. Por fim, agradeço àqueles que, de alguma forma, foram um obstáculo nesta jornada, pois apenas contribuíram para que eu pudesse forjar ainda mais o meu

espírito. A eles só tenho uma coisa a dizer: "Eu venci!" Todo esse esforço seria vão se ao final não me restasse nenhum espírito de gratidão. 


\section{Resumo}

Neste trabalho mostramos o seguinte resultado: "Seja $g: N^{n} \rightarrow P^{p}$ uma aplicação contínua entre espaços topológicos. Suponhamos que $N^{n} \mathrm{e}$ $P^{p}$ sejam localmente compactos e $g$ é própria. Se $g$ é triangulável, então a fatorização de Stein de $g$ também é triangulável." Verificamos ainda que a triangulação de $\bar{g}: W_{g} \rightarrow P^{p}$, dado pela fatorização de Stein de $g$, é sempre não-degenerada. O estudo das singularidades simpliciais da triangulação de $\bar{g}$ nos dá informações importantes sobre as singularidades da aplicação $g$. Quando $g$ é uma aplicação topologicamente estável entre variedades, desenvolvemos algumas fórmulas, para os casos $(n, p)=(3,2)$ e $(n, p)=(4,3)$, nas quais associamos invariantes topológicos ao número de singularidades destas aplicações. Como conseqüência, dentre outros, provamos o bem conhecido resultado "Toda variedade fechada tridimensional tem característica de Euler nula", sem utilizar a dualidade de Poincaré; para aplicações estáveis, $N^{4} \rightarrow \mathbb{R}^{3}$, apresentamos algumas congruências (mod 2) entre o número de singularidades, por exemplo, verificamos que o número de rabos de andorinha definidos (veja definição na Proposição 6.1.1) é congruente $(\bmod 2)$ ao número de rabos de andorinha indefinidos. 


\begin{abstract}
Let $g: M^{n} \rightarrow N^{p}$ be a continuous map between topological spaces. Suppose $N^{n}$ and $P^{p}$ are locally compact and $g$ is proper. If $g$ is triangulable than its Stein factorization $W_{g}$ is a polyhedron. Also, the triangulation of $\bar{g}: W_{g} \rightarrow N$, given by the Stein factorization of $g$, is always non-degenerated. The study of simplicial singularities of the triangulation of $\bar{g}$ yields important information about the singularities of $g$ itself. When $g$ is a topologically stable map between manifolds we are able to develop some formulas for $(n, p)=(3,2)$ and $(n, p)=(4,3)$, where we associate topological invariants to the number of singularities of those maps. As a consequence, we prove the well-known result: "Every closed 3-dimensional manifold has null Euler characteristic.", without resorting to Poincaré duality. Moreover, for stable maps, $N^{4} \rightarrow \mathbb{R}^{3}$, we show some congruences ( $\left.\bmod 2\right)$ between the number of its singularities, por instance, we verify that the number of definite swallowtails (see definition in Proposição 6.1.1) is congruent (mod 2) to the number of indefinite swallowtails.
\end{abstract}




\section{Sumário}

$\begin{array}{ll}\text { Introdução } & 1\end{array}$

1 A fatorização de Stein e sua triangulação 5

1.1 Complexo simplicial geométrico . . . . . . . . . . . . 5

1.2 Complexo simplicial abstrato . . . . . . . . . . . . 12

1.3 A fatorização de Stein e sua triangulação . . . . . . . . . . . 14

1.4 Aplicações $\mathcal{C}^{\infty}$ trianguláveis . . . . . . . . . . . . . . . . 18

2 Um estudo sobre aplicações simpliciais $\quad 21$

2.1 O "join" entre subconjuntos do $\mathbb{R}^{n}$. . . . . . . . . . . . . . . 22

2.2 Alguns resultados sobre as fibras de uma aplicação simplicial entre simplexos . . . . . . . . . . . . . . 27

2.3 Um estudo sobre as fibras de uma aplicação simplicial entre complexos . . . . . . . . . . . . . . 35

2.4 Lemas sobre imagens inversas de simplexos . . . . . . . . . . 46

3 A fatorização de Stein de uma aplicação simplicial é triangulável

3.1 Exemplos ....................... 52

3.2 Complexo simplicial correspondente à fatorização de Stein . 59

3.3 Demonstração do Teorema 1.3.8 . . . . . . . . . . . . 75

4 Característica de Euler $\quad 81$

4.1 Característica de Euler . . . . . . . . . . . . . . . 81

4.2 Índices locais para aplicações simpliciais . . . . . . . . . . . . . . 83

4.3 Característica combinatória de Euler . . . . . . . . . . . . . 87 
5 Fórmulas referentes a aplicações estáveis de uma 3-variedade no $\mathbb{R}^{2}$

5.1 Triangulação da fatorização de Stein . . . . . . . . . . . . . 91

5.2 O número de singularidades de uma aplicação $g: N^{3} \rightarrow \mathbb{R}^{2}$. . 98

5.3 O índice $\operatorname{Ind}_{f}^{*}(v)$ e aplicações . . . . . . . . . . . 106

6 Fórmulas referentes a aplicações estáveis de uma 4-variedade no $\mathbb{R}^{3}$

6.1 Estudo local de aplicações $N^{4} \rightarrow \mathbb{R}^{3}$ estáveis . . . . . . . . 116

6.2 Uma fórmula sobre o número de singularidades . . . . . . . 120

6.3 O índice $\operatorname{Ind}_{f}^{*}(v)$ e o número de singularidades . . . . . . . 133

Apêndice

A As componentes conexas das fibras de $N^{3} \rightarrow \mathbb{R}^{2}$

B As características de Euler das fibras de $N^{3} \rightarrow \mathbb{R}^{2}$

C As componentes conexas das fibras de $N^{4} \rightarrow \mathbb{R}^{3}$

D As características de Euler das fibras de $N^{4} \rightarrow \mathbb{R}^{3}$ 


\section{Introdução}

Em 1955, H. Whitney [Wh] provou que uma aplicação $\mathcal{C}^{\infty}$ excelente $f: \mathbb{R} P^{2} \rightarrow \mathbb{R}^{2}$, do plano projetivo sobre o plano real, tem um número ímpar de cúspides. No mesmo ano, R. Thom [Th] mostrou que o número de cúspides deve ter a mesma paridade da característica de Euler $\chi\left(N^{2}\right)$ para uma aplicação excelente $f: N^{2} \rightarrow \mathbb{R}^{2}$, onde $N^{2}$ é uma superfície fechada. Desde então, o interesse na contagem das singularidades de uma aplicação tem motivado intensas pesquisas. Nessa direção, citamos, por exemplo, [Tu], [Ba], [Qu] e [FI] (veja resumo histórico na Introdução de [Ge2]) que obtiveram resultados análogos para aplicações entre superfícies. Mais recentemente, em [IM1], [IM2], [IM3], Izumiya \& Marar obtiveram uma fórmula onde mostram que a metade do número de "cross caps" mais o número de pontos triplos de aplicações genéricas de uma superfície fechada $N^{2}$ em uma variedade tridimensional $P^{3}$ é igual a $\chi(\operatorname{Im}(f))-\chi\left(N^{2}\right)$. Também, vários autores, como Ballesteros \& Saeki [BS], Geloneze [Ge1], Geloneze \& Morris [GM] e Houston [Ho], têm estudado índices para os vértices de uma aplicação simplicial com o objetivo de relacioná-los com invariantes topológicos dos espaços envolvidos.

Por outro lado, a fatorização de Stein $W_{g}$ de uma aplicação contínua $g: N^{n} \rightarrow P^{p}$, onde $N^{n}$ e $P^{p}$ são variedades de $n$ e $p$ as suas dimensões, respectivamente, é o espaço quociente, $N^{n} / \sim$, sob a relação de equivalência que identifica os pontos de uma mesma componente conexa das fibras de $g$. 
O conhecimento de $W_{g}$ fornece informações importantes sobre a estrutura da aplicação $g$ e possibilita criar um elo entre as singularidades de $g$ e invariantes topológicos dos espaços envolvidos. Também, usando essencialmente a fatorização de Stein, alguns autores, como Burlet \& de Rham [BdR], no caso $n=3$ e $p=2$, e Saeki [Sa1], no caso $n \geq p$, conseguiram caracterizar as variedades fechadas que admitem aplicações genéricas especiais.

Em [KLP], Kushner, Levine \& Porto mostraram que se $g: N^{3} \rightarrow \mathbb{R}^{2}$ é topologicamente estável e própria, então $W_{g}$ é triangulável. Posteriormente, em [PF], Porto \& Furuya provaram o mesmo resultado para uma aplicação $g: N^{4} \rightarrow \mathbb{R}^{2}$ e, em 1996, Kobayashi \& Saeki em [KS] generalizaram o resultado para uma aplicação $g: N^{n} \rightarrow \mathbb{R}^{2}$.

Nossos objetivos nessa tese são:

- mostrar que, sob as mesmas condições, se $g: N^{n} \rightarrow \mathbb{R}^{p}$, então $W_{g}$ é homeomorfa a um poliedro;

- como aplicação do resultado anterior, obter fórmulas, para os casos $(n, p)=(3,2)$ e $(n, p)=(4,3)$, nas quais associamos invariantes topológicos ao número de singularidades de $g$;

- obter fórmulas similares aos citados acima, porém sem utilizar a fatorização de Stein.

Apresentamos nosso trabalho da seguinte maneira:

No Capítulo 1, enunciamos conceitos básicos de complexos simpliciais geométricos e abstratos, bem como definições e propriedades de aplicações simpliciais. Definimos, também, a fatorização de Stein para uma aplicação contínua, a sua triangulação e enunciamos um dos resultados principais deste trabalho (Teorema 1.3.8), onde apresentamos condições suficientes para 
que a fatorização de Stein de uma aplicação contínua seja triangulável. Na última seção, enunciamos e utilizamos um resultado de Verona [Ve1], [Ve2], para concluir que a fatorização de Stein de toda aplicação diferenciável, topologicamente estável e própria entre variedades, também é triangulável.

No Capítulo 2, dada uma aplicação simplicial $f: K \rightarrow L$ entre complexos simpliciais, estudamos as fibras $|f|^{-1}(y)$ da aplicação $|f|:|K| \rightarrow|L|$ entre poliedros, onde $y \in|L|$. Tal estudo é necessário para mostrarmos que a fatorização de Stein $W_{|f|}$ (Definição 1.3.1) é um poliedro. De fato, provamos que se $\sigma \in f(K)$ é um simplexo tendo $b_{\sigma}$ como baricentro, então $\mid f \|_{|f|^{-1}(\stackrel{\circ}{\sigma})}$ é um fibrado trivial com fibra $|f|^{-1}\left(b_{\sigma}\right)$.

No Capítulo 3, provamos o seguinte resultado: "A fatorização de Stein de qualquer aplicação simplicial própria entre complexos simpliciais localmente finitos é um poliedro." Utilizando o resultado de Verona [Ve1], [Ve2] que garante ser, toda aplicação topologicamente estável e própria, triangulável, obtemos como corolário que a fatorização de Stein de toda aplicação $\mathcal{C}^{\infty}$ topologicamente estável e própria entre variedades sem bordo é homeomorfa a um poliedro. Em outras palavras, se $f: K \rightarrow L$ é uma triangulação de uma tal aplicação, a fatorização de Stein de $|f|$ define duas aplicações $q_{|f|}$ : $|K| \rightarrow W_{|f|}$ e $\overline{|f|}: W_{|f|} \rightarrow|L|$ e, assim, mostramos que $W_{|f|}$ é homeomorfo a um poliedro e que $\overline{|f|}$ é sempre não-degenerada.

No Capítulo 4, damos a definição da característica de Euler para complexos simpliciais e para seus espaços subjacentes; decompomos o índice de Ballesteros \& Saeki [BS] através do diagrama de Stein e, finalmente, definimos a característica combinatória de Euler. Esta última nos é muito útil para associar um invariante topológico a conjuntos não fechados. 
No Capítulo 5, aplicando o Teorema 1.3.8, obtemos fórmulas que relacionam as singularidades de uma aplicação $\mathcal{C}^{\infty}$-estável, $g: N^{3} \rightarrow \mathbb{R}^{2}$, onde $N^{3}$ é uma variedade fechada e orientável de dimensão 3 , com alguns invariantes topológicos. Mais especificamente, como $W_{g}$ é um poliedro e $\bar{g}: W_{g} \rightarrow \mathbb{R}^{2}$ uma aplicação simplicial, aplicamos a fórmula demostrada por Ballesteros \& Saeki $[\mathrm{BS}]$, em $\bar{g}$. Assim, utilizando a definição de índice $\operatorname{Ind}_{f}(v)$ dada em [BS], deduzimos uma fórmula em $\mathbb{Z}$ que nos permite, por exemplo, concluir que as cúspides de $g$ ocorrem aos pares. Este é um resultado similar àquele obtido por Izumiya \& Marar em [IM1], [IM2] e [IM3]. Por outro lado, damos uma nova definição de índice, não relacionada à fatorização de Stein, e com isso obtemos uma segunda fórmula que nos possibilita uma nova demonstração para o seguinte resultado: "A característica de Euler de toda variedade fechada de dimensão três é zero", sem utilizar a dualidade de Poincaré. Além do valor intrínseco deste já bem conhecido resultado e de sua nova demonstração, o incluímos neste capítulo por ter sido obtido por métodos combinatórios.

No Capítulo 6, analogamente à teoria desenvolvida no Capítulo 5, estudamos aplicações $g: N^{4} \rightarrow \mathbb{R}^{3}$, onde $g$ é uma aplicação $\mathcal{C}^{\infty}$-estável e $N^{4}$ é uma variedade 4-dimensional fechada e orientável. Novamente, obtemos duas fórmulas relacionando as singularidades da aplicação com invariantes topológicos. Ambas são fórmulas relacionando grandezas em $\mathbb{Z}$; a partir dela obtemos outras $(\bmod 2)$ que possibilitam, por exemplo, tirar conclusões sobre a paridade das singularidades de $g$. Como corolário obtemos outros resultados interessantes, um dos quais nos possibilitou detectar um pequeno erro em um dos exemplos apresentado por Kobayashi em [Ko]. 


\section{Capítulo 1}

\section{A fatorização de Stein e sua triangulação}

As ferramentas básicas deste trabalho são complexos e aplicações simpliciais. Outro conceito fundamental é o da fatorização de Stein de aplicações contínuas. Enunciamos, neste capítulo, conceitos básicos de complexos simpliciais geométricos e abstratos, bem como definições e propriedades de aplicações simpliciais. Definimos, também, a fatorização de Stein para uma aplicação contínua e a sua triangulação, e enunciamos um dos resultados principais deste trabalho (Teorema 1.3.8), onde apresentamos condições suficientes para que a fatorização de Stein de uma aplicação contínua seja triangulável. Na última seção, utilizando um resultado de Verona [Ve1], [Ve2], concluímos que a fatorização de Stein de toda aplicação diferenciável, topologicamente estável e própria, também é triangulável.

\subsection{Complexo simplicial geométrico}

Nesta seção concentramos todas as definições e resultados referentes a complexos simpliciais necessários para o desenvolvimento do nosso trabalho. Eles foram extraídos basicamente de Rourke \& Sanderson [RS], Munkres [Mu] e 
Hudson $[\mathrm{Hu}]$.

Iniciamos com a definição de "join" de dois subconjuntos do $\mathbb{R}^{n}$, o qual será necessário na definição de um simplexo do $\mathbb{R}^{n}$. Propriedades referentes ao "join" serão vistos na Seção 2.1 .

Definição 1.1.1 Sejam $P, Q \subset \mathbb{R}^{n}$ subconjuntos. O "join" $P Q$ é o subconjunto $P Q=\{\lambda p+\mu q\}$, onde $p \in P, q \in Q, \lambda, \mu \in \mathbb{R}, \lambda, \mu \geq 0 \mathrm{e}$ $\lambda+\mu=1$. Então, $P Q$ coincide com a união de todos os segmentos com uma. extremidade em $P$ e outra em $Q$. Se $P=\emptyset, P Q=Q$.

Definição 1.1.2 Dois conjuntos $P, Q \subset \mathbb{R}^{n}$ são ditos independentes se cada ponto do "join" $P Q$ pode ser escrito unicamente na forma $\lambda p+\mu q$, com $\lambda \geq 0, \mu \geq 0, \lambda+\mu=1, p \in P$ e $q \in Q$. Equivalentemente, $P \cap Q=\emptyset$ e os interiores dos arcos $p_{1} q_{1}$ e $p_{2} q_{2}$ são disjuntos a menos que $p_{1}=p_{2}$ e $q_{1}=q_{2}$, onde $p_{1}, p_{2} \in P$ e $q_{1}, q_{2} \in Q$. Também definimos $\emptyset$ e qualquer subconjunto $P \subset \mathbb{R}^{n}$ como sendo independentes.

Definição 1.1.3 Um conjunto finito $\left\{v_{0}, v_{1}, \ldots, v_{n}\right\} \subset \mathbb{R}^{m}$, com $n \leq m$, é dito independente se ele não está contido em nenhum subespaço afim de dimensão menor que $n$ ou, equivalentemente, se os vetores $\left\{v_{i}-v_{0}\right\}, 1 \leq i \leq$ $n$, são linearmente independentes.

Definição 1.1.4 Um simplexo de dimensão $n$, ou simplesmente, um n-simplexo $\sigma \subset \mathbb{R}^{m}$ é formado pelos repetidos "joins" $v_{0} v_{1} \cdots v_{n}$ de $n+1$ pontos independentes. Denotamos por $\operatorname{dim} \sigma=n$. Os pontos $v_{i}$ são ditos vértices de $\sigma$ e dizemos que eles geram o simplexo $\sigma$. Um simplexo $\tau$ gerado por um subconjunto dos vértices $\left\{v_{0}, v_{1}, \ldots, v_{n}\right\}$ é chamado face de $\sigma$ e denotado por $\tau<\sigma$. Se $\tau \neq \sigma$ dizemos que $\tau$ é uma face própria de $\sigma$ e o denotamos por $\tau \ll \sigma$. Os vértices e o conjunto vazio também são faces. 
Definimos, ainda, a fronteira de $\sigma$, denotada por $\partial \sigma$, como sendo a união de todas as faces próprias de $\sigma$. O interior de $\sigma$, denotado por $\stackrel{\circ}{\sigma}$, é definido por $\stackrel{\circ}{\sigma}=\sigma \backslash \partial \sigma$.

Todo ponto $x \in \sigma=v_{0} v_{1} \cdots v_{n}$ se escreve de forma única como uma soma $\sum_{i=0}^{n} \alpha_{i} v_{i}$, onde $\alpha_{i} \geq 0$ e $\sum_{i=0}^{n} \alpha_{i}=1$ e, neste caso, chamamos $\alpha_{0}, \ldots, \alpha_{n}$ de coordenadas baricêntricas de $x$. O ponto $b_{\sigma}=\sum_{i=0}^{n} \frac{1}{n+1} v_{i}$ é denominado baricentro de $\sigma$. Não faremos distinção entre o 0 -simplexo $\langle v\rangle$ e o vértice $v$.

Usamos a seguinte notação $\sigma=\left\langle v_{0} v_{1} \cdots v_{n}\right\rangle$.

Definição 1.1.5 Um complexo simplicial geométrico ou, simplesmente, complexo simplicial $K$ é uma coleção de simplexos em algum $\mathbb{R}^{n}$ satisfazendo:

(1) se $\sigma \in K$ e $\tau<\sigma$, então $\tau \in K$, e

(2) se $\tau, \sigma \in K$, então $\tau \cap \sigma$ é uma face comum a $\tau$ e a $\sigma$.

Definimos, ainda, $K^{(0)}$ como sendo o conjunto de todos os 0 -simplexos (vértices) de $K$.

Dizemos que um complexo simplicial $K$ é localmente finito se, para cada vértice $v \in K^{(0)}$, existe apenas um número finito de simplexos em $K$ que têm $v$ como um de seus vértices.

Observação 1.1.6 Dado um simplexo $\sigma$, a coleção de todas as suas faces satisfaz a definição de complexo simplicial. Denotamos este complexo simplicial também por $\sigma$.

Definição 1.1.7 Dado um complexo simplicial geométrico $K$, seja $|K|$ a união de todos os simplexos de $K$. Consideremos em cada $\sigma \in K$ a topologia induzida do $\mathbb{R}^{n}$ e em $|K|$ a seguinte topologia: um subconjunto $A \subset|K|$ é fechado em $|K|$ se, e somente se, $A \cap \sigma$ é um fechado em $\sigma$, para cada $\sigma \in K$. Se $K$ é um complexo simplicial finito, então a topologia de $|K|$ coincide com 
a topologia induzida de $\mathbb{R}^{n}$ [Mu,pg.9]. O espaço topológico $|K|$ é chamado espaço subjacente de $K$. Note que $|K|$ é sempre Hausdorff [Mu, Lemma 2.4, pg.10]. Um espaço topológico que é o espaço subjacente de algum complexo simplicial é chamado poliedro.

Definição 1.1.8 Dados simplexos $\sigma_{1}, \ldots, \sigma_{r}$ tais que $\sigma_{i} \cap \sigma_{j}$ é uma face comum a $\sigma_{i}$ e a $\sigma_{j}$, para quaisquer $1 \leq i<j \leq r$, dizemos que um complexo simplicial geométrico $K$ é gerado pelos simplexos $\sigma_{1}, \ldots, \sigma_{r}$ se $K$ for a coleção de todas as faces de $\sigma_{1}, \ldots, \sigma_{r}$.

Definição 1.1.9 Sejam $K$ e $L$ dois complexos simpliciais. Dizemos que $f: K \rightarrow L$ é uma aplicação simplicial se:

a) $v$ é um vértice de $K \Rightarrow f(v)$ é um vértice de $L$; e

b) $\sigma=\left\langle v_{0} v_{1} \cdots v_{n}\right\rangle$ é um simplexo de $K \Rightarrow f(\sigma)=\left\langle f\left(v_{0}\right) f\left(v_{1}\right) \cdots f\left(v_{n}\right)\right\rangle$ é um simplexo de $L$ (pode haver repetições entre $f\left(v_{0}\right), f\left(v_{1}\right), \ldots, f\left(v_{n}\right)$, ou seja, a dimensão de $f(\sigma)$ pode ser menor ou igual a $n)$.

Dizemos, ainda, que $f$ é uma aplicação nâo-degenerada se $\operatorname{dim}(f(\sigma))=$ $\operatorname{dim} \sigma$, para qualquer $\sigma \in K$.

Observação 1.1.10 Seja $f: K \rightarrow L$ uma aplicação simplicial. Dado um ponto $x \in|K|$, existe um único simplexo $\sigma=\left\langle v_{0} v_{1} \cdots v_{n}\right\rangle \in K$ tal que $x \in \stackrel{\circ}{\sigma}$, e existem números reais positivos $\alpha_{0}, \ldots, \alpha_{n}$, unicamente determinados, tais que $\sum_{i=0}^{n} \alpha_{i}=1$ e $x$ se escreve como $x=\sum_{i=0}^{n} \alpha_{i} v_{i}$. Definimos a aplicação $|f|:|K| \rightarrow|L|$ da seguinte forma:

$$
|f|(x)=|f|\left(\sum_{i=0}^{n} \alpha_{i} v_{i}\right)=\sum_{i=0}^{n} \alpha_{i} f\left(v_{i}\right) \in(\stackrel{\circ}{f(\sigma)}) .
$$


Definição 1.1.11 Seja $f: K \rightarrow L$ uma aplicação simplicial entre os complexos simpliciais $K$ e $L$. Denotamos por $f^{(0)}: K^{(0)} \rightarrow L^{(0)}$ a restrição de $f$ ao conjunto $K^{(0)}$.

Definição 1.1.12 A aplicação $|f|:|K| \rightarrow|L|$, definida acima, é contínua [Mu, Lemma 2.7, pg.12]. Se $f^{(0)}: K^{(0)} \rightarrow L^{(0)}$ for uma bijeção, então $|f|:|K| \rightarrow|L|$ é um homeomorfismo [Mu, Lemma 2.8, pg.12] e, neste caso, dizemos que $|f|$ é um homeomorfismo simplicial, ou que $f$ é um isomorfismo entre $K$ e $L$.

Lema 1.1.13 Seja $X \subset|K|$ um subespaço. Se $K$ é localmente finito, então um subconjunto $A \subset X$ é fechado de $X$ se, e somente se, $A \cap \sigma$ é fechado de $X \cap \sigma, \operatorname{para} \forall \sigma \in K$.

\section{Demonstração:}

Se $A \subset X$ é fechado, então claramente $A \cap \sigma$ é fechado de $X \cap \sigma$ para $\forall \sigma \in K$. Reciprocamente, vamos assumir que $A \cap \sigma$ é fechado de $X \cap \sigma$ para $\forall \sigma \in K$. Neste caso, temos

$$
\begin{aligned}
A \cap X & =\left(\bigcup_{\sigma \in K} A \cap \sigma\right) \cap X \\
& =\bigcup_{\sigma \in K}(A \cap \sigma \cap X) \\
& =\bigcup_{\sigma \in K}(\overline{A \cap \sigma} \cap X) \\
& =\left(\bigcup_{\sigma \in K} \overline{A \cap \sigma}\right) \cap X \\
& \stackrel{(*)}{=} \bar{A} \cap X,
\end{aligned}
$$

onde a igualdade (*) vale porque $K$ é localmente finito. Por isso $A$ é fechado de $X$. Isto completa a demonstração. 
Lema 1.1.14 Sejam $K$ um complexo simplicial, $X \subset|K|$ um subespaço $e$ $Y$ um espaço topológico. Suponhamos uma das seguintes condições:

(1) $X$ é fechado de $|K|$, ou

(2) $X$ é aberto de $|K|$, ou

(3) $K$ é localmente finito.

Então uma aplicação $h: X \rightarrow Y$ é contínua se, e somente se, $\left.h\right|_{X \cap \sigma}: X \cap \sigma \rightarrow$ $Y$ é contínua para todo $\sigma \in K$.

\section{Demonstração:}

Vamos primeiro assumir que $X$ é fechado. Então, temos

$$
\begin{aligned}
& h \text { é contínua } \\
\Longleftrightarrow & h^{-1}(F) \text { é fechado de } X \text { para qualquer fechado } F \text { de } Y \\
\Longleftrightarrow & h^{-1}(F) \text { é fechado de }|K| \text { para qualquer fechado } F \text { de } Y \\
\Longleftrightarrow & h^{-1}(F) \cap \sigma \text { é fechado de } \sigma \text { para qualquer fechado } F \\
& \text { de } Y \text { e para } \forall \sigma \in K \\
\Longleftrightarrow & h^{-1}(F) \cap \sigma \text { é fechado de } \sigma \cap X \text { para qualquer fechado } F \\
& \text { de } Y \text { e para } \forall \sigma \in K \\
\Longleftrightarrow & \left(\left.h\right|_{\sigma \cap X}\right)^{-1}(F) \text { é fechado de } \sigma \cap X \text { para qualquer fechado } F \\
& \text { de } Y \text { e para } \forall \sigma \in K \\
\Longleftrightarrow & \left.h\right|_{\sigma \cap X} \text { é contínua para } \forall \sigma \in K .
\end{aligned}
$$

No caso de $X$ ser aberto, podemos usar conjuntos abertos no lugar de $F$ acima. Quando $K$ é localmente finito, podemos usar um argumento similar junto com o Lema 1.1.13. Isto completa a demonstração. 
Observação 1.1.15 Na Definição 1.1.5 de um complexo simplicial, todos os simplexos têm que estar contidos em $\mathbb{R}^{n}$, para algum $n$; isto coloca uma limitação sobre a cardinalidade de $K$ e sobre a dimensão dos simplexos. Contornamos esta situação da seguinte forma: sejam $J$ um conjunto de índices arbitrários e $\mathbb{R}^{J}$ o conjunto de todas as funções de $J$ em $\mathbb{R}$. Denotamos um elemento de $\mathbb{R}^{J}$ por $\left(x_{\alpha}\right)_{\alpha \in J}$. Consideremos em $\mathbb{R}^{J}$ a adição e a multiplicação por escalar coordenada-a-coordenada. Agora, denotemos por $E^{J}$ o subconjunto de $\mathbb{R}^{J}$ de todos os pontos $\left(x_{\alpha}\right)_{\alpha \in J}$, tais que $x_{\alpha} \neq 0$ apenas para um número finito de índices $\alpha \in J$. Ao conjunto $E^{J}$ munido da topologia induzida pela métrica $|x-y|=\max \left\{\left|x_{\alpha}-y_{\alpha}\right|\right\}_{\alpha \in J}$ chamamos de espaço Euclideano generalizado. Todas as definições anteriores para complexos em $\mathbb{R}^{n}$ se generalizam para complexos em $E^{J}$. O espaço $E^{J}$ pode ser visto como uma união de "cópias" de $\mathbb{R}^{n}$, bastando para isso considerar a aplicação $\left(a_{1}, \ldots, a_{n}\right) \mapsto\left(a_{1}, \ldots, a_{n}, 0, \ldots\right)$; assim, para uma coleção finita de simplexos em $E^{J}$, podemos considerar uma "cópia" deste em $\mathbb{R}^{n}$, para algum $n$ [Mu, pg.13]. Portanto, a partir de agora vamos considerar os complexos simpliciais e simplexos em $E^{J}$.

Definição 1.1.16 Seja $K$ um complexo simplicial em $E^{J}$. Um complexo simplicial $K^{\prime}$ é dito uma subdivisão de $K$ se:

(1) cada simplexo de $K^{\prime}$ está contido em um simplexo de $K$;

(2) cada simplexo de $K$ é uma união finita de simplexos de $K^{\prime}$. Neste caso, $|K|=\left|K^{\prime}\right|$.

Observação 1.1.17 A definição de subdivisão baricêntrica de um complexo simplicial se encontra em [Mu, pg.85].

Lema 1.1.18 Se um complexo simplicial $\tilde{K}$ é localmente finito, então qualquer subdivisão $K$ de $\tilde{K}$ também é localmente finito. 
A demonstração do Lema acima é imediata a partir da definição.

Proposição 1.1.19 Seja $\tilde{f}: \tilde{K} \rightarrow \tilde{L}$ uma aplicação simplicial entre dois complexos simpliciais localmente finitos. Dada uma subdivisẫo $L$ de $\tilde{L}$, existem uma subdivisão $K$ de $\tilde{K}$ e uma aplicação simplicial $f: K \rightarrow L$ tal que $|f|=|\tilde{f}|$, onde $|f|:|K| \rightarrow|L| e|\tilde{f}|:|\tilde{K}| \rightarrow|\tilde{L}|$.

Demonstração: [Hu, pg.84].

\subsection{Complexo simplicial abstrato}

O conceito de complexo simplicial abstrato é pouco utilizado nos textos e cursos básicos de topologia combinatória, porém ele é essencial para demonstrarmos um dos resultados principais deste trabalho, Teorema 1.3.8, que está enunciado na Seção 1.3. Além da definição de complexos simpliciais abstratos enunciamos um resultado que relaciona-os com os complexos simpliciais geométricos.

Definição 1.2.1 Um complexo simplicial abstrato é uma coleção $\mathcal{K}$ de conjuntos finitos, não vazios, tal que, se $\sigma$ é um elemento de $\mathcal{K}$, então todo subconjunto não vazio de $\sigma$ também é um elemento de $\mathcal{K}$. Um elemento $\sigma$ de $\mathcal{K}$ é chamado um simplexo de $\mathcal{K}$ e, se $\sigma$ é um conjunto finito com $n+1$ elementos, dizemos que $\sigma$ tem dimensão $n$ e escrevemos $\operatorname{dim} \sigma=n$. Todo subconjunto não vazio $\tau$ de $\sigma$ é chamado face de $\sigma$ e, se $\tau \neq \sigma$, dizemos que $\tau$ é uma face própria de $\sigma$ e denotamos por $\tau \ll \sigma$. A dimensão de $\mathcal{K}$ é a dimensão máxima de seus simplexos, ou é infinito se não existir um simplexo de dimensão máxima. Os conjuntos unitários de $\mathcal{K}$ são denominados vértices de $\mathcal{K}$. O conjunto dos vértices de $\mathcal{K}$ é denotado por $\mathcal{K}^{(0)}$. Não faremos distinção entre os vértices $v$ de $\mathcal{K}^{(0)}$ e os 0 -simplexos $\{v\}$ de $\mathcal{K}$. Uma subcoleção de $\mathcal{K}$ que por si só é um complexo é denominado um subcomplexo de $\mathcal{K}$. 
Dizemos que dois complexos simpliciais abstratos $\mathcal{K}$ e $\mathcal{L}$ são isomorfos se existir uma bijeção $f$ entre os vértices de $\mathcal{K}$ e os vértices de $\mathcal{L}$ tal que, $\left\{v_{0}, \ldots, v_{n}\right\} \in \mathcal{K}$ se, e somente se, $\left\{f\left(v_{0}\right), \ldots, f\left(v_{n}\right)\right\} \in \mathcal{L}$.

Observação 1.2.2 No decorrer de toda a tese, chamaremos $\mathcal{K}$ de complexo simplicial abstrato, para distingüí-lo do complexo simplicial geométrico $K$.

Definição 1.2.3 Seja $K^{(0)}$ o conjunto dos vértices de um complexo simplicial geométrico $K$. A coleção de todos os subconjuntos $\left\{v_{0}, \ldots, v_{n}\right\}$ de $K^{(0)}$ tais que os vértices $v_{0}, \ldots, v_{n}$ geram um simplexo de $K$ é chamada esquema de vértices de $K$. Notemos que o conjunto assim definido é um complexo simplicial abstrato.

Teorema 1.2.4 (1) Todo complexo simplicial abstrato $\mathcal{K}$ é isomorfo ao esquema de vértices de algum complexo simplicial geométrico $K$.

(2) Dois complexos simpliciais geométricos são isomorfos se, e somente se, seus esquemas de vértices são isomorfos como complexos simpliciais abstratos.

Demonstração: [Mu,pg.15].

Definição 1.2.5 Se o complexo simplicial abstrato $\mathcal{K}$ é isomorfo a um esquema de vértices de um complexo simplicial geométrico $K$, chamamos $K$ de realização geométrica de $\mathcal{K}$. Ele é unicamente determinado, a menos de um isomorfismo.

Observação 1.2.6 Na Definição 1.2.3, $K$ é uma realização geométrica do seu esquema de vértices.

Definição 1.2.7 Dado $\mathcal{K}$ um complexo simplicial abstrato; construímos uma subdivisão baricêntrica $\operatorname{Sd}(\mathcal{K})$ da seguinte forma: o conjunto dos vértices de 
$\operatorname{Sd}(\mathcal{K})$ é dado por $\operatorname{Sd}(\mathcal{K})^{(0)}=\{\sigma: \sigma \in \mathcal{K}\}$. Os simplexos de $\operatorname{Sd}(\mathcal{K})$ são os conjuntos $\left\{\sigma_{0}, \sigma_{1}, \ldots, \sigma_{q}\right\}$, onde $\sigma_{0} \ll \sigma_{1} \ll \cdots \ll \sigma_{q}$. O conjunto $\operatorname{Sd}(\mathcal{K})$ assim definido é um complexo simplicial abstrato. Se $K$ é uma realização geométrica de $\mathcal{K}$, então a subdivisão baricêntrica de $K$ (Observação 1.1.17) é uma realização geométrica de $\operatorname{Sd}(\mathcal{K})$.

Definição 1.2.8 Uma aplicação simplicial $\mathfrak{f}: \mathcal{K} \rightarrow \mathcal{L}$ entre os complexos simpliciais abstratos $\mathcal{K}$ e $\mathcal{L}$, é uma aplicação $\mathfrak{f}: \mathcal{K}^{(0)} \rightarrow \mathcal{L}^{(0)}$ tal que, quando $\left\{v_{0}, \ldots, v_{q}\right\}$ é um simplexo em $\mathcal{K}$, então a sua imagem $\left\{\varphi\left(v_{0}\right), \ldots, \varphi\left(v_{q}\right)\right\}$ é sempre um simplexo em $\mathcal{L}$ (a segunda lista de vértices pode ter repetições).

Definição 1.2.9 Dizemos que uma aplicação simplicial $f: \mathcal{K} \rightarrow \mathcal{L}$ entre os complexos simpliciais abstratos $\mathcal{K}$ e $\mathcal{L}$ é não-degenerada se $\operatorname{dim}(\mathfrak{f}(\sigma))=\operatorname{dim} \sigma$, para qualquer $\sigma \in \mathcal{K}$.

Observação 1.2.10 Dada uma aplicação simplicial não-degenerada $f: K \rightarrow$ $L$, a aplicação $f: \mathcal{K} \rightarrow \mathcal{L}$, entre os esquemas de vértices de $K$ e $L$, é também uma aplicação simplicial não-degenerada.

Observação 1.2.11 A partir deste ponto, "simplexo" (respectivamente, "complexo simplicial") significará simplexo (respectivamente, complexo simplicial) geométrico.

\subsection{A fatorização de Stein e sua triangulação}

O conceito de fatorização de Stein foi introduzido por Burlet \& de Rham $[\mathrm{BdR}]$; depois disso vários autores trabalharam com este conceito; podemos citar [PF], [Sa1], [MPS], dentre outros. Ela é uma ferramenta importante na teoria global de singularidades de aplicações diferenciáveis entre variedades, $g: M^{n} \rightarrow \mathbb{R}^{p}$. Por exemplo, usando essencialmente a fatorização de Stein, 
alguns autores, como Burlet \& de Rham [BdR], no caso $n=3$ e $p=2$, e Saeki [Sa1], no caso $n \geq p$, conseguiram caracterizar as variedades fechadas que admitem aplicações genéricas especiais.

Definição 1.3.1 Seja $g: N \rightarrow P$ uma aplicação contínua entre dois espaços topológicos $N$ e $P$. Dizemos que dois pontos $x_{1}, x_{2} \in N$ são equivalentes se $g\left(x_{1}\right)=g\left(x_{2}\right)$ e $x_{1}, x_{2}$ pertencem à mesma componente conexa de $g^{-1}\left(g\left(x_{1}\right)\right)=g^{-1}\left(g\left(x_{2}\right)\right)$. Denotamos por $W_{g}$ o espaço quociente de $N$ pela relação de equivalência descrita acima, munido com a topologia quociente, e $q_{g}: N \rightarrow W_{g}$ a aplicação quociente. Então existe uma única $\bar{g}: W_{g} \rightarrow P$ contínua tal que $g=\bar{g} \circ q_{g}$. O espaço quociente $W_{g}$ ou o diagrama comutativo

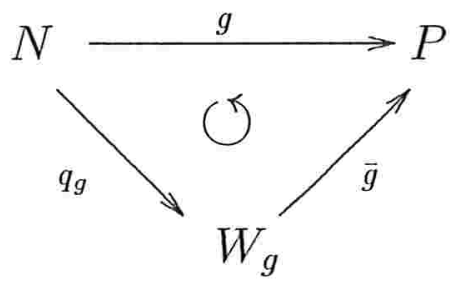

são chamados fatorização de Stein de $g$.

Observação 1.3.2 A relação definida acima é uma relação de equivalência.

Intuitivamente, o espaço quociente coincide com o espaço das componentes conexas das fibras da aplicação $g$. Por isso, cada fibra da aplicação quociente $q_{g}$ é conexa.

Exemplo 1.3.3 Seja $g: T^{2} \rightarrow I$ a aplicação altura do toro $T^{2}$, onde $I$ é um intervalo. Então a fatorização de Stein é dada como na figura abaixo. 


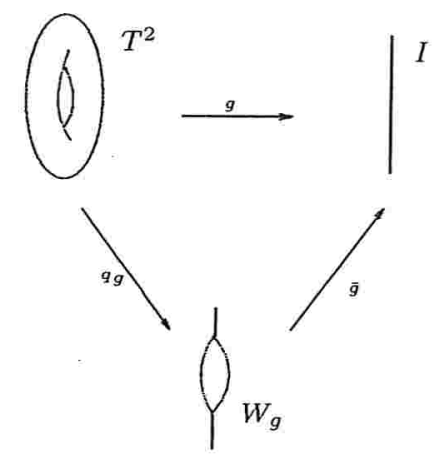

Observação 1.3.4 Como pode ser verificado no Exemplo 1.3.3, a fatorização de Stein $W_{g}$ pode não ser uma variedade, mesmo que $N$ e $P$ o sejam.

Definimos a seguir triangulação de aplicações contínuas.

Definição 1.3.5 Dada uma aplicação contínua $g: N \rightarrow P$ entre dois espaços topológicos, dizemos que $g$ é triangulável se existirem complexos simpliciais $K, L$, uma aplicação simplicial $f: K \rightarrow L$ e dois homeomorfismos $\lambda:|K| \rightarrow$ $N$ e $\mu:|L| \rightarrow P$ que fazem o seguinte diagrama comutar:

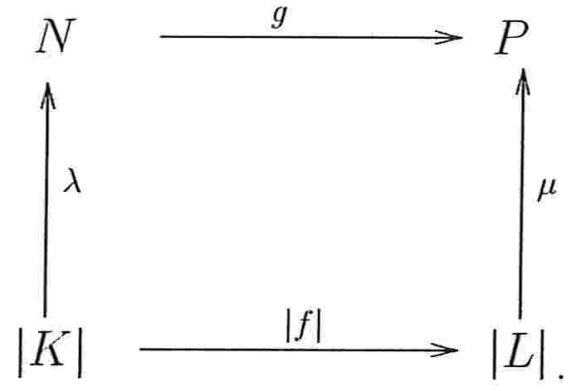

Neste caso, chamamos $\lambda, \mu$ e $f$ de triangulações de $N, P$ e $g$, respectivamente.

Observação 1.3.6 Para simplificar a notação também chamamos $K$ e $L$ de triangulações de $N$ e $P$, respectivamente. 
Definição 1.3.7 Seja $g: N \rightarrow P$ uma aplicação contínua entre espaços topológicos. Uma triangulação da fatorização de Stein de $g$ consiste dos seguintes objetos: complexos simpliciais $K, L$ e $V$; aplicações simpliciais $f$ : $K \rightarrow L, \varphi: K \rightarrow V$ e $\psi: V \rightarrow L$; homeomorfismos $\lambda:|K| \rightarrow N, \mu:|L| \rightarrow$ $P, \Theta:|V| \rightarrow W_{g}$ tais que o diagrama

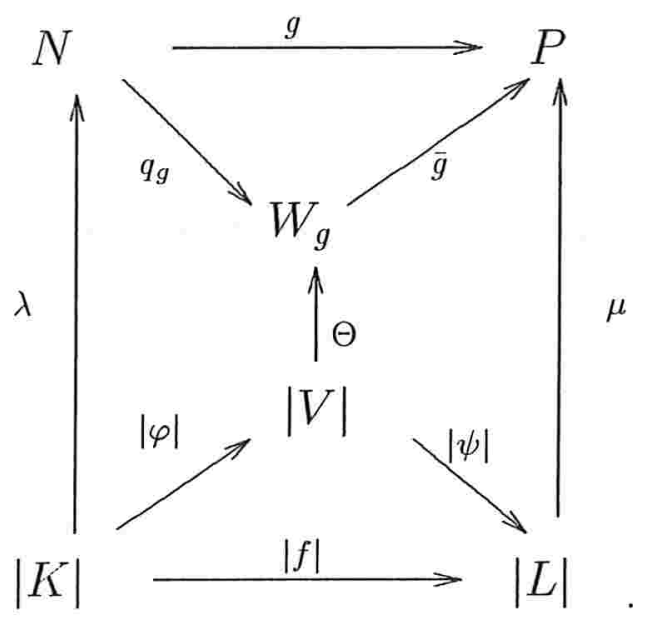

é comutativo. Caso tais objetos existam, dizemos que $W_{g}$, a fatorização de Stein de $g$, é triangulável e chamamos $\varphi, \psi$ e $V$ de triangulações de $q_{g}, \bar{g}$ e $W_{g}$, respectivamente.

O resultado enunciado abaixo (Teorema 1.3.8) é um dos mais importantes deste trabalho. Ele nos diz que a fatorização de Stein, de uma certa classe de aplicações, é um poliedro. Esta propriedade nos possibilita a utilização da topologia combinatória para concluir resultados interessantes a respeito dos domínios de aplicações trianguláveis, bem como do seu conjunto singular. Utilizando o Teorema 1.3.8, no Capítulo 4 deduzimos uma expressão mais detalhada da fórmula envolvendo característica de Euler, demonstrada por Ballesteros \& Saeki [BS]; no Capítulo 5 demonstramos uma fórmula que associa o número de singularidades de uma aplicação $\mathcal{C}^{\infty}$-estável $M^{3} \rightarrow \mathbb{R}^{2} \mathrm{com}$ 
certos invariantes topológicos e, no Capítulo 6, encontramos outra fórmula para aplicações $\mathcal{C}^{\infty}$-estáveis $M^{4} \rightarrow \mathbb{R}^{3}$.

Teorema 1.3.8 Seja $g: N \rightarrow P$ uma aplicação contínua entre espaços topológicos. Suponhamos que $N$ e P são localmente compactos e g é própria. Se $g$ é triangulável, então a fatorização de Stein de g também é triangulável.

Este teorema será demonstrado na Seção 3.3.

\subsection{Aplicações $\mathcal{C}^{\infty}$ trianguláveis}

Nesta seção definimos aplicações topologicamente estáveis e $\mathcal{C}^{\infty}$-estáveis, e relacionamos alguns resultados importantes como o de Verona [Ve1], [Ve2] que apresenta uma classe de aplicações diferenciáveis que são trianguláveis.

Seja $g: N \rightarrow P$ uma aplicação diferenciável $\left(\mathcal{C}^{\infty}\right)$ entre duas variedades diferenciáveis. Denotamos por $S(g)$ o conjunto dos pontos singulares de $g$, isto é, $S(g)=\left\{x \in N: \operatorname{rank} d g_{x}<\min \{\operatorname{dim} N, \operatorname{dim} P\}\right\}$, o qual é chamado de conjunto singular de $g$. Esta notação será usada nos Capítulos 5 e 6 .

Definição 1.4.1 Sejam $N$ e $P$ duas variedades diferenciáveis $\mathcal{C}^{\infty}$ sem bordo e consideremos o espaço $\mathcal{C}^{\infty}(N, P)$ das aplicações diferenciáveis $N \rightarrow P$ com a topologia de Whitney [GG, pg.42]. Uma aplicação diferenciável $g$ : $N \rightarrow P$ é topologicamente estável (respectivamente, $\mathcal{C}^{\infty}$-estável) se existir uma vizinhança $U$ de $g$ em $\mathcal{C}^{\infty}(N, P)$ tal que, para qualquer $f \in U$, existem homeomorfismos (respectivamente, difeomorfismos) $\varphi: N \rightarrow N$ e $\psi: P \rightarrow P$ que fazem o seguinte diagrama comutar: 


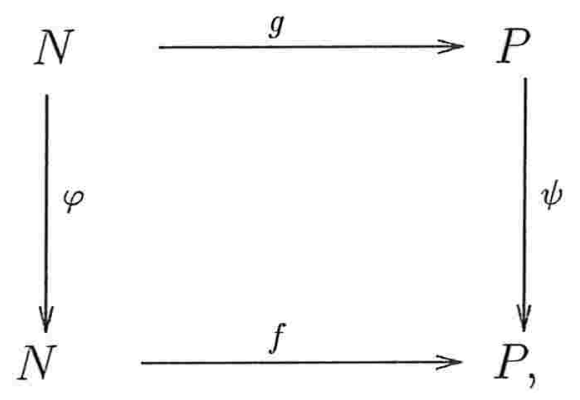

isto é, $f=\psi \circ g \circ \varphi^{-1}$.

Exemplo 1.4.2 Os exemplos clássicos de aplicações estáveis são: submersões, funções de Morse e imersões com cruzamentos normais [GG]. Outros exemplos de aplicações estáveis podem ser vistos nos Capítulos 5 e 6 .

Teorema 1.4.3 [Ma] O conjunto das aplicações topologicamente estáveis é denso dentro do conjunto $\mathcal{C}_{p r}^{\infty}(N, P)$ das aplicações diferenciáveis e próprias entre duas variedades diferenciáveis $N$ e $P$.

O teorema a seguir é fundamental para o desenvolvimento dos Capítulos 3, 4,5 e 6 .

Teorema 1.4.4 [Ve1], [Ve2] Toda aplicação topologicamente estável e própria entre duas variedades diferenciáveis é triangulável.

Corolário 1.4.5 [Ve1], [Ve2] O conjunto das aplicações próprias entre variedades diferenciáveis e trianguláveis de $\mathcal{C}^{\infty}(N, P)$ contém um conjunto aberto e denso de $\mathcal{C}_{p r}^{\infty}(N, P)$.

Observação 1.4.6 Shiota mostra em [Sh] que as aplicações de Thom-Mather, cuja classe é maior do que a classe das aplicações topologicamente estáveis, também são trianguláveis. Portanto, pelo Teorema 1.3.8, temos que a fatorização de Stein destas aplicações também são trianguláveis. 
Corolário 1.4.7 Seja $g: N \rightarrow P$ uma aplicação diferenciável e própria entre variedades diferenciáveis. Se g é topologicamente estável, ou se g é uma aplicação de Thom-Mather, então a fatorização de Stein de g é triangulável.

Corolário 1.4.8 O conjunto das aplicações próprias de $\mathcal{C}^{\infty}(N, P)$ cujas fatorizações de Stein são trianguláveis contém um conjunto aberto e denso de $\mathcal{C}_{p r}^{\infty}(N, P)$. 


\section{Capítulo 2}

\section{Um estudo sobre aplicações simpliciais}

Neste capítulo, estudamos as fibras $|f|^{-1}(y)$ da aplicação $|f|:|K| \rightarrow|L|$, onde $f: K \rightarrow L$ é uma aplicação simplicial e $y \in|L|$. Tal estudo é necessário para mostrarmos que a fatorização de Stein $W_{|f|}$ (Definição 1.3.1) é um poliedro. De fato, provamos que se $\sigma \in f(K)$ é um simplexo tendo $b_{\sigma}$ como baricentro, então $\mid f \|_{|f|^{-1}(\stackrel{\circ}{\sigma})}$ é um fibrado trivial com fibra $|f|^{-1}\left(b_{\sigma}\right)$.

Alguns dos resultados, como o citado acima, podem ser encontrados, por exemplo, em [MS, §20], mas por tratarem-se de ferramentas essenciais para o desenvolvimento da tese, optamos por apresentá-los novamente aqui.

Por comodidade, neste capítulo, não faremos distinção entre as notações de complexos simpliciais geométricos e de seus espaços subjacentes, ou seja, usaremos a notação $f: K \rightarrow L$ no lugar de $|f|:|K| \rightarrow|L|$. No Capítulo 3 voltaremos a utilizar a notação $|K|$ para o espaço subjacente do complexo simplicial $K$. 


\subsection{O "join" entre subconjuntos do $\mathbb{R}^{n}$}

Como foi visto no Capítulo 1 , definimos um $n$-simplexo através do "join" de subconjuntos do $\mathbb{R}^{n}$. Relacionamos abaixo alguns resultados sobre "joins" necessários para estudar o comportamentos das fibras de $|f|$, onde $f$ é uma aplicação simplicial.

Proposição 2.1.1 Sejam $P, Q$ subconjuntos compactos e independentes do $\mathbb{R}^{n}$. Então o "join" $P Q$ é homeomorfo a $P * Q$, onde

$$
P * Q=(P \times[0,1] \times Q) / \sim
$$

definido pela seguinte relação de equivalência:

$$
(p, t, q) \sim\left(p^{\prime}, t^{\prime}, q^{\prime}\right) \Leftrightarrow\left\{\begin{array}{l}
(p, t, q)=\left(p^{\prime}, t^{\prime}, q^{\prime}\right) \\
\text { ou } \\
t=t^{\prime}=0 \text { e } p=p^{\prime} \\
\text { ou } \\
t=t^{\prime}=1 \text { e } q=q^{\prime} .
\end{array}\right.
$$

Demonstração:

Consideremos o seguinte diagrama comutativo:

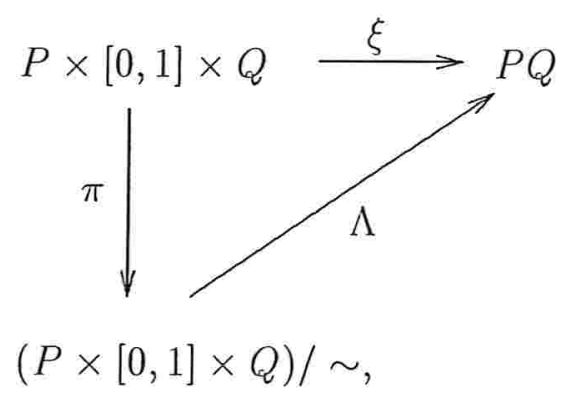

onde $\pi$ é a projeção que associa a cada $(p, t, q) \in P \times[0,1] \times Q$ a classe de equivalência $[p, t, q]$ definida pela relação de equivalência acima, $\xi$ é a aplicação que a cada terna ordenada $(p, t, q) \in P \times[0,1] \times Q$ associa o 
elemento $(1-t) p+t q$ de $P Q$ e $\Lambda$ é a aplicação que associa a cada classe de equivalência $[p, t, q] \in P * Q$ o elemento $(1-t) p+t q$ de $P Q$.

Primeiro observemos que $\Lambda$ está bem definida, pois considerando dois representantes diferentes $(p, t, q)$ e $\left(p^{\prime}, t^{\prime}, q^{\prime}\right)$ de uma mesma classe de equivalência em $P * Q$, temos que:

1) se $t \in(0,1)$, então, pela definição, temos que $(p, t, q)=\left(p^{\prime}, t^{\prime}, q^{\prime}\right)$ e isto implica que $\Lambda([p, t, q])=\Lambda\left(\left[p^{\prime}, t^{\prime}, q^{\prime}\right]\right)$;

2) se $t=0$, então $t^{\prime}=0, p=p^{\prime}$ e isto implica que $\Lambda([p, 0, q])=p=p^{\prime}=$ $\Lambda\left(\left[p^{\prime}, 0, q^{\prime}\right]\right)$

3) se $t=1$, então $t^{\prime}=1, q=q^{\prime}$ e isto implica que $\Lambda([p, 1, q])=q=q^{\prime}=$ $\Lambda\left(\left[p^{\prime}, 1, q^{\prime}\right]\right)$.

Portanto, $\Lambda$ está bem definida.

Como a aplicação $\xi$ é contínua e $\pi$ é a projeção, segue que $\Lambda$ é contínua.

A aplicação $\Lambda$ é injetora. De fato, sejam $[p, t, q] \neq\left[p^{\prime}, t^{\prime}, q^{\prime}\right]$ duas classes distintas de $(P \times[0,1] \times Q) / \sim$. Suponhamos $\Lambda([p, t, q])=\Lambda\left(\left[p^{\prime}, t^{\prime}, q^{\prime}\right]\right)$, então $(1-t) p+t q=\left(1-t^{\prime}\right) p^{\prime}+t^{\prime} q^{\prime} \in P Q$. Como $P, Q$ são independentes, temos o seguinte.

1) Se $t \in(0,1)$, então $p=p^{\prime}, q=q^{\prime}, t=t^{\prime}$ e $[p, t, q]=\left[p^{\prime}, t^{\prime}, q^{\prime}\right]$. Contradição.

2) Se $t=0$, então $t^{\prime}=t=0, p=\Lambda([p, 0, q])=\Lambda\left(\left[p^{\prime}, 0, q^{\prime}\right]\right)=p^{\prime} \mathrm{e}$ $[p, 0, q]=\left[p^{\prime}, 0, q^{\prime}\right]$. Contradição.

3) Se $t=1$, então $t^{\prime}=t=1, q=\Lambda([p, 1, q])=\Lambda\left(\left[p^{\prime}, 1, q^{\prime}\right]\right)=q^{\prime}$ e $[p, 1, q]=\left[p^{\prime}, 1, q^{\prime}\right]$. Contradição. 
Portanto, $\Lambda$ é injetora.

A aplicação $\Lambda$ é sobrejetora. De fato, seja $y \in P Q$, então, pela Definição 1.1.1, existem $p \in P, t \in[0,1], q \in Q$ tais que $y=(1-t) p+t q=y$. Temos, portanto, que $\Lambda(\pi(p, t, q))=\Lambda([p, t, q])=(1-t) p+t q=y$.

Como $\Lambda$ é contínua e bijetora, $(P \times[0,1] \times Q) / \sim$ é compacto e $P Q$ é Hausdorff, segue que $\Lambda$ é um homeomorfismo [Li, pg.179].

Corolário 2.1.2 $P Q \backslash P$ é homeomorfo a $(P \times(0,1] \times Q) / \sim$.

\section{Demonstração:}

É suficiente mostrar que $\Lambda((P \times(0,1] \times Q) / \sim)=P Q \backslash P$. Para cada $x \in P Q \backslash P$, existem $\mu_{x} \in[0,1], p_{x} \in P, q_{x} \in Q$ tais que $x$ é da forma $\left(1-\mu_{x}\right) p_{x}+\mu_{x} q_{x}$, com $\mu_{x} \neq 0$. Portanto, $\Lambda^{-1}(x)=\left[p_{x}, \mu_{x}, q_{x}\right]$, com $\mu_{x} \neq 0$, ou seja, $\Lambda^{-1}(x) \in(P \times(0,1] \times Q) / \sim$. Por outro lado, se $[p, t, q] \in P * Q$, $\operatorname{com} t \neq 0$, então $\Lambda([p, t, q])=(1-t) p+t q$, com $t \neq 0$, e isto implica que $\Lambda([p, t, q]) \in P Q \backslash P$.

Proposição 2.1.3 Sejam $\sigma$ e $\tau$ simplexos. Então $\sigma$ e $\tau$ são independentes se, e somente se, seus vértices formam um conjunto independente.

\section{Demonstração:}

Sejam $\sigma=\left\langle v_{0} v_{1} \cdots v_{m}\right\rangle, \tau=\left\langle v_{m+1} v_{m+2} \cdots v_{n}\right\rangle$ dois simplexos. $(\Leftarrow)$ 
Suponhamos que exista um $x$ pertencente ao "join" $\sigma \tau$ que pode ser escrito de duas formas:

$$
\begin{array}{cl}
x=\lambda^{1} p^{1}+\mu^{1} q^{1}, \text { onde } & p^{1} \in \sigma, p^{1}=\sum_{i=0}^{m} \alpha_{i}^{1} v_{i}, \text { com } \alpha_{i}^{1} \geq 0, \sum_{i=0}^{m} \alpha_{i}^{1}=1 ; \\
& q^{1} \in \tau, q^{1}=\sum_{i=m+1}^{n} \alpha_{i}^{1} v_{i}, \operatorname{com} \alpha_{i}^{1} \geq 0, \sum_{i=m+1}^{n} \alpha_{i}^{1}=1 ; \\
\lambda^{1}, \mu^{1} \geq 0, \lambda^{1}+\mu^{1}=1,
\end{array}
$$

e

$$
\begin{gathered}
x=\lambda^{2} p^{2}+\mu^{2} q^{2}, \text { onde } \quad p^{2} \in \sigma, p^{2}=\sum_{i=0}^{m} \alpha_{i}^{2} v_{i}, \operatorname{com} \alpha_{i}^{2} \geq 0, \sum_{i=0}^{m} \alpha_{i}^{2}=1 ; \\
q^{2} \in \tau, q^{2}=\sum_{i=m+1}^{n} \alpha_{i}^{2} v_{i}, \operatorname{com} \alpha_{i}^{2} \geq 0, \sum_{i=m+1}^{n} \alpha_{i}^{2}=1 ; \\
\lambda^{2}, \mu^{2} \geq 0, \lambda^{2}+\mu^{2}=1 .
\end{gathered}
$$

Primeiro observemos que $\sum_{i=0}^{m} \alpha_{i}^{1}=1$, ou seja, $\sum_{i=0}^{m} \alpha_{i}^{1} v_{0}=v_{0}$, portanto podemos reescrever $p^{1}$ como sendo:

$$
p^{1}=v_{0}+\sum_{i=0}^{m} \alpha_{i}^{1} v_{i}-v_{0}=v_{0}+\sum_{i=0}^{m} \alpha_{i}^{1} v_{i}-\sum_{i=0}^{m} \alpha_{i}^{1} v_{0}=v_{0}+\sum_{i=1}^{m} \alpha_{i}^{1}\left(v_{i}-v_{0}\right) .
$$

Analogamente, podemos reescrever $q^{1}$ como sendo:

$$
q^{1}=v_{0}+\sum_{i=m+1}^{n} \alpha_{i}^{1} v_{i}-v_{0}=v_{0}+\sum_{i=m+1}^{n} \alpha_{i}^{1} v_{i}-\sum_{i=m+1}^{n} \alpha_{i}^{1} v_{0}=v_{0}+\sum_{i=m+1}^{n} \alpha_{i}^{1}\left(v_{i}-v_{0}\right) .
$$

Assim temos:

$$
\begin{aligned}
x & =\lambda^{1} p^{1}+\mu^{1} q^{1} \\
& =\lambda^{1}\left(v_{0}+\sum_{i=1}^{m} \alpha_{i}^{1}\left(v_{i}-v_{0}\right)\right)+\mu^{1}\left(v_{0}+\sum_{i=m+1}^{n} \alpha_{i}^{1}\left(v_{i}-v_{0}\right)\right) \\
& =\left(\lambda^{1}+\mu^{1}\right) v_{0}+\lambda^{1} \sum_{i=1}^{m} \alpha_{i}^{1}\left(v_{i}-v_{0}\right)+\mu^{1} \sum_{i=m+1}^{n} \alpha_{i}^{1}\left(v_{i}-v_{0}\right) \\
& =\left(\lambda^{1}+\mu^{1}\right) v_{0}+\sum_{i=1}^{n} \delta_{i}^{1}\left(v_{i}-v_{0}\right) \\
& =v_{0}+\sum_{i=1}^{n} \delta_{i}^{1}\left(v_{i}-v_{0}\right),
\end{aligned}
$$


e portanto,

$$
x=v_{0}+\sum_{i=1}^{n} \delta_{i}^{1}\left(v_{i}-v_{0}\right)
$$

onde $\delta_{i}^{1}=\lambda^{1} \alpha_{i}^{1}$, para $i=1, \ldots, m$ e $\delta_{i}^{1}=\mu^{1} \alpha_{i}^{1}$, para $i=m+1, \ldots, n$.

Repetindo as contas feitas em (2.2), podemos reescrever $x$ da seguinte forma:

$$
x=v_{0}+\sum_{i=1}^{n} \delta_{i}^{2}\left(v_{i}-v_{0}\right)
$$

onde $\delta_{i}^{2}=\lambda^{2} \alpha_{i}^{2}$, para $i=1, \ldots, m$ e $\delta_{i}^{2}=\mu^{2} \alpha_{i}^{2}$, para $i=m+1, \ldots, n$.

Como a seqüência de vetores

$$
\left\{v_{1}-v_{0}, \ldots, v_{m}-v_{0}, v_{m+1}-v_{0}, \ldots, v_{n}-v_{0}\right\}
$$

é linearmente independente e $(2.3)=(2.4)$, segue que:

$$
\delta_{i}^{1}=\delta_{i}^{2}, \forall i \in\{1, \ldots, n\} \Rightarrow\left\{\begin{array}{l}
\lambda^{1} \alpha_{i}^{1}=\lambda^{2} \alpha_{i}^{2}, \forall i \in\{1, \ldots, m\}, \\
\mu^{1} \alpha_{i}^{1}=\mu^{2} \alpha_{i}^{2}, \forall i \in\{m+1, \ldots, n\} .
\end{array}\right.
$$

Vamos primeiro mostrar que $\mu^{1}=\mu^{2}$. Por (2.5) sabemos que $\mu^{1} \alpha_{i}^{1}=$ $\mu^{2} \alpha_{i}^{2}, \forall i \in\{m+1, \ldots, n\}$. Portanto,

$$
\mu^{1}=\mu^{1} \sum_{i=m+1}^{n} \alpha_{i}^{1}=\sum_{i=m+1}^{n} \mu^{1} \alpha_{i}^{1}=\sum_{i=m+1}^{n} \mu^{2} \alpha_{i}^{2}=\mu^{2} \sum_{i=m+1}^{n} \alpha_{i}^{2}=\mu^{2}
$$

pois $\sum_{i=m+1}^{n} \alpha_{i}^{1}=\sum_{i=m+1}^{n} \alpha_{i}^{2}=1$

Como $\lambda^{1}+\mu^{1}=1=\lambda^{2}+\mu^{2}$, segue que $\lambda^{1}=\lambda^{2}$. Se $\lambda^{1}=\lambda^{2}>0$ e $\mu^{1}=\mu^{2}>0$, então $\alpha_{i}^{1}=\alpha_{i}^{2}$ para $i=1,2, \ldots, n$. Como $\sum_{i=0}^{m} \alpha_{i}^{1}=\sum_{i=0}^{m} \alpha_{i}^{2}=1$, temos também $\alpha_{0}^{1}=\alpha_{0}^{2}$. Por isso $p^{1}=p^{2}$ e $q^{1}=q^{2}$, e $\sigma$ e $\tau$ são independentes. 


\section{$(\Rightarrow)$}

Usando um argumento similar ao anterior, podemos provar esta implicação. No entanto, uma vez que este lema é bem conhecido, vamos omitir a sua demonstração.

\subsection{Alguns resultados sobre as fibras de uma aplicação simplicial entre simplexos}

Nesta seção efetuamos um estudo das fibras de aplicações simpliciais entre dois simplexos. A partir disso, na Seção 2.3, analisamos as fibras de aplicações entre complexos simpliciais.

Sejam $\sigma=\left\langle v_{0} v_{1} \cdots v_{m}\right\rangle$ um $m$-simplexo, $\tau=\left\langle w_{0} w_{1} \cdots w_{n}\right\rangle$ um $n$-simplexo, com $m \geq n$ e $f: \sigma \rightarrow \tau$ uma aplicação simplicial sobrejetora.

A aplicação $f$ fica definida sabendo-se apenas os valores das imagens dos vértices de $\sigma$.

Reordenando os índices dos vértices de $\sigma$, se necessário, de forma que:

$$
0 \leq i_{0}<i_{1}<\cdots<i_{n}=m
$$

$\mathrm{e}$

$$
\begin{gathered}
f\left(v_{0}\right)=f\left(v_{1}\right)=\cdots=f\left(v_{i_{0}}\right)=w_{0} \\
f\left(v_{i_{0}+1}\right)=f\left(v_{i_{0}+2}\right)=\cdots=f\left(v_{i_{1}}\right)=w_{1}, \\
\vdots \\
f\left(v_{i_{n-1}+1}\right)=f\left(v_{i_{n-1}+2}\right)=\cdots=f\left(v_{i_{n}}\right)=w_{n},
\end{gathered}
$$

temos que

$$
f^{-1}\left(w_{k}\right)=\left\{\sum_{i=i_{k-1}+1}^{i_{k}} \alpha_{i} v_{i}: \alpha_{i} \geq 0, \sum_{i=i_{k-1}+1}^{i_{k}} \alpha_{i}=1\right\},
$$

para $k=0, \ldots, n$, onde $i_{-1}=-1$. 


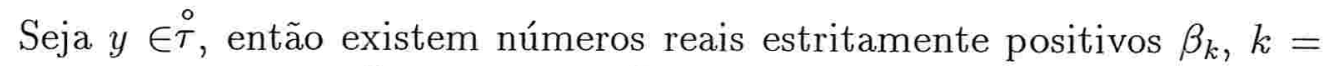
$0, \ldots, n$, tais que $y=\sum_{k=0}^{n} \beta_{k} w_{k}, \operatorname{com} \sum_{k=0}^{n} \beta_{k}=1$.

Consideremos um ponto qualquer $x$ pertencente à fibra $f^{-1}(y)$. Como $f^{-1}(y) \subset \sigma, x$ é da forma $\sum_{i=0}^{m} \alpha_{i} v_{i}, \operatorname{com} \alpha_{i} \geq 0$ e $\sum_{i=0}^{m} \alpha_{i}=1$. Pela definição da aplicação $f$, segue que:

$$
\begin{aligned}
f(x) & =f\left(\sum_{i=0}^{m} \alpha_{i} v_{i}\right)=f\left(\sum_{k=0}^{n} \sum_{i=i_{k-1}+1}^{i_{k}} \alpha_{i} v_{i}\right) \\
& =\sum_{k=0}^{n} \sum_{i=i_{k-1}+1}^{i_{k}} \alpha_{i} f\left(v_{i}\right)=\sum_{k=0}^{n} \sum_{i=i_{k-1}+1}^{i_{k}} \alpha_{i} w_{k} .
\end{aligned}
$$

Por outro lado,

$$
f(x)=y=\sum_{k=0}^{n} \beta_{k} w_{k} .
$$

Como $\left\{w_{0}, w_{1}, \ldots, w_{n}\right\}$ é um conjunto independente, ou seja,

$$
\left\{w_{1}-w_{0}, \ldots, w_{n}-w_{0}\right\}
$$

é linearmente independente, temos que $\beta_{k}=\sum_{i=i_{k-1}+1}^{i_{k}} \alpha_{i}$, para $k=0,1, \ldots, n$. Assim, as coordenadas baricêntricas de $y$ e de um ponto qualquer $x \in f^{-1}(y)$ satisfazem a seguinte relação:

$$
\left\{\begin{aligned}
\beta_{0} & =\alpha_{0}+\alpha_{1}+\cdots+\alpha_{i_{0}} \\
\beta_{1} & =\alpha_{i_{0}+1}+\alpha_{i_{0}+2}+\cdots+\alpha_{i_{1}} \\
& \vdots \\
\beta_{n} & =\alpha_{i_{n-1}+1}+\alpha_{i_{n-1}+2}+\cdots+\alpha_{i_{n}} .
\end{aligned}\right.
$$

Utilizando as notações acima, temos a seguinte:

Proposição 2.2.1 Sejam $\sigma=\left\langle v_{0} v_{1} \cdots v_{m}\right\rangle$ e $\tau=\left\langle w_{0} w_{1} \cdots w_{n}\right\rangle$ dois simplexos, $m \geq n$, e $f: \sigma \rightarrow \tau$ uma aplicação simplicial sobrejetora. Então, 
para cada $y \in \stackrel{\circ}{\tau}$, a fibra $f^{-1}(y)$ é homeomorfa a $\sigma_{0} \times \cdots \times \sigma_{n}$, onde $\sigma_{k}$ é um $\left(i_{k}-i_{k-1}-1\right)$-simplexo.

\section{Demonstração:}

Para cada índice $k=0,1, \ldots, n$, consideremos $\sigma_{k}$ o simplexo de vértices $v_{i_{k-1}+1}, v_{i_{k-1}+2}, \ldots, v_{i_{k}}$.

A aplicação $h_{k}: f^{-1}(y) \longrightarrow \sigma_{k}$ definida por

$$
h_{k}(x)=\frac{1}{\beta_{k}} \sum_{i=i_{k-1}+1}^{i_{k}} \alpha_{i} v_{i}
$$

onde $x=\sum_{i=0}^{m} \alpha_{i} v_{i}=\sum_{k=0}^{n} \sum_{i=i_{k-1}+1}^{i_{k}} \alpha_{i} v_{i} \in f^{-1}(y), \operatorname{com} \alpha_{i} \geq 0$ e $\sum_{i=0}^{m} \alpha_{i}=1$, está bem definida, pois $\frac{\alpha_{i}}{\beta_{k}} \geq 0$, para $\forall i$ e $\forall k$. Mais ainda,

$$
\sum_{i=i_{k-1}+1}^{i_{k}} \frac{\alpha_{i}}{\beta_{k}}=\left(\frac{1}{\sum_{i=i_{k-1}+1}^{i_{k}} \alpha_{i}}\right) \sum_{i=i_{k-1}+1}^{i_{k}} \alpha_{i}=1
$$

ou seja, $h_{k}(x) \in \sigma_{k}$. Notemos, ainda, que definida desta forma, $h_{k}$ é uma aplicação contínua.

Portanto, a aplicação $h: f^{-1}(y) \longrightarrow \sigma_{0} \times \cdots \times \sigma_{n}$ definida por $h(x)=$ $\left(h_{0}(x), \ldots, h_{n}(x)\right)$ está bem definida e é contínua.

Consideremos agora a aplicação $l: \sigma_{0} \times \cdots \times \sigma_{n} \longrightarrow f^{-1}(y)$ definida por

$$
l\left(\sum_{i=0}^{i_{0}} \delta_{i} v_{i}, \ldots, \sum_{i=i_{n-1}+1}^{i_{n}} \delta_{i} v_{i}\right)=\sum_{i=0}^{i_{0}} \beta_{0} \delta_{i} v_{i}+\cdots+\sum_{i=i_{n-1}+1}^{i_{n}} \beta_{n} \delta_{i} v_{i}
$$

onde $\delta_{i} \geq 0, \sum_{i=0}^{i_{0}} \delta_{i}=1, \ldots, \sum_{i=i_{n-1}+1}^{i_{n}} \delta_{i}=1$. A aplicação $l$ está bem definida, 
pois $\beta_{k} \delta_{i} \geq 0, \sum_{i=i_{k-1}+1}^{i_{k}} \beta_{k} \delta_{i}=\beta_{k}, \sum_{k=0}^{n} \beta_{k}=1$, ou seja,

$$
\begin{aligned}
\sum_{i=0}^{i_{0}} \beta_{0} \delta_{i}+\cdots+\sum_{i=i_{n-1}+1}^{i_{n}} \beta_{n} \delta_{i} & =\sum_{k=0}^{n} \sum_{i=i_{k-1}+1}^{i_{k}} \beta_{k} \delta_{i} \\
& =\sum_{k=0}^{n} \beta_{k} \sum_{i=i_{k-1}+1}^{i_{k}} \delta_{i} \\
& =\sum_{k=0}^{n} \beta_{k} \\
& =1
\end{aligned}
$$

e isto implica que a imagem de $l$ está contida em $f^{-1}(y)$. Temos ainda que $l$ é contínua.

A aplicação $l$ é a inversa de $h$. De fato, para todo

$$
\left(\sum_{i=0}^{i_{0}} \delta_{i} v_{i}, \ldots, \sum_{i=i_{n-1}+1}^{i_{n}} \delta_{i} v_{i}\right) \in \sigma_{0} \times \cdots \times \sigma_{n}
$$

temos:

$$
\begin{aligned}
h \circ l\left(\sum_{i=0}^{i_{0}} \delta_{i} v_{i}, \ldots, \sum_{i=i_{n-1}+1}^{i_{n}} \delta_{i} v_{i}\right)=h\left(\sum_{i=0}^{i_{0}} \beta_{0} \delta_{i} v_{i}+\cdots+\sum_{i=i_{n-1}+1}^{i_{n}} \beta_{n} \delta_{i} v_{i}\right) \\
=\left(h_{0}\left(\sum_{i=0}^{i_{0}} \beta_{0} \delta_{i} v_{i}+\cdots+\sum_{i=i_{n-1}+1}^{i_{n}} \beta_{n} \delta_{i} v_{i}\right), \ldots, h_{n}\left(\sum_{i=0}^{i_{0}} \beta_{0} \delta_{i} v_{i}+\cdots+\sum_{i=i_{n-1}+1}^{i_{n}} \beta_{n} \delta_{i} v_{i}\right)\right) \\
=\left(\frac{1}{\beta_{0}} \sum_{i=0}^{i_{0}} \beta_{0} \delta_{i} v_{i}, \ldots, \frac{1}{\beta_{n}} \sum_{i=i_{n-1}+1}^{i_{n}} \beta_{n} \delta_{i} v_{i}\right)=\left(\sum_{i=0}^{i_{0}} \delta_{i} v_{i}, \ldots, \sum_{i=i_{n-1}+1}^{i_{n}} \delta_{i} v_{i}\right) . \\
\quad \text { E para todo } x=\sum_{k=0}^{n} \sum_{i=i_{k-1}+1}^{i_{k}} \alpha_{i} v_{i} \in f^{-1}(y), \text { segue que: }
\end{aligned}
$$




$$
\begin{aligned}
l \circ h(x) & =l \circ h\left(\sum_{k=0}^{n} \sum_{i=i_{k-1}+1}^{i_{k}} \alpha_{i} v_{i}\right)=l\left(\frac{1}{\beta_{0}} \sum_{i=0}^{i_{0}} \alpha_{i} v_{i}, \ldots, \frac{1}{\beta_{n}} \sum_{i=i_{n-1}+1}^{i_{n}} \alpha_{i} v_{i}\right) \\
& =\frac{1}{\beta_{0}} \sum_{i=0}^{i_{0}} \beta_{0} \alpha_{i} v_{i}+\cdots+\frac{1}{\beta_{n}} \sum_{i=i_{n-1}+1}^{i_{n}} \beta_{n} \alpha_{i} v_{i} \\
& =\sum_{i=0}^{i_{0}} \alpha_{i} v_{i}+\cdots+\sum_{i=i_{n-1}+1}^{i_{n}} \alpha_{i} v_{i} \\
& =\sum_{k=0}^{n} \sum_{i=i_{k-1}+1}^{i_{k}} \alpha_{i} v_{i} \\
& =x .
\end{aligned}
$$

Portanto, $h$ é a inversa de $l$ e $h$ é o homeomorfismo procurado.

Corolário 2.2.2 Nas mesmas hipóteses anteriores, temos que para cada y ponto interior de $\tau$, a fibra $f^{-1}(y)$ é conexa.

\section{Demonstração:}

Para cada $j=0, \ldots, n$, temos que $\sigma_{j}$ é um simplexo, que é conexo, então $\sigma_{0} \times \cdots \times \sigma_{n}$ é conexo. Portanto, como $f^{-1}(y)$ é homeomorfa a $\sigma_{0} \times \cdots \times \sigma_{n}$, segue que $f^{-1}(y)$ é conexa.

Definição 2.2.3 A proposição anterior nos permite definir a dimensão da fibra $f^{-1}(y)$ da maneira mais natural possível, ou seja,

$$
\operatorname{dim}\left(f^{-1}(y)\right)=\operatorname{dim}\left(\sigma_{0} \times \cdots \times \sigma_{n}\right)=\sum_{k=0}^{n} \operatorname{dim} \sigma_{k} .
$$

O corolário abaixo nos dá a dimensão da fibra $f^{-1}(y)$.

Corolário 2.2.4 Sejam $\sigma=\left\langle v_{0} v_{1} \cdots v_{m}\right\rangle$ e $\tau=\left\langle w_{0} w_{1} \cdots w_{n}\right\rangle$ dois simplexos, $m \geq n$, e $f: \sigma \rightarrow \tau$ uma aplicação simplicial sobrejetora. Se y é um ponto interior do simplexo $\tau$, então a dimensão da fibra $f^{-1}(y)$ é igual a $m-n$. 


\section{Demonstração:}

Pela Proposição 2.2.1, temos que $f^{-1}(y)$ é homeomorfa a $\sigma_{0} \times \cdots \times \sigma_{n}$. Portanto,

$$
\begin{aligned}
\operatorname{dim}\left(f^{-1}(y)\right) & =\operatorname{dim}\left(\sigma_{0} \times \cdots \times \sigma_{n}\right) \\
& =\sum_{k=0}^{n} \operatorname{dim} \sigma_{k} \\
& =\sum_{k=0}^{n}\left(i_{k}-\left(i_{k-1}+1\right)\right) \\
& =\sum_{k=0}^{n}\left(i_{k}-i_{k-1}-1\right) \\
& =\sum_{k=0}^{n}\left(i_{k}-i_{k-1}\right)+\sum_{k=0}^{n}(-1) \\
& =i_{n}-i_{-1}+(-n-1) \\
& =m-(-1)-n-1 \\
& =m+1-n-1 \\
& =m-n .
\end{aligned}
$$

Exemplo 2.2.5 Consideremos os simplexos $\sigma=\left\langle v_{0} v_{1} v_{2}\right\rangle, \tau=\left\langle w_{0} w_{1}\right\rangle \mathrm{e}$ $f: \sigma \rightarrow \tau$ aplicação simplicial sobrejetora definida por:

$$
\left\{\begin{array}{l}
f\left(v_{0}\right)=w_{0} \\
f\left(v_{1}\right)=f\left(v_{2}\right)=w_{1} .
\end{array}\right.
$$

Sejam $\sigma_{0}=\left\langle v_{0}\right\rangle$ e $\sigma_{1}=\left\langle v_{1} v_{2}\right\rangle$. Então, se $y \in \stackrel{\circ}{\tau}$, temos que $f^{-1}(y)$ é homeomorfo a $\sigma_{0} \times \sigma_{1}$ e a dimensão de $f^{-1}(y)$ é igual a $2-1=1$.
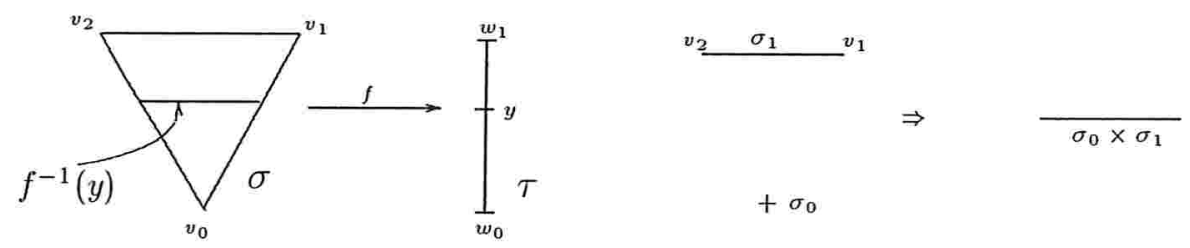
Exemplo 2.2.6 Consideremos os simplexos $\sigma=\left\langle v_{0} v_{1} v_{2} v_{3}\right\rangle, \tau=\left\langle w_{0} w_{1}\right\rangle$ e $f: \sigma \rightarrow \tau$ aplicação simplicial sobrejetora definida por:

$$
\left\{\begin{array}{l}
f\left(v_{0}\right)=f\left(v_{1}\right)=w_{0} \\
f\left(v_{2}\right)=f\left(v_{3}\right)=w_{1} .
\end{array}\right.
$$

Sejam $\sigma_{0}=\left\langle v_{0} v_{1}\right\rangle$ e $\sigma_{1}=\left\langle v_{2} v_{3}\right\rangle$. Então, se $y \in \stackrel{\circ}{\tau}$, temos que $f^{-1}(y)$ é homeomorfo a $\sigma_{0} \times \sigma_{1}$ e $\operatorname{dim}\left(f^{-1}(y)\right)=3-1=2$.
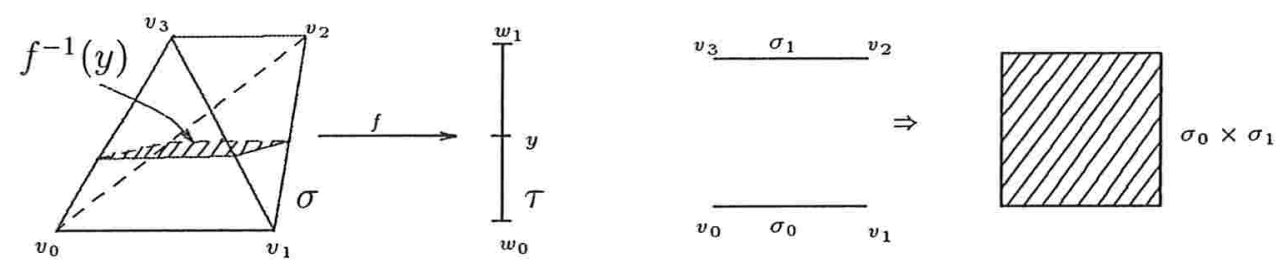

Exemplo 2.2.7 Consideremos os simplexos $\sigma=\left\langle v_{0} v_{1} v_{2} v_{3}\right\rangle, \tau=\left\langle w_{0} w_{1}\right\rangle$ e $f: \sigma \rightarrow \tau$ aplicação simplicial sobrejetora definida por:

$$
\left\{\begin{array}{l}
f\left(v_{0}\right)=f\left(v_{1}\right)=f\left(v_{2}\right)=w_{0}, \\
f\left(v_{3}\right)=w_{1}
\end{array}\right.
$$

Sejam $\sigma_{0}=\left\langle v_{0} v_{1} v_{2}\right\rangle$ e $\sigma_{1}=\left\langle v_{3}\right\rangle$. Então, se $y \in \stackrel{\circ}{\tau}$, temos que $f^{-1}(y)$ é homeomorfo a $\sigma_{0} \times \sigma_{1}$ e $\operatorname{dim}\left(f^{-1}(y)\right)=3-1=2$.
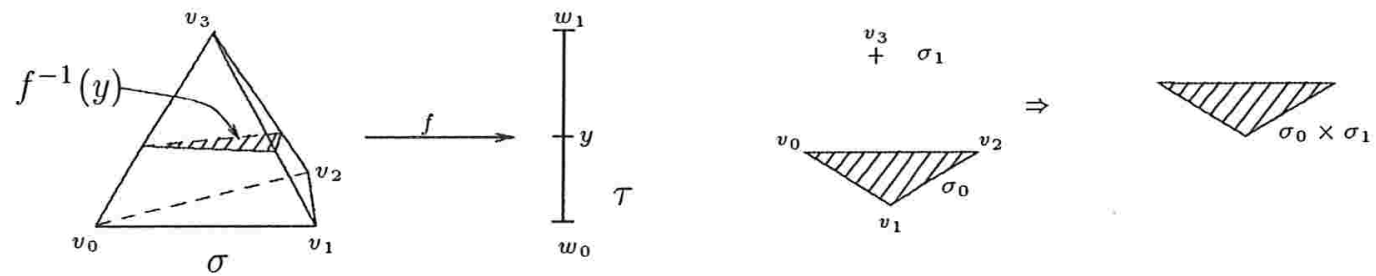
Exemplo 2.2.8 Consideremos os simplexos $\sigma=\left\langle v_{0} v_{1} \cdots v_{9}\right\rangle, \tau=\left\langle w_{0} w_{1} w_{2} w_{3}\right\rangle$ e $f: \sigma \rightarrow \tau$ uma aplicação simplicial sobrejetora definida por:

$$
\left\{\begin{array}{l}
f\left(v_{0}\right)=f\left(v_{1}\right)=w_{0}, \\
f\left(v_{2}\right)=f\left(v_{3}\right)=f\left(v_{4}\right)=w_{1}, \\
f\left(v_{5}\right)=w_{2}, \\
f\left(v_{6}\right)=f\left(v_{7}\right)=f\left(v_{8}\right)=f\left(v_{9}\right)=w_{3} .
\end{array}\right.
$$

Sejam $\sigma_{0}=\left\langle v_{0} v_{1}\right\rangle, \sigma_{1}=\left\langle v_{2} v_{3} v_{4}\right\rangle, \sigma_{2}=\left\langle v_{5}\right\rangle$ e $\sigma_{3}=\left\langle v_{6} v_{7} v_{8} v_{9}\right\rangle$. Então, se $y \in \stackrel{\circ}{\tau}$, temos que $f^{-1}(y)$ é homeomorfo a $\sigma_{0} \times \sigma_{1} \times \sigma_{2} \times \sigma_{3}$ e $\operatorname{dim}\left(f^{-1}(y)\right)=$ $9-3=6$.
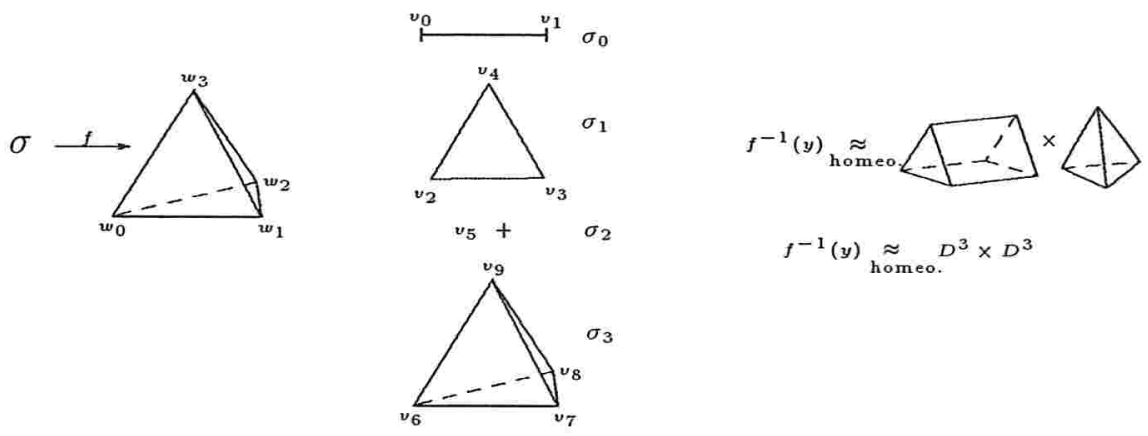

Corolário 2.2.9 Sejam $\sigma=\left\langle v_{0} v_{1} \cdots v_{m}\right\rangle$ e $\tau=\left\langle w_{0} w_{1} \cdots w_{n}\right\rangle$ dois simple$x o s, m \geq n$, e $f: \sigma \rightarrow \tau$ uma aplicação simplicial sobrejetora. Se $y^{1} e$ $y^{2}$ são dois pontos do interior de $\tau$, então as fibras $f^{-1}\left(y^{1}\right)$ e $f^{-1}\left(y^{2}\right)$ são homeomorfas.

\section{Demonstração:}

Seguindo os mesmo passos da demonstração da Proposição 2.2.1, consideremos $y^{1}=\sum_{k=0}^{n} \beta_{k}^{1} w_{k}$ e $y^{2}=\sum_{k=0}^{n} \beta_{k}^{2} w_{k}$, com $\sum_{k=0}^{n} \beta_{k}^{i}=1$ e $\beta_{k}^{i}>0$, para qualquer $k \in\{0, \ldots, n\}$ e $i=1,2$.

Consideremos as aplicações $h_{1}: f^{-1}\left(y^{1}\right) \longrightarrow \sigma_{0} \times \cdots \times \sigma_{n}$ definida por

$$
h_{1}\left(x^{1}\right)=\left(\frac{1}{\beta_{0}^{1}} \sum_{i=0}^{i_{0}} \alpha_{i}^{1} v_{i}, \ldots, \frac{1}{\beta_{n}^{1}} \sum_{i=i_{n-1}+1}^{i_{n}} \alpha_{i}^{1} v_{i}\right) \text {, }
$$


onde $x^{1}=\sum_{i=0}^{m} \alpha_{i}^{1} v_{i}, \operatorname{com} \alpha_{i}^{1} \geq 0, \sum_{i=i_{k-1}+1}^{i_{k}} \alpha_{i}^{1}=\beta_{k}^{1}, k=0, \ldots, n$ e $i_{-1}=-1$, e $h_{2}: f^{-1}\left(y^{2}\right) \longrightarrow \sigma_{0} \times \cdots \times \sigma_{n}$ definida por

$$
h_{2}\left(x^{2}\right)=\left(\frac{1}{\beta_{0}^{2}} \sum_{i=0}^{i_{0}} \alpha_{i}^{2} v_{i}, \ldots, \frac{1}{\beta_{n}^{2}} \sum_{i=i_{n-1}+1}^{i_{n}} \alpha_{i}^{2} v_{i}\right) \text {, }
$$

onde $x^{2}=\sum_{i=0}^{m} \alpha_{i}^{2} v_{i}, \operatorname{com} \alpha_{i}^{2} \geq 0, \sum_{i=i_{k-1}+1}^{i_{k}} \alpha_{i}^{2}=\beta_{k}^{2}, k=0, \ldots, n$ e $i_{-1}=-1$.

Notemos que $h_{1}$ e $h_{2}$ são homeomorfismos.

Consideremos a inversa $h_{2}^{-1}: \sigma_{0} \times \cdots \times \sigma_{n} \longrightarrow f^{-1}\left(y^{2}\right)$ de $h_{2}$ definida por

$$
h_{2}^{-1}\left(\sum_{i=0}^{i_{0}} \delta_{i} v_{i}, \ldots, \sum_{i=i_{n-1}+1}^{i_{n}} \delta_{i} v_{i}\right)=\sum_{i=0}^{i_{0}} \beta_{0}^{2} \delta_{i} v_{i}+\cdots+\sum_{i=i_{n-1}+1}^{i_{n}} \beta_{n}^{2} \delta_{i} v_{i} .
$$

Então $h:=h_{2}^{-1} \circ h_{1}: f^{-1}\left(y^{1}\right) \longrightarrow f^{-1}\left(y^{2}\right)$ definida por

$$
\begin{aligned}
h\left(x^{1}\right) & =h_{2}^{-1}\left(h_{1}\left(x^{1}\right)\right) \\
& =h_{2}^{-1}\left(\frac{1}{\beta_{0}^{1}} \sum_{i=0}^{i_{0}} \alpha_{i}^{1} v_{i}, \ldots, \frac{1}{\beta_{n}^{1}} \sum_{i=i_{n-1}+1}^{i_{n}} \alpha_{i}^{1} v_{i}\right) \\
& =\sum_{i=0}^{i_{0}}\left(\frac{\beta_{0}^{2}}{\beta_{0}^{1}} \alpha_{i}^{1} v_{i}\right)+\cdots+\sum_{i=i_{n-1}+1}^{i_{n}}\left(\frac{\beta_{n}^{2}}{\beta_{n}^{1}} \alpha_{i}^{1} v_{i}\right)
\end{aligned}
$$

é um homeomorfismo entre as fibras $f^{-1}\left(y^{1}\right)$ e $f^{-1}\left(y^{2}\right)$.

\subsection{Um estudo sobre as fibras de uma apli- cação simplicial entre complexos}

O objetivo desta seção é o estudo das fibras de uma aplicação simplicial $f: K \rightarrow L$ entre complexos simpliciais. Vamos primeiro examinar alguns lemas. 
Lema 2.3.1 Sejam $\sigma, \tau$ dois simplexos e $f: \sigma \rightarrow \tau$ uma aplicação simplicial. Então, são equivalentes:

(1) $f^{(0)}: \sigma^{(0)} \longrightarrow \tau^{(0)}$ é sobrejetora;

(2) $f: \sigma \rightarrow \tau$ é sobrejetora;

(3) $f(\stackrel{\circ}{\sigma}) \subset \stackrel{\circ}{\tau}$;

(4) $\theta \ll \tau \Rightarrow f^{-1}(\theta) \ll \sigma$.

\section{Demonstração:}

Sejam $\sigma=\left\langle v_{0} v_{1} \cdots v_{m}\right\rangle, \tau=\left\langle w_{0} w_{1} \cdots w_{n}\right\rangle$ e $f: \sigma \rightarrow \tau$ definida por $f\left(v_{i_{k-1}+1}\right)=\cdots=f\left(v_{i_{k}}\right)=w_{k}, k \in\{0, \ldots, n\}$, onde $-1 \leq i_{0} \leq \cdots \leq i_{n}=m$ e $i_{-1}=-1$.

$(1) \Rightarrow(2)$ Seja $y \in \tau, y=\sum_{k=0}^{n} \beta_{k} w_{k}$. Como $f^{(0)}$ é sobrejetora, temos que $\left(f^{(0)}\right)^{-1}\left(w_{k}\right) \neq \emptyset, \forall k \in\{0,1, \ldots, n\}$, i.e., $0 \leq i_{0}<i_{1}<\cdots<i_{n}$. Consideremos $x \in \sigma$ tal que $x=\sum_{k=0}^{n} \sum_{i=i_{k-1}+1}^{i_{k}} \alpha_{i} v_{i}$, onde $\alpha_{i} \geq 0$ são números reais tais que $\sum_{i=i_{k-1}+1}^{i_{k}} \alpha_{i}=\beta_{k}$, para $k=0,1, \ldots, n$. Note que um tal ponto $x \in \sigma$ sempre existe. Temos que $f(x)=\sum_{k=0}^{n} \sum_{i=i_{k-1}+1}^{i_{k}} \alpha_{i} f\left(v_{i}\right)=$ $\sum_{k=0}^{n} \beta_{k} w_{k}=y$. Portanto, $f$ é sobrejetora.

$(2) \Rightarrow(1)$ Suponha $f^{(0)}$ não sobrejetora, então existe $w_{k_{0}} \in \tau^{(0)}$ tal que $f\left(v_{i}\right) \neq w_{k_{0}}, \forall i \in\{0,1, \ldots, m\}$. Seja $y \in \stackrel{\circ}{\tau}$, temos que $y=\sum_{k=0}^{n} \beta_{k} w_{k}$, $\operatorname{com} \sum_{k=0}^{n} \beta_{k}=1$ e $\beta_{k}>0, \forall k$. Mas, para todo $x \in \sigma$, temos que $x=\sum_{i=0}^{m} \alpha_{i} v_{i}$ e $f(x)=\sum_{i=0}^{m} \alpha_{i} f\left(v_{i}\right)$, onde $f\left(v_{i}\right) \neq w_{k_{0}}, \forall i$. Segue que 
não existe $x \in \sigma$ tal que $f(x)=y$, o que é uma contradição, pois $f$ é sobrejetora. Portanto, $f^{(0)}$ também é sobrejetora.

$(3) \Rightarrow(2)$ Suponhamos $f$ não sobrejetora. Pelo item (1), temos que $f^{(0)}$ também não é sobrejetora, então existe $w_{k_{0}}$, vértice de $\tau$, tal que $f\left(v_{i}\right) \neq w_{k_{0}}, \forall i \in\{0, \ldots, m\}$. Consideremos $x \in \stackrel{\circ}{\sigma}$, então $x=\sum_{i=0}^{m} \alpha_{i} v_{i}$ e $f(x)=\sum_{i=0}^{m} \alpha_{i} f\left(v_{i}\right)=\sum_{\substack{k=0 \\ k \neq k_{0}}}^{m} \sum_{i=i_{k-1}+1}^{i_{k}} \alpha_{i} w_{k}$. Segue que $f(x) \notin \stackrel{\circ}{\tau}$, pois o coeficiente de $w_{k_{0}}$ é igual a zero, o que é uma contradição. Temos então que o item (2) está satisfeito.

(4) $\Rightarrow(3)$ Suponha que exista $x \in \stackrel{\circ}{\sigma}$ tal que $f(x) \notin \stackrel{\circ}{\tau}$. Então existe uma face própria $\theta$ de $\tau$ tal que $f(x) \in \theta$. Pelo item (4), temos que $x \in$ $f^{-1}(f(x)) \subset f^{-1}(\theta) \ll \sigma$. Contradição, pois $x$ é um ponto do interior de $\sigma$. Portanto, $f(x) \in \stackrel{\circ}{\tau}, \forall x \in \stackrel{\circ}{\sigma}$, ou seja, $f(\stackrel{\circ}{\sigma}) \subset \stackrel{\circ}{\tau}$.

(2) $\Rightarrow(4)$ Seja $\theta=\left\langle w_{k_{1}} \cdots w_{k_{r}}\right\rangle$ uma face própria do simplexo $\tau$. É fácil mostrar que $f^{-1}(\theta) \supset\left\langle v_{i_{k_{1}-1}+1} \cdots v_{i_{k_{1}}} \cdots v_{i_{k_{r}-1}+1} \cdots v_{i_{k_{r}}}\right\rangle$. A outra inclusão se mostra através da noção de coordenadas baricêntricas. Portanto, $f^{-1}(\theta)=\left\langle v_{i_{k_{1}-1}+1} \cdots v_{i_{k_{1}}} \cdots v_{i_{k_{r}-1}+1} \cdots v_{i_{k_{r}}}\right\rangle$. Como $\theta$ é uma face própria de $\tau$, existe $w_{l}$ vértice de $\tau$ tal que $l \notin\left\{k_{1}, \ldots, k_{r}\right\}$, ou seja, $w_{l} \notin \theta$. Como $f$ é sobrejetora, temos $f^{-1}\left(w_{l}\right) \neq \emptyset$ e $v_{s} \notin f^{-1}(\theta)$, para todo $s \in\left\{i_{l-1}+1, \ldots, i_{l}\right\}$. Portanto, $f^{-1}(\theta)$ também é uma face própria de $\sigma$.

Lema 2.3.2 Sejam $\sigma$ um m-simplexo, $\tau$ um n-simplexo e $f: \sigma \rightarrow \tau$ uma aplicação simplicial. Se, para alguma face $\theta$ de $\sigma$, tivermos $f^{-1}(y) \cap \theta \neq \emptyset$ com $y \in \stackrel{\circ}{\tau}$, então $\left.f\right|_{\theta}$ é sobrejetora e $m \geq n$. 


\section{Demonstração:}

Suponhamos que $\left.f\right|_{\theta}$ não seja sobrejetora, então pelo Lema 2.3 .1 existe um vértice $w_{k_{0}}$ de $\tau$ tal que $f(v) \neq w_{k_{0}}$, para todo vértice $v$ de $\theta$.

Seja $x \in f^{-1}(y) \cap \theta$. Existem números reais não negativos $\alpha_{i}, i=0, \ldots, r$, com $r \leq m$, tais que $x=\sum_{i=0}^{r} \alpha_{i} v_{i}$, onde $v_{0}, \ldots, v_{r}$ são os vértices de $\theta$.

Mas $y=f(x)=f\left(\sum_{i=0}^{r} \alpha_{i} v_{i}\right)=\sum_{i=0}^{r} \alpha_{i} f\left(v_{i}\right)$. Como $f\left(v_{i}\right) \neq w_{k_{0}}$, para qualquer $i \in\{0, \ldots, r\}$, temos que a coordenada baricêntrica correspondente a $w_{k_{0}}$ é zero, o que é uma contradição, pois $y$ é um ponto interior de $\tau$.

Portanto, $\left.f\right|_{\theta}$ é sobrejetora e, conseqüentemente, $m \geq r \geq n$.

Dada uma aplicação simplicial $f: K \rightarrow L$ entre complexos simpliciais, nosso objetivo é comparar duas fibras correspondentes a dois pontos distintos do interior de um mesmo simplexo de $L$, portanto consideramos, em algumas proposições, aplicações simpliciais definidas em $K$ com imagem em um simplexo $\tau$.

Dada uma aplicação simplicial $f: K \rightarrow \tau$, onde $K$ é um complexo simplicial e $\tau$ é um simplexo, o Lema 2.3 .2 diz que se $y \in \stackrel{\circ}{\tau}$ e $f^{-1}(y) \cap$ $\sigma \neq \emptyset$, para algum $\sigma \in K$, então $f$ é sobrejetora. Por outro lado, nas mesmas condições acima, o Corolário 2.2 .9 diz que se $y^{1}, y^{2} \in \stackrel{\circ}{\tau}$, então as fibras $f^{-1}\left(y^{1}\right) \cap \sigma$ e $f^{-1}\left(y^{2}\right) \cap \sigma$ são homeomorfas. Seja $h_{\sigma}: f^{-1}\left(y^{1}\right) \cap \sigma \rightarrow$ $f^{-1}\left(y^{2}\right) \cap \sigma$ o homeomorfismo dado na demonstração do Corolário 2.2.9. Considere agora o seguinte:

Lema 2.3.3 Sejam $K$ um complexo simplicial, $\tau$ um $n$-simplexo, $f: K \rightarrow \tau$ uma aplicação simplicial sobrejetora e $y^{1}, y^{2} \in \stackrel{\circ}{\tau}$. Se $\theta<\sigma$ são dois simplexos de $K$ tais que $f^{-1}\left(y^{1}\right) \cap \sigma \neq \emptyset$ e $f^{-1}\left(y^{1}\right) \cap \theta \neq \emptyset$, então $\left.h_{\sigma}\right|_{f^{-1}\left(y^{1}\right) \cap \theta}=h_{\theta}: f^{-1}\left(y^{1}\right) \cap \theta \rightarrow f^{-1}\left(y^{2}\right) \cap \theta$. 


\section{Demonstração:}

Sejam $\sigma=\left\langle v_{0} v_{1} \cdots v_{m}\right\rangle, \tau=\left\langle w_{0} w_{1} \cdots w_{n}\right\rangle$. Utilizaremos as mesmas notações introduzidas na demonstração do Corolário 2.2.9.

Consideremos $y^{1}=\sum_{k=0}^{n} \beta_{k}^{1} w_{k}, y^{2}=\sum_{k=0}^{n} \beta_{k}^{2} w_{k}$, onde $\sum_{k=0}^{n} \beta_{k}^{j}=1, \beta_{k}^{j}>0$, $\forall k \in\{0,1, \ldots, n\}, j=1,2 ;$ e seja $x \in f^{-1}\left(y^{1}\right) \cap \sigma$, isto é, $x=\sum_{i=0}^{m} \alpha_{i} v_{i}$, com $\alpha_{i} \geq 0, \sum_{i=i_{k-1}+1}^{i_{k}} \alpha_{i}=\beta_{k}^{1}, k \in\{0, \ldots, n\}, i_{-1}=-1$.

Seja o homeomorfismo $h_{\sigma}: f^{-1}\left(y^{1}\right) \cap \sigma \longrightarrow f^{-1}\left(y^{2}\right) \cap \sigma$ dado por

$$
h_{\sigma}(x)=\sum_{i=0}^{i_{0}}\left(\frac{\beta_{0}^{2}}{\beta_{0}^{1}} \alpha_{i} v_{i}\right)+\cdots+\sum_{i=i_{n-1}+1}^{i_{n}}\left(\frac{\beta_{n}^{2}}{\beta_{n}^{1}} \alpha_{i} v_{i}\right) \text {. }
$$

Seja $\theta=\left\langle v_{l_{0}} v_{l_{1}} \cdots v_{l_{r}}\right\rangle$ uma $r$-face de $\sigma$, com $f^{-1}\left(y^{1}\right) \cap \theta \neq \emptyset$. Pelo Lema 2.3.2, $\left.f\right|_{\theta}: \theta \longrightarrow \tau$ é sobrejetora e, pelo Corolário 2.2.9, temos que $f^{-1}\left(y^{2}\right) \cap \theta \neq \emptyset$ e existe um homeomorfismo $h_{\theta}: f^{-1}\left(y^{1}\right) \cap \theta \longrightarrow f^{-1}\left(y^{2}\right) \cap \theta$ definido da seguinte forma: se $z \in f^{-1}\left(y^{1}\right) \cap \theta$, então $z=\sum_{j \in J} \delta_{j} v_{j}$, onde $\delta_{j} \geq 0$, para $j \in J, \sum_{j \in J} \delta_{j}=1$ e $J=\left\{l_{0}, l_{1}, \ldots, l_{r}\right\}$. Seja ainda $J_{k}=$ $J \cap\left\{i_{k-1}+1, \ldots, i_{k}\right\} \neq \emptyset, k=0,1, \ldots, n$, então, necessariamente temos que $\sum_{j \in J_{k}} \delta_{j}=\beta_{k}^{1}, k \in\{0, \ldots, n\}$. Portanto,

$$
h_{\theta}(z)=\sum_{j \in J_{0}}\left(\frac{\beta_{0}^{2}}{\beta_{0}^{1}} \delta_{j} v_{j}\right)+\cdots+\sum_{j \in J_{n}}\left(\frac{\beta_{n}^{2}}{\beta_{n}^{1}} \delta_{j} v_{j}\right)
$$

e $\left.h_{\sigma}\right|_{f^{-1}\left(y^{1}\right) \cap \theta}=h_{\theta}$. 
Exemplo 2.3.4 Sejam $\sigma=\left\langle v_{0} v_{1} v_{2} v_{3} v_{4} v_{5} v_{6}\right\rangle, \theta=\left\langle v_{1} v_{3} v_{4} v_{6}\right\rangle$ uma 3-face de $\sigma, \tau=\left\langle w_{0} w_{1} w_{2}\right\rangle$ e $f: \sigma \rightarrow \tau$ a aplicação simplicial sobrejetora definida por:

$$
\left\{\begin{array}{l}
f\left(v_{0}\right)=f\left(v_{1}\right)=f\left(v_{2}\right)=w_{0} \\
f\left(v_{3}\right)=f\left(v_{4}\right)=w_{1} \\
f\left(v_{5}\right)=f\left(v_{6}\right)=w_{2}
\end{array}\right.
$$

Consideremos os pontos $y^{1}=\beta_{0}^{1} w_{0}+\beta_{1}^{1} w_{1}+\beta_{2}^{1} w_{2}$ e $y^{2}=\beta_{0}^{2} w_{0}+\beta_{1}^{2} w_{1}+$ $\beta_{2}^{2} w_{2}$ em $\stackrel{\circ}{\tau}$, tais que $\beta_{i}^{j}>0(i=0,1,2 ; j=1,2)$ e $\beta_{0}^{j}+\beta_{1}^{j}+\beta_{2}^{j}=1(j=1,2)$. Consideremos ainda o ponto $x=\alpha_{0} v_{0}+\alpha_{1} v_{1}+\alpha_{2} v_{2}+\alpha_{3} v_{3}+\alpha_{4} v_{4}+\alpha_{5} v_{5}+$ $\alpha_{6} v_{6}$, tal que $\alpha_{0}, \alpha_{1}, \ldots, \alpha_{6} \geq 0$, com os coeficientes satisfazendo as seguintes condições:

$$
\left\{\begin{array}{l}
\alpha_{0}+\alpha_{1}+\alpha_{2}=\beta_{0}^{1} \\
\alpha_{3}+\alpha_{4}=\beta_{1}^{1} \\
\alpha_{5}+\alpha_{6}=\beta_{2}^{1}
\end{array}\right.
$$

A aplicação $h_{\sigma}: f^{-1}\left(y^{1}\right) \cap \sigma \longrightarrow f^{-1}\left(y^{2}\right) \cap \sigma$ é definida por

$$
h_{\sigma}(x)=\frac{\beta_{0}^{2}}{\beta_{0}^{1}} \sum_{i=0}^{2} \alpha_{i} v_{i}+\frac{\beta_{1}^{2}}{\beta_{1}^{1}} \sum_{i=3}^{4} \alpha_{i} v_{i}+\frac{\beta_{2}^{2}}{\beta_{2}^{1}} \sum_{i=5}^{6} \alpha_{i} v_{i} .
$$

A aplicação $\left.f\right|_{\theta}: \theta \longrightarrow \tau$ é definida por:

$$
\left\{\begin{array}{l}
f\left(v_{1}\right)=w_{0} \\
f\left(v_{3}\right)=f\left(v_{4}\right)=w_{1} \\
f\left(v_{6}\right)=w_{2}
\end{array}\right.
$$

Se $z=\delta_{1} v_{1}+\delta_{3} v_{3}+\delta_{4} v_{4}+\delta_{6} v_{6}=0 v_{0}+\delta_{1} v_{1}+0 v_{2}+\delta_{3} v_{3}+\delta_{4} v_{4}+0 v_{5}+\delta_{6} v_{6}$, então a aplicação $h_{\theta}: f^{-1}\left(y^{1}\right) \cap \theta \longrightarrow f^{-1}\left(y^{2}\right) \cap \theta$ é definida por

$$
h_{\theta}(z)=0 v_{0}+\frac{\beta_{0}^{2}}{\beta_{0}^{1}} \delta_{1} v_{1}+0 v_{2}+\frac{\beta_{1}^{2}}{\beta_{1}^{1}} \delta_{3} v_{3}+\frac{\beta_{1}^{2}}{\beta_{1}^{1}} \delta_{4} v_{4}+0 v_{5}+\frac{\beta_{2}^{2}}{\beta_{2}^{1}} \delta_{6} v_{6} .
$$

Proposição 2.3.5 Sejam $K$ um complexo simplicial, $\tau$ um n-simplexo, $f: K \rightarrow \tau$ uma aplicação simplicial sobrejetora e $y^{1}, y^{2} \in \stackrel{\circ}{\tau}$. Então $f^{-1}\left(y^{1}\right)$ é homeomorfa à $f^{-1}\left(y^{2}\right)$. 


\section{Demonstração:}

$\mathrm{O}$ complexo $K$ é formado por dois tipos de simplexos. O primeiro tipo é da forma $\sigma$, onde $\left.f\right|_{\sigma}$ é sobrejetora, e esses simplexos serão denominados do tipo 1. O segundo é da forma $\rho$, onde $\left.f\right|_{\rho}$ não é sobrejetora, e esses simplexos serão denominados do tipo 2.

Observe que se $\sigma$ for do tipo 1 , então vale que $f^{-1}\left(y^{1}\right) \cap \sigma \neq \emptyset$ e também $f^{-1}\left(y^{2}\right) \cap \sigma \neq \emptyset$, pois $\left.f\right|_{\sigma}$ é sobrejetora. Por outro lado, se $\rho$ é do tipo 2, então $f^{-1}\left(y^{1}\right) \cap \rho=f^{-1}\left(y^{2}\right) \cap \rho=\emptyset$, senão, pelo Lema $2.3 .2,\left.f\right|_{\rho}$ seria sobrejetora.

Então temos que,

$$
f^{-1}\left(y^{1}\right)=\bigcup_{\substack{\sigma \in K \\ \sigma \text { do tipo 1 }}}\left(f^{-1}\left(y^{1}\right) \cap \sigma\right)
$$

$$
f^{-1}\left(y^{2}\right)=\bigcup_{\substack{\sigma \in K \\ \sigma \text { do tipo } 1}}\left(f^{-1}\left(y^{2}\right) \cap \sigma\right)
$$

Consideremos a aplicação $H_{y^{1}}^{y^{2}}: f^{-1}\left(y^{1}\right) \rightarrow f^{-1}\left(y^{2}\right)$ definida por $H_{y^{1}}^{y^{2}}(x)=$ $h_{\sigma}(x), x \in f^{-1}\left(y^{1}\right) \cap \sigma, \sigma$ do tipo 1 , onde $h_{\sigma}$ é dada logo acima do Lema 2.3.3.

A aplicação $H_{y^{1}}^{y^{2}}$ está bem definida e é um homeomorfismo. De fato, suponha $x \in f^{-1}\left(y^{1}\right) \cap \sigma_{1} \cap \sigma_{2}$, com $\sigma_{1}, \sigma_{2} \in K$. Pelo Lema 2.3.3, temos que

$$
\left.h_{\sigma_{1}}\right|_{f^{-1}\left(y^{1}\right) \cap \sigma_{1} \cap \sigma_{2}}=h_{\sigma_{1} \cap \sigma_{2}}=\left.h_{\sigma_{2}}\right|_{f^{-1}\left(y^{1}\right) \cap \sigma_{1} \cap \sigma_{2}},
$$

então

$$
h_{\sigma_{1}}(x)=h_{\sigma_{1} \cap \sigma_{2}}(x)=h_{\sigma_{2}}(x)
$$

e $H_{y^{1}}^{y^{2}}$ está bem definida.

Vamos verificar agora que a aplicação $H_{y^{1}}^{y^{2}}$ é contínua. Primeiramente observemos que, como $f$ é contínua, $f^{-1}\left(y^{1}\right)$ é um subconjunto fechado em $K$. Como $\left.H_{y^{1}}^{y^{2}}\right|_{f^{-1}\left(y^{1}\right) \cap \sigma}=h_{\sigma}$, pelo Corolário 2.2 .9 segue que $\left.H_{y^{1}}^{y^{2}}\right|_{f^{-1}\left(y^{1}\right) \cap \sigma}$ é contínua, $\forall \sigma \in K$. E pelo Lema 1.1.14, obtemos a continuidade de $H_{y^{1}}^{y^{2}}$. 
Analogamente, obtemos uma aplicação contínua $\bar{H}_{y^{2}}^{y^{1}}: f^{-1}\left(y^{2}\right) \rightarrow f^{-1}\left(y^{1}\right)$ definida por $\bar{H}_{y^{2}}^{y^{1}}(x)=h_{\sigma}^{-1}(x)$, para $x \in f^{-1}\left(y^{2}\right) \cap \sigma$, onde $h_{\sigma}^{-1}: f^{-1}\left(y^{2}\right) \cap \sigma \rightarrow$ $f^{-1}\left(y^{1}\right) \cap \sigma$ é a inversa de $h_{\sigma}: f^{-1}\left(y^{1}\right) \cap \sigma \rightarrow f^{-1}\left(y^{2}\right) \cap \sigma$, sendo $h_{\sigma}$ dado pelo Corolário 2.2.9.

Para concluir a demonstração, basta verificar que $\bar{H}_{y^{2}}^{y^{1}}$ é a inversa de $H_{y^{1}}^{y^{2}}$. Seja $x \in f^{-1}\left(y^{1}\right) \cap \sigma$, para algum $\sigma \in K$, então temos que

$$
\bar{H}_{y^{2}}^{y^{1}}\left(H_{y^{1}}^{y^{2}}(x)\right)=\bar{H}_{y^{2}}^{y^{1}}\left(h_{\sigma}(x)\right)=h_{\sigma}^{-1}\left(h_{\sigma}(x)\right)=x
$$

e, se $z \in f^{-1}\left(y^{2}\right) \cap \sigma$, para algum $\sigma \in K$, então

$$
H_{y^{1}}^{y^{2}}\left(\bar{H}_{y^{2}}^{y^{1}}(z)\right)=H_{y^{1}}^{y^{2}}\left(h_{\sigma}^{-1}(z)\right)=h_{\sigma}\left(h_{\sigma}^{-1}(z)\right)=z .
$$

Portanto, $H_{y^{1}}^{y^{2}}$ é um homeomorfismo entre $f^{-1}\left(y^{1}\right)$ e $f^{-1}\left(y^{2}\right)$.

Observação 2.3.6 As fibras $f^{-1}\left(y^{1}\right)$ e $f^{-1}\left(y^{2}\right)$ têm o mesmo número de componentes conexas.

No lema abaixo, utilizamos a notação formal para espaços subjacentes.

Lema 2.3.7 Seja $f: K \rightarrow L$ uma aplicação simplicial entre complexos simpliciais. Se para $\sigma \in K, \tau \in L$, existir $x \in \stackrel{\circ}{\sigma}$ tal que $|f|(x) \in \stackrel{\circ}{\tau}$, então $|f|(\sigma)=\tau$ e também $f(\sigma)=\tau$.

\section{Demonstração:}

Como $f$ é simplicial, $f(\sigma)=\tilde{\tau}$ é um simplexo de $L$. Além disso, temos que ter $\tau<\tilde{\tau}$ pela definição de $|f|$. Suponha que $\tau \ll \tilde{\tau}$. Como $|f|: \sigma \rightarrow \tilde{\tau}$ é sobrejetora, pelo Lema 2.3.1, temos que $|f|^{-1}(\tau) \ll \sigma$, o qual implica que $|f|^{-1}(\tau) \cap \stackrel{\circ}{\sigma}=\emptyset$. Isto é uma contradição. Por isso, temos que $\tau=\tilde{\tau}$. 
Lema 2.3.8 Sejam $K, L$ complexos simpliciais e $f: K \rightarrow L$ uma aplicação simplicial. Consideremos ainda $\tau \in L$ um simplexo de $L$, então $f^{-1}(\tau)$ é um subcomplexo de $K$.

\section{Demonstração:}

Seja $x \in f^{-1}(\tau) \subset K$, então existe um $\sigma \in K$ tal que $x \in \stackrel{\circ}{\sigma}$. Consideremos agora $\theta<\tau$ tal que $f(x) \in \stackrel{\circ}{\theta}$. Pelo Lema 2.3.7, $f(\sigma)=\theta \subset \tau$ e isso mostra que $\sigma \subset f^{-1}(\tau)$ e que $f^{-1}(\tau)$ é uma coleção de simplexos. Se $\sigma \in f^{-1}(\tau)$, claramente, qualquer face de $\sigma$ também pertence a $f^{-1}(\tau)$.

Consideremos agora $\sigma_{1}$ e $\sigma_{2}$ dois simplexos de $f^{-1}(\tau)$. Como $f^{-1}(\tau)$ é um subconjunto de $K$, temos que $\sigma_{1}, \sigma_{2} \in K$ e como $K$ é um complexo simplicial, segue que $\sigma_{1} \cap \sigma_{2}$ é uma face comum a $\sigma_{1}$ e a $\sigma_{2}$. Com isso mostramos que $f^{-1}(\tau)$ é um complexo simplicial, ou seja, um subcomplexo de $K$.

A proposição abaixo, provada com as técnicas aqui desenvolvidas, é fundamental nos capítulos subseqüentes. Uma outra demonstração pode ser encontrada em [MS, Lemma 20.5].

Proposição 2.3.9 Sejam $K, L$ complexos simpliciais, $f: K \rightarrow L$ uma aplicação simplicial, $\tau \subset f(K)$ um simplexo de $L$ e $b_{\tau}$ o baricentro de $\tau$. Então existe um homeomorfismo entre espaços topológicos $\Omega_{\tau}: f^{-1}(\stackrel{\circ}{\tau}) \rightarrow f^{-1}\left(b_{\tau}\right) \times \stackrel{\circ}{\tau}$ que faz o seguinte diagrama comutar:

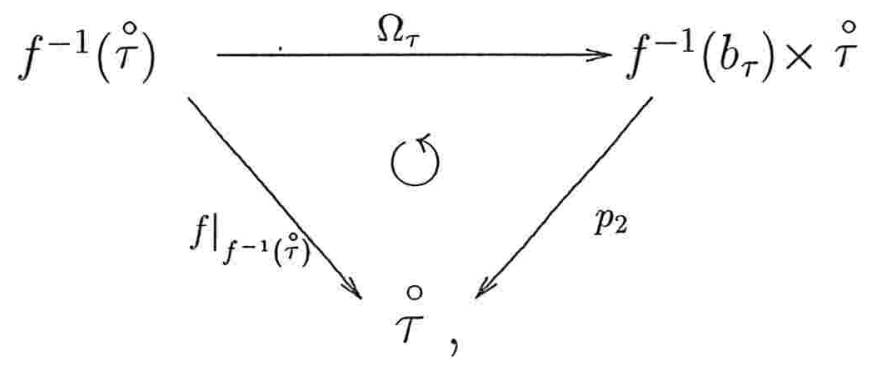

com $p_{2}(a, c)=c$, para $\forall(a, c) \in f^{-1}\left(b_{\tau}\right) \times \stackrel{\circ}{\tau}$. 


\section{Demonstração:}

Seja $x \in f^{-1}(\stackrel{\circ}{\tau})$; pelo Lema 2.3 .8 temos que $f^{-1}(\tau)$ é um complexo simplicial, portanto aplicando a Proposição 2.3 .5 para $\left.f\right|_{f^{-1}(\tau)}: f^{-1}(\tau) \rightarrow \tau$ e os pontos $y^{1}=f(x)$ e $y^{2}=b_{\tau}$, obtemos o homeomorfismo entre as fibras $H_{f(x)}^{b_{\tau}}: f^{-1}(f(x)) \rightarrow f^{-1}\left(b_{\tau}\right)$, que será denotado simplesmente por $H_{f(x)}$.

Se $w_{0}, w_{1}, \ldots, w_{n}$ são os vértices de $\tau$ e $v_{0}, v_{1}, \ldots, v_{m}$ são os vértices de um simplexo $\theta$ de $f^{-1}(\tau)$ tal que $x \in \theta$, então com as mesmas notações do Lema 2.3.3, temos que:

$$
H_{f(x)}(x)=h_{\theta}(x)=\sum_{i=0}^{i_{0}}\left(\frac{1}{\beta_{0}(n+1)} \alpha_{i} v_{i}\right)+\cdots+\sum_{i=i_{n-1}+1}^{i_{n}}\left(\frac{1}{\beta_{n}(n+1)} \alpha_{i} v_{i}\right) \text {, }
$$

onde $f(x)=\sum_{k=0}^{n} \beta_{k} w_{k}, \beta_{k}>0, \sum_{k=0}^{n} \beta_{k}=1, x=\sum_{i=0}^{m} \alpha_{i} v_{i}, \alpha_{i}>0, \sum_{i=i_{k-1}+1}^{i_{k}} \alpha_{i}=$ $\beta_{k}$ e $b_{\tau}=\sum_{k=0}^{n} \frac{1}{n+1} w_{k}$

\section{Portanto}

$H_{f(x)}(x)=\frac{1}{n+1}\left(\frac{\alpha_{0} v_{0}+\cdots+\alpha_{i_{0}} v_{i_{0}}}{\alpha_{0}+\cdots+\alpha_{i_{0}}}+\cdots+\frac{\alpha_{i_{n-1}+1} v_{i_{n-1}+1}+\cdots+\alpha_{i_{n}} v_{i_{n}}}{\alpha_{i_{n-1}+1}+\cdots+\alpha_{i_{n}}}\right)$

é uma aplicação contínua para $x \in f^{-1}(\stackrel{\circ}{\tau}) \cap \theta$.

Definindo agora a aplicação $\Omega_{\tau}: f^{-1}(\stackrel{\circ}{\tau}) \rightarrow f^{-1}\left(b_{\tau}\right) \times \stackrel{\circ}{\tau}$ como sendo $\Omega_{\tau}(x)=\left(H_{f(x)}(x), f(x)\right)$, ela está bem definida, pois $H$ e $f$ também o estão, $H_{f(x)}(x) \in f^{-1}\left(b_{\tau}\right)$ e $f(x) \in \stackrel{\circ}{\tau}$, para qualquer $x \in f^{-1}(\stackrel{\circ}{\tau})$. Como $H_{f(x)}(x)$ é contínua para $x \in f^{-1}(\stackrel{\circ}{\tau}) \cap \theta$, segue que $\left.\Omega_{\tau}\right|_{f^{-1}(\stackrel{\circ}{\tau}) \cap \theta}$ é contínua para qualquer $\theta$ tal que $f^{-1}(\stackrel{\circ}{\tau}) \cap \theta \neq \emptyset$. Então, pelo Lema 1.1.14, temos que $\Omega_{\tau}$ é contínua (note que $f^{-1}(\stackrel{\circ}{\tau})$ é aberto de $f^{-1}(\tau)$, e por isso podemos usar o item (2) do Lema 1.1.14).

Consideremos agora a aplicação $\bar{\Omega}_{\tau}: f^{-1}\left(b_{\tau}\right) \times \stackrel{\circ}{\tau} \rightarrow f^{-1}(\stackrel{\circ}{\tau})$ definida por $\bar{\Omega}_{\tau}\left(z, y^{1}\right)=\bar{H}_{b_{\tau}}^{y^{1}}(z):=\bar{H}^{y^{1}}(z)$, para cada $\left(z, y^{1}\right) \in f^{-1}\left(b_{\tau}\right) \times \stackrel{\circ}{\tau}$, onde $\bar{H}_{b_{\tau}}^{y^{1}}$ está 
definida na demonstração da Proposição 2.3.5, ou seja,

$$
\bar{\Omega}_{\tau}\left(z, y^{1}\right)=(n+1)\left(\beta_{0} \sum_{i=0}^{i_{0}} \delta_{i} u_{i}+\cdots+\beta_{n} \sum_{i=i_{n-1}+1}^{i_{n}} \delta_{i} u_{i}\right),
$$

onde $y^{1}=\sum_{k=0}^{n} \beta_{k} w_{k}, \beta_{k}>0, \sum_{k=0}^{n} \beta_{k}=1, b_{\tau}=\sum_{k=0}^{n} \frac{1}{n+1} w_{k}, u_{i}(i=0, \ldots, m)$ são vértices tais que $z \in\left\langle u_{0} \cdots u_{m}\right\rangle \in K, z=\sum_{i=0}^{m} \delta_{i} u_{i}, \delta_{i} \geq 0$ e $\sum_{i=i_{k-1}+1}^{i_{k}} \delta_{i}=\frac{1}{n+1}$. Temos também que $\bar{\Omega}_{\tau}$ está bem definida. Seguindo os passos da demonstração da continuidade de $\Omega_{\tau}$ e utilizando o [Mu, Theorem 20.4, p.115], mostramos a continuidade de $\bar{\Omega}_{\tau}$.

Agora, basta verificar que $\bar{\Omega}_{\tau}$ é a inversa de $\Omega_{\tau}$.

Seja $\left(z, y^{1}\right) \in f^{-1}\left(b_{\tau}\right) \times \stackrel{\circ}{\tau}$, então

$$
\begin{aligned}
\Omega_{\tau}\left(\bar{\Omega}_{\tau}\left(z, y^{1}\right)\right) & =\Omega_{\tau}\left(\bar{H}^{y^{1}}(z)\right) \\
& =\left(H_{f\left(\bar{H}^{y^{1}}(z)\right)}\left(\bar{H}^{y^{1}}(z)\right), f\left(\bar{H}^{y^{1}}(z)\right)\right) \\
& =\left(H_{y^{1}}\left(\bar{H}^{y^{1}}(z)\right), y^{1}\right) \\
& =\left(z, y^{1}\right) .
\end{aligned}
$$

Consideremos agora $x \in f^{-1}(\stackrel{\circ}{\tau})$, temos que

$$
\begin{aligned}
\bar{\Omega}_{\tau}\left(\Omega_{\tau}(x)\right) & =\bar{\Omega}_{\tau}\left(H_{f(x)}(x), f(x)\right) \\
& =\bar{H}^{f(x)}\left(H_{f(x)}(x)\right) \\
& =x .
\end{aligned}
$$

Portanto, $\Omega_{\tau}$ é um homeomorfismo entre $f^{-1}(\stackrel{\circ}{\tau})$ e $f^{-1}\left(b_{\tau}\right) \times \stackrel{\circ}{\tau}$, ainda mais, $p_{2}\left(\Omega_{\tau}(x)\right)=p_{2}\left(H_{f(x)}(x), f(x)\right)=f(x), \forall x \in f^{-1}(\stackrel{\circ}{\tau})$, ou seja, $p_{2} \circ \Omega_{\tau}=\left.f\right|_{f^{-1}(\tau)}$. 


\subsection{Lemas sobre imagens inversas de simple- xos}

Sejam $f: K \rightarrow L$ uma aplicação simplicial, $\tau<\eta$ dois simplexos de $f(K)$. Nesta seção mostramos que o fibrado sobre o interior de $\tau$ é um retrato por deformação do fibrado sobre $\stackrel{\circ}{\eta} \cup \stackrel{\circ}{\tau}$. Este resultado é utilizado no próximo capítulo para mostrar que a fatorização de Stein de uma aplicação simplicial é um poliedro.

Lema 2.4.1 Sejam $\theta, \eta$ dois simplexos de dimensões $m, n$, respectivamente, e $f: \theta \rightarrow \eta$ uma aplicação simplicial e sobrejetora. Se $\tau<\eta$ é uma face de $\eta$, então $f^{-1}(\stackrel{\circ}{\tau})$ é um retrato forte de deformação de $f^{-1}(\stackrel{\circ}{\eta} \cup \stackrel{\circ}{\tau})$.

\section{Demonstração:}

Sejam $v_{0}, v_{1}, \ldots, v_{m}$ os vértices de $\theta ; w_{0}, w_{1}, \ldots, w_{n}$ os vértices de $\eta \mathrm{e}$ $f: \theta \rightarrow \eta$ dada por:

$$
\begin{gathered}
f\left(v_{0}\right)=\cdots=f\left(v_{i_{0}}\right)=w_{0}, \\
f\left(v_{i_{0}+1}\right)=\cdots=f\left(v_{i_{1}}\right)=w_{1} \\
\vdots \\
f\left(v_{i_{n-1}+1}\right)=\cdots=f\left(v_{i_{n}}\right)=w_{n} .
\end{gathered}
$$

Reordenando os índices dos vértices de $\eta$, se necessário, temos que os vértices de $\tau$ são $w_{0}, \ldots, w_{k}$.

Denotemos por $F$ a face $f^{-1}(\tau)$ de $\theta$, formada pelos vértices $v_{0}, \ldots, v_{i_{k}}$ e consideremos a face $\rho<\theta$ de vértices $v_{i_{k}+1}, \ldots, v_{i_{n}}$. Pela Proposição 2.1.3, temos que $\rho, F$ são conjuntos independentes, ou seja, dado $x \in \rho F=\theta, x$ se escreve de forma única como sendo $x=\lambda_{x} \alpha_{x}+\mu_{x} \beta_{x}$, com $\alpha_{x} \in \rho, \beta_{x} \in F$, $\lambda_{x}, \mu_{x} \geq 0$ e $\lambda_{x}+\mu_{x}=1$.

Vamos verificar que $\Psi: f^{-1}(\stackrel{\circ}{\eta} \cup \stackrel{\circ}{\tau}) \longrightarrow f^{-1}(\stackrel{\circ}{\tau})$ definida por $\Psi(x)=\beta_{x}$ está bem definida. Observemos que $x \notin \rho$, pois se $x \in \rho$, então $x$ seria da 
forma $\lambda_{x} \alpha_{x}, \operatorname{com} \alpha_{x}=\alpha_{i_{k}+1} v_{i_{k}+1}+\cdots+\alpha_{i_{n}} v_{i_{n}}$, e $f(x)=\sum_{j=k+1}^{n} \delta_{j} w_{j} \notin \stackrel{\circ}{\eta}$ $\cup \stackrel{\circ}{\tau}$ para alguns $\delta_{j}$ implica que $x \notin f^{-1}(\stackrel{\circ}{\eta} \cup \stackrel{\circ}{\tau})$, o que é uma contradição. Portanto, $x \notin \rho$ e existe um único $\beta_{x} \in F$ tal que $x=\lambda_{x} \alpha_{x}+\mu_{x} \beta_{x}\left(\mu_{x}>0\right)$.

Para concluir que $\Psi$ está bem definida, resta-nos provar que $\beta_{x} \in f^{-1}(\stackrel{\circ}{\tau})$. Observemos que se $x \in f^{-1}(\stackrel{\circ}{\tau})$, então $\Psi(x)=x$ e $\Psi(x) \in f^{-1}(\stackrel{\circ}{\tau})$. Portanto, supondo $x \in f^{-1}(\stackrel{\circ}{\eta})$ e que $\Psi(x) \notin f^{-1}(\stackrel{\circ}{\tau})$, temos $\Psi(x) \in F \backslash f^{-1}(\stackrel{\circ}{\tau})$. Então $f(\Psi(x)) \in \tau \backslash \stackrel{\circ}{\tau}$ e disto segue que $f(\Psi(x))$ pertence a $\delta$, uma face de $\tau$ de dimensão $l, \operatorname{com} l<k$.

Seja $x$ pertencente ao segmento de extremidades $\alpha_{x}$ e $\beta_{x}$, com $\alpha_{x} \in \rho$ e $\beta_{x}=\Psi(x) \in F$. Como $f$ é linear, temos que $f(x)$ pertence ao segmento de extremidades $f\left(\alpha_{x}\right) \in \zeta$ e $f\left(\beta_{x}\right) \in \delta$, onde $\zeta=\left\langle w_{k+1} \cdots w_{n}\right\rangle$. Então $f(x)$ pertence a uma face própria de $\eta$, ou seja, $f(x) \notin \stackrel{\circ}{\eta}$ e $x \notin f^{-1}(\stackrel{\circ}{\eta})$, o que é uma contradição. Portanto, $\Psi(x) \in f^{-1}(\stackrel{\circ}{\tau})$ e $\Psi$ está bem definida.

Já observamos acima que $\left.\Psi\right|_{f^{-1}(\tau)}$ é igual à aplicação identidade restrita a $f^{-1}(\stackrel{\circ}{\tau})$. Ao verificarmos que $\Psi$ é contínua, estaremos concluindo que $f^{-1}(\stackrel{\circ}{\tau})$ é um retrato de $f^{-1}(\stackrel{\circ}{\eta} \cup \stackrel{\circ}{\tau})$.

Consideremos agora o seguinte diagrama comutativo:

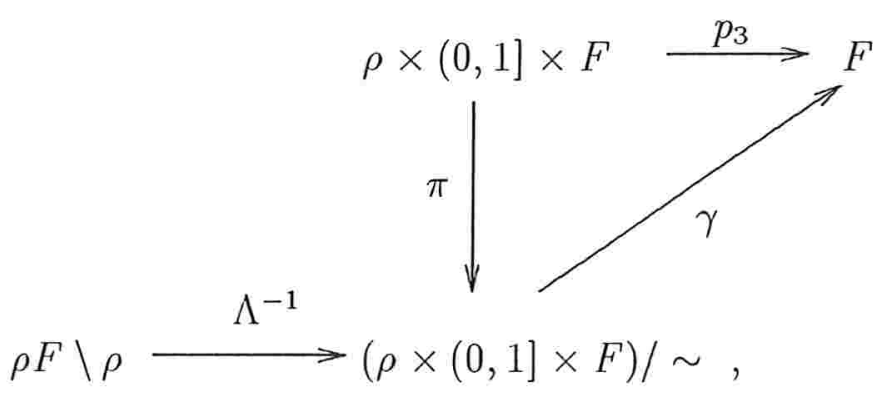


onde $\pi$ é a restrição da projeção definida na demonstração da Proposição 2.1.1; $p_{3}$ é a projeção canônica que associa a cada elemento $(\alpha, \mu, \beta)$ de $\rho \times(0,1] \times F$ o elemento $\beta \in F$ e $\Lambda^{-1}$ é a inversa do homeomorfismo dado na demonstração do Corolário 2.1 .2 trocando $P$ por $\rho$ e $Q$ por $F$. Finalmente, definamos $\gamma:(\rho \times(0,1] \times F) / \sim \rightarrow F$ como sendo a aplicação que associa a cada classe de equivalência $[\alpha, \mu, \beta]$ o elemento $\beta$ de $F$.

A aplicação $\gamma$ está bem definida, pois considerando dois representantes diferentes $[\alpha, \mu, \beta]$ e $\left[\alpha^{\prime}, \mu^{\prime}, \beta^{\prime}\right]$ de uma mesma classe de $(\rho \times(0,1] \times F) / \sim$ temos que:

1) se $\mu \in(0,1)$, então $\alpha=\alpha^{\prime}, \beta=\beta^{\prime}, \mu=\mu^{\prime}$ e $\gamma([\alpha, \mu, \beta])=\beta=\beta^{\prime}=$ $\gamma\left(\left[\alpha^{\prime}, \mu^{\prime}, \beta^{\prime}\right]\right)$,

2) se $\mu=1$, então $\mu^{\prime}=1, \beta=\beta^{\prime}$ e $\gamma([\alpha, \mu, \beta])=\beta=\beta^{\prime}=\gamma\left(\left[\alpha^{\prime}, \mu^{\prime}, \beta^{\prime}\right]\right)$.

Mais ainda, considerando a topologia quociente em $(\rho \times(0,1] \times F) / \sim$, segue que $\gamma$ é contínua, pois a projeção $p_{3}$ também é contínua.

Consideremos a aplicação $\Gamma: \rho F \backslash \rho \longrightarrow F$ definida como sendo a composta $\gamma \circ \Lambda^{-1}$. Pela demonstração do Corolário 2.1.2, sabemos que $\Lambda^{-1}$ é contínua, portanto segue que $\Gamma$ também é contínua.

Observemos ainda que $\Psi=\left.\Gamma\right|_{f^{-1}\left(\dot{\circ} \cup \tau^{\circ}\right)}$, pois já vimos que $f^{-1}(\stackrel{\circ}{\eta} \cup \stackrel{\circ}{\tau}) \subset$ $\rho F \backslash \rho$ e temos que $f^{-1}(\stackrel{\circ}{\tau}) \subset f^{-1}(\tau)=F$. Ainda mais, se $x \in f^{-1}(\stackrel{\circ}{\eta} \cup \stackrel{\circ}{\tau})$, então $\Gamma(x)=\gamma \circ \Lambda^{-1}\left(\left(1-\mu_{x}\right) \alpha_{x}+\mu_{x} \beta_{x}\right)=\gamma\left(\left[\alpha_{x}, \mu_{x}, \beta_{x}\right]\right)=\beta_{x}=\Psi(x)$, $\forall x \in f^{-1}(\stackrel{\circ}{\eta} \cup \stackrel{\circ}{\tau})$. Como a restrição de uma aplicação contínua ainda é uma aplicação contínua, segue que $\Psi$ é contínua. Concluímos assim que $f^{-1}(\stackrel{\circ}{\tau})$ é um retrato de $f^{-1}(\stackrel{\circ}{\eta} \cup \stackrel{\circ}{\tau})$. 
Finalmente, definimos $H: f^{-1}(\stackrel{\circ}{\eta} \cup \stackrel{\circ}{\tau}) \times[0,1] \longrightarrow f^{-1}(\stackrel{\circ}{\eta} \cup \stackrel{\circ}{\tau})$ da seguinte forma: para cada $x \in f^{-1}(\stackrel{\circ}{\eta} \cup \stackrel{\circ}{\tau})$ e $t \in[0,1]$, seja $H(x, t)=t \Psi(x)+(1-t) x$. Como $x$ e $\Psi(x)$ pertencem ao simplexo $\theta$, temos que $t \Psi(x)+(1-t) x$ faz sentido, é um elemento de $\sigma$ e é de $f^{-1}(\stackrel{\circ}{\tau})$. Conseqüentemente a aplicação $H$ está bem definida. Além disso, $H$ é contínua, pois $\Psi$ é contínua e $H$ é produto e soma de aplicações contínuas. Temos ainda que valem: $H(x, 0)=$ $x, \forall x \in f^{-1}(\stackrel{\circ}{\eta} \cup \stackrel{\circ}{\tau}), H(x, 1)=\Psi(x) \in f^{-1}(\stackrel{\circ}{\tau}), \forall x \in f^{-1}(\stackrel{\circ}{\eta} \cup \stackrel{\circ}{\tau})$ e $H(a, t)=$ $a, \forall a \in f^{-1}(\stackrel{\circ}{\tau}), \forall t \in[0,1]$, ou seja, $H$ é uma homotopia entre a retração $\Psi$ e a aplicação identidade id $: f^{-1}(\stackrel{\circ}{\eta} \cup \stackrel{\circ}{\tau}) \rightarrow f^{-1}(\stackrel{\circ}{\eta} \cup \stackrel{\circ}{\tau})$. Portanto, $f^{-1}(\stackrel{\circ}{\tau})$ é um retrato forte de deformação de $f^{-1}(\stackrel{\circ}{\eta} \cup \stackrel{\circ}{\tau})$.

Lema 2.4.2 Sejam $K, L$ dois complexos simpliciais e $f: K \rightarrow L$ uma aplicaf̧ão simplicial sobrejetora. Suponhamos que $K$ é localmente finito. Se $\tau<\eta$ são simplexos de $L$, então $f^{-1}(\stackrel{\circ}{\tau})$ é um retrato forte de deformação de $f^{-1}(\stackrel{\circ}{\eta} \cup \stackrel{\circ}{\tau})$

\section{Demonstração:}

Consideremos os simplexos: $\eta=\left\langle w_{0} w_{1} \cdots w_{n}\right\rangle \in L, \tau=\left\langle w_{0} \cdots w_{k}\right\rangle$ uma face própria de $\eta$ e $\theta=\left\langle v_{0} v_{1} \cdots v_{m}\right\rangle \in K$ tal que $f^{-1}(\stackrel{\circ}{\eta} \cup \stackrel{\circ}{\tau}) \cap \stackrel{\circ}{\theta} \neq \emptyset$.

Se $f^{-1}(\stackrel{\circ}{\eta}) \cap \stackrel{\circ}{\theta}=\emptyset$, então $f^{-1}(\stackrel{\circ}{\tau}) \cap \stackrel{\circ}{\theta} \neq \emptyset$ e, pelo Lema 2.3.7, $\left.f\right|_{\theta}: \theta \rightarrow \tau$ é sobrejetora e $\Psi_{\theta}: f^{-1}(\stackrel{\circ}{\eta} \cup \stackrel{\circ}{\tau}) \cap \theta=f^{-1}(\stackrel{\circ}{\tau}) \cap \theta \rightarrow f^{-1}(\stackrel{\circ}{\tau}) \cap \theta$, que é a aplicação definida na demonstração do Lema 2.4 .1 restrita ao simplexo $\theta$, é a própria aplicação identidade e nada temos a fazer. Portanto, suponha $f^{-1}(\stackrel{\circ}{\eta}) \cap \stackrel{\circ}{\theta} \neq \emptyset$.

Reordenando, eventualmente, os índices dos vértices de $\theta$ e $\eta$ temos que $f$ é definida por:

$$
\begin{gathered}
f\left(v_{0}\right)=\cdots=f\left(v_{i_{0}}\right)=w_{0} \\
f\left(v_{i_{0}+1}\right)=\cdots=f\left(v_{i_{1}}\right)=w_{1}, \\
\vdots \\
f\left(v_{i_{n-1}+1}\right)=\cdots=f\left(v_{i_{n}}\right)=w_{n} .
\end{gathered}
$$


Para mostrar que $f^{-1}(\stackrel{\circ}{\tau})$ é um retrato de $f^{-1}(\stackrel{\circ}{\eta} \cup \stackrel{\circ}{\tau})$ basta verificarmos que, se $\nu$ é uma face qualquer de $\theta$, então $\Psi_{\nu}=\left.\Psi_{\theta}\right|_{f^{-1}(\dot{\eta} \cup \tau) \cap}$, , onde

$$
\Psi_{\theta}: f^{-1}(\stackrel{\circ}{\eta} \cup \stackrel{\circ}{\tau}) \cap \theta \longrightarrow f^{-1}(\stackrel{\circ}{\tau}) \cap \theta
$$

é a aplicação $\Psi$ definida na demonstração do Lema 2.4 .1 restrita ao simplexo $\theta$, e

$$
\Psi_{\nu}: f^{-1}(\stackrel{\circ}{\eta} \cup \stackrel{\circ}{\tau}) \cap \nu \longrightarrow f^{-1}(\stackrel{\circ}{\tau}) \cap \nu
$$

é a aplicação $\Psi$ definida na demonstração do Lema 2.4.1 restrita ao simplexo $\nu$.

Primeiramente, observemos que se $\nu$ satisfaz $f^{-1}(\stackrel{\circ}{\eta} \cup \stackrel{\circ}{\tau}) \cap \nu=\emptyset$, então nada temos a fazer. Consideremos, portanto, uma face $\nu$ de $\theta$ tal que $f^{-1}(\stackrel{\circ}{\eta} \cup \stackrel{\circ}{\tau}) \cap \nu \neq \emptyset$. Pelo mesmo argumento, podemos supor $f^{-1}(\stackrel{\circ}{\eta}) \cap \nu \neq \emptyset \mathrm{e}$ aplicando o Lema 2.4 .1 para $\left.f\right|_{\nu}: \nu \longrightarrow \eta$, temos que $f^{-1}(\tau) \cap \nu$ é um retrato forte de deformação de $f^{-1}(\stackrel{\circ}{\eta} \cup \stackrel{\circ}{\tau}) \cap \nu$.

Seja $\Psi_{\nu}: f^{-1}(\stackrel{\circ}{\eta} \cup \stackrel{\circ}{\tau}) \cap \nu \longrightarrow f^{-1}(\stackrel{\circ}{\tau}) \cap \nu$ definida por $\Psi_{\nu}\left(\lambda_{x} \alpha_{x}+\mu_{x} \beta_{x}\right)=\beta_{x}$, onde $\lambda_{x}, \mu_{x} \geq 0, \lambda_{x}+\mu_{x}=1, \alpha_{x} \in \rho_{\nu}$, e $\rho_{\nu}$ é a face de $\nu$ gerada por todos os vértices $v_{i} \in\left(\theta \backslash f^{-1}(\tau)\right) \cap \nu \subset \theta \backslash f^{-1}(\tau)$ e $\beta_{x} \in f^{-1}(\stackrel{\circ}{\tau}) \cap \nu \subset f^{-1}(\stackrel{\circ}{\tau}) \cap \theta$. Claramente, pela unicidade de $\beta_{x}$, temos que $\Psi_{\nu}=\left.\Psi_{\theta}\right|_{f^{-1}(\dot{\circ} \cup \tau) \cap \nu}$.

Consideremos agora a aplicação $\Psi: f^{-1}(\stackrel{\circ}{\eta} \cup \stackrel{\circ}{\tau}) \longrightarrow f^{-1}(\stackrel{\circ}{\tau})$ definida por $\Psi(x)=\Psi_{\theta}(x)$, onde $x \in \theta$, para algum $\theta \in K$. Note que $f^{-1}(\stackrel{\circ}{\eta} \cup \stackrel{\circ}{\tau})=$ $\bigcup_{\theta \in K}\left(f^{-1}(\stackrel{\circ}{\eta} \cup \stackrel{\circ}{\tau}) \cap \theta\right)$.

A aplicação $\Psi$ está bem definida, pois se $x \in f^{-1}(\stackrel{\circ}{\eta} \cup \stackrel{\circ}{\tau}) \cap \nu \cap \theta$, então $\Psi_{\nu}(x)=\Psi_{\theta}(x)$.

Também temos que $\Psi$ é contínua. De fato, $\Psi$ é contínua pelo Lema 1.1.14 e pela construção de $\Psi$ no Lema 2.4 .1 (note que estamos assumindo que $K$ é localmente finito). 
Considere a aplicação $H: f^{-1}(\stackrel{\circ}{\eta} \cup \stackrel{\circ}{\tau}) \times[0,1] \longrightarrow f^{-1}(\stackrel{\circ}{\eta} \cup \stackrel{\circ}{\tau})$ como na demonstração do Lema 2.4.1, ou seja, $H(x, t)=t \Psi(x)+(1-t) x$. Se $x \in \theta$ para algum $\theta \in f^{-1}(\tau) \subset K$, então $x$ e $\Psi(x)$ pertencem ao mesmo simplexo $\theta$. Por isso $t \Psi(x)+(1-t) x$ tem sentido e é um elemento de $\theta$. Também não é difícil mostrar que ele é um elemento de $f^{-1}(\stackrel{\circ}{\tau})$. Conseqüentemente, $H$ está bem definida. E por um lema similar ao Lema 1.1.14, podemos mostrar que $H$ é contínua. Portanto, concluímos que $f^{-1}(\stackrel{\circ}{\tau})$ é um retrato forte de deformação de $f^{-1}(\stackrel{\circ}{\eta} \cup \stackrel{\circ}{\tau})$. 


\section{Capítulo 3}

\section{A fatorização de Stein de uma aplicação simplicial é triangulável}

O objetivo aqui é provar o seguinte resultado: "A fatorização de Stein de qualquer aplicação simplicial própria entre complexos simpliciais localmente finitos é um poliedro." Utilizando este resultado, demonstramos o Teorema 1.3.8.

\subsection{Exemplos}

Embora não seja utilizado no decorrer da tese, citamos abaixo um resultado que ilustra algumas propriedades da fatorização de Stein e que motivam alguns exemplos. O último deles mostra a necessidade de fazermos uma subdivisão baricêntrica na imagem de uma aplicação simplicial, $f: K \rightarrow L$, a fim de obtermos um poliedro homeomorfo à fatorização de Stein de $f$.

Neste capítulo retomamos a notação $|f|:|K| \rightarrow|L|$ para a aplicação entre os subespaços subjacentes de $K$ e $L$, respectivamente. 
Proposição 3.1.1 Seja $f: K \rightarrow L$ uma aplicação simplicial sobrejetora entre dois complexos simpliciais localmente finitos $K$ e $L$, tal que $|f|:|K| \rightarrow|L|$ é própria. Se $|f|^{-1}(y)$ é conexa para qualquer $y \in|L|$, então $W_{|f|}$ é homeomorfo a $|L|$.

\section{Demonstração:}

Como $K$ e $L$ são localmente finitos, $|K|$ e $|L|$ são localmente compactos pelo [Mu, Lemma 2.6, pg.11]. Também $|K|$ e $|L|$ são Hausdorff pelo [Mu, Lemma 2.4, pg.10]. Como a aplicação $|f|$ é própria, então $|f|$ é fechada pela [Br, Prop.11.5, pg.33].

Definimos a aplicação $\zeta:|L| \rightarrow W_{|f|}$ da seguinte forma: para $y \in|L|$, $\zeta(y)=q_{|f|}\left(|f|^{-1}(y)\right)$.

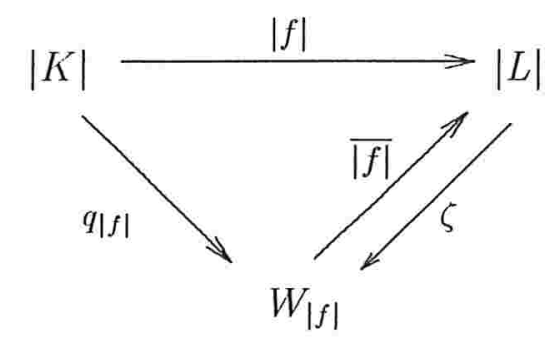

A aplicação $\zeta$ está bem definida, pois $|f|^{-1}(y)$ é conexa e não vazia para cada $y \in|L|$, portanto $q_{|f|}\left(|f|^{-1}(y)\right)$ é um único ponto de $W_{|f|}$.

Verifiquemos agora que $\zeta$ é a inversa de $\overline{|f|}$; de fato:

i) $\overline{|f|} \circ \zeta=\operatorname{id}_{|L|}=$ aplicação identidade de $|L|$.

Seja $y \in|L|$, então

$$
\begin{aligned}
\overline{|f|}(\zeta(y)) & =\overline{|f|}\left(q_{|f|}\left(|f|^{-1}(y)\right)\right) \\
& =\left(\overline{|f|} \circ q_{|f|}\right)\left(|f|^{-1}(y)\right) \\
& =|f|\left(|f|^{-1}(y)\right) \\
& =y
\end{aligned}
$$

onde a igualdade (3.2) vale, pois $|f|$ é sobrejetora. 
ii) $\zeta \circ \overline{|f|}=\mathrm{id}_{W_{|f|}}=$ aplicação identidade de $W_{|f|}$.

Seja $z \in W_{|f|}$, então

$$
\begin{aligned}
\zeta(\overline{|f|}(z)) & =q_{|f|}\left(|f|^{-1}(\overline{|f|}(z))\right) \\
& =z,
\end{aligned}
$$

pois todas as fibras de $|f|$ são conexas.

Basta mostrar agora que $\zeta$ é contínua. De fato, seja $A \subset W_{|f|}$ um conjunto fechado de $W_{|f|}$. Como $q_{|f|}$ é contínua, temos que $q_{|f|}^{-1}(A)$ é um conjunto fechado de $|K|$. Mas $|f|$ é uma aplicação fechada, portanto $|f|\left(q_{|f|}^{-1}(A)\right)$ é um fechado de $|L|$.

Vamos verificar agora que $|f|\left(q_{|f|}^{-1}(A)\right)=\zeta^{-1}(A)$.

(つ) Se $y \in \zeta^{-1}(A)$, então $\zeta(y)=x \in A$. Então temos que $q_{|f|}\left(|f|^{-1}(y)\right)=x$ e isto implica que $|f|^{-1}(y) \subset q_{|f|}^{-1}(x)$. Portanto, $y \in|f|\left(q_{|f|}^{-1}(x)\right)$, pois $|f|$ é sobrejetora, e então $y \in|f|\left(q_{|f|}^{-1}(A)\right)$.

(C) Se $y \in|f|\left(q_{|f|}^{-1}(A)\right)$, então existe $x \in A$ tal que $y \in|f|\left(q_{|f|}^{-1}(x)\right)=\{y\}$ e $|f|^{-1}(y)=|f|^{-1}\left(|f|\left(q_{|f|}^{-1}(x)\right)\right)=q_{|f|}^{-1}(x)$. A última igualdade vale, pois estamos assumindo, novamente, que todas as fibras de $|f|$ são conexas. Portanto, $\zeta(y)=q_{|f|}\left(|f|^{-1}(y)\right)=q_{|f|}\left(q_{|f|}^{-1}(x)\right)=x$, pois $q_{|f|}$ é sobrejetora. Além disso, $x \in A$, ou seja, $y \in \zeta^{-1}(x) \subset \zeta^{-1}(A)$.

Portanto, $\zeta^{-1}(A)$ é um conjunto fechado de $|L|$ e $\zeta$ é contínua.

Exemplo 3.1.2 Consideremos $\sigma=\left\langle v_{0} v_{1} v_{2} v_{3}\right\rangle, \tau=\left\langle w_{0} w_{1}\right\rangle$ e $f: \sigma \rightarrow \tau$ a. aplicação simplicial definida por: $f\left(v_{0}\right)=w_{0}, f\left(v_{1}\right)=f\left(v_{2}\right)=f\left(v_{3}\right)=w_{1}$. 


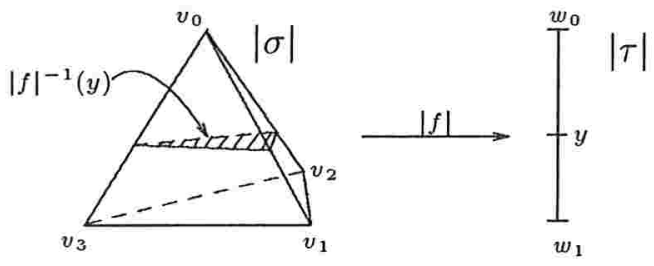

Seja $y \in \stackrel{\circ}{\tau}$; pelos Corolários 2.2.2, 2.2.4 e pela Proposição 2.2.1, a fibra $|f|^{-1}(y)$ é conexa, $\operatorname{dim}|f|^{-1}(y)=3-1=2$ e $|f|^{-1}(y)$ é homeomorfo a $\sigma_{0} \times \sigma_{1}$, onde $\sigma_{0}=\left\langle v_{0}\right\rangle$ e $\sigma_{1}=\left\langle v_{1} v_{2} v_{3}\right\rangle$. Pela. Proposição 3.1.1, temos que $W_{|f|}$ é homeomorfo a $\tau$.

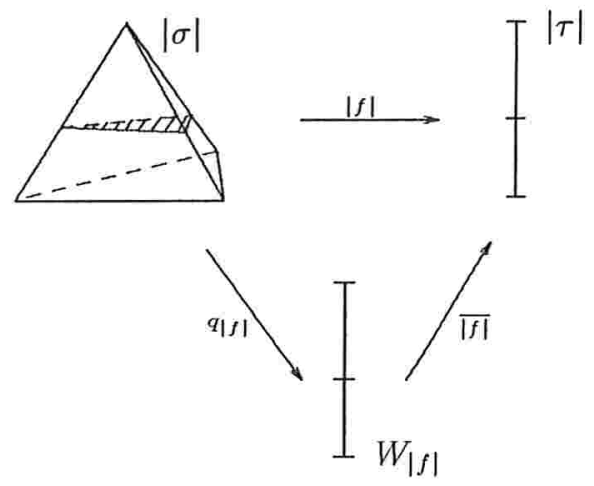

Exemplo 3.1.3 Consideremos $\sigma=\left\langle v_{0} v_{1} v_{2} v_{3}\right\rangle, \tau=\left\langle w_{0} w_{1} w_{2}\right\rangle$ e $f: \sigma \rightarrow \tau$ a aplicação simplicial definida por: $f\left(v_{0}\right)=w_{0}, f\left(v_{1}\right)=w_{1}, f\left(v_{2}\right)=f\left(v_{3}\right)=$ $w_{2}$.

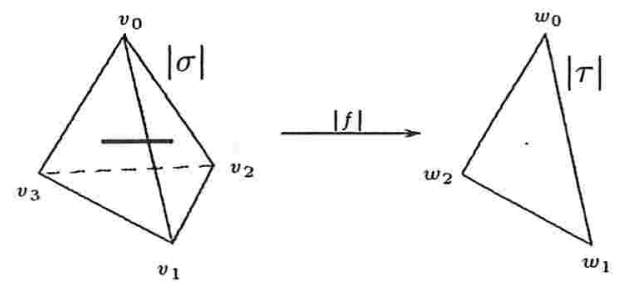


Seja $y \in \stackrel{\circ}{\tau}$, pelos Corolários 2.2.2, 2.2.4 e pela Proposição 2.2.1, a fibra $|f|^{-1}(y)$ é conexa, $\operatorname{dim}|f|^{-1}(y)=3-2=1$ e $|f|^{-1}(y)$ é homeomorfo a $\sigma_{0} \times \sigma_{1} \times \sigma_{2}$, onde $\sigma_{0}=\left\langle v_{0}\right\rangle, \sigma_{1}=\left\langle v_{1}\right\rangle$ e $\sigma_{2}=\left\langle v_{2} v_{3}\right\rangle$. Pela Proposição 3.1.1, temos que $W_{|f|}$ é homeomorfo a $\tau$.

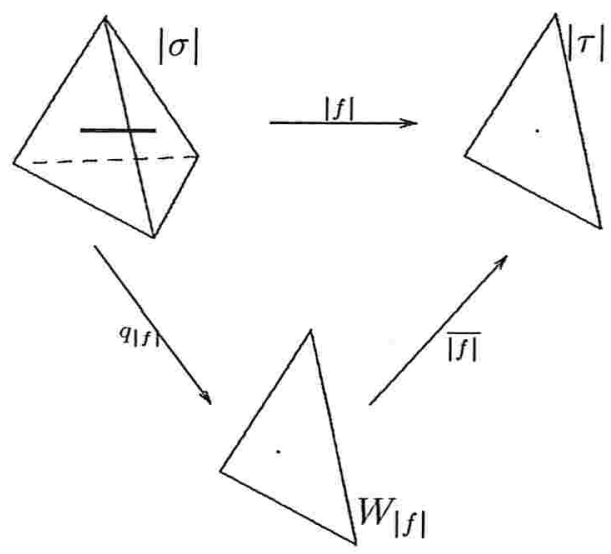

Exemplo 3.1.4 Seja $K$ um complexo simplicial gerado pelos 2-simplexos: $\sigma_{0}=\left\langle v_{1} v_{2} v_{3}\right\rangle, \sigma_{1}=\left\langle v_{0} v_{2} v_{3}\right\rangle, \sigma_{2}=\left\langle v_{0} v_{1} v_{3}\right\rangle, \sigma_{3}=\left\langle v_{0} v_{1} v_{2}\right\rangle$. Sejam, ainda, o 1-simplexo $\tau=\left\langle w_{0} w_{1}\right\rangle$ e $f: K \rightarrow \tau$ a aplicação simplicial definida por: $f\left(v_{0}\right)=w_{0}, f\left(v_{1}\right)=f\left(v_{2}\right)=f\left(v_{3}\right)=w_{1}$.

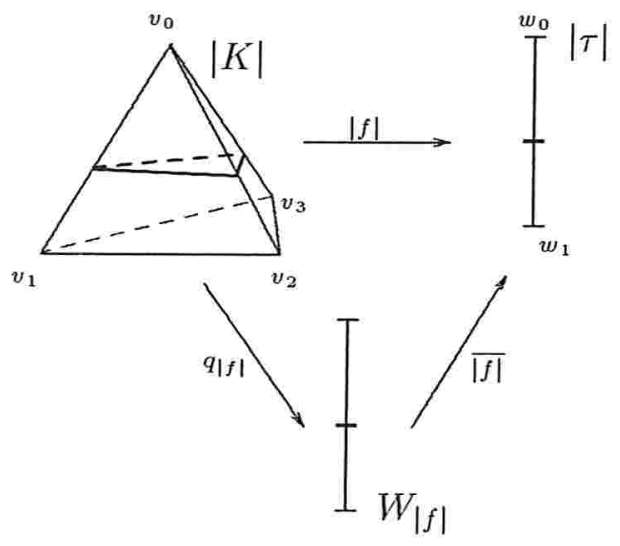


Se $y \in \stackrel{\circ}{\tau}$, então a fibra $|f|^{-1}(y)$ é conexa e $\operatorname{dim}|f|^{-1}(y)=2-1=1$. Pela Proposição 3.1.1, temos que $W_{|f|}$ é homeomorfo a $\tau$.

O exemplo abaixo mostra claramente que, para que a fatorização de Stein de uma aplicação simplicial seja um complexo simplicial, é necessário que consideremos pelo menos a primeira subdivisão baricêntrica (Observação 1.1.17 e Definição 1.2.7) da imagem da aplicação simplicial.

Exemplo 3.1.5 Seja $K$ um complexo simplicial 2-dimensional gerado pelos simplexos $\sigma_{0}=\left\langle v_{1} v_{2} v_{3}\right\rangle, \sigma_{1}=\left\langle v_{0} v_{2} v_{3}\right\rangle, \sigma_{2}=\left\langle v_{0} v_{1} v_{3}\right\rangle, \sigma_{3}=\left\langle v_{0} v_{1} v_{2}\right\rangle$. Consideremos, ainda, $\tau=\left\langle w_{0} w_{1} w_{2}\right\rangle$ um 2-simplexo e $f: K \rightarrow \tau$ a aplicação simplicial definida por $f\left(v_{0}\right)=w_{0}, f\left(v_{1}\right)=w_{1}, f\left(v_{2}\right)=f\left(v_{3}\right)=w_{2}$.

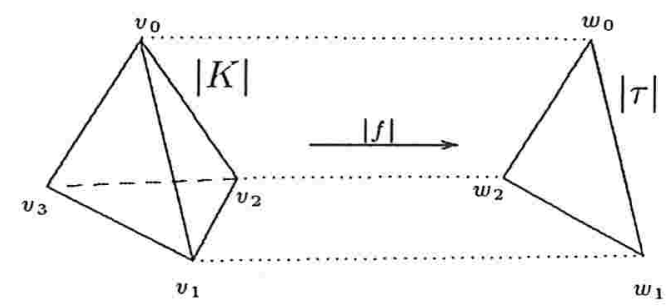

Para qualquer $y \in \stackrel{\circ}{\tau}, y=\sum_{k=0}^{2} \beta_{k} w_{k}, \beta_{k}>0, \sum_{k=0}^{2} \beta_{k}=1$, temos que

$$
|f|^{-1}(y)=\left\{\alpha_{0} v_{0}+\alpha_{1} v_{1}+\alpha_{2} v_{2}\right\} \cup\left\{\alpha_{0} v_{0}+\alpha_{1} v_{1}+\alpha_{2} v_{3}\right\},
$$

onde $\alpha_{i}=\beta_{i}, i=0,1,2$. Ou seja, as fibras têm duas componentes conexas para qualquer $y \in \stackrel{\circ}{\mathcal{T}}$.

Considere o diagrama comutativo:

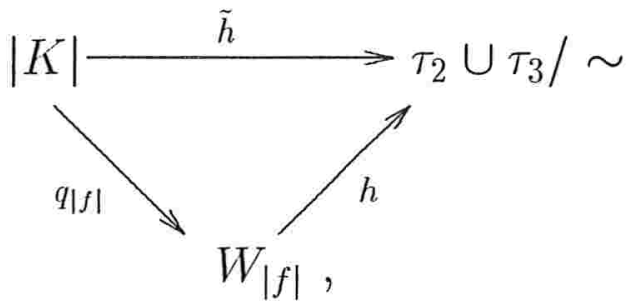


onde $\tau_{2}$ e $\tau_{3}$ são cópias de $\tau,\left.\tilde{h}\right|_{\sigma_{i}}=|f|_{\sigma_{i}}: \sigma_{i} \rightarrow \tau_{i}=\tau$ para $i=2,3$, $\left.\tilde{h}\right|_{|f|^{-1}(\partial \tau)}=|f|_{|f|^{-1}(\partial \tau)}, \sim$ é a relação de equivalência que relaciona apenas os pontos de $\partial \tau$, e $h$ é a aplicação definida da maneira mais natural. Então $\tilde{h}$ está bem definida e é contínua pelo Lema 1.1.14. Conseqüentemente, $h$ também é contínua pela definição da topologia em $W_{|f|}$ e é uma bijeção. Como $|K|$ é compacto, $W_{|f|}=q_{|f|}(|K|)$ também é compacto; além disso, $\tau_{2} \cup \tau_{3} / \sim$ é Hausdorff. Então, $h$ é um homeomorfismo.

$\mathrm{O}$ espaço $\tau_{2} \cup \tau_{3} / \sim$ pode ser considerado como o espaço subjacente de algum complexo simplicial, mas este complexo simplicial não pode ser gerado por $\tau_{2}$ e $\tau_{3}$, pois eles têm exatamente os mesmos vértices, mas são simplexos distintos. Portanto, uma subdivisão baricêntrica na imagem de $\tilde{h}$ vai ser necessária para que $\tau_{2} \cup \tau_{3} / \sim$ seja homeomorfo a um poliedro.

Uma possível representação $V$ para a vizualização de $W_{|f|}$ é a seguinte. Consideremos a imagem de $\tau$ através do mergulho $\mathbb{R}^{2} \stackrel{\varphi}{\longrightarrow} \mathbb{R}^{3},(a, b) \mapsto$ $(a, b, 0)$. Considere pontos da forma $\left(0,0, c_{i}\right), i=2,3$, com $c_{2}>0$ e $c_{3}<0$.

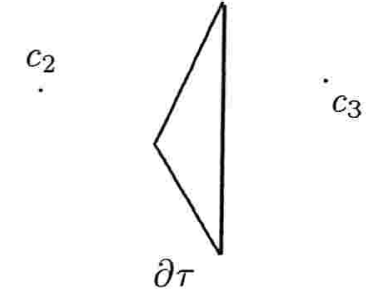

Seja $\bar{\tau}_{i}$ o cone $c_{i} \varphi(\partial \tau), i=2,3$, e $V=\bar{\tau}_{2} \cup \bar{\tau}_{3}$. Então $V$ é homeomorfo a $W_{|f|}$, pois $\tau_{2} \cup \tau_{3} / \sim$ é homeomorfo a $V$.

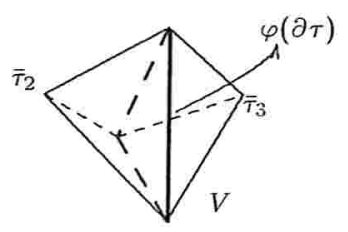




\subsection{Complexo simplicial correspondente à fa- torização de Stein}

Dada $\tilde{f}: \tilde{K} \rightarrow \tilde{L}$ uma aplicação simplicial entre dois complexos simpliciais localmente finitos, se $L$ é a subdivisão baricêntrica do complexo simplicial $\tilde{L}$, pela Proposição 1.1.19, existe uma subdivisão $K$ de $\tilde{K}$ e uma aplicação simplicial $f: K \rightarrow L$ tal que $|f|=|\tilde{f}|$, onde $|f|:|K| \rightarrow|L|$ e $|\tilde{f}|:|\tilde{K}| \rightarrow|\tilde{L}|$.

Vamos definir um complexo simplicial abstrato $\mathcal{V}$ e mostrar que o espaço subjacente de sua realização geométrica é homeomorfo a $W_{|\tilde{f}|}=W_{|f|}$. No decorrer deste capítulo, assumiremos que os complexos simpliciais $\tilde{K}$ e $\tilde{L}$ são localmente finitos (e, conseqüentemente, $K$ e $L$ também o são pelo Lema 1.1.18) e que a aplicação $|f|=|\tilde{f}|:|K| \rightarrow|L|$ é própria. Iniciamos com a seguinte:

Definição 3.2.1 Sejam $v, w \in K^{(0)}$. Dizemos que $v$ é equivalente a $w$, e denotamos por $v \sim w$, se $f(v)=f(w)$ e existe uma seqüência finita de vértices $v_{0}, v_{1}, \ldots, v_{s}$ de $f^{-1}(f(v))=f^{-1}(f(w)) \subset K$ tal que $v=v_{0}, w=v_{s}$ $\mathrm{e}\left\langle v_{i} v_{i+1}\right\rangle \in f^{-1}(f(v)), 0 \leq i \leq s-1$.

Observação 3.2.2 A relação definida acima é uma relação de equivalência. Se $v \sim w$, então eles pertencem à mesma componente conexa da fibra $|f|^{-1}(|f|(v))=|f|^{-1}(|f|(w))$. Note que a Definição 3.2.1 depende somente das aplicações simpliciais e não depende da aplicação contínua entre os espaços subjacentes.

A partir de $K$ definimos um complexo simplicial abstrato $\mathcal{V}$ da seguinte forma: 
Definição 3.2.3 Sejam $\mathcal{V}^{(0)}=K^{(0)} / \sim$ o conjunto dos vértices e $\left[v_{0}\right], \ldots,\left[v_{k}\right]$ classes distintas de $\mathcal{V}^{(0)}$; dizemos que $\left\{\left[v_{0}\right], \ldots,\left[v_{k}\right]\right\}$ é um simplexo de dimensão $k$ ou, simplesmente, um $k$-simplexo de $\mathcal{V}$ se para cada $i=0, \ldots, k$, existe $v_{i}^{\prime} \in\left[v_{i}\right]$ tal que $\left\langle v_{0}^{\prime} \cdots v_{k}^{\prime}\right\rangle$ é um $k$-simplexo de $K$.

O conjunto $\mathcal{V}$ é um complexo simplicial abstrato. De fato, seja $\sigma=$ $\left\{\left[v_{0}\right], \ldots,\left[v_{k}\right]\right\} \in \mathcal{V}$, então existem $v_{i}^{\prime} \in\left[v_{i}\right]$, para $i=0, \ldots, k$, tais que $\left\langle v_{0}^{\prime} \cdots v_{k}^{\prime}\right\rangle \in K$. Se $\left\{\left[v_{i_{0}}\right], \ldots,\left[v_{i_{p}}\right]\right\}$ é um subconjunto de $\sigma$, então $\left\langle v_{i_{0}}^{\prime} \ldots v_{i_{p}}^{\prime}\right\rangle$ é uma face de $\left\langle v_{0}^{\prime} \ldots v_{k}^{\prime}\right\rangle$ e $\left\langle v_{i_{0}}^{\prime} \cdots v_{i_{p}}^{\prime}\right\rangle \in K$, pois $K$ é um complexo simplicial. Portanto, $\left\{\left[v_{i_{0}}\right], \ldots,\left[v_{i_{p}}\right]\right\} \in \mathcal{V}$.

Seja $\mathcal{K}$ o esquema de vértices de $K$. Definimos a aplicação $\varphi: \mathcal{K} \rightarrow \mathcal{V}$ da seguinte forma: $\varphi(v)=[v]$ e $\varphi\left(\left\{v_{0}, \ldots, v_{k}\right\}\right)=\left\{\left[v_{0}\right], \ldots,\left[v_{k}\right]\right\}$.

Observação 3.2.4 (1) $\varphi\left(\left\{v_{0}, \ldots, v_{k}\right\}\right)$ pode não ser um $k$-simplexo, ou seja, $\left[v_{0}\right], \ldots,\left[v_{k}\right]$ pode ter repetições e $\left\{\left[v_{0}\right], \ldots,\left[v_{k}\right]\right\}$ pode ter dimensão menor do que $k$;

(2) $\varphi$ é simplicial.

Agora, considerando $\mathcal{L}$ o esquema de vértices de $L$, definimos a aplicação $\psi: \mathcal{V} \rightarrow \mathcal{L}$ da seguinte forma: $\psi([v])=f(v)$ e $\psi\left(\left\{\left[v_{0}\right], \ldots,\left[v_{k}\right]\right\}\right)=$ $\left\{f\left(v_{0}\right), \ldots, f\left(v_{k}\right)\right\}$. A aplicação $\psi$ está bem definida, pois $f(v)$ não depende da escolha do representante $v$ da classe $[v]$ e $\left\{f\left(v_{0}\right), \ldots, f\left(v_{k}\right)\right\}$ é um simplexo de $L$. Veremos maiores detalhes sobre $\psi$ no Lema 3.2 .6 abaixo.

Observação 3.2.5 Por um abuso de notação, usaremos a mesma letra $f$ para denotar as aplicações:

1) $f: K \rightarrow L$ entre complexos simplicias geométricos;

2) $f: K^{(0)} \rightarrow L^{(0)}$ a aplicação restrita ao conjunto dos vértices de $K$;

3) $f: \mathcal{K} \rightarrow \mathcal{L}$ entre os esquemas de vértices de $K$ e $L$. 
Porém, continuaremos denotando por $|f|:|K| \rightarrow|L|$ a aplicação entre os espaços subjacentes de $K$ e $L$.

Lema 3.2.6 (1) $\psi$ está bem definida e é simplicial.

(2) $\psi$ é não-degenerada, isto é, $\operatorname{dim}(\psi(\sigma))=\operatorname{dim} \sigma$, para qualquer $\sigma \in \mathcal{V}$.

(3) O seguinte diagrama é comutativo:

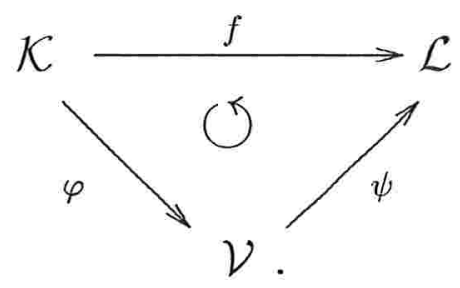

\section{Demonstração:}

(1) De fato, sejam $v$ e $v^{\prime}$ dois representantes quaisquer da classe $[v]$, temos que $v \sim v^{\prime}$, ou seja, $f(v)=f\left(v^{\prime}\right)$ e isto implica que $\psi([v])=\psi\left(\left[v^{\prime}\right]\right)$. Analogamente, sejam $v_{i}, v_{i}^{\prime}$ dois representantes da classe $\left[v_{i}\right]$, para $i=$ $0, \ldots, k$, então $f\left(v_{i}\right)=f\left(v_{i}^{\prime}\right)$ e concluímos que $\psi\left(\left\{\left[v_{0}\right], \ldots,\left[v_{k}\right]\right\}\right)=$ $\psi\left(\left\{\left[v_{0}^{\prime}\right], \ldots,\left[v_{k}^{\prime}\right]\right\}\right)$. Consideremos agora $\left\{\left[v_{0}\right], \ldots,\left[v_{k}\right]\right\}$ um simplexo de $\mathcal{V}$; então existem $v_{i}^{\prime} \in\left[v_{i}\right], i=0, \ldots, k$, tais que $\left\{v_{0}^{\prime}, \ldots, v_{k}^{\prime}\right\}$ é um simplexo de $\mathcal{K}$. Como $f$ é simplicial, temos que $\left\{f\left(v_{0}^{\prime}\right), \ldots, f\left(v_{k}^{\prime}\right)\right\} \in$ $\mathcal{L}$. Mas $f\left(v_{i}^{\prime}\right)=f\left(v_{i}\right)$, para todo $i=0, \ldots, k$, e isto implica que $\left\{f\left(v_{0}\right), \ldots, f\left(v_{k}\right)\right\}=\psi\left(\left\{\left[v_{0}\right], \ldots,\left[v_{k}\right]\right\}\right) \in \mathcal{L}$ e assim $\psi$ é simplicial.

(2) Basta mostrar que a imagem de qualquer 1-simplexo de $\mathcal{V}$, através de $\psi$, é um 1-simplexo de $\mathcal{L}$.

Seja $\sigma=\left\{\left[v_{0}\right],\left[v_{1}\right]\right\}$ um 1-simplexo de $\mathcal{V}$. Pela Definição 3.2.3, existem $v_{i}^{\prime} \in\left[v_{i}\right], i=0,1$, tais que $\left\langle v_{0}^{\prime}, v_{1}^{\prime}\right\rangle \in K$. Mais ainda, pela Definição 1.2.3, $\left\{v_{0}^{\prime}, v_{1}^{\prime}\right\} \in \mathcal{K}$. Como $\psi$ não depende dos representantes, 
podemos considerar $\psi\left(\left\{\left[v_{0}\right],\left[v_{1}\right]\right\}\right)=\left\{f\left(v_{0}^{\prime}\right), f\left(v_{1}^{\prime}\right)\right\}$. Suponhamos, por absurdo, que $f\left(v_{0}^{\prime}\right)=f\left(v_{1}^{\prime}\right)$, ou seja, $\psi(\sigma)$ é um 0 -simplexo de $\mathcal{L}$. Observemos que, como $\left\langle v_{0}^{\prime}, v_{1}^{\prime}\right\rangle \in K$ e $f\left(v_{0}^{\prime}\right)=f\left(v_{1}^{\prime}\right)$, pela definição de aplicação simplicial, segue que $\left\langle v_{0}^{\prime}, v_{1}^{\prime}\right\rangle \in f^{-1}\left(f\left(v_{0}^{\prime}\right)\right)=f^{-1}\left(f\left(v_{1}^{\prime}\right)\right)$. Com isto, temos satisfeita a Definição 3.2.1, ou seja, $v_{0}^{\prime} \sim v_{1}^{\prime}$, o que é uma contradição, pois $\left[v_{0}^{\prime}\right] \neq\left[v_{1}^{\prime}\right]$. Portanto, $\psi$ é não-degenerada.

(3) Para qualquer simplexo $\sigma=\left\{v_{0}, \ldots, v_{k}\right\}$ de $\mathcal{K}$, temos

$$
\begin{aligned}
\psi\left(\varphi\left(\left\{v_{0}, \ldots, v_{k}\right\}\right)\right) & =\psi\left(\left\{\left[v_{0}\right], \ldots,\left[v_{k}\right]\right\}\right) \\
& =\left\{f\left(v_{0}\right), \ldots, f\left(v_{k}\right)\right\} \\
& =f\left(\left\{v_{0}, \ldots, v_{k}\right\}\right) .
\end{aligned}
$$

Portanto, $\psi(\varphi(\sigma))=f(\sigma), \forall \sigma \in \mathcal{K}$, ou seja, o diagrama (3.4) é comutativo.

Seja $L$ a subdivisão baricêntrica do complexo simplicial abstrato $\tilde{L}$. A cada vértice $v$ de $L^{(0)}$ corresponde um único simplexo $\sigma^{k}$ de $\tilde{L}$. Denotaremos por $\sigma_{j}^{i_{j}}$, somente no lema abaixo, os vértices de $L^{(0)}$, onde $i_{j}$ é a dimensão de $\sigma_{j}^{i_{j}}$ como simplexo de $\tilde{L}$.

Lema 3.2.7 Consideremos $\tau=\left\{\sigma_{0}^{i_{0}}, \sigma_{1}^{i_{1}}, \ldots, \sigma_{k}^{i_{k}}\right\}$ um $k$-simplexo de $L$, onde $i_{0} \leq i_{1} \leq \cdots \leq i_{k}$, então:

(1) $i_{j}<i_{l}$, para todo $j<l$;

(2) existe um único simplexo $\tilde{\tau} \in \tilde{L}$ tal que $\stackrel{\circ}{\tau} \subset \stackrel{\circ}{\tau}$;

(3) $\operatorname{dim} \tau \leq \operatorname{dim} \tilde{\tau}$.

\section{Demonstração:}

(1) Pela Definição 1.2.7, $\tau$ é um simplexo de $L$ se, e somente se, $\sigma_{0}^{i_{0}} \ll$ $\sigma_{1}^{i_{1}} \ll \cdots \ll \sigma_{k}^{i_{k}}$. Portanto, $i_{0}<i_{1}<\cdots<i_{k}$. 
(2) Seja $\tau=\left\{\sigma_{0}^{i_{0}}, \sigma_{1}^{i_{1}}, \ldots, \sigma_{k}^{i_{k}}\right\}$ um $k$-simplexo de L. Pela Definição 1.2.7, temos que $\sigma_{0}^{i_{0}} \ll \sigma_{1}^{i_{1}} \ll \cdots \ll \sigma_{k}^{i_{k}}$. Como todos os vértices de $\tau$ satisfazem a relação acima, temos que $\sigma_{0}^{i_{0}} \subset \sigma_{1}^{i_{1}} \subset \cdots \subset \sigma_{k}^{i_{k}}$. Seja $\tilde{\tau}=\sigma_{k}^{i_{k}}$, assim temos, novamente pela Definição 1.2.7 e pela Observação 1.1.17, que $\stackrel{\circ}{\tau} \subset \tilde{\tau}$. Se existe $\tilde{\theta} \in \tilde{L}$ tal que $\stackrel{\circ}{\tau} \subset \tilde{\theta}$, então $\stackrel{\circ}{\tilde{\tau}} \cap \stackrel{\circ}{\tilde{\theta}} \supset \stackrel{\circ}{\tau} \neq \emptyset$, e isto implica que $\tilde{\tau}=\tilde{\theta}$, pois $\tilde{L}$ é um complexo simplicial.

(3) Pelo item anterior, $\stackrel{\circ}{\tau} \subset \stackrel{\circ}{\tilde{\tau}}$, e portanto, $\operatorname{dim} \tau \leq \operatorname{dim} \tilde{\tau}$.

Lema 3.2.8 Seja $V$ a realização geométrica de $\mathcal{V}$. Consideremos as aplicações $\varphi$ e $\psi$ definidas acima e as correspondentes aplicações dos seus espaços subjacentes: $|\varphi|:|K| \rightarrow|V|$ e $|\psi|:|V| \rightarrow|L|$. Então, para todo $x \in|V|,|\varphi|^{-1}(x)$ está contido em uma componente conexa de $|f|^{-1}(|\psi|(x))$.

\section{Demonstração:}

Primeiramente consideremos o caso em que $x$ é um vértice de $V$, ou seja, $x=[v]$, para algum $v \in K^{(0)}$.

Para todo $u \in\left(\varphi^{-1}([v])\right)^{(0)}$, temos $[u]=\varphi(u)=[v]$; logo $u$ e $v$ pertencem à mesma componente conexa de $|f|^{-1}(|f|(u))=|f|^{-1}(|f|(v))=$ $|f|^{-1}(|\psi| \circ|\varphi|(v))=|f|^{-1}(|\psi|([v]))=|f|^{-1}(|\psi|(x))$.

Consideremos agora $x$ um ponto qualquer de $|V|$; então existe um único simplexo abstrato $\sigma=\left\{\left[v_{0}\right], \ldots,\left[v_{k}\right]\right\}$ pertencente a $\mathcal{V}$ de dimensão $k$ tal que $x \in \stackrel{\circ}{\sigma}$.

Seja $\tau=\psi(\sigma) \in \mathcal{L}$, então $\tau$ é da forma:

$$
\left\{\psi\left(\left[v_{0}\right]\right), \ldots, \psi\left(\left[v_{k}\right]\right)\right\}=\left\{f\left(v_{0}\right), \ldots, f\left(v_{k}\right)\right\}
$$

Pelo item (2) do Lema 3.2.6, sabemos que $\psi$ é não-degenerada, ou seja, $\operatorname{dim} \tau=k ; \operatorname{logo}|\psi|(x) \in \stackrel{\circ}{\tau}$. Como $L$ é a subdivisão baricêntrica de $\tilde{L}$, pelo

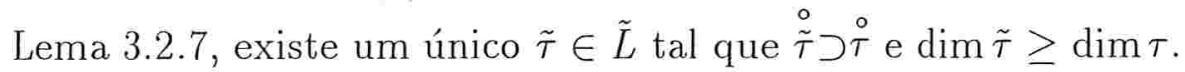




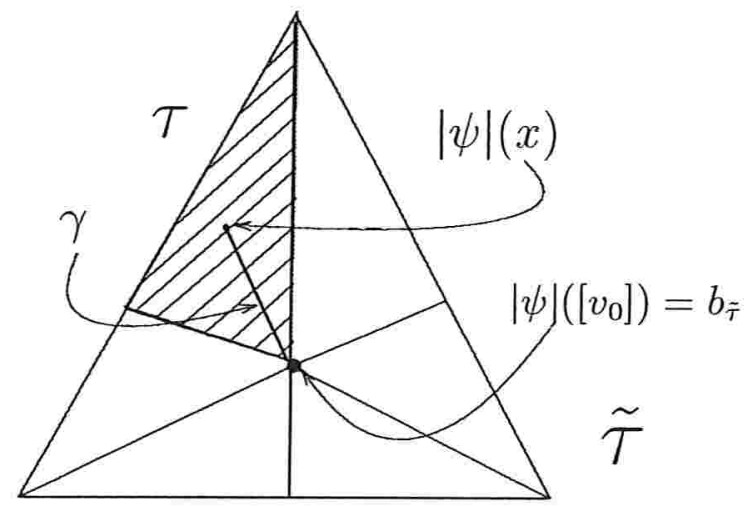

Podemos supor, sem perda de generalidade, que $|\psi|\left(\left[v_{0}\right]\right)$ é o baricentro de $\tilde{\tau}$, isto é $|\psi|\left(\left[v_{0}\right]\right)=b_{\tilde{\tau}}$. ;

Consideremos agora $\gamma$ o segmento em $\tau$ ligando $|\psi|(x)$ e $|\psi|\left(\left[v_{0}\right]\right)$; notemos que $\gamma$ está inteiramente contido no interior de $\tilde{\tau}$. Como $|f|=|\tilde{f}|$, aplicando a Proposição 2.3.9, temos um homeomorfismo $H_{\gamma}:|f|^{-1}(\gamma) \rightarrow|f|^{-1}\left(b_{\bar{\tau}}\right) \times \gamma$ que faz o seguinte diagrama comutar:

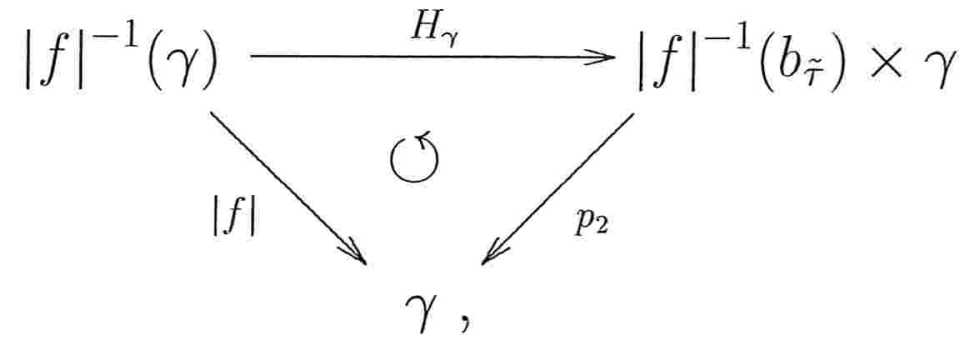

onde $p_{2}(a, c)=c$, para $\forall(a, c) \in|f|^{-1}\left(b_{\tilde{\tau}}\right) \times \gamma$.

Para concluir a demonstração, basta mostrar que dois pontos quaisquer $y$ e $z$ de $|\varphi|^{-1}(x)$ estão na mesma componente conexa de $|f|^{-1}(|\psi|(x))$.

Existem dois simplexos $\sigma_{y}=\left\langle u_{0} \cdots u_{l}\right\rangle$ e $\sigma_{z}=\left\langle w_{0} \cdots w_{m}\right\rangle$ de $K$, tais que $y \in \stackrel{\circ}{\sigma}_{y}, z \in \stackrel{\circ}{\sigma}_{z}$ e $\varphi\left(\sigma_{y}\right)=\sigma=\varphi\left(\sigma_{z}\right)$ (veja Lema 2.3.7). 
Suponhamos agora,

$$
\begin{gathered}
\varphi\left(u_{0}\right)=\cdots=\varphi\left(u_{i_{0}}\right)=\left[v_{0}\right]=\varphi\left(w_{0}\right)=\cdots=\varphi\left(w_{j_{0}}\right) \\
\varphi\left(u_{i_{0}+1}\right)=\cdots=\varphi\left(u_{i_{1}}\right)=\left[v_{1}\right]=\varphi\left(w_{j_{0}+1}\right)=\cdots=\varphi\left(w_{j_{1}}\right) \\
\vdots \\
\vdots \\
\varphi\left(u_{i_{k-1}+1}\right)=\cdots=\varphi\left(u_{l}\right)=\left[v_{k}\right]=\varphi\left(w_{j_{k-1}+1}\right)=\cdots=\varphi\left(w_{m}\right) .
\end{gathered}
$$

Portanto, temos $u_{0} \sim \cdots \sim u_{i_{0}} \sim w_{0} \sim \cdots \sim w_{j_{0}}$, implicando que eles estão na mesma componente conexa de $|f|^{-1}\left(|f|\left(v_{0}\right)\right)=|f|^{-1}\left(|\psi|\left(\left[v_{0}\right]\right)\right)=$ $|f|^{-1}\left(b_{\bar{\tau}}\right)$, ou seja, eles estão na mesma componente conexa de $|f|^{-1}(\gamma)$.

Consideremos agora $\gamma_{y}$ o segmento em $\sigma_{y}$ ligando $u_{0}$ a $y$, e $\gamma_{z}$ o segmento em $\sigma_{z}$ ligando $w_{0}$ a $z$. Temos que $|f|\left(\gamma_{y}\right)=|f|\left(\gamma_{z}\right)=\gamma$. Então, $u_{0}$ e $y$ estão na mesma componente conexa de $|f|^{-1}(\gamma)$, assim como $w_{0}$ e $z$ também estão na mesma componente conexa de $|f|^{-1}(\gamma)$.

Logo, $y$ e $z$ estão na mesma componente conexa $C$ de $|f|^{-1}(\gamma)$. Pelo diagrama comutativo (3.5) temos que $C$ é homeomorfo a $\gamma \times c$, onde $c$ é a componente conexa de $|f|^{-1}\left(b_{\tilde{\tau}}\right)$ que contém $u_{0}$ e $w_{0}$. Em particular, $|f|^{-1}(|\psi|(x)) \cap C$ é homeomorfo a $c$, que é conexo. Mais ainda, $y, z \in|f|^{-1}(|\psi|(x)) \cap C$; portanto $y$ e $z$ pertencem à mesma componente conexa de $|f|^{-1}(|\psi|(x))$.

Vamos mostrar agora que o espaço subjacente, $|V|$, associado ao complexo simplicial abstrato $\mathcal{V}$ é homeomorfo a $W_{|f|}$. Para tanto, iniciamos definindo a aplicação $k:|V| \rightarrow W_{|f|}$ por $k(x)=q_{|f|}\left(|\varphi|^{-1}(x)\right)$, onde $q_{|f|}:|K| \rightarrow W_{|f|}$ é a aplicação quociente associada à $|f|:|K| \rightarrow|L|$.

A aplicação $k$ está bem definida. De fato, pelo Lema 3.2.8, segue que $|\varphi|^{-1}(x)$ está contida em uma componente conexa de $|f|^{-1}(|\psi|(x))$. Portanto, pela definição de $q_{|f|}$, temos que $|\varphi|^{-1}(x)$ se projeta em um único ponto de $W_{|f|}$ através de $q_{|f|}$.

Temos ainda o seguinte diagrama comutativo: 


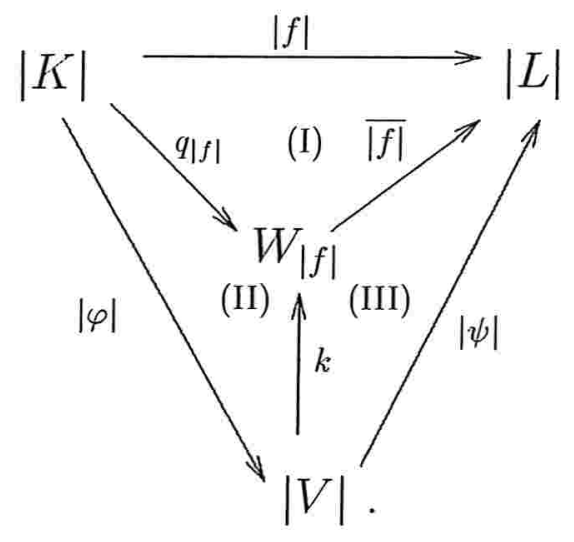

Já vimos anteriormente que (I) é comutativo. Vamos verificar a comutatividade de (II). Seja $x$ um ponto qualquer de $|K|$ e consideremos a composta $k(|\varphi|(x))=q_{|f|}\left(|\varphi|^{-1}(|\varphi|(x))\right)$. Utilizando o Lema 3.2.8, temos que $|\varphi|^{-1}(|\varphi|(x))$ está contido em uma componente conexa de $|f|^{-1}(|\psi|(|\varphi|(x)))=$ $|f|^{-1}(|f|(x))$, ou seja, $q_{|f|}\left(|\varphi|^{-1}(|\varphi|(x))\right)=q_{|f|}(z)$, onde $z$ é um ponto qualquer da componente conexa de $|f|^{-1}(|\psi|(|\varphi|(x)))$ que contém $|\varphi|^{-1}(|\varphi|(x))$. Em particular, $x$ pertence a esta componente conexa. Portanto, temos que $q_{|f|}\left(|\varphi|^{-1}(|\varphi|(x))\right)=q_{|f|}(x)$, ou seja, $k(|\varphi|(x))=q_{|f|}(x), \forall x \in|K|$. Portanto, (II) é comutativo.

Finalmente, para mostrar que (III) é comutativo, consideremos a composta $\overline{|f|}(k(y))$, onde $y$ é um ponto qualquer de $|V|$. Pela definição de $k$ e a comutatividade de (I) temos que:

$$
\overline{|f|}(k(y))=\overline{|f|}\left(q_{|f|}\left(|\varphi|^{-1}(y)\right)\right)=|f|\left(|\varphi|^{-1}(y)\right)=|\psi|(y)
$$

onde a última igualdade é decorrente do Lema 3.2.8. Portanto, (III) é comutativo.

Para definirmos a inversa de $k$ necessitamos do seguinte: 
Lema 3.2.9 Para todo $x \in W_{|f|}$, o conjunto $|\varphi|\left(q_{|f|}^{-1}(x)\right)$ consiste de apenas um ponto.

\section{Demonstração:}

Fixado um $x \in W_{|f|}$, sejam $y$ e $z$ pontos quaisquer em $q_{|f|}^{-1}(x)$. Pela comutatividade (I) do diagrama (3.6), temos que:

$$
|f|(y)=\overline{|f|} \circ q_{|f|}(y)=\overline{|f|}(x)=\overline{|f|} \circ q_{|f|}(z)=|f|(z)
$$

Portanto, existe um único $\tau=\left\langle v_{0} \cdots v_{k}\right\rangle \in L$ tal que $|f|(y)=|f|(z) \in \stackrel{\circ}{\tau}$; também existem $\sigma_{y}=\left\langle u_{0} \cdots u_{l}\right\rangle \in K, \sigma_{z}=\left\langle w_{0} \cdots w_{m}\right\rangle \in K$, com $l, m \geq k$, tais que $y \in \circ_{y}^{\circ}, z \in \circ_{z}^{\circ}$ e $f\left(\sigma_{y}\right)=f\left(\sigma_{z}\right)=\tau$ (veja Lema 2.3.7).

Suponhamos

$$
\begin{gathered}
f\left(u_{0}\right)=\cdots=f\left(u_{i_{0}}\right)=v_{0}=f\left(w_{0}\right)=\cdots=f\left(w_{j_{0}}\right), \\
f\left(u_{i_{0}+1}\right)=\cdots=f\left(u_{i_{1}}\right)=v_{1}=f\left(w_{j_{0}+1}\right)=\cdots= \\
\vdots \\
\vdots \\
f\left(u_{i_{k-1}+1}\right)=\cdots=f\left(u_{l}\right)=v_{k}=f\left(w_{j_{k-1}+1}\right)=\cdots=
\end{gathered}
$$

Como $u_{0}, \ldots, u_{l}$ são vértices do simplexo $\sigma_{y} \in K$, temos:

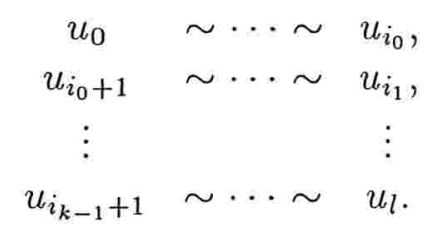

Analogamente, temos:

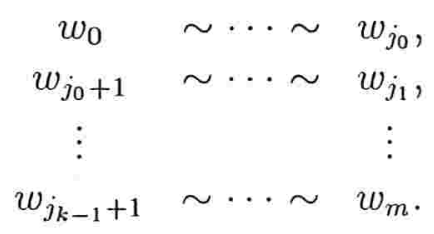

É suficiente mostrar agora que: 


$$
u_{0} \sim w_{0}, u_{i_{0}+1} \sim w_{j_{0}+1}, \ldots, u_{i_{k-1}+1} \sim w_{j_{k-1}+1},
$$

pois escrevendo $y$ e $z$ em coordenadas baricêntricas obtemos:

$$
y=\sum_{r=0}^{k} \sum_{i=i_{r-1}+1}^{i_{r}} \alpha_{i} u_{i} \quad \text { e } \quad z=\sum_{r=0}^{k} \sum_{j=j_{r-1}+1}^{j_{r}} \beta_{j} w_{j}
$$

onde $i_{-1}=-1=j_{-1}, \alpha_{i}>0, \beta_{j}>0, \sum_{i=0}^{l} \alpha_{i}=1$ e $\sum_{j=0}^{m} \beta_{j}=1$. De onde segue que:

$$
\begin{aligned}
|\varphi|(y) & =\sum_{r=0}^{k} \sum_{i=i_{r-1}+1}^{i_{r}} \alpha_{i}|\varphi|\left(u_{i}\right) \\
& =\sum_{r=0}^{k} \sum_{i_{i=i_{r-1}+1}}^{i_{r}} \alpha_{i}\left[u_{i_{r-1}+1}\right] \\
& =\sum_{r=0}^{k}\left(\sum_{i=i_{r-1}+1}^{i_{r}} \alpha_{i}\right)\left[u_{i_{r-1}+1}\right] .
\end{aligned}
$$

Também, temos

$$
\begin{aligned}
|\varphi|(z) & =\sum_{r=0}^{k} \sum_{j=j_{r-1}+1}^{j_{r}} \beta_{j}|\varphi|\left(w_{j}\right) \\
& =\sum_{r=0}^{k} \sum_{j=j_{r-1}+1}^{j_{r}} \beta_{j}\left[w_{j_{r-1}+1}\right] \\
& =\sum_{r=0}^{k}\left(\sum_{j=j_{r-1}+1}^{j_{r}} \beta_{j}\right)\left[w_{j_{r-1}+1}\right] .
\end{aligned}
$$

Como

$$
\begin{aligned}
& |f|(y)=\sum_{r=0}^{k} \sum_{i=i_{r-1}+1}^{i_{r}} \alpha_{i} f\left(u_{i}\right)=\sum_{r=0}^{k}\left(\sum_{i=i_{r-1}+1}^{i_{r}} \alpha_{i}\right) v_{r} \\
& |f|(z)=\sum_{r=0}^{k} \sum_{j=j_{r-1}+1}^{j_{r}} \beta_{j} f\left(w_{j}\right)=\sum_{r=0}^{k}\left(\sum_{j=j_{r-1}+1}^{j_{r}} \beta_{j}\right) v_{r},
\end{aligned}
$$


e temos $|f|(y)=|f|(z)$, pela unicidade das coordenadas baricêntricas, segue que

$$
\sum_{i=i_{r-1}+1}^{i_{r}} \alpha_{i}=\sum_{j=j_{r-1}+1}^{j_{r}} \beta_{j}
$$

para todo $r$. Por isso, se $\left[u_{i_{r-1}+1}\right]=\left[w_{j_{r-1}+1}\right]$ para todo $r$, temos $|\varphi|(y)=$ $|\varphi|(z)$.

Portanto mostremos as equivalências (3.7). Pelo Lema 2.4.2, para $r=0, \ldots, k,|f|^{-1}\left(v_{r}\right)$ é um retrato forte de deformação de $|f|^{-1}\left(\left\{v_{r}\right\} \cup \stackrel{\circ}{\tau}\right)$. Seja $\gamma_{r}:|f|^{-1}\left(\left\{v_{r}\right\} \cup \stackrel{\circ}{\tau}\right) \rightarrow|f|^{-1}\left(v_{r}\right)$ uma retração, para $r=0, \ldots, k$.

Para um $r$ arbitrário existem:

(i) $\omega_{1}$, um segmento de reta em $\sigma_{y}$ unindo $u_{i_{r-1}+1}$ e $y$; $\omega_{1}$ está contido em $|f|^{-1}\left(\left\{v_{r}\right\} \cup \stackrel{\circ}{\tau}\right)$.

(ii) $\omega_{2}$, uma curva em $|f|^{-1}(\overline{|f|}(x))$ ligando $y$ e $z$, pois $y, z \in q_{|f|}^{-1}(x)$; $\omega_{2}$ está contido em $|f|^{-1}(\stackrel{\circ}{\tau})$.

(iii) $\omega_{3}$, um segmento de reta em $\sigma_{z}$ unindo $w_{j_{r-1}+1}$ e $z ; \omega_{3}$ está contido em $|f|^{-1}\left(\left\{v_{r}\right\} \cup \stackrel{\circ}{\tau}\right)$.

Seja $\omega$ a curva composta por $\omega_{1}, \omega_{2}$ e $\bar{\omega}_{3}$, onde $\bar{\omega}_{3}$ é a curva $\omega_{3}$ com a orientação oposta. Temos, então, uma curva $\omega$ contida em $|f|^{-1}\left(\left\{v_{r}\right\} \cup \stackrel{\circ}{\tau}\right)$ unindo $u_{i_{r-1}+1}$ e $w_{j_{r-1}+1}$. Disto segue que $\gamma_{r}$ o $\omega$ é uma curva em $|f|^{-1}\left(v_{r}\right)$ ligando $u_{i_{r-1}+1}$ e $w_{j_{r-1}+1}$. Agora, não é difícil modificar esta curva $\gamma_{r} \circ \omega$ fixando os dois extremos, para que só passe por simplexos de $f^{-1}\left(v_{r}\right)$ de dimensão 1.

Concluímos, assim, que $u_{i_{r-1}+1} \sim w_{j_{r-1}+1}$, para todo $r=0, \ldots, k$ pela Definição 3.2.1. 
Seja a aplicação $h: W_{|f|} \rightarrow|V|$ dada por $h(x)=|\varphi|\left(q_{|f|}^{-1}(x)\right)$. Do Lema 3.2.9, segue que $h$ está bem definida. Temos ainda que $h$ faz o seguinte diagrama comutar:

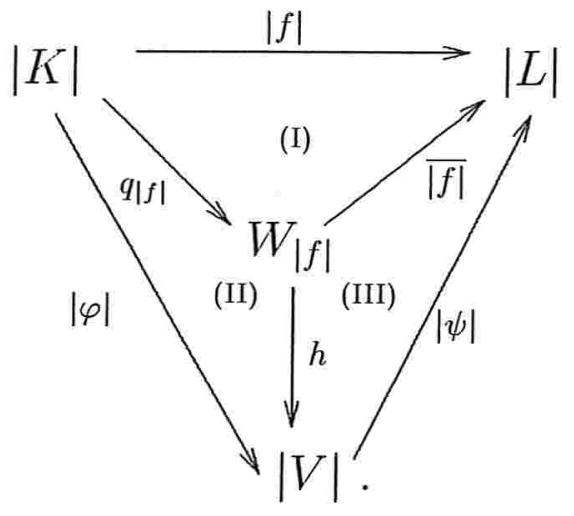

De fato, consideremos primeiramente (II). Seja $x$ um ponto qualquer de $|K| ; \operatorname{temos} h\left(q_{|f|}(x)\right)=|\varphi|\left(q_{|f|}^{-1}\left(q_{|f|}(x)\right)\right)$.

Pelo Lema 3.2.9, sabemos que $|\varphi|\left(q_{|f|}^{-1}\left(q_{|f|}(x)\right)\right)$ é apenas um ponto. Assim, se fixarmos um $z$ arbitrário pertencente a $q_{|f|}^{-1}\left(q_{|f|}(x)\right)$, temos que $|\varphi|\left(q_{|f|}^{-1}\left(q_{|f|}(x)\right)\right)=|\varphi|(z)$. Em particular, $x \in q_{|f|}^{-1}\left(q_{|f|}(x)\right)$, e dessa forma, $|\varphi|\left(q_{|f|}^{-1}\left(q_{|f|}(x)\right)\right)=|\varphi|(x)$. Portanto, $h\left(q_{|f|}(x)\right)=|\varphi|(x), \forall x \in|K|$, ou seja, (II) é comutativo.

Consideremos agora a comutatividade de (III). Se $w$ é um ponto qualquer de $W_{|f|}$, sabendo que $|\psi| \circ|\varphi|=|f|$ e que $\overline{|f|} \circ q_{|f|}=|f|$, podemos concluir que $|\psi| \circ|\varphi|=\overline{|f|} \circ q_{|f|}$. Segue então que

$$
\begin{aligned}
(|\psi| \circ h)(w) & =|\psi|(h(w)) \\
& =|\psi|\left(|\varphi|\left(q_{|f|}^{-1}(w)\right)\right) \\
& =(|\psi| \circ|\varphi|)\left(q_{|f|}^{-1}(w)\right) \\
& \left.=\overline{|f|} \circ q_{|f|}\right)\left(q_{|f|}^{-1}(w)\right) \\
& =\overline{|f|}(w),
\end{aligned}
$$


onde a última igualdade vale, pois $q_{|f|}$ é sobrejetora. Portanto, (III) também é comutativo.

Agora estamos aptos para mostrar o seguinte lema.

Lema 3.2.10 Nas hipóteses acima temos que $k$ é a inversa de $h$, isto é, $k \circ h=\mathrm{id}_{W_{|f|}} e h \circ k=\mathrm{id}_{|V|}$.

\section{Demonstração:}

Seja $w$ um ponto arbitrário de $W_{|f|}$. Temos:

$$
\begin{aligned}
(k \circ h)(w) & =k(h(w)) \\
& =k\left(|\varphi|\left(q_{|f|}^{-1}(w)\right)\right) \\
& =q_{|f|}\left(|\varphi|^{-1}\left(|\varphi|\left(q_{|f|}^{-1}(w)\right)\right)\right) .
\end{aligned}
$$

Dos Lemas 3.2 .8 e 3.2.9, temos que $q_{|f|}\left(|\varphi|^{-1}\left(|\varphi|\left(q_{|f|}^{-1}(w)\right)\right)\right)$ é um único ponto. Como $q_{|f|}\left(|\varphi|^{-1}\left(|\varphi|\left(q_{|f|}^{-1}(w)\right)\right)\right)$ contém $w$, pois $q_{|f|}$ é sobrejetora, este ponto é o próprio $w$ e assim temos $q_{|f|}\left(|\varphi|^{-1}\left(|\varphi|\left(q_{|f|}^{-1}(w)\right)\right)\right)=w$. Em resumo, obtemos $k \circ h(w)=w, \forall w \in W_{|f|}$. Logo, $k \circ h=\mathrm{id}_{W_{|f|}}$.

Consideremos agora $y$ um ponto qualquer de $|V|$. Temos:

$$
\begin{aligned}
(h \circ k)(y) & =h(k(y)) \\
& =h\left(q_{|f|}\left(|\varphi|^{-1}(y)\right)\right) \\
& =|\varphi|\left(q_{|f|}^{-1}\left(q_{|f|}\left(|\varphi|^{-1}(y)\right)\right)\right) .
\end{aligned}
$$

Pelos Lemas 3.2 .8 e 3.2.9, temos que $|\varphi|\left(q_{|f|}^{-1}\left(q_{|f|}\left(|\varphi|^{-1}(y)\right)\right)\right)$ é apenas um ponto de $|V|$. Mas $|\varphi|\left(q_{|f|}^{-1}\left(q_{|f|}\left(|\varphi|^{-1}(y)\right)\right)\right)$ contém $y$, pois $|\varphi|$ é sobrejetora, portanto este ponto é o próprio $y$. Concluímos assim que $(h \circ k)(y)=y, \forall y \in$ $|V|$. Logo, $k$ é a inversa de $h$.

Mostraremos agora que a bijeção $h$, acima definida, é um homeomorfismo. 
Lema 3.2.11 Com as hipóteses acima, $h$ é contínua.

\section{Demonstração:}

Se $B \subset|V|$ é um aberto de $|V|$, então considerando em $W_{|f|}$ a topologia quociente, $h^{-1}(B)$ será um aberto de $W_{|f|}$ se $q_{|f|}^{-1}\left(h^{-1}(B)\right)$ for um aberto de $|K|$. Mas $q_{|f|}^{-1}\left(h^{-1}(B)\right)=\left(h \circ q_{|f|}\right)^{-1}(B)=|\varphi|^{-1}(B)$ é um aberto de $|K|$, pois $|\varphi|$ é contínua. Portanto, $h$ é contínua.

Agora, para mostrarmos que $k$ também é contínua, necessitaremos do seguinte lema.

Lema 3.2.12 Suponhamos $|f|$ uma aplicação própria e $K$ e $L$ são localmente finitos. Então vale o seguinte:

(1) $|\varphi|$ é própria.

(2) $|\varphi|$ é uma aplicação fechada.

\section{Demonstração:}

(1) Seja $D \subset|V|$ um subconjunto compacto de $|V|$, vamos mostrar que $|\varphi|^{-1}(D)$ é um subconjunto compacto de $|K|$.

Considerando a comutatividade do diagrama (3.8), temos que $|\varphi|^{-1}(D) \subset$ $|f|^{-1}(|\psi|(D))$. Como $|\psi|$ é contínua e $|f|$ é uma aplicação própria, temos que $|f|^{-1}(|\psi|(D))$ é um subconjunto compacto de $|K|$. Mas $|\varphi|^{-1}(D)$ é um subconjunto fechado ( $|\varphi|$ é contínua) e está contido no compacto $|f|^{-1}(|\psi|(D))$. Portanto, $|\varphi|^{-1}(D)$ é compacto e, logo, $|\varphi|$ é própria.

(2) Vamos primeiro mostrar que $V$ é localmente finito. Seja $[v]$ um vértice qualquer de $\mathcal{V}$. Se um simplexo $\left\{\left[v_{0}\right], \ldots,\left[v_{m}\right]\right\}$ de $\mathcal{V}$ tem $[v]$ como um de seus vértices, então existem $v_{i}^{\prime} \sim v_{i}$, vértices de $K$, tais que 
$\left\langle v_{0}^{\prime} \cdots v_{m}^{\prime}\right\rangle \in K$, e $v \sim v_{i}^{\prime}$ para algum $i$. Como $|f|$ é própria, existe apenas um número finito de vértices $w$ de $K$ tais que $v \sim w$. Também, para cada um dos tais $w$, temos apenas um número finito de simplexos de $K$ que têm $w$ como um de seus vértices, pois $K$ é localmente finito. Por isso, apenas um número finito de simplexos de $\mathcal{V}$ têm $[v]$ como um de seus vértices. Por isso, $V$ é localmente finito.

Então, $|K|$ e $|V|$ são localmente compactos pelo [Mu, Lemma 2.6, pg.11]. Eles são Hausdorff pelo [Mu, Lemma 2.4, pg.10]. Como $|\varphi|$ é própria, segue que $|\varphi|$ é fechada pela [Br, Proposition 11.5, pg.33].

\section{Lema 3.2.13 A aplicação $k$ é contínua.}

\section{Demonstração:}

Como a aplicação $k$ é a inversa de $h$, basta mostrar que $h$ é uma aplicação fechada.

Seja $D \subset W_{|f|}$ um subconjunto fechado de $W_{|f|}$. Como $q_{|f|}$ é contínua, $q_{|f|}^{-1}(D)$ é um subconjunto fechado em $|K|$. Mas, pelo Lema 3.2.12, $|\varphi|$ é uma aplicação fechada; portanto, $|\varphi|\left(q_{|f|}^{-1}(D)\right)$ é fechado em $|V|$. Por outro lado, $|\varphi|\left(q_{|f|}^{-1}(D)\right)=h(D)$, pois $q_{|f|}$ é sobrejetora. Isto mostra que $k$ é contínua.

No teorema abaixo consideramos $\tilde{f}: \tilde{K} \rightarrow \tilde{L}$ uma aplicação simplicial entre complexos simpliciais localmente finitos. Pela Proposição 1.1.19, dada $L$ a subdivisão baricêntrica de $\tilde{L}$, existem $\tilde{K}$ uma subdivisão de $K$ e $f$ : $K \rightarrow L$ uma aplicação simplicial tal que $|\tilde{f}|=|f|$, onde $|\tilde{f}|:|\tilde{K}| \rightarrow|\tilde{L}|$ e $|f|:|K| \rightarrow|L|$.

Definição 3.2.14 Chamamos $f$ definida acima de uma subdivisão de $\tilde{f}$. 
Teorema 3.2.15 Dada $\tilde{f}: \tilde{K} \rightarrow \tilde{L}$ uma aplicação simplicial entre complexos simpliciais localmente finitos tal que $|\tilde{f}|:|\tilde{K}| \rightarrow|\tilde{L}|$ é própria, considere $f: K \rightarrow L$ uma subdivisão de $\tilde{f}$. Então, existem um complexo simplicial abstrato $\mathcal{V}$, uma aplicação simplicial $\varphi: K \rightarrow V$, uma aplicação simplicial não-degenerada $\psi: V \rightarrow L$ e um homeomorfismo $k:|V| \rightarrow W_{|f|}$, onde $V$ é a realização geométrica de $\mathcal{V}$, que fazem os seguintes diagramas comutarem:
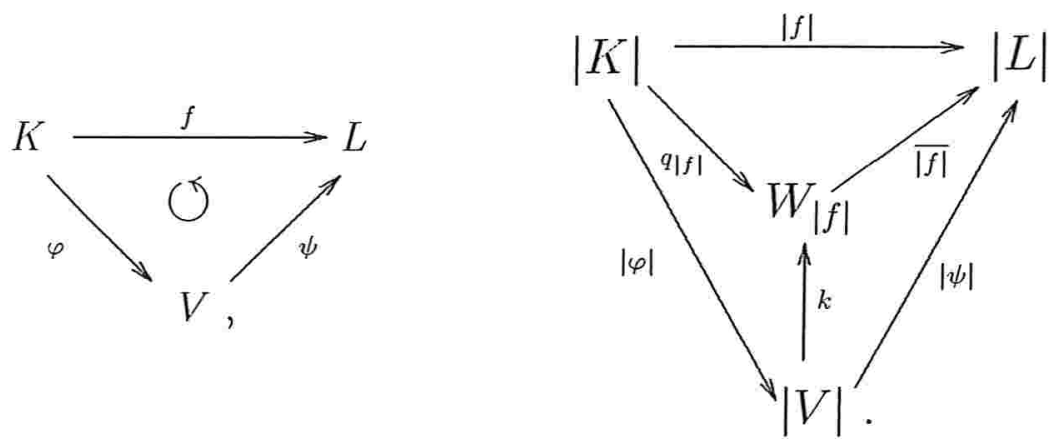

\section{Demonstração:}

Segue dos lemas anteriores.

Observação 3.2.16 O diagrama à esquerda no enunciado do Teorema 3.2.15 pode ser considerado como uma triangulação da fatorização de Stein de $|f|=|\tilde{f}|:|\tilde{K}| \rightarrow|\tilde{L}|$ (Definição 1.3.7).

Observação 3.2.17 O Teorema 3.2 .15 implica que o espaço quociente $W_{|f|}$ é homeomorfo a um poliedro.

Observação 3.2.18 Para uma aplicação simplicial $f$ como definida no Teorema 3.2.15, utilizamos às vezes a seguinte notação: $V=W_{f}, \varphi=q_{f}$ e $\psi=\bar{f}$. 


\subsection{Demonstração do Teorema 1.3 .8}

Seja $g: N \rightarrow P$ uma aplicação contínua entre espaços topológicos $N$ e $P$. Suponhamos que $N$ e $P$ são localmente compactos, $g$ é própria e triangulável. Seja $\tilde{f}: \tilde{K} \rightarrow \tilde{L}$ uma triangulação de $g$, isto é, existem homeomorfismos $\lambda:|\tilde{K}| \rightarrow N$ e $\mu:|\tilde{L}| \rightarrow P$ tais que o diagrama

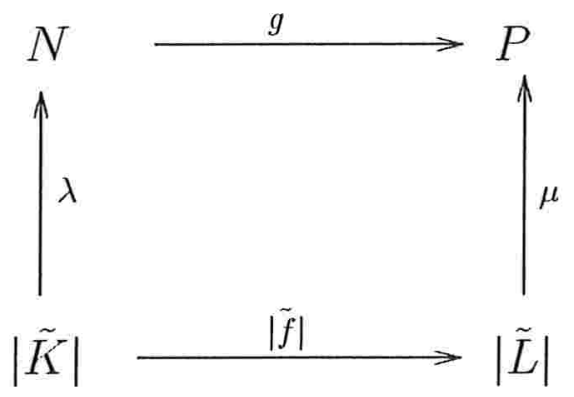

comuta. Note que, pelo [Mu, Lemma 2.6, pg.11], $\tilde{K}$ e $\tilde{L}$ são localmente finitos. Seja $f: K \rightarrow L$ uma subdivisão de $\tilde{f}$ como na Definição 3.2.14. Pelo Lema 1.1.18, $K$ e $L$ também são localmente finitos. A aplicação $|f|:|K| \rightarrow$ $|L|$ é própria e o diagrama

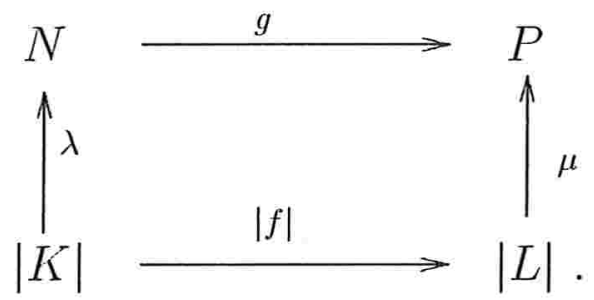

é comutativo, pois $|f|=|\tilde{f}|$.

Consideremos a fatorização de Stein de $|f|$ :

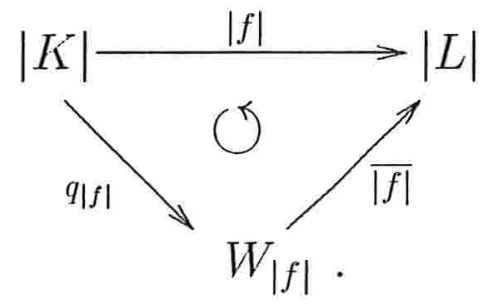


Seja $\Lambda: W_{|f|} \rightarrow W_{g}$ a aplicação definida por $\Lambda(x)=q_{g}\left(\lambda\left(q_{|f|}^{-1}(x)\right)\right)$, para $x \in$ $W_{|f|}$, que faz o seguinte diagrama comutar:

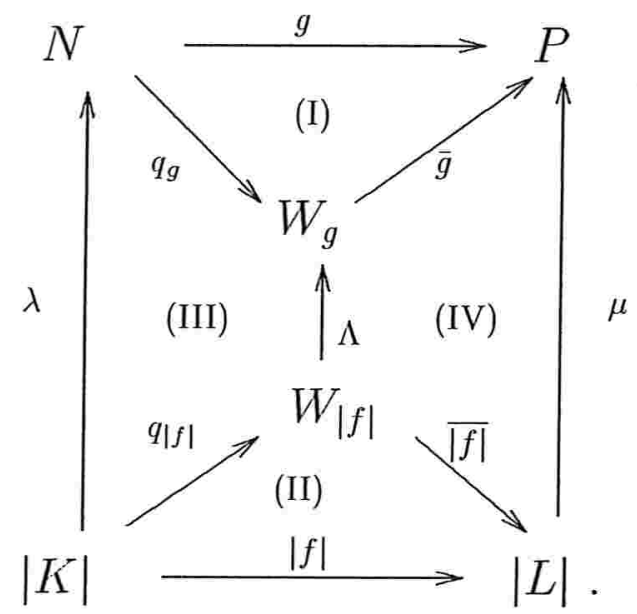

A aplicação $\Lambda$ está bem definida. De fato, como $x \in W_{|f|}$, temos que a imagem inversa $q_{|f|}^{-1}(x)$ é uma componente conexa da fibra $|f|^{-1}(\overline{|f|}(x))$ e, como $\lambda$ é um homeomorfismo, $\lambda\left(q_{|f|}^{-1}(x)\right)$ é uma componente conexa da fibra $g^{-1}(\mu(\overline{|f|}(x)))$. Portanto, pela definição de $q_{g}$, temos que $q_{g}\left(\lambda\left(q_{|f|}^{-1}(x)\right)\right)$ é um único ponto de $W_{g}$.

O diagrama (3.13) é comutativo, pois (I) e (II) são as fatorizações de Stein de $g$ e de $|f|$, respectivamente; e (III) é comutativo pela própria definição de $\Lambda$. Resta-nos verificar a comutatividade de (IV), ou seja, que $\bar{g} \circ \Lambda(x)=$ $\mu \circ \overline{|f|}(x)$, para todo ponto $x$ de $W_{|f|}$.

Seja $x \in W_{|f|}$, então:

$$
\begin{aligned}
\bar{g}(\Lambda(x)) & =\bar{g}\left(q_{g}\left(\lambda\left(q_{|f|}^{-1}(x)\right)\right)\right) \\
& =g\left(\lambda\left(q_{|f|}^{-1}(x)\right)\right) \\
& =\mu\left(|f|\left(q_{|f|}^{-1}(x)\right)\right) \\
& =\mu\left(\overline{|f|}\left(q_{|f|}\left(q_{|f|}^{-1}(x)\right)\right)\right) \\
& =\mu(\overline{|f|}(x)),
\end{aligned}
$$


e as igualdades acima valem:

(3.14) usando a definição de $\Lambda$;

(3.15) $\bar{g} \circ q_{g}=g$, pela comutatividade de (I);

(3.16) $g \circ \lambda=\mu \circ|f|$, pela comutatividade de (3.11);

(3.17) $|f|=\overline{|f|} \circ q_{|f|}$, pela comutatividade de (II);

(3.18) $q_{|f|}\left(q_{|f|}^{-1}(x)\right)=x, \forall x \in W_{|f|}$, pois $q_{|f|}$ é sobrejetora.

Portanto, (IV) é comutativo.

Seja $U \subset W_{g}$ um aberto qualquer. Então, $\Lambda^{-1}(U)$ é aberto de $W_{|f|}$ se, e somente se, $q_{|f|}^{-1}\left(\Lambda^{-1}(U)\right)$ é um aberto de $|K|$. Mas, pela comutatividade (III) do diagrama (3.13), temos que $q_{|f|}^{-1}\left(\Lambda^{-1}(U)\right)=\lambda^{-1}\left(q_{g}^{-1}(U)\right)$, o qual é um aberto de $|K|$, pois $q_{g}$ e $\lambda$ são contínuas. Por isso, $\Lambda^{-1}(U)$ é aberto de $W_{|f|}$, e $\Lambda$ é contínua.

Definimos agora a aplicação $\Gamma: W_{g} \rightarrow W_{|f|}$ da seguinte forma:

$$
\Gamma(y)=q_{|f|}\left(\lambda^{-1}\left(q_{g}^{-1}(y)\right)\right), \text { para } y \in W_{g} .
$$

Podemos mostrar que $\Gamma$ está bem definida usando um argumento similar ao de $\Lambda$.

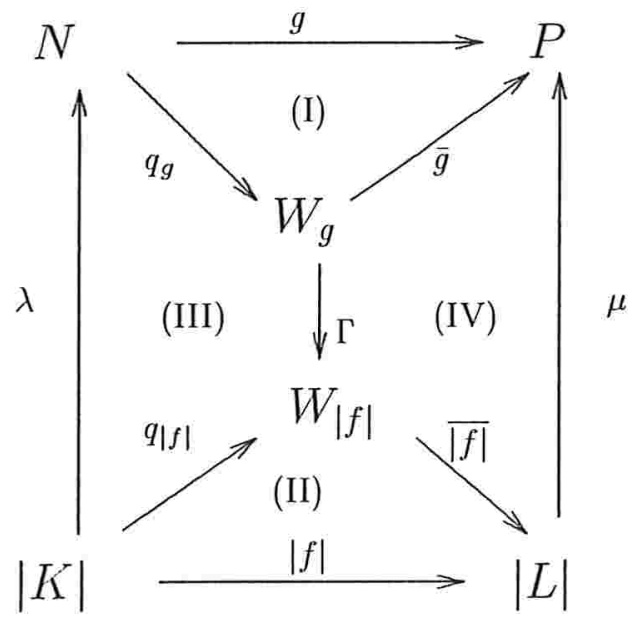


A aplicação $\Gamma$ faz o diagrama (3.19) comutar. Basta verificar a comutatividade de (IV). De fato, seja $y \in W_{g}$ e consideremos a composta $\mu \circ \overline{|f|} \circ \Gamma$ :

$$
\begin{aligned}
\mu(\overline{|f|}(\Gamma(y))) & =\mu\left(\overline{|f|}\left(q_{|f|}\left(\lambda^{-1}\left(q_{g}^{-1}(y)\right)\right)\right)\right) \\
& =\mu\left(|f|\left(\lambda^{-1}\left(q_{g}^{-1}(y)\right)\right)\right) \\
& =g\left(\lambda\left(\lambda^{-1}\left(q_{g}^{-1}(y)\right)\right)\right) \\
& =g\left(q_{g}^{-1}(y)\right) \\
& =\bar{g}(y),
\end{aligned}
$$

e as igualdades acima valem:

(3.20) usando a definição de $\Gamma$;

(3.21) $\overline{|f|} \circ q_{|f|}=|f|$, pela comutatividade de (II);

(3.22) $\mu \circ|f|=g \circ \lambda$, pela comutatividade de (3.11);

(3.23) $\lambda$ é um homeomorfismo;

(3.24) $q_{g}^{-1}(y)$ é uma componente conexa de $g^{-1}(\bar{g}(y))$.

Portanto, (IV) é comutativo.

Seja $U \subset W_{|f|}$ um aberto qualquer. Então, $\Gamma^{-1}(U)$ é aberto de $W_{g}$ se, e somente se, $q_{g}^{-1}\left(\Gamma^{-1}(U)\right)$ é um aberto de $N$. Mas, pela comutatividade (III) do diagrama (3.19), temos que $q_{g}^{-1}\left(\Gamma^{-1}(U)\right)=\lambda\left(q_{|f|}^{-1}(U)\right)$, o qual é um aberto de $N$, pois $q_{|f|}$ e $\lambda^{-1}$ são contínuas. Por isso, $\Gamma^{-1}(U)$ é aberto de $W_{g}$, e $\Gamma$ é contínua.

A aplicação $\Gamma$ é a inversa de $\Lambda$. De fato, seja $x \in W_{|f|}$ e consideremos a composta

$$
\Gamma(\Lambda(x))=q_{|f|}\left(\lambda^{-1}\left(q_{g}^{-1}\left(q_{g}\left(\lambda\left(q_{|f|}^{-1}(x)\right)\right)\right)\right)\right) .
$$

Seja $A=\lambda\left(q_{|f|}^{-1}(x)\right)$. Como $\lambda$ é um homeomorfismo, temos que $A$ é uma componente conexa inteira de uma fibra de $g$, ou seja, $q_{g}^{-1}\left(q_{g}(A)\right)=A$. 
Segue que $\Gamma(\Lambda(x))=q_{|f|}\left(\lambda^{-1}\left(\lambda\left(q_{|f|}^{-1}(x)\right)\right)\right)$. Como $\lambda$ é um homeomorfismo, $\Gamma(\Lambda(x))=q_{|f|}\left(q_{|f|}^{-1}(x)\right)$. Mas, $q_{|f|}\left(q_{|f|}^{-1}(x)\right)=x$, pois $q_{|f|}$ é sobrejetora, e isto implica que $\Gamma(\Lambda(x))=x$.

Analogamente mostramos que $\Lambda(\Gamma(y))=y$, para qualquer $y \in W_{g}$. Logo, $\Lambda$ é um homeomorfismo.

Consideremos agora $\mathcal{V}$ o complexo simplicial abstrato, $V$ a sua realização geométrica, $\varphi: K \rightarrow V, \psi: V \rightarrow L$ e $k:|V| \rightarrow W_{|f|}$ as aplicações dadas pelo Teorema 3.2.15.

Os diagramas comutativos (3.9) e (3.13) nos fornecem o seguinte diagrama também comutativo:

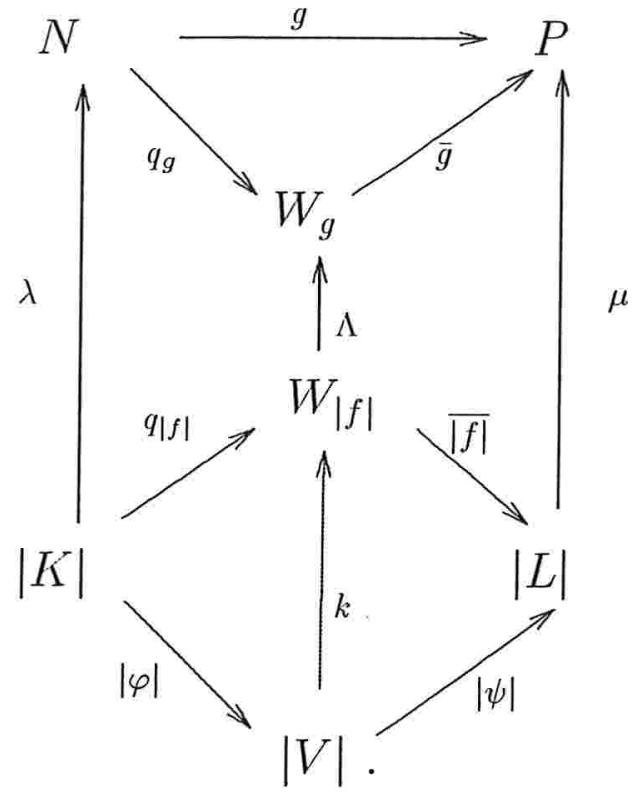

Então a aplicação $\Theta:|V| \rightarrow W_{g}$ definida por $\Theta=\Lambda \circ k$ é um homeomorfismo que satisfaz o diagrama comutativo da Definição 1.3.7. Por isso, temos que

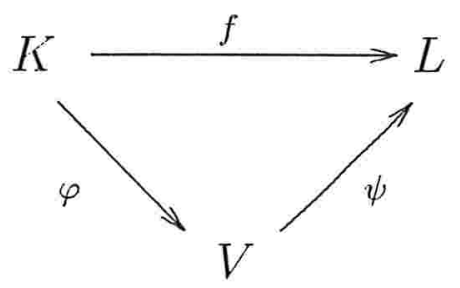


é uma triangulação da fatorização de Stein de $g$. Isto completa a demonstração do Teorema 1.3.8 


\section{Capítulo 4}

\section{Característica de Euler}

O objetivo principal dos capítulos subseqüentes é relacionar o número de singularidades de aplicações $\mathcal{C}^{\infty}$-estáveis, $g: N^{n} \rightarrow \mathbb{R}^{p}$, com as características de Euler de subconjuntos de $N^{n}, g\left(N^{n}\right)$ e $W_{g}$. Nas duas primeiras seções deste capítulo definimos a característica usual de Euler e trabalhamos com a fórmula demonstrada por Ballesteros \& Saeki em [BS] sobre o diagrama de Stein de uma aplicação simplicial $f: X \rightarrow Y$ entre complexos simpliciais. Também consideramos, em alguns casos, conjuntos não fechados e, assim, não podemos utilizar a característica de Euler usual. Para contornar esta situação, introduzimos, no final deste capítulo, o conceito de característica combinatória de Euler.

Por comodidade, nos Capítulos 4, 5 e 6, não faremos distinção entre as notações de complexos simpliciais geométricos e de seus espaços subjacentes, ou seja, usaremos a notação $f: K \rightarrow L$ no lugar de $|f|:|K| \rightarrow|L|$.

\subsection{Característica de Euler}

A seguir definimos a característica de Euler usual de um complexo simplicial e de seu espaço subjacente. 
Definição 4.1.1 Dado um complexo simplicial finito $K$ de dimensão $m$, seja $\alpha_{q}$ o número de $q$-simplexos em $K$, para cada $q \geq 0$. A característica de Euler de $K$ denotado por $\chi(K)$, é definida por:

$$
\chi(K)=\sum_{q=0}^{m}(-1)^{q} \alpha_{q} .
$$

Definição 4.1.2 Dado um complexo simplicial $K$ de dimensão $m$, definimos a característica de Euler de $|K|$ como sendo

$$
\chi(|K|)=\sum_{q=0}^{m}(-1)^{q} a_{q}
$$

onde $a_{q}$ é o número de $q$-simplexos em $K$, para cada $q \geq 0$. Note que os números $\alpha_{q}$ dependem da triangulação do poliedro $|K|$, mas é bem conhecido o fato de que a característica de Euler $\chi(|K|)$ de $|K|$ não depende da escolha da triangulação.

A relação entre as definições acima e o número de Betti é dada pelo teorema abaixo.

Teorema 4.1.3 Se K é um complexo simplicial finito de dimensão m, então

$$
\chi(K)=\sum_{q=0}^{m}(-1)^{q} \operatorname{rank} H_{q}(K ; \mathbb{Z}) .
$$

\section{Demonstração:}

Ver [Ro, Theorem 7.15, pg.146].

Observação 4.1.4 A característica de Euler de $K$ é um invariante topológico de $|K|[\mathrm{Mu}, \mathrm{pg} .124]$. 
Exemplo 4.1.5 Segue abaixo alguns exemplos de características de Euler.

$$
\begin{aligned}
\chi\left(S^{n}\right) & =1+(-1)^{n}, \\
\chi\left(S^{1} \times S^{1}\right) & =1-2+1=0, \\
\chi\left(\mathbb{I R P} P^{n}\right) & =\frac{1}{2}\left(1+(-1)^{n}\right), \\
\chi\left(\mathbb{C} P^{n}\right) & =n+1 .
\end{aligned}
$$

A característica de Euler é uma ferramenta muito importante dentro da Topologia, por exemplo, podemos classificar superfícies fechadas usando a característica de Euler.

\section{2 Índices locais para aplicações simpliciais}

Em [BS], Ballesteros \& Saeki definem índices locais para aplicações simpliciais $f: K \rightarrow L$, onde $K$ é um complexo simplicial finito, e demonstram uma fórmula relacionando tais índices com as características de Euler do domínio e da imagem de $f$. Nesta seção, damos um refinamento desta fórmula através da fatorização de Stein de $f$.

Definição 4.2.1 Dada uma aplicação simplicial, $f: X \rightarrow Y$, onde $X$ é um complexo simplicial finito:

(i) o conjunto dos pontos de auto-intersecção de $f$, denotado por $D(f)$, é o fecho do conjunto $\left\{x \in X: f^{-1}(f(x)) \neq\{x\}\right\}$ em $X$;

(ii) $M(f)=f(D(f))$ é o conjunto dos pontos múltiplos de $f$.

Observação 4.2.2 Se $v \in M(f)$, então $f^{-1}(v) \subset D(f)$. Portanto, $w \in$ $\left(f^{-1}(v)\right)^{(0)}$ pertence a $D(f)$.

Podemos definir, segundo [BS], um índice para os vértices $v$ de $M(f)$ da seguinte forma:

$$
\operatorname{Ind}_{f}(v)=\chi_{v}(f(X))-\sum_{w \in\left(f^{-1}(v)\right)^{(0)}} \chi_{w}(X),
$$


onde $\chi_{v}(f(X))=\sum_{\tau \ni v} \frac{(-1)^{\operatorname{dim} \tau}}{\operatorname{dim} \tau+1}$ e $\tau$ é tomado no conjunto de todos os simplexos de $f(X)$ que contém $v, \chi_{w}(X)=\sum_{\sigma \ni w} \frac{(-1)^{\operatorname{dim} \sigma}}{\operatorname{dim} \sigma+1}$ e $\sigma$ é tomado no conjunto de todos os simplexos de $X$ que contém $w$, e $\left(f^{-1}(v)\right)^{(0)}$ é o conjunto dos vértices de $f^{-1}(v)$. Ou seja,

$$
\operatorname{Ind}_{f}(v)=\sum_{\tau \ni v} \frac{(-1)^{\operatorname{dim} \tau}}{\operatorname{dim} \tau+1}-\sum_{w \in\left(f^{-1}(v)\right)^{(0)}} \sum_{\sigma \ni w} \frac{(-1)^{\operatorname{dim} \sigma}}{\operatorname{dim} \sigma+1} .
$$

Observação 4.2.3 Observemos que podemos definir o índice para qualquer vértice $v \in(f(X))^{(0)}$ usando a equação (4.1) e, segundo a definição acima, temos $\operatorname{Ind}_{f}(v)=0$ para qualquer $v \in(f(X))^{(0)}-M(f)^{(0)}$. Também, se $v \in M(f)^{(0)}$, então temos que

$$
\operatorname{Ind}_{f}(v)=\chi_{v}(M(f))-\sum_{w \in\left(f^{-1}(v)\right)^{(0)}} \chi_{w}(D(f))
$$

onde $\chi_{v}(M(f))=\sum_{\tau \ni v} \frac{(-1)^{\operatorname{dim} \tau}}{\operatorname{dim} \tau+1}$ e $\tau$ é tomado no conjunto de todos os simplexos de $M(f)$ que contém $v, \chi_{w}(D(f))=\sum_{\sigma \ni w} \frac{(-1)^{\operatorname{dim} \sigma}}{\operatorname{dim} \sigma+1}$ e $\sigma$ é tomado no conjunto de todos os simplexos de $D(f)$ que contém $w$ (veja a Observação 4.2.2).

Segue, ainda, de [BS, Theorem 3.3] o seguinte resultado:

Teorema 4.2.4 Seja $f: X \rightarrow Y$ uma aplicação simplicial entre dois complexos simpliciais, onde $X$ é finito. Então,

$$
\chi(f(X))-\chi(X)=\sum_{v \in(f(X))^{(0)}} \operatorname{Ind}_{f}(v)
$$

com $(f(X))^{(0)}$ denotando o conjunto dos vértices de $f(X)$.

Conforme o Teorema 3.2.15 e utilizando as notações da Observação 3.2.18, consideremos a seguinte fatorização de Stein para uma aplicação simplicial 
$f: X \rightarrow Y$ entre complexos simpliciais, onde $X$ é finito, $W_{f}$ é um complexo simplicial, $q_{f}$ e $\bar{f}$ são aplicações simpliciais:

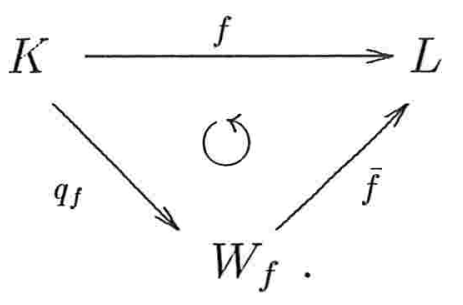

Caso necessário, consideramos ainda uma subdivisão de $f$ conforme a Definição 3.2.14. Notemos que $f: X \rightarrow Y$ é própria, pois $X$ é um complexo simplicial finito e o seu espaço subjacente é compacto.

Como $X$ é finito, segue que $W_{f}$ também é finito; portanto, podemos aplicar o [BS, Theorem 3.3] acima enunciado para as aplicações $f, q_{f}$ e $\bar{f}$, obtendo o seguinte:

$$
\begin{aligned}
\chi(f(X))-\chi(X) & =\sum_{v \in f(X)^{(0)}} \operatorname{Ind}_{f}(v), \\
\chi\left(\bar{f}\left(W_{f}\right)\right)-\chi\left(W_{f}\right) & =\sum_{v \in \bar{f}\left(W_{f}\right)^{(0)}} \operatorname{Ind}_{\bar{f}}(v), \\
\chi\left(q_{f}(X)\right)-\chi(X) & =\sum_{u \in q_{f}(X)^{(0)}} \operatorname{Ind}_{q_{f}}(u) .
\end{aligned}
$$

Como $f(X)=\bar{f}\left(W_{f}\right)$ e $q_{f}(X)=W_{f}$, segue que:

$$
\begin{aligned}
\chi(f(X))-\chi(X) & =\sum_{v \in f(X)^{(0)}} \operatorname{Ind}_{f}(v), \\
\chi(f(X))-\chi\left(W_{f}\right) & =\sum_{v \in f(X)^{(0)}} \operatorname{Ind}_{\bar{f}}(v), \\
\chi\left(W_{f}\right)-\chi(X) & =\sum_{u \in W_{f}^{(0)}} \operatorname{Ind}_{q_{f}}(u) .
\end{aligned}
$$


Da equação:

$$
\chi(f(X))-\chi(X)=\chi(f(X))+\left(-\chi\left(W_{f}\right)+\chi\left(W_{f}\right)\right)-\chi(X)
$$

segue que:

$$
\sum_{v \in f(X)^{(0)}} \operatorname{Ind}_{f}(v)=\sum_{v \in f(X)^{(0)}} \operatorname{Ind}_{\bar{f}}(v)+\sum_{u \in W_{f}^{(0)}} \operatorname{Ind}_{q_{f}}(u) .
$$

Assim, a fatorização de Stein de $f$ decompõe o somatório dos índices de $f$, definido em [BS], em dois novos somatórios; e isto leva-nos a estabelecer o seguinte:

Lema 4.2.5 Dada uma aplicação simplicial $f: X \rightarrow Y$ entre complexos simpliciais, onde $X$ é finito, para cada $v \in f(X)$, temos:

$$
\operatorname{Ind}_{f}(v)=\operatorname{Ind}_{\bar{f}}(v)+\sum_{u \in\left(\bar{f}^{-1}(v)\right)^{(0)}} \operatorname{Ind}_{q_{f}}(v) .
$$

\section{Demonstração:}

\section{Sejam}

$$
\begin{aligned}
& \operatorname{Ind}_{f}(v)=\sum_{\sigma \ni v} \frac{(-1)^{\operatorname{dim} \sigma}}{\operatorname{dim} \sigma+1}-\sum_{w \in\left(f^{-1}(v)\right)^{(0)}} \sum_{\tau \ni w} \frac{(-1)^{\operatorname{dim} \tau}}{\operatorname{dim} \tau+1}, \text { onde } \sigma \subset f(X), \tau \subset X ; \\
& \operatorname{Ind}_{\bar{f}}(v)=\sum_{\sigma \ni v} \frac{(-1)^{\operatorname{dim} \sigma}}{\operatorname{dim} \sigma+1}-\sum_{u \in\left(\bar{f}^{-1}(v)\right)^{(0)}} \sum_{\delta \ni u} \frac{(-1)^{\operatorname{dim} \delta}}{\operatorname{dim} \delta+1}, \text { onde } \sigma \subset \bar{f}\left(W_{f}\right), \delta \subset W_{f} ; \\
& \operatorname{Ind}_{q_{f}}(u)=\sum_{\delta \ni u} \frac{(-1)^{\operatorname{dim} \delta}}{\operatorname{dim} \delta+1}-\sum_{w \in\left(q_{f}^{-1}(u)\right)^{(0)}} \sum_{\tau \ni w} \frac{(-1)^{\operatorname{dim} \tau}}{\operatorname{dim} \tau+1}, \text { onde } \delta \subset q_{f}(X), \tau \subset X .
\end{aligned}
$$

Primeiramente observemos que $f(X)=\bar{f}\left(W_{f}\right)$; assim: 


$$
\begin{aligned}
& \operatorname{Ind}_{f}(v)=\sum_{\sigma \ni v} \frac{(-1)^{\operatorname{dim} \sigma}}{\operatorname{dim} \sigma+1}-\sum_{w \in\left(f^{-1}(v)\right)^{(0)}} \sum_{\tau \ni w} \frac{(-1)^{\operatorname{dim} \tau}}{\operatorname{dim} \tau+1} \\
& =\sum_{\sigma \ni v} \frac{(-1)^{\operatorname{dim} \sigma}}{\operatorname{dim} \sigma+1}-\sum_{u \in\left(\bar{f}^{-1}(v)\right)^{(0)}} \sum_{\delta \ni u} \frac{(-1)^{\operatorname{dim} \delta}}{\operatorname{dim} \delta+1}+\sum_{u \in\left(\bar{f}^{-1}(v)\right)^{(0)}} \sum_{\delta \ni u} \frac{(-1)^{\operatorname{dim} \delta}}{\operatorname{dim} \delta+1} \\
& -\sum_{w \in\left(f^{-1}(v)\right)^{(0)}} \sum_{\tau \ni w} \frac{(-1)^{\operatorname{dim} \tau}}{\operatorname{dim} \tau+1} \\
& =\operatorname{Ind}_{\bar{f}}(v)+\sum_{u \in\left(\bar{f}^{-1}(v)\right)^{(0)}} \sum_{\delta \ni u} \frac{(-1)^{\operatorname{dim} \delta}}{\operatorname{dim} \delta+1}-\sum_{u \in\left(\bar{f}^{-1}(v)\right)^{(0)}} \sum_{w \in\left(q_{f}^{-1}(u)\right)^{(0)}} \sum_{\tau \ni w} \frac{(-1) \operatorname{dim} \tau}{\operatorname{dim} \tau+1} \\
& =\operatorname{Ind}_{\bar{f}}(v)+\sum_{u \in\left(\bar{f}^{-1}(v)\right)^{(0)}}\left(\sum_{\delta \ni u} \frac{(-1)^{\operatorname{dim} \delta}}{\operatorname{dim} \delta+1}-\sum_{w \in\left(q_{f}^{-1}(u)\right)^{(0)}} \sum_{\tau \ni w} \frac{(-1)^{\operatorname{dim} \tau}}{\operatorname{dim} \tau+1}\right) \\
& =\operatorname{Ind}_{\bar{f}}(v)+\sum_{u \in\left(\bar{f}^{-1}(v)\right)^{(0)}} \operatorname{Ind}_{q_{f}}(u) .
\end{aligned}
$$

Portanto, temos que $\operatorname{Ind}_{f}(v)=\operatorname{Ind}_{\bar{f}}(v)+\sum_{u \in\left(\bar{f}^{-1}(v)\right)^{(0)}} \operatorname{Ind}_{q_{f}}(u)$.

\subsection{Característica combinatória de Euler}

Damos, a seguir, uma definição mais geral da característica de Euler, a qual denominamos de característica combinatória de Euler. Este conceito é fundamental no decorrer dos Capítulos 5 e 6 , onde necessitamos calcular invariantes topológicos de conjuntos não fechados.

Definição 4.3.1 Seja $E$ um espaço topológico homeomorfo a $\mathbb{R}^{n}$, para algum $n$, definimos a característica combinatória de Euler de $E$ por: $\chi_{c}(E)=$ $(-1)^{n}$. Se $E$ é a união disjunta finita de conjuntos $A_{i}$, onde cada $A_{i}$ é homeomorfo a $\mathbb{R}^{n}$, para algum $n$, então $\chi_{c}(E)=\sum_{i} \chi_{c}\left(A_{i}\right)$. 
Observação 4.3.2 A característica combinatória independe da escolha da decomposição $E=\bigcup_{i} A_{i}$ e é um invariante topológico [KP, Proposition 1, pg.21], [Ro, pg.221]. Ainda mais, vale que $\chi_{c}(X \times Y)=\chi_{c}(X) \chi_{c}(Y)[\mathrm{Vi}$, pg.73], [Ro, Theorem 8.45, pg.221].

Exemplo 4.3.3 Vejamos a seguir a característica combinatória em alguns casos:

a) Seja $P$ um ponto, então $\chi_{c}(P)=(-1)^{0}=1$.

$$
+P
$$

b) Seja $J$ um segmento sem os extremos, então $\chi_{c}(J)=(-1)^{1}=-1$.

\section{J}

c) Seja $\sigma$ um 2-simplexo, então $\chi_{c}(\stackrel{\circ}{\sigma})=(-1)^{2}=1$.

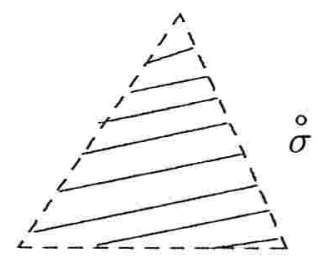

d) Seja $X$ conforme a figura abaixo:

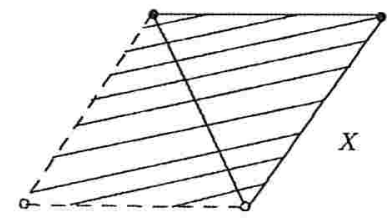

Então, $\chi_{c}(X)=2(-1)^{0}+3(-1)^{1}+2(-1)^{2}=2-3+2=1$. 
Observação 4.3.4 A característica combinatória coincide com a característica usual de Euler para complexos simpliciais.

Exemplo 4.3.5 Considere uma triangulação de $S^{2}$ conforme a figura abaixo:

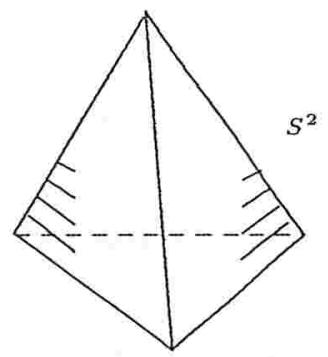

Então, $\chi_{c}\left(S^{2}\right)=4(-1)^{0}+6(-1)^{1}+4(-1)^{2}=4-6+4=2=\chi\left(S^{2}\right)$. 


\section{Capítulo 5}

\section{Fórmulas referentes a aplicações estáveis de uma 3-variedade no $\mathbb{R}^{2}$}

Aplicando o Teorema 1.3.8, obtemos fórmulas que relacionam as singularidades de uma aplicação $\mathcal{C}^{\infty}$-estável, $g: N^{3} \rightarrow \mathbb{R}^{2}$, onde $N^{3}$ é uma variedade fechada e orientável de dimensão 3 , com alguns invariantes topológicos.

Mais especificamente, como $W_{g}$ é um poliedro e $\bar{g}: W_{g} \rightarrow \mathbb{R}^{2}$ é uma aplicação simplicial, aplicamos o Teorema 4.2 .4 a $\bar{g}$.

Assim, utilizando a definição de índice $\operatorname{Ind}_{f}(v)$ dada em [BS], deduzimos uma fórmula que nos permite, por exemplo, concluir que as cúspides de $g$ ocorrem aos pares. Este é um resultado similar àquele obtido por Izumiya \& Marar em [IM1], [IM2] e [IM3].

Por outro lado, damos uma nova definição de índice e com isso obtemos uma segunda fórmula que nos possibilita uma nova demonstração para o seguinte resultado: "A característica de Euler de toda variedade fechada de dimensão três é zero", sem utilizar a dualidade de Poincaré. 


\subsection{Triangulação da fatorização de Stein}

No que segue, fazemos um estudo mais detalhado de uma triangulação da fatorização de Stein de uma aplicação $\mathcal{C}^{\infty}$-estável (veja o Corolário 1.4.7). Primeiramente, enunciamos uma proposição que pode ser encontrada, por exemplo, em [Le] e que caracteriza, local e globalmente, as aplicações estáveis $g: N^{3} \rightarrow \mathbb{R}^{2}$, de variedades de dimensão 3 no plano.

Proposição 5.1.1 Sejam $N^{3}$ uma variedade tridimensional e $g: N^{3} \longrightarrow \mathbb{R}^{2}$ uma aplicação $\mathcal{C}^{\infty}$ e própria. Então g é $\mathcal{C}^{\infty}$-estável se, e somente se, as seguintes condiçôes locais e globais estão satisfeitas:

Para todo $p \in S(g)$, existem coordenadas locais $(x, y, z)$ centradas em $p$, e $(X, Y)$ centradas em $g(p)$ tais que

$$
\text { L1) }\left\{\begin{array}{l}
X \circ g=x, \\
Y \circ g=y^{2}+z^{2} ;
\end{array} \quad(p: \text { ponto de dobra definida })\right.
$$

ou

L2) $\left\{\begin{array}{l}X \circ g=x, \\ Y \circ g=y^{2}-z^{2} ;\end{array} \quad(p:\right.$ ponto de dobra indefinida $)$

ou

$$
\text { L3) }\left\{\begin{array}{l}
X \circ g=x, \\
Y \circ g= \pm z^{2}+y x+y^{3}
\end{array} \quad(p: \text { ponto de cúspide })\right.
$$

$e$

G1) para todo ponto de cúspide $p \in S(g), g^{-1}(g(p)) \cap S(g)=\{p\}$ e

G2) $\left.g\right|_{S(g) \backslash C}$ é uma imersão com cruzamentos normais

onde $C$ é o conjunto dos pontos de cúspides.

Observação 5.1.2 A definição de imersão com cruzamentos normais pode ser encontrada em [GG, Chapter III, Definition 3.1]. 
Sejam $N^{3}$ uma variedade diferenciável orientável e fechada e $g: N^{3} \rightarrow \mathbb{R}^{2}$ uma aplicação diferenciável $\mathcal{C}^{\infty}$-estável. O conjunto singular de $g, S(g)$, é a união do conjunto de pontos de dobra definida $S_{0}$, do conjunto de pontos de dobra indefinida $S_{1}$ e do conjunto $C$ de pontos isolados denominados de cúspides ([KLP], [PF], [KS]).

Consideremos a fatorização de Stein de $g$ :

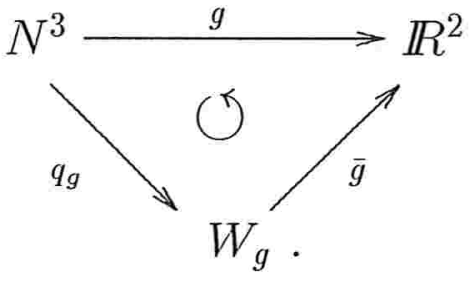

Sabemos que todo ponto $p$ de $W_{g}$ admite uma das seguintes vizinhanças canônicas ([Le], [KLP]):
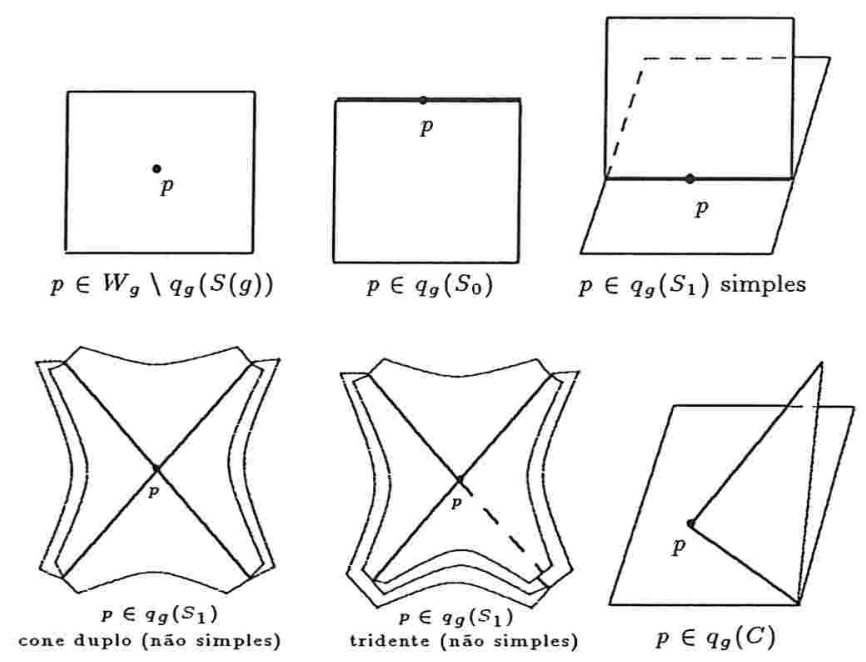

onde o traço mais forte representa a imagem do conjunto singular de $g$ através de $q_{g}$. 
Observação 5.1.3 As imagens das vizinhanças canônicas acima, através de $\bar{g}$, são da forma [Le, pg.20]:
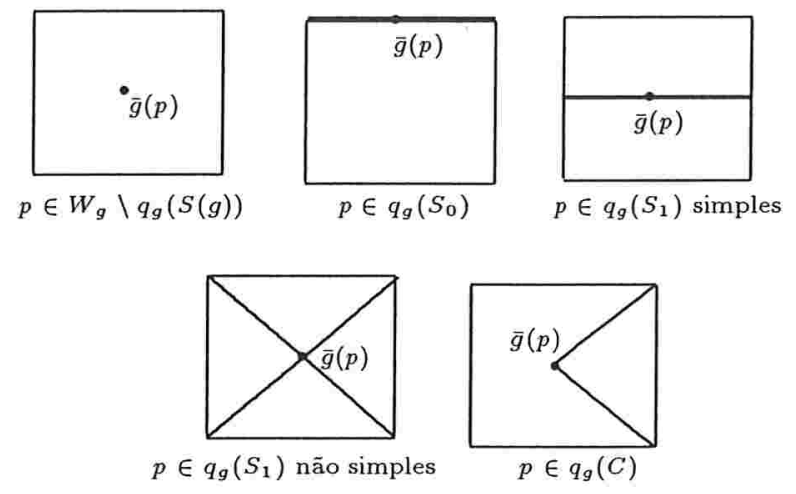

De Verona [Ve1],[Ve2] sabemos que $g$ é triangulável e, do Teorema 1.3.8 ou do Corolário 1.4.7, sabemos que $W_{g}$ é homeomorfo a um poliedro. Sejam $f: K \rightarrow L$ uma triangulação de $g, q_{f}$ e $\bar{f}$ triangulações das aplicações $q_{g}$ e $\bar{g}$, respectivamente, da fatorização de Stein de $g$ :

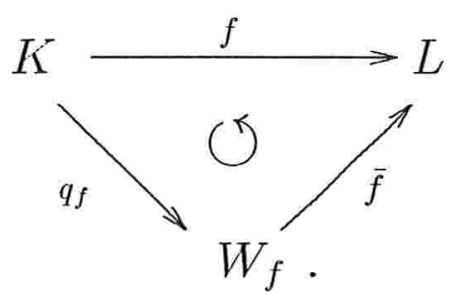

Observação 5.1.4 Seja $r \in \bar{f}\left(W_{f}\right)=f(K)$. Vamos descrever o comportamento do "multi-germ" $\bar{f}: W_{f}, \bar{f}^{-1}(r) \rightarrow L, r$ caso a caso, como a seguir. Denotamos por $S(f), S_{0}, S_{1}$ e $C$ os subconjuntos de $K$ que correspondem a $S(g), S_{0}, S_{1}$ e $C$, respectivamente. 
I) $r \notin f(S(f))$.

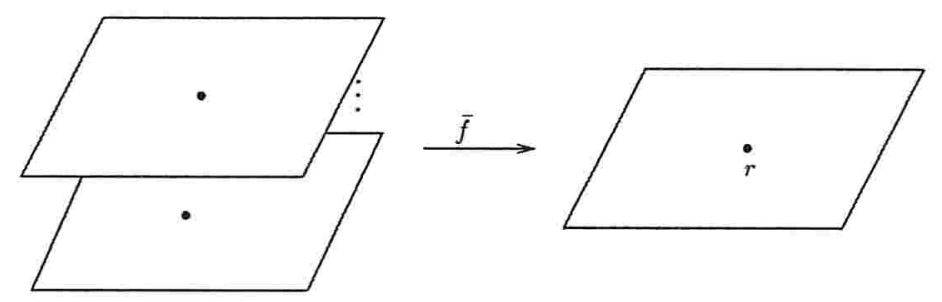

II) $r \in f(S(f))$.

a) $f^{-1}(r) \cap S(f)=f^{-1}(r) \cap S_{0}=\{p\}$.

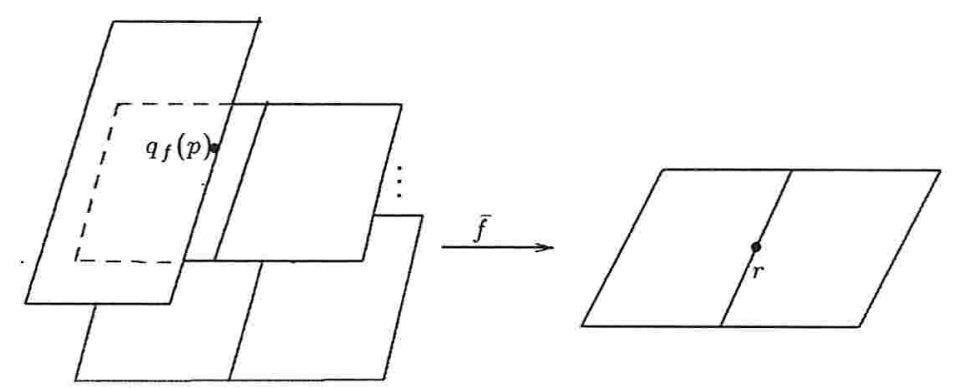

b) $f^{-1}(r) \cap S(f)=f^{-1}(r) \cap S_{1}=\{p\}$.
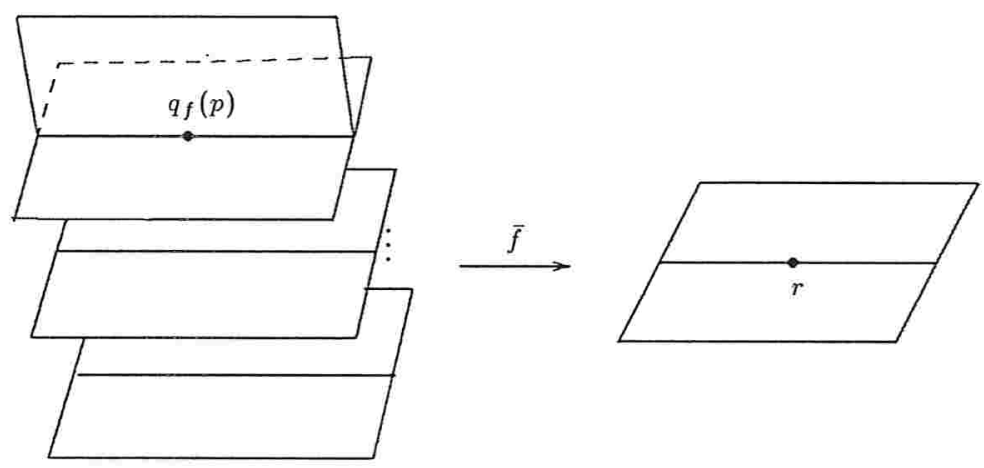
c) $f^{-1}(r) \cap S(f)=f^{-1}(r) \cap S_{0}=\left\{p, p^{\prime}\right\}$.
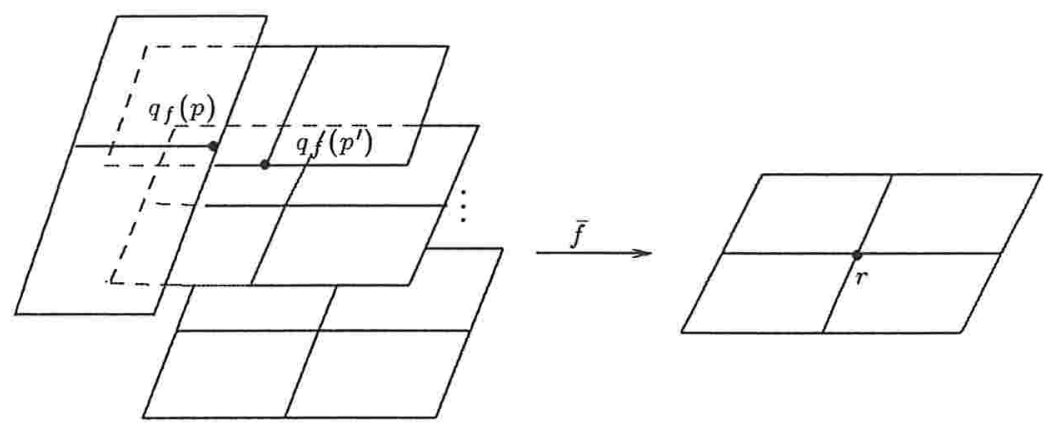

d) $f^{-1}(r) \cap S_{0}=\{p\}$ e $f^{-1}(r) \cap S_{1}=\left\{p^{\prime}\right\}$.

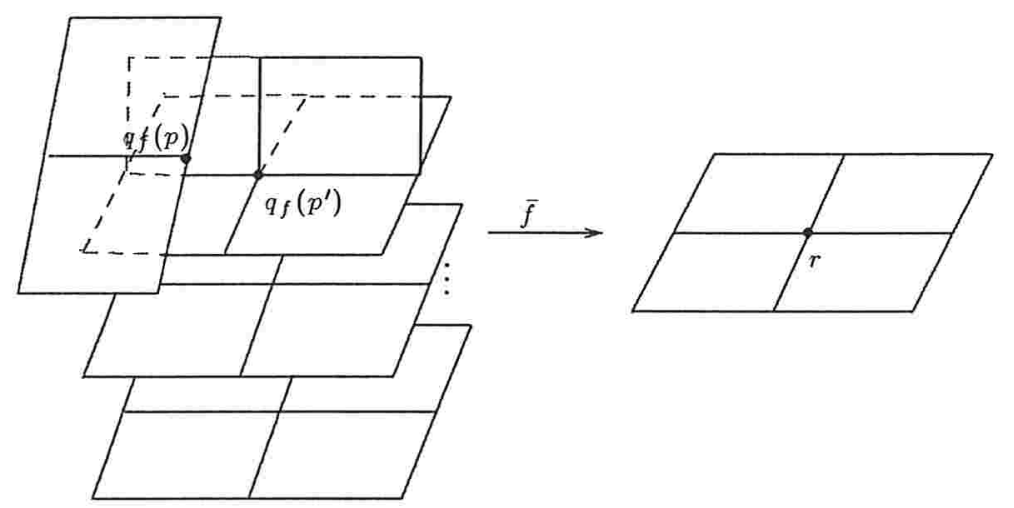

e) $f^{-1}(r) \cap S(f)=f^{-1}(r) \cap S_{1}=\left\{p, p^{\prime}\right\}$ e $q_{f}(p) \neq q_{f}\left(p^{\prime}\right)$.
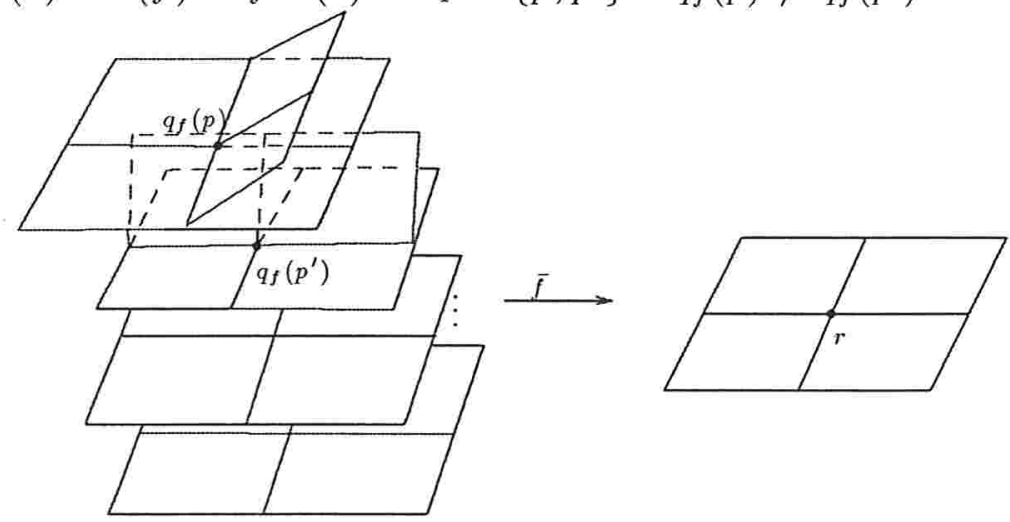
f) $f^{-1}(r) \cap S(f)=f^{-1}(r) \cap S_{1}=\left\{p, p^{\prime}\right\}$ e $q_{f}(p)=q_{f}\left(p^{\prime}\right)$ é um cone duplo.

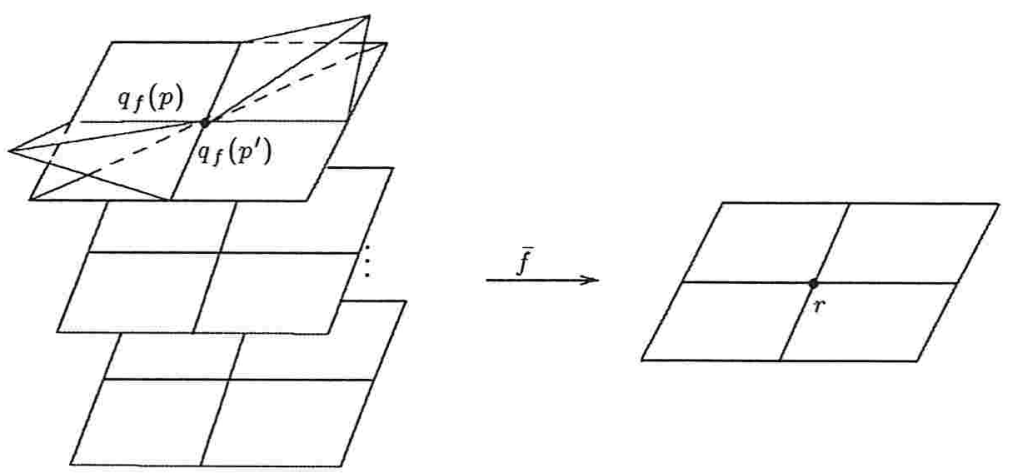

g) $f^{-1}(r) \cap S(f)=f^{-1}(r) \cap S_{1}=\left\{p, p^{\prime}\right\}$ e $q_{f}(p)=q_{f}\left(p^{\prime}\right)$ é um tridente.
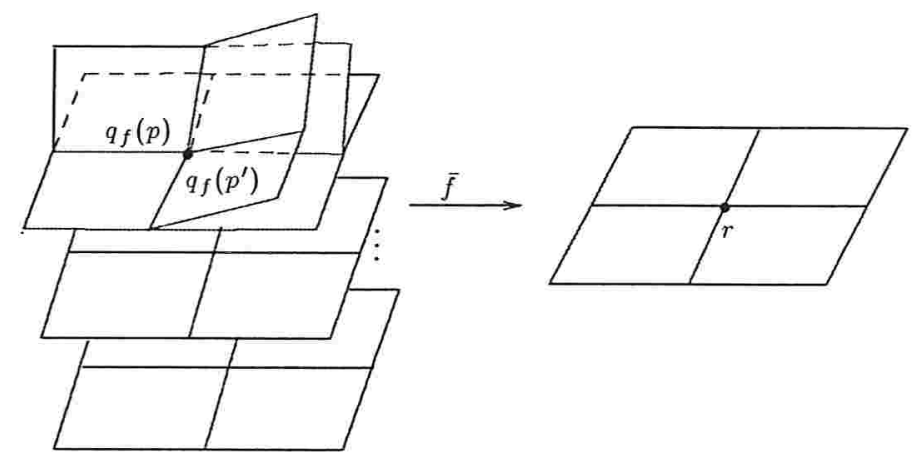

h) $f^{-1}(r) \cap S(f)=f^{-1}(r) \cap C=\{p\}$.

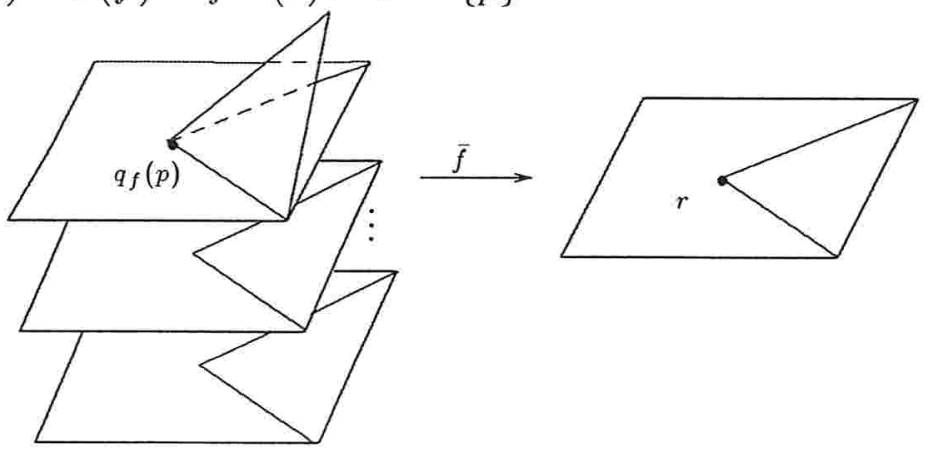


Na próxima seção, faremos uso das representações acima para calcularmos o índice local para os vértices de $M(\bar{f})$.

No decorrer de todo este capítulo utilizamos as notações abaixo. Observamos apenas que, na Seção 5.3 a definição de $I I$ é um pouco diferente.

Notação 5.1.5 Sejam $N^{3}$ uma variedade tridimensional orientável e fechada, $g: N^{3} \rightarrow \mathbb{R}^{2}$ uma aplicação $\mathcal{C}^{\infty}$-estável e $S(g)$ o conjunto singular de g. Fixada $f: K \rightarrow L$ uma triangulação de $g$, sejam $S(f)$ a triangulação de $S(g)$ e $f(S(f))$ a triangulação de $g(S(g))$. Utilizamos as seguintes notações:

- $D=\left\{r \in g(S(g)): g^{-1}(r) \cap S(g)=g^{-1}(r) \cap S_{0}\right.$ consiste de um ponto $\}$,

- $I=\left\{r \in g(S(g)): g^{-1}(r) \cap S(g)=g^{-1}(r) \cap S_{1}\right.$ consiste de um ponto $\}$,

- $D D=\left\{r \in g(S(g)): g^{-1}(r) \cap S(g)=g^{-1}(r) \cap S_{0}\right.$ consiste de dois pontos\},

- $D I=\left\{r \in g(S(g)): g^{-1}(r) \cap S_{0} \neq \emptyset\right.$ e $\left.g^{-1}(r) \cap S_{1} \neq \emptyset\right\}$,

- $I I=\left\{r \in g(S(g)): g^{-1}(r) \cap S(g)=g^{-1}(r) \cap S_{1}\right.$ consiste de dois pontos $p, p^{\prime}$ tais que $\left.q_{g}(p) \neq q_{g}\left(p^{\prime}\right)\right\}$,

- $D C=\left\{r \in g(S(g)): g^{-1}(r) \cap S(g)=g^{-1}(r) \cap S_{1}\right.$ consiste de dois pontos $p, p^{\prime}$ tais que $q_{g}(p)=q_{g}\left(p^{\prime}\right)$ é um cone duplo $\}$,

- $T=\left\{r \in g(S(g)): g^{-1}(r) \cap S(g)=g^{-1}(r) \cap S_{1}\right.$ consiste de dois pontos $p, p^{\prime}$ tais que $q_{g}(p)=q_{g}\left(p^{\prime}\right)$ é um tridente $\}$,

- $C=\left\{r \in g(S(g)): g^{-1}(r) \cap S(g)=g^{-1}(r) \cap C\right\}$ (Note que o segundo $C$ denota o conjunto das cúspides de $g$ contidas em $N^{3}$. Usamos a mesma notação $C$ para estes dois conjuntos diferentes, pois eles correspondem biunívocamente por $g$ ). 
- Usamos as notações $D, I, D D, D I, I I, D C, T$ e $C$ para os subconjuntos correspondentes de $f(S(f))$ também.

- Para denotar o número de elementos de um conjunto finito $X$, usamos $\sharp X$.

Observação 5.1.6 Os números dos vértices contidos em $f(K) \backslash f(S(f)), D$ ou $I$ dependem da escolha da triangulação $f: K \rightarrow L$ de $g$. Mas os números $\sharp D D, \sharp D I, \sharp I I, \sharp D C, \sharp T$ e $\sharp C$ não.

\subsection{O número de singularidades de uma apli- cação $g: N^{3} \rightarrow \mathbb{R}^{2}$}

Sejam $N^{3}$ uma variedade tridimensional orientável e fechada, $g: N^{3} \rightarrow \mathbb{R}^{2}$ uma aplicação $\mathcal{C}^{\infty}$-estável, $f: K \rightarrow L$ uma triangulação de $g$ e $\bar{f}: W_{f} \rightarrow \mathbb{R}^{2}$ a aplicação proveniente da fatorização de Stein de $f$ como na Seção 5.1. No que segue, usamos as notações introduzidas na Seção 5.1 para $g$ e $f$.

Para $k=1,2, \ldots$, considere o conjunto:

$$
M^{k}(\bar{f})=\left\{y \in f(K) \backslash f(S(f)): \sharp\left(\bar{f}^{-1}(y)\right)=k\right\},
$$

onde $\sharp$ significa o número de elementos do conjunto.

Definição 5.2.1 Seja $y \in f(K)$, chamamos $k=\sharp\left(\bar{f}^{-1}(y)\right)$ de multiplicidade de $y$. Analogamente, chamamos $k=\sharp\left(\bar{g}^{-1}(z)\right)$ de multiplicidade de $z$, onde $z \in g\left(N^{3}\right)$.

Observação 5.2.2 As multiplicidades de $y \in f(K)$ e de $z \in g\left(N^{3}\right)$ são números inteiros positivos, pois $\bar{f}$ é uma aplicação simplicial não-degenerada e $\bar{g}^{-1}(z)$ é um conjunto finito de pontos que correspondem às componentes conexas de $g^{-1}(z)$. 
Consideremos a decomposição do conjunto $f(K)$ como sendo a união disjunta:

$$
f(K)=\left(\bigcup_{k \geq 1} M^{k}(\bar{f})\right) \cup f(S(f))
$$

Definição 5.2.3 Sejam $\Delta=f(S(f))$ e $v \in \Delta^{(0)}$, definimos $\operatorname{Ind}_{\bar{f}_{\Delta}}(v)$ como o índice de [BS] aplicado a $\bar{f}_{\Delta}=\left.\bar{f}\right|_{\bar{f}^{-1}(\Delta)}: \bar{f}^{-1}(\Delta) \rightarrow \Delta$.

Observação 5.2.4 De acordo com a caracterização de $\bar{f}$ feita na Observação 5.1.4, em vizinhanças dos vértices $v \in \Delta^{(0)}$, podemos calcular os índices $\operatorname{Ind}_{\bar{f}_{\Delta}}(v)$ como a seguir. Seja $k$ a multiplicidade de cada um dos vértices $v \in \Delta$ abaixo. Da equação (4.2) segue que

$$
\operatorname{Ind}_{\bar{f}_{\Delta}}(v)=\sum_{\tau \ni v} \frac{(-1)^{\operatorname{dim} \tau}}{\operatorname{dim} \tau+1}-\sum_{w \in\left(\bar{f}_{\Delta}^{-1}(v)\right)^{(0)}} \sum_{\sigma \ni w} \frac{(-1)^{\operatorname{dim} \sigma}}{\operatorname{dim} \sigma+1} .
$$

Obtemos assim:

a) $v \in D$.

$$
\operatorname{Ind}_{\bar{f}_{\Delta}}(v)=(1-k)-\frac{1}{2}(2-2 k)=0 .
$$

b) $v \in I$.

$$
\operatorname{Ind}_{\bar{f}_{\Delta}}(v)=(1-k)-\frac{1}{2}(2-2 k)=0 .
$$

c) $v \in D D$.

$$
\operatorname{Ind}_{\bar{f}_{\Delta}}(v)=(1-k)-\frac{1}{2}(4-4 k+2)=1-k+2 k-3=k-2 .
$$

d) $v \in D I$.

$$
\operatorname{Ind}_{\bar{f}_{\Delta}}(v)=(1-k)-\frac{1}{2}(4-4 k)=1-k+2 k-2=k-1 .
$$

e) $v \in I I$. 
$\operatorname{Ind}_{\bar{f}_{\Delta}}(v)=(1-k)-\frac{1}{2}(4-4 k-2)=1-k+2 k-1=k$.

f) $v \in D C$.

$$
\operatorname{Ind}_{\bar{f}_{\Delta}}(v)=(1-k)-\frac{1}{2}(4-4 k)=1-k-2+2 k=k-1 .
$$

g) $v \in T$.

$$
\operatorname{Ind}_{\bar{f}_{\Delta}}(v)=(1-k)-\frac{1}{2}(4-4 k-2)=1-k-1+2 k=k .
$$

h) $v \in C$.

$$
\operatorname{Ind}_{\bar{f}_{\Delta}}(v)=(1-k)-\frac{1}{2}(2-2 k-1)=1-k-\frac{1}{2}+k=\frac{1}{2} .
$$

Obtemos, no próximo teorema, uma relação entre as singularidades de $g$ e certos invariantes topológicos. Para tanto, usamos a seguinte notação:

$D D^{k}$ denota o subconjunto de $D D$ que consiste dos elementos de multiplicidade $k$,

$D I^{k}$ denota o subconjunto de $D I$ que consiste dos elementos de multiplicidade $k$,

$I I^{k}$ denota o subconjunto de $I I$ que consiste dos elementos de multiplicidade $k$,

$D C^{k}$ denota o subconjunto de $D C$ que consiste dos elementos de multiplicidade $k$,

$T^{k}$ denota o subconjunto de $T$ que consiste dos elementos de multiplicidade $k$.

Observação 5.2.5 Observe que: $\sharp D D=\sum_{k \geq 2} \sharp D D^{k}, \sharp D I=\sum_{k \geq 2} \sharp D I^{k}, \sharp I I=$ $\sum_{k \geq 2} \sharp I I^{k}, \sharp D C=\sum_{k \geq 1} \sharp D C^{k}, \sharp T=\sum_{k \geq 1} \sharp T^{k}$. 
Segue, agora, o seguinte:

Teorema 5.2.6 Seja $g: N^{3} \rightarrow \mathbb{R}^{2}$ uma aplicação $\mathcal{C}^{\infty}$-estável, onde $N^{3}$ é uma variedade tridimensional orientável e fechada. Então:

$$
\begin{array}{r}
\chi\left(g\left(N^{3}\right)\right)=\chi\left(W_{g}\right)+\sum_{k \geq 2}(1-k) \chi_{c}\left(M^{k}(\bar{g})\right)+\sum_{k \geq 3}(k-2) \sharp D D^{k}+\sum_{k \geq 2}(k-1) \sharp D I^{k} \\
+\sum_{k \geq 2} k \sharp I I^{k}+\sum_{k \geq 2}(k-1) \sharp D C^{k}+\sum_{k \geq 1} k \sharp T^{k}+\frac{\sharp C}{2},
\end{array}
$$

onde $W_{g}$ é o espaço quociente da fatorização de Stein de $g, \bar{g}$ é a aplicação proveniente da fatorização de Stein de $g, M^{k}(\bar{g})=\left\{y \in g\left(N^{3}\right) \backslash g(S(g))\right.$ : $\left.\sharp\left(\bar{g}^{-1}(y)\right)=k\right\}$ e $\chi_{c}\left(M^{k}(\bar{g})\right)$ é a característica combinatória de $M^{k}(\bar{g})$.

\section{Demonstração:}

Primeiro observe que, pelo Teorema 1.3 .8 sabemos que a fatorização de Stein de $g$ é triangulável. Sejam $f: K \rightarrow L$ uma triangulação de $g$ e $\bar{f}: W_{f} \rightarrow L$ a aplicação não-degenerada da fatorização de Stein de $f$.

Como já vimos, se $\Delta=f(S(f))$, então:

$$
f(K)=\left(\bigcup_{k \geq 1} M^{k}(\bar{f})\right) \cup \Delta .
$$

Também sabemos que $f(K)=\bar{f}\left(W_{f}\right)$ e $W_{f}=\bar{f}^{-1}(f(K))$, então: 


$$
\begin{aligned}
& \chi\left(\bar{f}\left(W_{f}\right)\right)-\chi\left(W_{f}\right)=\chi(f(K))-\chi\left(W_{f}\right) \\
= & \chi\left(\left(\bigcup_{k \geq 1} M^{k}(\bar{f})\right) \cup \Delta\right)-\chi\left(\bar{f}^{-1}\left(\left(\bigcup_{k \geq 1} M^{k}(\bar{f})\right) \cup \Delta\right)\right) \\
= & \chi\left(\left(\bigcup_{k \geq 1} M^{k}(\bar{f})\right) \cup \Delta\right)-\chi\left(\bar{f}^{-1}\left(\bigcup_{k \geq 1} M^{k}(\bar{f})\right) \cup \bar{f}^{-1}(\Delta)\right) \\
= & \chi_{c}\left(\bigcup_{k \geq 1} M^{k}(\bar{f})\right)+\chi(\Delta)-\left(\chi_{c}\left(\bar{f}^{-1}\left(\bigcup_{k \geq 1} M^{k}(\bar{f})\right)\right)+\chi\left(\bar{f}^{-1}(\Delta)\right)\right) \\
= & \chi_{c}\left(\bigcup_{k \geq 1} M^{k}(\bar{f})\right)-\chi_{c}\left(\bar{f}^{-1}\left(\bigcup_{k \geq 1} M^{k}(\bar{f})\right)\right)+\chi(\Delta)-\chi\left(\bar{f}^{-1}(\Delta)\right) \\
= & \sum_{k \geq 1} \chi_{c}\left(M^{k}(\bar{f})\right)-\sum_{k \geq 1} k_{\chi_{c}}\left(M^{k}(\bar{f})\right)+\chi(\Delta)-\chi\left(\bar{f}^{-1}(\Delta)\right) \\
= & \sum_{k \geq 2}(1-k) \chi_{c}\left(M^{k}(\bar{f})\right)+\chi(\Delta)-\chi\left(\bar{f}^{-1}(\Delta)\right) .
\end{aligned}
$$

Do [BS, Theorem 3.3] (veja o Teorema 4.2.4), aplicado a $\bar{f}_{\Delta}=\left.\bar{f}\right|_{\bar{f}^{-1}(\Delta)}$ : $\bar{f}^{-1}(\Delta) \rightarrow \Delta$, obtemos:

$$
\chi(\Delta)-\chi\left(\bar{f}^{-1}(\Delta)\right)=\sum_{v \in \Delta(0)} \operatorname{Ind}_{\bar{f}_{\Delta}}(v)
$$

ou seja,

$$
\chi\left(\bar{f}\left(W_{f}\right)\right)-\chi\left(W_{f}\right)=\sum_{k \geq 2}(1-k) \chi_{c}\left(M^{k}(\bar{f})\right)+\sum_{v \in \Delta^{(0)}} \operatorname{Ind}_{\bar{f}_{\Delta}}(v) .
$$


Utilizando os índices calculados na Observação 5.2.4, temos que:

$$
\begin{aligned}
\chi\left(\bar{f}\left(W_{f}\right)\right)-\chi\left(W_{f}\right) & =\sum_{k \geq 2}(1-k) \chi_{c}\left(M^{k}(\bar{f})\right)+\sum_{v \in D D} \operatorname{Ind}_{\bar{f}_{\Delta}}(v)+\sum_{v \in D I} \operatorname{Ind}_{\bar{f}_{\Delta}}(v) \\
& +\sum_{v \in I I} \operatorname{Ind}_{\bar{f}_{\Delta}}(v)+\sum_{v \in D C} \operatorname{Ind}_{\bar{f}_{\Delta}}(v)+\sum_{v \in T} \operatorname{Ind}_{\bar{f}_{\Delta}}(v) \\
& +\sum_{v \in C} \operatorname{Ind}_{\bar{f}_{\Delta}}(v) \\
& =\sum_{k \geq 2}(1-k) \chi_{c}\left(M^{k}(\bar{f})\right)+\sum_{k \geq 3}(k-2) \sharp D D^{k}+\sum_{k \geq 2}(k-1) \sharp D I^{k} \\
& +\sum_{k \geq 2} k \sharp I I^{k}+\sum_{k \geq 2}(k-1) \sharp D C^{k}+\sum_{k \geq 1} k \sharp T^{k}+\frac{\sharp C}{2} .
\end{aligned}
$$

Portanto, como $f$ é uma triangulação de $g$, segue que:

$$
\begin{aligned}
\chi\left(\bar{g}\left(W_{g}\right)\right)-\chi\left(W_{g}\right) & =\sum_{k \geq 2}(1-k) \chi_{c}\left(M^{k}(\bar{g})\right)+\sum_{k \geq 3}(k-2) \sharp D D^{k}+\sum_{k \geq 2}(k-1) \sharp D I^{k} \\
& +\sum_{k \geq 2} k \sharp I I^{k}+\sum_{k \geq 2}(k-1) \sharp D C^{k}+\sum_{k \geq 1} k \sharp T^{k}+\frac{\sharp C}{2} .
\end{aligned}
$$

Mas, $\bar{g}\left(W_{g}\right)=g\left(N^{3}\right)$; assim,

$$
\begin{aligned}
\chi\left(g\left(N^{3}\right)\right)-\chi\left(W_{g}\right) & =\sum_{k \geq 2}(1-k) \chi_{c}\left(M^{k}(\bar{g})\right)+\sum_{k \geq 3}(k-2) \sharp D D^{k}+\sum_{k \geq 2}(k-1) \sharp D I^{k} \\
& +\sum_{k \geq 2} k \sharp I I^{k}+\sum_{k \geq 2}(k-1) \sharp D C^{k}+\sum_{k \geq 1} k \sharp T^{k}+\frac{\sharp C}{2} .
\end{aligned}
$$

Corolário 5.2.7 Nas condições do Teorema 5.2.6 segue que o número de cúspides é sempre par.

\section{Demonstração:}


Considere a fórmula do Teorema 5.2.6 multiplicada por 2. A fórmula assim obtida $(\bmod 2)$ fica da seguinte forma:

$$
\sharp C \equiv 0(\bmod 2),
$$

ou seja, o número de cúspides de $g$ tem que ser par.

Para melhor compreensão dos exemplos abaixo, segue a seguinte:

Observação 5.2.8 Considere o par $(P, l)$, chamado de $W$-imerso, onde $P$ é um poliedro e $l: P \rightarrow \mathbb{R}^{2}$ uma aplicação simplicial não-degenerada satisfazendo certas condições [MPS, Definition 3.6]. Mata-Lorenzo, em sua tese de doutorado $[\mathrm{ML}]$ mostra que $(P, l)$ sempre pode ser realizado como fatorização de Stein de uma aplicação $\mathcal{C}^{\infty}$-estável $g: N^{3} \rightarrow \mathbb{R}^{2}$, para alguma variedade fechada e orientável $N^{3}$. Um esboço da demonstração do resultado de Mata-Lorenzo [ML] pode ser encontrado em [MPS].

Exemplo 5.2.9 Seja $g: N^{3} \rightarrow \mathbb{R}^{2}$ uma realização do $W$-imerso $\left(W_{g}, \bar{g}\right)$ representado no diagrama abaixo:

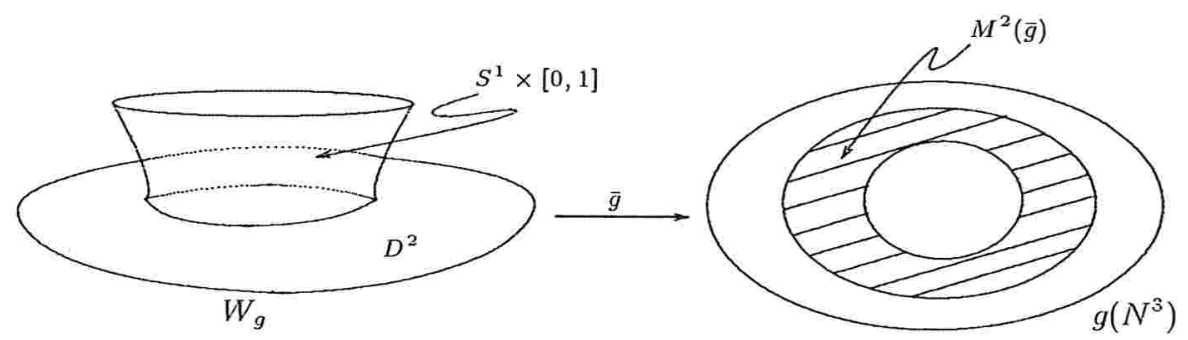

Assim, temos que $\chi\left(g\left(N^{3}\right)\right)=\chi\left(\bar{g}\left(W_{g}\right)\right)=1, \chi\left(W_{g}\right)=1, \chi_{c}\left(M^{2}(\bar{g})\right)=0$, $\sharp D D=\sharp D I=\sharp I I=\sharp D C=\sharp T=\sharp C=0$. Com isso, podemos verificar que a fórmula do Teorema 5.2.6 está satisfeita. 
Exemplo 5.2.10 Seja $g: N^{3} \rightarrow \mathbb{R}^{2}$ uma realização do $W$-imerso $\left(W_{g}, \bar{g}\right)$ representado no diagrama abaixo:
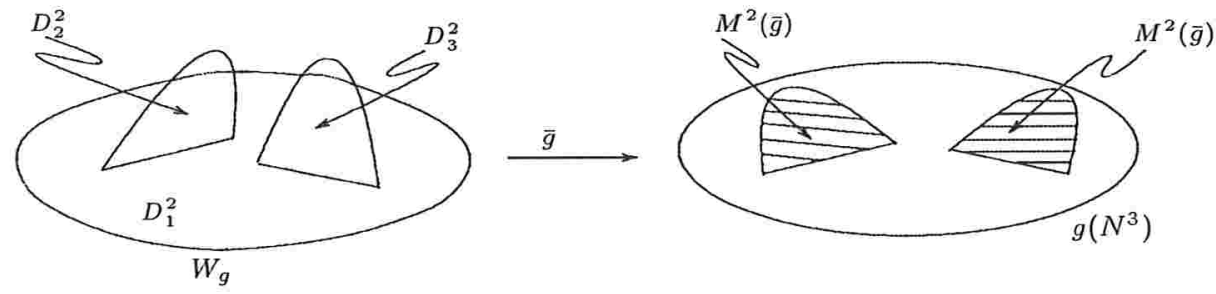

Assim, temos que $\chi\left(g\left(N^{3}\right)\right)=\chi\left(\bar{g}\left(W_{g}\right)\right)=1, \chi\left(W_{g}\right)=1, \chi_{c}\left(M^{2}(\bar{g})\right)=2$, $\sharp D D=\sharp D I=\sharp I I=\sharp D C=\sharp T=0$ e $\sharp C=4$. Com isso, podemos verificar que a fórmula do Teorema 5.2 .6 está satisfeita.

Exemplo 5.2.11 Seja $g: N^{3} \rightarrow \mathbb{R}^{2}$ uma realização do $W$-imerso $\left(W_{g}, \bar{g}\right)$ representado no diagrama abaixo:

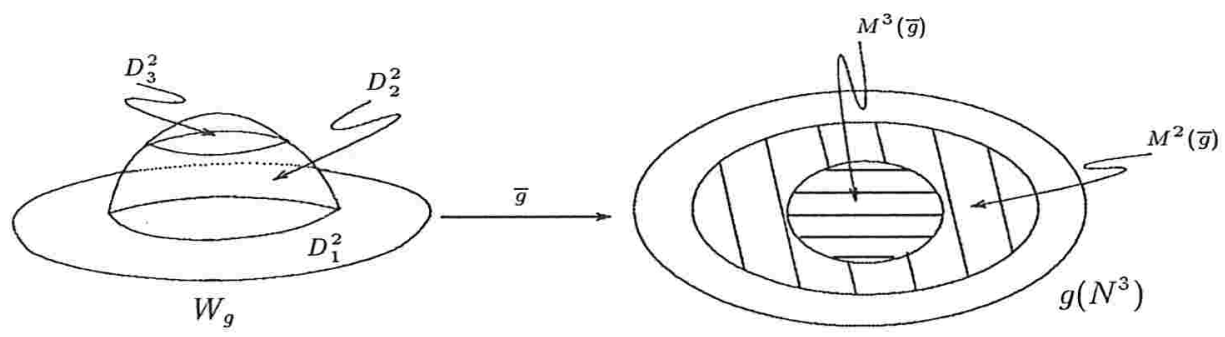

Assim, temos que $\chi\left(g\left(N^{3}\right)\right)=\chi\left(\bar{g}\left(W_{g}\right)\right)=1, \chi\left(W_{g}\right)=3, \chi\left(M^{2}(\bar{g})\right)=0$, $\chi\left(M^{3}(\bar{g})\right)=1$ e $\sharp D D=\sharp D I=\sharp I I=\sharp D C=\sharp T=\sharp C=0$. Com isso, podemos verificar que a fórmula do Teorema 5.2.6 está satisfeita.

Exemplo 5.2.12 Seja $g: N^{3} \rightarrow \mathbb{R}^{2}$ uma realização do $W$-imerso $\left(W_{g}, \bar{g}\right)$ representado no diagrama abaixo: 


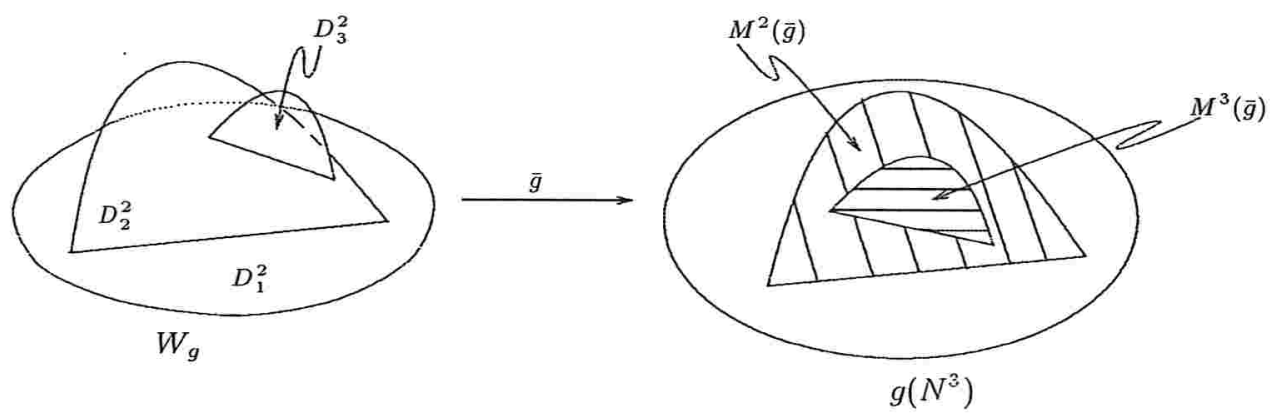

Assim, temos que $\chi\left(g\left(N^{3}\right)\right)=\chi\left(\bar{g}\left(W_{g}\right)\right)=1, \chi\left(W_{g}\right)=1, \chi\left(M^{2}(\bar{g})\right)=0$, $\chi\left(M^{3}(\bar{g})\right)=1$ e $\sharp D D=\sharp D I=\sharp I I=\sharp D C=\sharp T=0$ e $\sharp C=4$. Com isso, podemos verificar que a fórmula do Teorema 5.2.6 está satisfeita.

Exemplo 5.2.13 Seja $g: N^{3} \cdot \rightarrow \mathbb{R}^{2}$ uma realização do $W$-imerso $\left(W_{g}, \bar{g}\right)$ representado no diagrama abaixo:

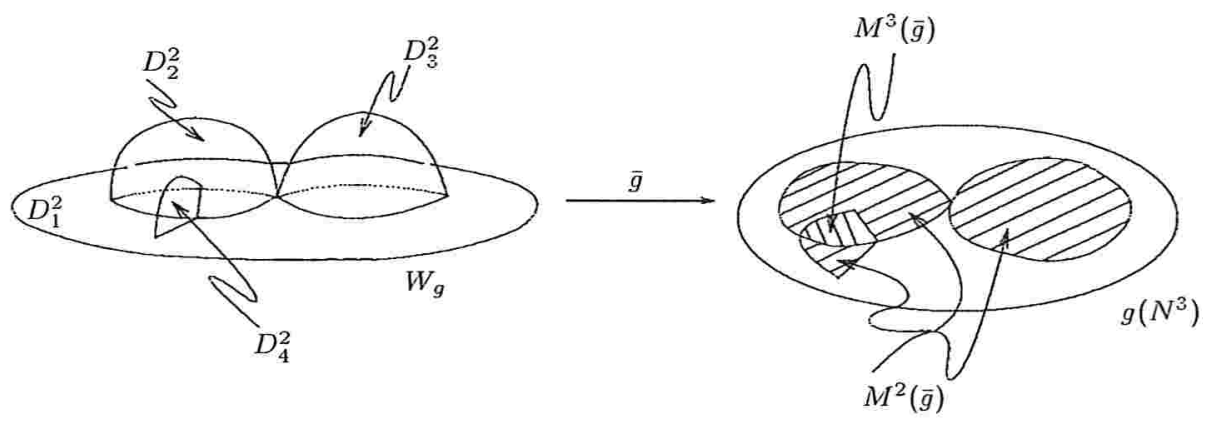

Assim, temos que $\chi\left(g\left(N^{3}\right)\right)=\chi\left(\bar{g}\left(W_{g}\right)\right)=1, \chi\left(W_{g}\right)=3, \chi_{c}\left(M^{2}(\bar{g})\right)=3$, $\chi_{c}\left(M^{3}(\bar{g})\right)=1, \sharp D I^{2}=\sharp D C^{1}=\sharp T^{1}=1$ e $\sharp C=2$. Com isso, podemos verificar que a fórmula do Teorema 5.2.6 está satisfeita.

\section{3 $\mathrm{O}$ índice $\operatorname{Ind}_{f}^{\star}(v)$ e aplicações}

Seja $g: N^{3} \rightarrow \mathbb{R}^{2}$ uma aplicação $\mathcal{C}^{\infty}$-estável, onde $N^{3}$ é uma variedade tridimensional fechada. Objetivando novas relações entre as singularidades 
de $g$ e invariantes topológicos, vamos introduzir agora uma nova definição de índice, que denotaremos por $\operatorname{Ind}_{f}^{\star}(v)$. Apresentamos, nesta seção, uma aplicação simples deste índice para aplicações $\mathcal{C}^{\infty}$-estáveis $N^{3} \rightarrow \mathbb{R}^{2}$; porém, através dele, obtemos resultados bastante interessantes para aplicações $\mathcal{C}^{\infty}$ estáveis $N^{4} \rightarrow \mathbb{R}^{3}$ que serão estudadas no próximo capítulo.

Consideremos uma aplicação simplicial $f: K \rightarrow L$, onde $K$ é um complexo simplicial finito. Primeiramente observemos que $f(K)$ e $K$ podem ser escritos como as seguintes uniões disjuntas:

$$
f(K)=\bigcup_{\tau \in f(K)} \stackrel{\circ}{\tau} \quad \text { e } \quad K=\bigcup_{\tau \in f(K)} f^{-1}(\stackrel{\circ}{\tau}) .
$$

Utilizando as relações anteriores, podemos escrever as características de Euler de $f(K)$ e de $K$ em função das características combinatórias de Euler dos conjuntos $\stackrel{\circ}{\tau}$ e $f^{-1}(\stackrel{\circ}{\tau})$, respectivamente:

$$
\chi(f(K))=\sum_{\tau \in f(K)} \chi_{c}(\stackrel{\circ}{\tau}) \quad \text { e } \quad \chi(K)=\sum_{\tau \in f(K)} \chi_{c}\left(f^{-1}(\stackrel{\circ}{\tau})\right)
$$

Consideremos agora a diferença:

$$
\begin{aligned}
\chi(f(K))-\chi(K) & =\sum_{\tau \in f(K)} \chi_{c}(\stackrel{\circ}{\tau})-\sum_{\tau \in f(K)} \chi_{c}\left(f^{-1}(\stackrel{\circ}{\tau})\right) \\
& =\sum_{\tau \in f(K)}\left(\chi_{c}(\stackrel{\circ}{\tau})-\chi_{c}\left(f^{-1}(\stackrel{\circ}{\tau})\right)\right) .
\end{aligned}
$$

Mas, pela Proposição 2.3.9, temos que:

$$
f^{-1}(\stackrel{\circ}{\tau}) \approx \stackrel{\circ}{\tau} \times f^{-1}\left(b_{\tau}\right)
$$


onde $b_{\tau}$ é o baricentro de $\tau$. Portanto, pela Observação 4.3.2, temos que:

$$
\begin{aligned}
\chi(f(K))-\chi(K) & =\sum_{\tau \in f(K)}\left(\chi_{c}(\stackrel{\circ}{\tau})-\chi_{c}\left(\stackrel{\circ}{\tau} \times f^{-1}\left(b_{\tau}\right)\right)\right) \\
& =\sum_{\tau \in f(K)}\left(\chi_{c}(\stackrel{\circ}{\tau})-\chi_{c}(\stackrel{\circ}{\tau}) \chi\left(f^{-1}\left(b_{\tau}\right)\right)\right) \\
& =\sum_{\tau \in f(K)} \chi_{c}(\stackrel{\circ}{\tau})\left(1-\chi\left(f^{-1}\left(b_{\tau}\right)\right)\right) .
\end{aligned}
$$

Lembrando ainda que $\chi_{c}(\stackrel{\circ}{\tau})=(-1)^{\operatorname{dim} \tau}$, segue que:

$$
\chi(f(K))-\chi(K)=\sum_{v \in f(K)^{(0)}} \sum_{\tau \ni v} \frac{(-1)^{\operatorname{dim} \tau}}{1+\operatorname{dim} \tau}\left(1-\chi\left(f^{-1}\left(b_{\tau}\right)\right)\right) .
$$

Isto sugere a seguinte:

Definição 5.3.1 $\operatorname{Ind}_{f}^{\star}(v)=\sum_{\tau \ni v} \frac{(-1)^{\operatorname{dim} \tau}}{1+\operatorname{dim} \tau}\left(1-\chi\left(f^{-1}\left(b_{\tau}\right)\right)\right)$.

Assim, segue naturalmente que:

$$
\chi(f(K))-\chi(K)=\sum_{v \in f(K)^{(0)}} \operatorname{Ind}_{f}^{\star}(v) .
$$

Nesta seção vamos utilizar a seguinte:

Notação 5.3.2 Sejam $N^{3}$ uma variedade tridimensional fechada, $g: N^{3} \rightarrow$ $\mathbb{R}^{2}$ uma aplicação $\mathcal{C}^{\infty}$-estável e $S(g)$ o conjunto singular de $g$. Fixada $f$ : $K \rightarrow L$ uma triangulação de $g$, sejam $S(f)$ a triangulação de $S(g)$ e $f(S(f))$ a triangulação de $g(S(g))$. Considere:

- $D=\left\{r \in g(S(g)): g^{-1}(r) \cap S(g)=g^{-1}(r) \cap S_{0}\right.$ consiste de um ponto $\}$,

- $I=\left\{r \in g(S(g)): g^{-1}(r) \cap S(g)=g^{-1}(r) \cap S_{1}\right.$ consiste de um ponto $\}$, 
- $D D=\left\{r \in g(S(g)): g^{-1}(r) \cap S(g)=g^{-1}(r) \cap S_{0}\right.$ consiste de dois pontos\},

- $D I=\left\{r \in g(S(g)): g^{-1}(r) \cap S_{0} \neq \emptyset\right.$ e $\left.g^{-1}(r) \cap S_{1} \neq \emptyset\right\}$,

- $I I=\left\{r \in g(S(g)): g^{-1}(r) \cap S(g)=g^{-1}(r) \cap S_{1}\right.$ consiste de dois pontos\},

- $C=\left\{r \in g(S(g)): g^{-1}(r) \cap S(g)=g^{-1}(r) \cap C\right\}$ (Note que o segundo $C$ denota o conjunto das cúspides de $g$ contidas em $N^{3}$. Usamos a mesma notação $C$ para estes dois conjuntos diferentes, pois eles correspondem biunívocamente por $g$ ).

- Usamos as notações $D, I, D D, D I, I I$ e $C$ para os subconjuntos correspondentes de $f(S(f))$ também.

- Para denotar o número de elementos de um conjunto finito $X$, usamos $\sharp X$.

Observação 5.3.3 Note que a notação acima é diferente da anterior, pois aqui $I I$ é a união de $I I, D C$ e $T$ da notação das Seções 5.1 e 5.2 , mas isto vale somente quando $N^{3}$ é orientável. Note que estamos trabalhando com a variedade $N^{3}$ que pode não ser orientável.

No que segue, utilizando (5.4) obtemos uma nova fórmula que nos permite demonstrar de maneira mais simples, sem utilizar a dualidade de Poincaré, o seguinte resultado: "A característica de Euler de toda variedade diferenciável fechada de dimensão três é nula".

Vamos, agora, calcular os índices $\operatorname{Ind}_{f}^{\star}(v)$. Para tanto considere $f: K \rightarrow$ $L$ uma triangulação de uma aplicação $\mathcal{C}^{\infty}$-estável $g: N^{3} \rightarrow \mathbb{R}^{2}$, onde $N^{3}$ é uma variedade fechada, que pode ser não orientável (veja [Ve1],[Ve2]). 
Para $v \in(f(K))^{(0)}$, introduzimos as seguintes notações:

$a_{v}^{0} \quad$ o número de arestas de $f(K)$ que contém $v$ e cujos interiores estão contidos em $f(K) \backslash f(S(f))$,

$b_{v}^{0} \quad$ o número de faces de $f(K)$ que contém $v$ e cujos interiores estão contidos em $f(K) \backslash f(S(f))$,

$$
I_{v}^{0}=-\frac{a_{v}^{0}}{2}+\frac{b_{v}^{0}}{3}
$$

Observação 5.3.4 Apresentamos abaixo os cálculos dos índices $\operatorname{Ind}_{f}^{\star}(v)$ para $v \in(f(K))^{(0)}$, relativos à aplicação $f$ que é uma triangulação de $g$. Levamos em consideração que todas as componentes regulares das fibras de $g$ são subvariedades unidimensionais de $N^{3}$ que são homeomorfas a $S^{1}$ [Mi, pg.55]. Portanto, as características de Euler das componentes regulares das fibras são iguais a zero. Para os cálculos dos índices abaixo levamos em consideração as figuras do Apêndice B, onde estão enumerados as características de Euler das fibras $f^{-1}(v)$ nos seguintes casos:

a) $v \in(f(K))^{(0)} \backslash f(S(f))$.

$$
\begin{aligned}
\operatorname{Ind}_{f}^{\star}(v) & =(1-0)-\frac{a_{v}^{0}}{2}(1-0)+\frac{b_{v}^{0}}{3}(1-0) \\
& =1-\frac{a_{v}^{0}}{2}+\frac{b_{v}^{0}}{3} \\
& =1+I_{v}^{0} .
\end{aligned}
$$

b) $v \in(f(K))^{(0)} \cap D$.

$$
\begin{aligned}
\operatorname{Ind}_{f}^{*}(v) & =(1-1)-\frac{a_{v}^{0}}{2}(1-0)-\frac{2}{2}(1-1)+\frac{b_{v}^{0}}{3}(1-0) \\
& =-\frac{a_{v}^{0}}{2}+\frac{b_{v}^{0}}{3} \\
& =I_{v}^{0} .
\end{aligned}
$$


c) $v \in(f(K))^{(0)} \cap I$.

$$
\begin{aligned}
\operatorname{Ind}_{f}^{\star}(v) & =(1+1)-\frac{a_{v}^{0}}{2}(1-0)-\frac{2}{2}(1+1)+\frac{b_{v}^{0}}{3}(1-0) \\
& =-\frac{a_{v}^{0}}{2}+\frac{b_{v}^{0}}{3} \\
& =I_{v}^{0} .
\end{aligned}
$$

d) $v \in D D$.

$$
\begin{aligned}
\operatorname{Ind}_{f}^{\star}(v) & =(1-2)-\frac{a_{v}^{0}}{2}(1-0)-\frac{4}{2}(1-1)+\frac{b_{v}^{0}}{3}(1-0) \\
& =-\frac{a_{v}^{0}}{2}+\frac{b_{v}^{0}}{3}-1 \\
& =I_{v}^{0}-1 .
\end{aligned}
$$

e) $v \in D I$.

$$
\begin{aligned}
\operatorname{Ind}_{f}^{\star}(v) & =(1-0)-\frac{a_{v}^{0}}{2}(1-0)-\frac{2}{2}(1-1)-\frac{2}{2}(1+1)+\frac{b_{v}^{0}}{3}(1-0) \\
& =-\frac{a_{v}^{0}}{2}+\frac{b_{v}^{0}}{3}-1 \\
& =I_{v}^{0}-1 .
\end{aligned}
$$

f) $v \in I I$.

$$
\begin{aligned}
\operatorname{Ind}_{f}^{*}(v) & =(1+2)-\frac{a_{v}^{0}}{2}(1-0)-\frac{4}{2}(1+1)+\frac{b_{v}^{0}}{3}(1-0) \\
& =-\frac{a_{v}^{0}}{2}+\frac{b_{v}^{0}}{3}-1 \\
& =I_{v}^{0}-1 .
\end{aligned}
$$

g) $v \in C$.

$$
\begin{aligned}
\operatorname{Ind}_{f}^{*}(v) & =(1-0)-\frac{a_{v}^{0}}{2}(1-0)-\frac{1}{2}(1-1)-\frac{1}{2}(1+1)+\frac{b_{v}^{0}}{3}(1-0) \\
& =-\frac{a_{v}^{0}}{2}+\frac{b_{v}^{0}}{3} \\
& =I_{v}^{0}
\end{aligned}
$$


Teorema 5.3.5 Seja $h: X \rightarrow Y$ uma imersão diferenciável entre duas variedades diferenciáveis. Então $h$ é $\mathcal{C}^{\infty}$-estável se, e somente se, $h$ tem somente cruzamentos normais [GG, Chapter III, Definition 3.1].

Demonstração: [GG, pg.85].

Lema 5.3.6 Seja $h: X \rightarrow \mathbb{R}^{2}$ uma imersão com cruzamentos normais, onde $X$ é uma união finita de esferas unidimensionais $S^{1}$. Então a característica de Euler de $h(X)$ é igual ao oposto do número de cruzamentos da imagem de $h$.

\section{Demonstração:}

Primeiramente decomponha $h(X)$ como sendo a união disjunta dos conjuntos $U_{1}=\left\{p \in h(X): \sharp h^{-1}(p)=1\right\}$ e $U_{2}=\left\{p \in h(X): \sharp h^{-1}(p)=2\right\}$.

Como $h$ é uma aplicação $\mathcal{C}^{\infty}$-estável, segundo Verona [Ve1], [Ve2], $h$ é triangulável. Sejam $l: A \rightarrow B$ uma triangulação de $h$ e $L_{i}$ o subconjunto de $B$ que corresponde a $U_{i}, i=1,2$.

Os índices $\operatorname{Ind}_{l}(v)$, de $[\mathrm{BS}]$, dos vértices de $l(A)$ são dados por:

a) se $v \in L_{1}^{(0)}$, então $\operatorname{Ind}_{l}(v)=1(1-1)-\frac{1}{2}(2-2)=0$,

b) se $v \in L_{2}$, então $\operatorname{Ind}_{l}(v)=1(1-2)-\frac{1}{2}(2-2)=-1$.

Por [BS] sabemos que $\chi(l(A))-\chi(A)=\sum_{v \in(l(A))^{(0)}} \operatorname{Ind}_{l}(v)$, ou seja,

$$
\begin{aligned}
\chi(l(A))-\chi(A) & =\sum_{v \in L_{1}^{(0)}} \operatorname{Ind}_{l}(v)+\sum_{v \in L_{2}} \operatorname{Ind}_{l}(v) \\
& =\sum_{v \in L_{1}^{(0)}} 0+\sum_{v \in L_{2}}(-1) \\
& =-\sharp L_{2},
\end{aligned}
$$


onde $\sharp L_{2}$ é o número de elementos de $L_{2}$, que é exatamente o número de cruzamentos da imagem de $h$. Como $\chi(l(A))=\chi(h(X))$ e $\chi(A)=0$, segue que $\chi(h(X))=-\sharp L_{2}$.

Corolário 5.3.7 Seja $g: N^{3} \rightarrow \mathbb{R}^{2}$ uma aplicação $\mathcal{C}^{\infty}$-estável, onde $N^{3}$ é uma variedade tridimensional e fechada. Então $\chi(g(S(g)))=-\sharp D D-\sharp D I-$ $\sharp I I$.

\section{Demonstração:}

Como $g$ é $\mathcal{C}^{\infty}$-estável, pela Proposição 5.1.1, temos que $\left.g\right|_{S(g) \backslash C}$ é uma imersão com cruzamentos normais. Note que $\left.g\right|_{S(g)}$ pode não ser uma imersão com cruzamentos normais, mas é fácil mostrar que $\left.g\right|_{S(g)}$ é topologicamente equivalente a uma imersão com cruzamentos normais. Pelo Lema 5.3.6 temos que $\chi(g(S(g)))$ é o oposto do número de cruzamentos da imagem de $\left.g\right|_{S(g)}$ e este, por sua vez, é igual a $\sharp D D+\sharp D I+\sharp I I$. Portanto, temos $\chi(g(S(g)))=$ $-\sharp D D-\sharp D I-\sharp I I$.

Teorema 5.3.8 Seja $N^{3}$ uma variedade diferenciável tridimensional e fechada. Então $\chi\left(N^{3}\right)=0$.

\section{Demonstração:}

Toda variedade fechada tridimensional $N^{3}$ admite uma aplicação $\mathcal{C}^{\infty}$ estável $g: N^{3} \rightarrow \mathbb{R}^{2}$. Se $f: K \rightarrow L$ é uma triangulação de $g$, então valem os índices $\operatorname{Ind}_{f}^{\star}(v)$ calculados na Observação 5.3.4, e temos: 


$$
\begin{aligned}
& \chi(f(K))-\chi(K)=\sum_{v \in f(K)^{(0)}} \operatorname{Ind}_{f}^{\star}(v) \\
& =\sum_{v \in(f(K))^{(0)} \backslash f(S(f))} \operatorname{Ind}_{f}^{\star}(v)+\sum_{v \in(f(K))^{(0)} \cap D} \operatorname{Ind}_{f}^{\star}(v) \\
& +\sum_{v \in(f(K))^{(0)} \cap I} \operatorname{Ind}_{f}^{\star}(v)+\sum_{v \in D D} \operatorname{Ind}_{f}^{\star}(v)+\sum_{v \in D I} \operatorname{Ind}_{f}^{\star}(v) \\
& +\quad \sum_{v \in I I} \operatorname{Ind}_{f}^{\star}(v)+\sum_{v \in C} \operatorname{Ind}_{f}^{\star}(v) \\
& =\sum_{v \in(f(K))^{(0)} \backslash f(S(f))}\left(1+I_{v}^{0}\right)+\sum_{v \in(f(K))^{(0)} \cap D} I_{v}^{0}+\sum_{v \in(f(K))^{(0)} \cap I} I_{v}^{0} \\
& +\quad \sum_{v \in D D}\left(I_{v}^{0}-1\right)+\sum_{v \in D I}\left(I_{v}^{0}-1\right)+\sum_{v \in I I}\left(I_{v}^{0}-1\right) \\
& +\quad \sum_{v \in C} I_{v}^{0} \\
& =\sum_{v \in(f(K))^{(0)} \backslash f(S(f))}\left(1+I_{v}^{0}\right)+\sum_{v \in(f(S(f)))^{(0)}} I_{v}^{0}-\sharp D D-\sharp D I-\sharp I I(5.8) \\
& =\chi_{c}(f(K) \backslash f(S(f)))-\sharp D D-\sharp D I-\sharp I I .
\end{aligned}
$$

As igualdades acima valem:

(5.5) pela fórmula (5.4);

(5.6) pois $f(K)^{(0)}$ é a união disjunta dos conjuntos $(f(K))^{(0)} \backslash f(S(f))$, $(f(K))^{(0)} \cap D,(f(K))^{(0)} \cap I, D D, D I, I I$ e $C$;

(5.7) pela Observação 5.3.4; 
(5.8) pois $(f(S(f)))^{(0)}$ é a união disjunta dos conjuntos $(f(K))^{(0)} \cap D,(f(K))^{(0)} \cap$ $I, D D, D I, I I$ e $C, \sum_{v \in D D} 1=\sharp D D, \sum_{v \in D I} 1=\sharp D I, \sum_{v \in I I} 1=\sharp I I ;$

(5.9) pois $\sum_{v \in(f(K))^{(0)} \backslash f(S(f))}\left(1+I_{v}^{0}\right)+\sum_{v \in f(S(f))^{(0)}} I_{v}^{0}=\chi_{c}(f(K) \backslash f(S(f)))$.

Pelo Corolário 5.3.7 segue que $\chi(f(S(f)))=-\sharp D D-\sharp D I-\sharp I I$, ou seja,

$$
\chi(f(K))-\chi(K)=\chi_{c}(f(K) \backslash f(S(f)))+\chi(f(S(f))) .
$$

Mas,

$$
\chi(f(K))=\chi_{c}(f(K) \backslash f(S(f)))+\chi(f(S(f))),
$$

então $\chi(K)=0$, ou seja, $\chi\left(N^{3}\right)=0$. 


\section{Capítulo 6}

\section{Fórmulas referentes a aplicações estáveis de uma 4-variedade no $\mathbb{R}^{3}$}

$\mathrm{Na}$ linha de estudo desenvolvido no Capítulo 5, analisamos a seguir, aplicações $g: N^{4} \rightarrow \mathbb{R}^{3}$, onde $g$ é uma aplicação $\mathcal{C}^{\infty}$-estável e $N^{4}$ é uma variedade 4-dimensional fechada e orientável. Analogamente, obtemos duas fórmulas relacionando as singularidades da aplicação com invariantes topológicos. Ambas são fórmulas relacionando grandezas em $\mathbb{Z}$; a partir delas obtemos outra (mod 2) que possibilita tirar conclusões sobre a paridade das singularidades de g. Para isso, adotamos um procedimento análogo ao do capítulo anterior.

\subsection{Estudo local de aplicações $N^{4} \rightarrow \mathbb{R}^{3}$ estáveis}

Iniciamos com o já bem conhecido resultado:

Proposição 6.1.1 Seja $N^{4}$ uma variedade 4-dimensional e fechada. Uma aplicação diferenciável $g: N^{4} \rightarrow \mathbb{R}^{3}$ é $\mathcal{C}^{\infty}$-estável se, e somente se, $g$ satisfaz as seguintes condições locais e globais: para todo $p$ no conjunto singular de $g, S(g)$, existem coordenadas locais $(x, y, z, w)$ em torno de $p$ e coordenadas 
locais $(X, Y, Z)$ em torno de $g(p)$ tais que $g$ tem uma das seguintes formas:

L1) $\left\{\begin{array}{l}X \circ g=x, \\ Y \circ g=y, \\ Z \circ g=z^{2}+w^{2} ;\end{array} \quad(p:\right.$ ponto de dobra definida $)$

L2) $\left\{\begin{array}{l}X \circ g=x, \\ Y \circ g=y, \\ Z \circ g=z^{2}-w^{2} ;\end{array} \quad(p:\right.$ ponto de dobra indefinida $)$

L3) $\left\{\begin{array}{l}X \circ g=x, \\ Y \circ g=y, \\ Z \circ g=z^{3}+x z-w^{2} ;\end{array} \quad(p:\right.$ ponto de cúspide $)$

L4) $\left\{\begin{array}{l}X \circ g=x, \\ Y \circ g=y, \\ Z \circ g=z^{4}+x z^{2}+y z+w^{2} ;\end{array} \quad(p:\right.$ rabo de andorinha definido $)$

L5) $\left\{\begin{array}{l}X \circ g=x, \\ Y \circ g=y, \\ Z \circ g=z^{4}+x z^{2}+y z-w^{2} ;\end{array} \quad(p:\right.$ rabo de andorinha indefinido $)$

e os "multi-germs" de $\left.g\right|_{S(g)}$ são equivalentes a uma das seguintes figuras:
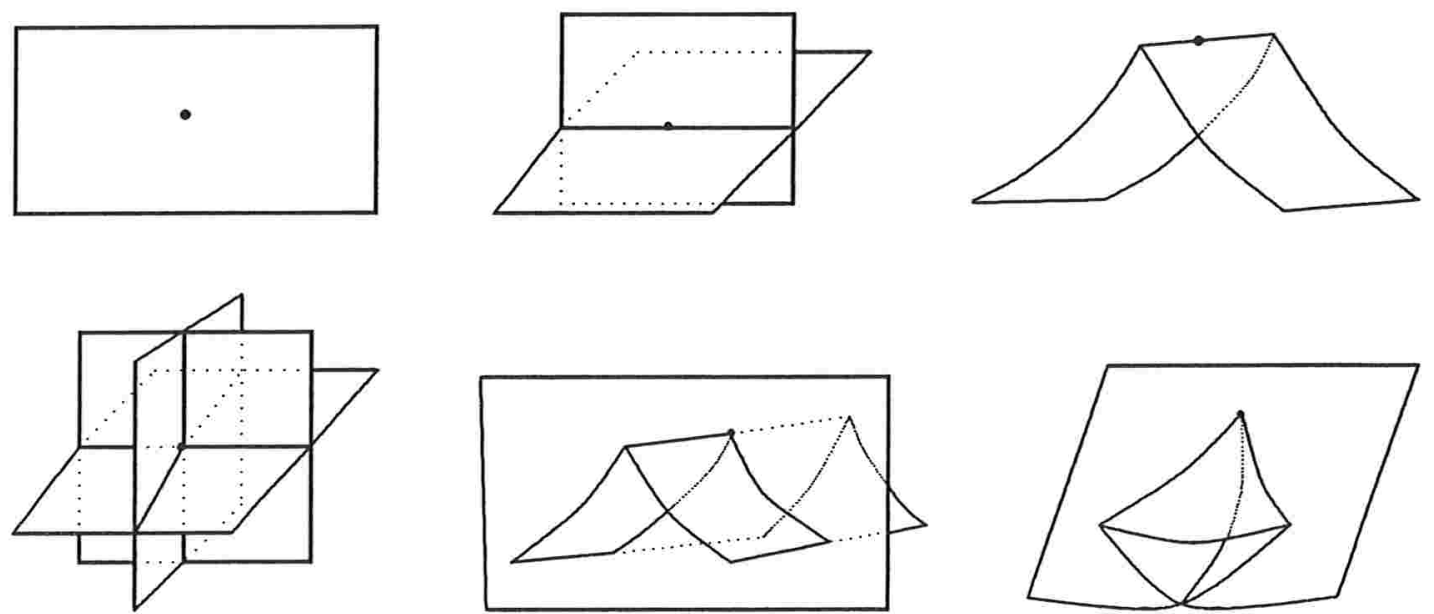
Considere os "multi-germs" descritos na Proposição 6.1.1. Dependendo das superfícies consideradas, ou seja, se forem imagens do conjunto de dobra definida (def) ou de dobra indefinida (indef), os "multi-germs" podem se dividir em vários casos como podemos ver a seguir. As notações estão estabelecidas logo após as figuras.

Observação 6.1.2 Os "multi-germs" de $\left.g\right|_{S(g)}$ se dividem nos seguintes tipos:

a) Imagem de uma superfície de dobra (2 tipos).
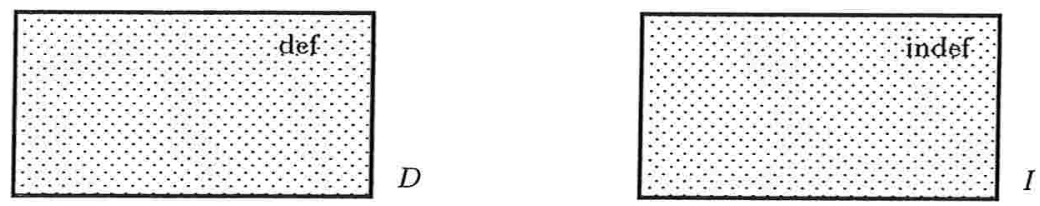

b) Imagem de uma curva de cúspide (1 tipo).

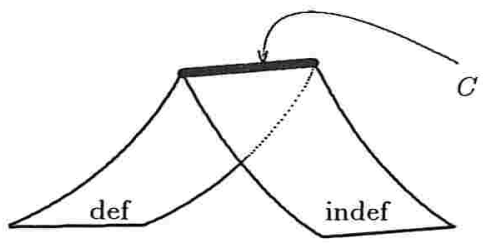

c) Imagem de um ponto de rabo de andorinha (2 tipos).
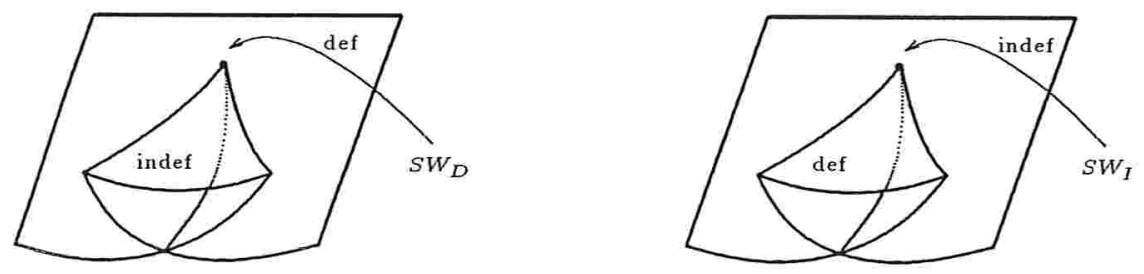
Quando $N^{4}$ for orientável temos as seguintes igualdades: $S W_{D}=S W_{1}$ e $S W_{I}=S W_{2} \cup S W_{3}$.

d) Imagem de duas superfícies de dobra transversais (3 tipos).
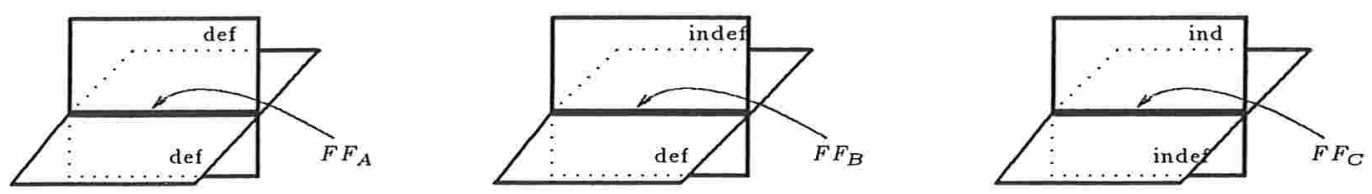

Quando $N^{4}$ for orientável temos as seguintes igualdades: $F F_{A}=F F_{1}$, $F F_{B}=F F_{2}$ e $F F_{C}=F F_{3} \cup F F_{4} \cup F F_{5}$.

e) Imagem de três superfícies de dobra transversais (4 tipos).
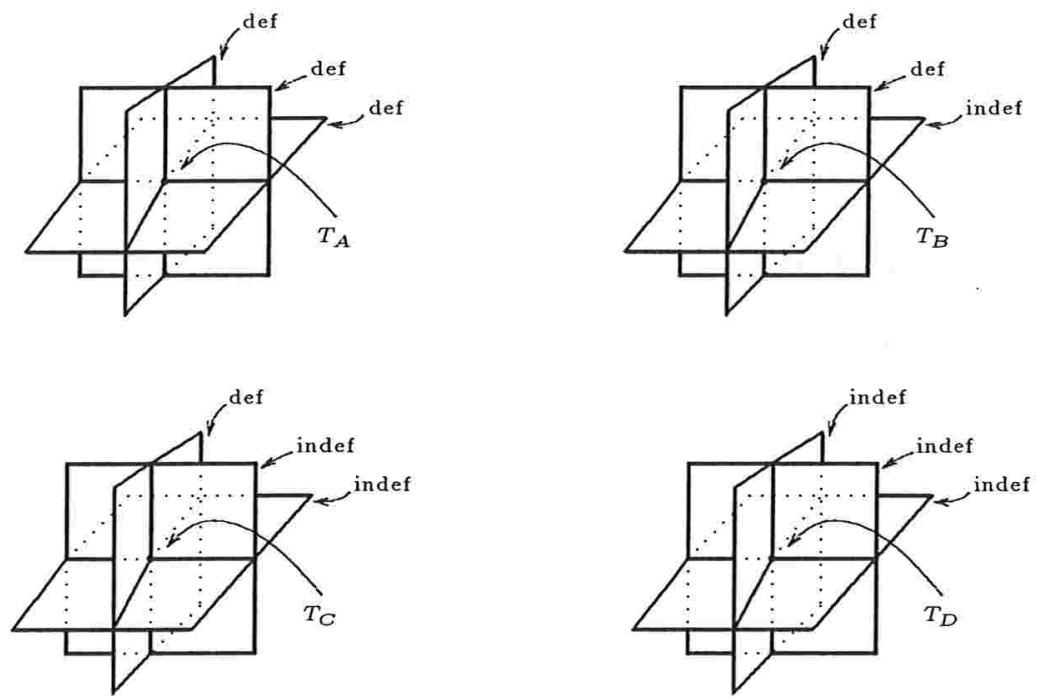

Quando $N^{4}$ for orientável temos as seguintes igualdades: $T_{A}=T_{1}, T_{B}=$ $T_{2}, T_{C}=T_{3} \cup T_{5} \cup T_{6}$ e $T_{D}=T_{4} \cup T_{7} \cup T_{8} \cup \cdots \cup T_{13}$. 
f) Imagem de uma curva de cúspide com uma superfície de dobra (2 tipos).
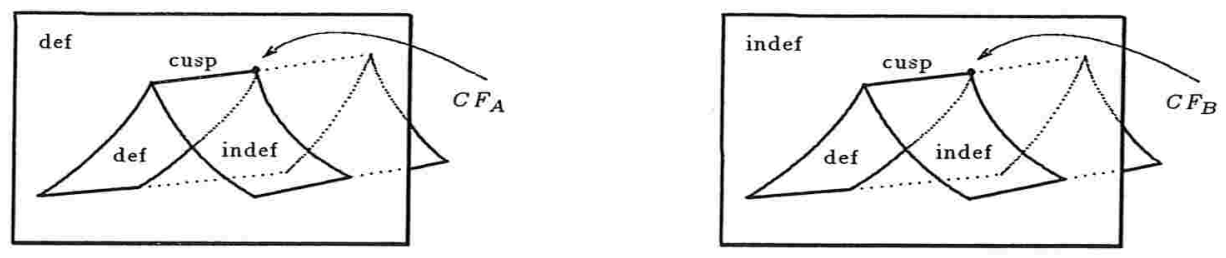

Quando $N^{4}$ for orientável temos as seguintes igualdades: $C F_{A}=C F_{2}$ e $C F_{B}=C F_{1} \cup C F_{3}$.

Conforme a forma das fibras de $g$ em torno de cada valor, os germes semi-locais de $g$ podem se dividir em mais casos além dos citados acima; no caso orientável, eles estão relacionados no Apêndice C. Uma classificação, no caso $N^{3} \rightarrow \mathbb{R}^{2}$, está relacionada no Apêndice A e pode ser encontrada, por exemplo, em [KLP], [Le]; e no caso $N^{4} \rightarrow \mathbb{R}^{2}$ em [Fr], [PF].

\subsection{Uma fórmula sobre o número de singula- ridades}

Nesta seção, repetimos o processo utilizado na Seção 5.2 para aplicações estáveis $g: N^{4} \rightarrow \mathbb{R}^{3}$, obtendo uma fórmula em $\mathbb{Z}$ e outra $(\bmod 2)$ que nos auxilia no estudo qualitativo e quantitativo das singularidades de $g$.

Iniciamos esta seção com a seguinte:

Notação 6.2.1 Sejam $N^{4}$ uma variedade 4-dimensional orientável e fechada, $g: N^{4} \rightarrow \mathbb{R}^{3}$ uma aplicação $\mathcal{C}^{\infty}$-estável e $S(g)$ o conjunto singular de $g$. Fixada $f: K \rightarrow L$ uma triangulação de $g$ ([Ve1], [Ve2]), sejam $S(f)$ a 
triangulação de $S(g)$ e $f(S(f))$ a triangulação de $g(S(g))$. Sejam, ainda, $S_{0} \subset N^{4}$ o conjunto dos pontos de dobras definidas, $S_{1} \subset N^{4}$ o conjunto dos pontos dobras indefinidas, $C \subset N^{4}$ o conjunto dos pontos de cúspides, $W_{0} \subset N^{4}$ o conjunto dos pontos de rabos de andorinha definidos, e $W_{1} \subset N^{4}$ o conjunto dos pontos de rabos de andorinha indefinidos. Usamos as mesmas notações para os subconjuntos de $K$ correspondentes. Então, definimos:

- $D=\left\{r \in g(S(g)): g^{-1}(r) \cap S(g)=g^{-1}(r) \cap S_{0}\right.$ consiste de um ponto $\}$,

- $I=\left\{r \in g(S(g)): g^{-1}(r) \cap S(g)=g^{-1}(r) \cap S_{1}\right.$ consiste de um ponto $\}$,

- $C=\left\{r \in g(S(g)): g^{-1}(r) \cap S(g)=g^{-1}(r) \cap C\right\}$ (Note que o segundo $C$ denota o conjunto das cúspides de $g$ contidas em $N^{4}$. Usamos a mesma notaçã்o $C$ para estes dois conjuntos diferentes, pois eles correspondem biunívocamente por $g$ ),

- $F F_{i}=\left\{r \in g(S(g)): g^{-1}(r) \cap S(g)\right.$ consiste de dois pontos de dobra e a fibra $g^{-1}(r)$ é do tipo $i$ de acordo com o Apêndice $\left.C\right\}, i=1,2,3,4,5$,

- $F F=\bigcup_{i=1}^{5} F F_{i}$

- $T_{i}=\left\{r \in g(S(g)): g^{-1}(r) \cap S(g)\right.$ consiste de três pontos de dobra e a fibra $g^{-1}(r)$ é do tipo $i$ de acordo com o Apêndice $\left.C\right\}, i=1,2, \ldots, 13$,

- $T=\bigcup_{i=1}^{13} T_{i}$

- $C F_{i}=\left\{r \in g(S(g)): g^{-1}(r) \cap S(g)\right.$ consiste de um ponto de dobra e uma cúspide, e a fibra $g^{-1}(r)$ é do tipo $i$ de acordo com o Apêndice C\}, $i=1,2,3$,

- $C F=\bigcup_{i=1}^{3} C F_{i}$ 
- $S W_{i}=\left\{r \in g(S(g)): g^{-1}(r) \cap S(g)\right.$ consiste de um ponto de rabo de andorinha e a fibra $g^{-1}(r)$ é do tipo $i$ de acordo com o Apêndice C\}, $i=1,2,3$,

- $S W=\bigcup_{i=1}^{3} S W_{i}$.

- Usamos as notações $D, I, C, F F_{i}, F F, T, T, C F_{i}, C F, S W_{i}$ e $S W$ para os subconjuntos correspondentes de $f(S(f))$ também.

- Para denotar o número de elementos de um conjunto finito $X$, usamos $\sharp X$.

Observação 6.2.2 Os números dos vértices contidos em $f(K) \backslash f(S(f)), D$, $I, C$ ou $F F_{i}$ dependem da escolha da triangulação $f: K \rightarrow L$ de $g$. Mas os números $\sharp T_{i}, \sharp C F_{i}$ e $\sharp S W_{i}$ não.

Considere a fatorização de Stein de $f$ (veja o Teorema 3.2.15 e a Observação 3.2 .18$)$ :

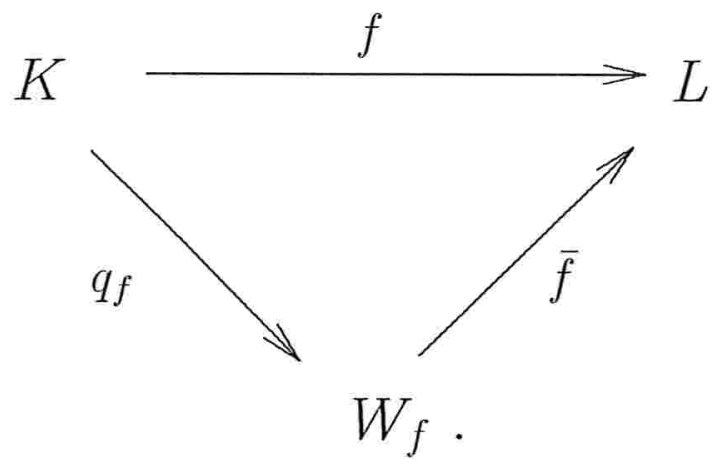

Definição 6.2.3 Seja $\Delta=C \cup F F \cup T \cup C F \cup S W$ e $v \in \Delta^{(0)}$, então $\operatorname{Ind}_{\bar{f}_{\Delta}}(v)$ é o índice de [BS], relativo à aplicação $\bar{f}_{\Delta}=\bar{f}_{\bar{f}^{-1}(\Delta)}: \bar{f}^{-1}(\Delta) \rightarrow \Delta$.

Observação 6.2.4 Calculamos abaixo os índices $\operatorname{Ind}_{\bar{f}_{\Delta}}(v)$ das imagens dos pontos singulares definidos acima, onde $k$ é a multiplicidade de cada vértice $v$ (Definição 5.2.1). Para melhor compreensão dos cálculos veja Apêndice C. 
I) Pontos triplos:

a) $v \in T_{1}$.

$$
\operatorname{Ind}_{\bar{f}_{\Delta}}(v)=(1-k)-\frac{1}{2}(3-3(k-1)+3-3 k)=2 k-\frac{7}{2} .
$$

b) $v \in T_{2}$.

$$
\operatorname{Ind}_{\bar{f}_{\Delta}}(v)=(1-k)-\frac{1}{2}(2-2(k-1)+3-3 k+1-1(k+1))=2 k-\frac{5}{2} .
$$

c) $v \in T_{3}$.

$$
\operatorname{Ind}_{\bar{f}_{\Delta}}(v)=(1-k)-\frac{1}{2}(1-1(k-1)+3-3 k+2-2(k+1))=2 k-\frac{3}{2} .
$$

d) $v \in T_{4}$.

$$
\operatorname{Ind}_{\bar{f}_{\Delta}}(v)=(1-k)-\frac{1}{2}(3-3 k+3-3(k+1))=2 k-\frac{1}{2} .
$$

e) $v \in T_{5}$.

$$
\operatorname{Ind}_{\bar{f}_{\Delta}}(v)=(1-k)-\frac{1}{2}(1-1(k-1)+3-3 k+2-2(k+1))=2 k-\frac{3}{2} .
$$

f) $v \in T_{6}$.

$$
\operatorname{Ind}_{\bar{f}_{\Delta}}(v)=(1-k)-\frac{1}{2}(1-1(k-1)+5-5 k)=2 k-\frac{5}{2} .
$$

g) $v \in T_{7}$.

$$
\operatorname{Ind}_{\bar{f}_{\Delta}}(v)=(1-k)-\frac{1}{2}(3-3 k+3-3(k+1))=2 k-\frac{1}{2} .
$$

h) $v \in T_{8}$.

$$
\operatorname{Ind}_{f_{\Delta}}(v)=(1-k)-\frac{1}{2}(5-5 k+1-1(k+1))=2 k-\frac{3}{2} .
$$

i) $v \in T_{9}$.

$$
\operatorname{Ind}_{\bar{f}_{\Delta}}(v)=(1-k)-\frac{1}{2}(3-3 k+3-3(k+1))=2 k-\frac{1}{2} .
$$


j) $v \in T_{10}$.

$$
\operatorname{Ind}_{\bar{f}_{\Delta}}(v)=(1-k)-\frac{1}{2}(3-3 k+3-3(k+1))=2 k-\frac{1}{2} .
$$

l) $v \in T_{11}$.

$$
\operatorname{Ind}_{\bar{f}_{\Delta}}(v)=(1-k)-\frac{1}{2}(5-5 k+1-1(k+1))=2 k-\frac{3}{2} .
$$

m) $v \in T_{12}$.

$$
\operatorname{Ind}_{\bar{f}_{\Delta}}(v)=(1-k)-\frac{1}{2}(6-6 k)=2 k-\frac{4}{2} .
$$

n) $v \in T_{13}$.

$$
\operatorname{Ind}_{\bar{f}_{\Delta}}(v)=(1-k)-\frac{1}{2}(6-6 k)=2 k-\frac{4}{2} .
$$

II) Pontos duplos (dobra + cúspide):

a) $v \in C F_{1}$.

$$
\operatorname{Ind}_{\bar{f}_{\Delta}}(v)=(1-k)-\frac{1}{2}(2-2 k+2-2(k+1))=k .
$$

b) $v \in C F_{2}$.

$$
\operatorname{Ind}_{\bar{f}_{\Delta}}(v)=(1-k)-\frac{1}{2}(1-1(k-1)+2-2 k+1-1(k+1))=k-1 .
$$

c) $v \in C F_{3}$.

$$
\operatorname{Ind}_{\bar{f}_{\Delta}}(v)=(1-k)-\frac{1}{2}(2-2 k+2-2(k+1))=k .
$$

III) Rabo de andorinha:

a) $v \in S W_{1}$.

$$
\operatorname{Ind}_{\bar{f}_{\Delta}}(v)=(1-k)-\frac{1}{2}(2-2 k+1-1(k+1))=\frac{k}{2} .
$$

b) $v \in S W_{2}$. 


$$
\operatorname{Ind}_{\bar{f}_{\Delta}}(v)=(1-k)-\frac{1}{2}(1-k+2-2(k+1))=\frac{k}{2}+\frac{1}{2} \text {. }
$$

c) $v \in S W_{3}$.

$$
\operatorname{Ind}_{\bar{f}_{\Delta}}(v)=(1-k)-\frac{1}{2}(3-3 k)=\frac{k}{2}-\frac{1}{2} .
$$

Observação 6.2.5 Os índices para os demais pontos são iguais a zero.

Para enunciarmos o próximo teorema necessitamos das seguintes notações:

$\sharp T_{i}^{k}$ é o número de elementos de $T_{i}, i=1, \ldots, 13$, de multiplicidade $k$, $\sharp C F_{i}^{k}$ é o número de elementos de $C F_{i}, i=1,2,3$, de multiplicidade $k$, $\sharp S W_{i}^{k}$ é o número de elementos de $S W_{i}, i=1,2,3$, de multiplicidade $k$.

Segue o seguinte:

Teorema 6.2.6 Seja $g: N^{4} \rightarrow \mathbb{R}^{3}$ uma aplicação $\mathcal{C}^{\infty}$-estável, onde $N^{4}$ é uma variedade 4-dimensional orientável e fechada. Então:

$$
\begin{aligned}
& \chi\left(g\left(N^{4}\right)\right)-\chi\left(W_{g}\right)=\sum_{k \geq 2}(1-k) \chi_{c}\left(M_{3}^{k}(\bar{g})\right)+\sum_{k \geq 2}(1-k) \chi_{c}\left(M_{2}^{k}(\bar{g})\right) \\
& +\sum_{k \geq 3}\left(2 k-\frac{7}{2}\right) \sharp T_{1}^{k}+\sum_{k \geq 3}\left(2 k-\frac{5}{2}\right) \sharp T_{2}^{k}+\sum_{k \geq 3}\left(2 k-\frac{3}{2}\right) \sharp T_{3}^{k}+\sum_{k \geq 3}\left(2 k-\frac{1}{2}\right) \sharp T_{4}^{k} \\
& +\sum_{k \geq 3}\left(2 k-\frac{3}{2}\right) \sharp T_{5}^{k}+\sum_{k \geq 3}\left(2 k-\frac{5}{2}\right) \sharp T_{6}^{k}+\sum_{k \geq 3}\left(2 k-\frac{1}{2}\right) \sharp T_{7}^{k}+\sum_{k \geq 3}\left(2 k-\frac{3}{2}\right) \sharp T_{8}^{k} \\
& +\sum_{k \geq 3}\left(2 k-\frac{1}{2}\right) \sharp T_{9}^{k}+\sum_{k \geq 3}\left(2 k-\frac{1}{2}\right) \sharp T_{10}^{k}+\sum_{k \geq 3}\left(2 k-\frac{3}{2}\right) \sharp T_{11}^{k}+\sum_{k \geq 3}\left(2 k-\frac{4}{2}\right) \sharp T_{12}^{k} \\
& \quad+\sum_{k \geq 3}\left(2 k-\frac{4}{2}\right) \sharp T_{13}^{k}+\sum_{k \geq 2} k \sharp C F_{1}^{k}+\sum_{k \geq 2}(k-1) \sharp C F_{2}^{k}+\sum_{k \geq 2} k \sharp C F_{3}^{k}
\end{aligned}
$$




$$
+\sum_{k \geq 1} \frac{k}{2} \sharp S W_{1}^{k}+\sum_{k \geq 1}\left(\frac{k}{2}+\frac{1}{2}\right) \sharp S W_{2}^{k}+\sum_{k \geq 1}\left(\frac{k}{2}-\frac{1}{2}\right) \sharp S W_{3}^{k},
$$

onde $M_{3}^{k}(\bar{g})=\left\{y \in g(N) \backslash g(S(g)): \sharp\left(\bar{g}^{-1}(y)\right)=k\right\}, \chi_{c}\left(M_{3}^{k}(\bar{g})\right)$ é a característica combinatória de $M_{3}^{k}(\bar{g}), \Delta=C \cup F F \cup T \cup C F \cup S W, M_{2}^{k}(\bar{g})=\{y \in$ $\left.g(S(g)) \backslash \Delta: \sharp\left(\bar{g}^{-1}(y)\right)=k\right\}$ e $\chi_{c}\left(M_{2}^{k}(\bar{g})\right)$ é a característica combinatória de $M_{2}^{k}(\bar{g})$.

\section{Demonstração:}

Seguimos os mesmos passos da demonstração do Teorema 5.2.6. Sendo $f: K \rightarrow L$ uma triangulação de $g$ e $\bar{f}$ uma triangulação de $\bar{g}$ proveniente da fatorização de Stein de $g$, consideramos a seguinte união disjunta:

$$
f(K)=\left(\bigcup_{k=1}^{n}\left(M_{3}^{k}(\bar{f}) \cup M_{2}^{k}(\bar{f})\right)\right) \cup \Delta,
$$

onde $n$ é a maior multiplicidade que um ponto de $f(K)$ pode assumir e $M_{i}^{k}(\bar{f})$ é o subconjunto de $f(K)$ que corresponde a $M_{i}^{k}(\bar{g})$ para $i=2,3$.

Como $f(K)=\bar{f}\left(W_{f}\right)$ e $W_{f}=\bar{f}^{-1}(f(K))$, utilizando os índices calculados na Observação 6.2.4, temos:

$$
\begin{aligned}
& \chi\left(\bar{f}\left(W_{f}\right)\right)-\chi\left(W_{f}\right)=\chi(f(K))-\chi\left(W_{f}\right) \\
& \quad=\chi\left(\left(\bigcup_{k \geq 1}\left(M_{3}^{k}(\bar{f}) \cup M_{2}^{k}(\bar{f})\right)\right) \cup \Delta\right) \\
& \quad-\chi\left(\bar{f}^{-1}\left(\left(\bigcup_{k \geq 1}\left(M_{3}^{k}(\bar{f}) \cup M_{2}^{k}(\bar{f})\right)\right) \cup \Delta\right)\right) \\
& \quad=\chi_{c}\left(\bigcup_{k \geq 1} M_{3}^{k}(\bar{f})\right)+\chi_{c}\left(\bigcup_{k \geq 1} M_{2}^{k}(\bar{f})\right)+\chi(\Delta)
\end{aligned}
$$




$$
\begin{aligned}
& -\chi_{c}\left(\bar{f}^{-1}\left(\bigcup_{k \geq 1} M_{3}^{k}(\bar{f})\right)\right)-\chi_{c}\left(\bar{f}^{-1}\left(\bigcup_{k \geq 1} M_{2}^{k}(\bar{f})\right)\right)-\chi\left(\bar{f}^{-1}(\Delta)\right) \\
& =\chi_{c}\left(\bigcup_{k \geq 1} M_{3}^{k}(\bar{f})\right)-\chi_{c}\left(\bar{f}^{-1}\left(\bigcup_{k \geq 1} M_{3}^{k}(\bar{f})\right)\right)+\chi_{c}\left(\bigcup_{k \geq 1} M_{2}^{k}(\bar{f})\right) \\
& -\chi_{c}\left(\bar{f}^{-1}\left(\bigcup_{k \geq 1} M_{2}^{k}(\bar{f})\right)\right)+\chi(\Delta)-\chi\left(\bar{f}^{-1}(\Delta)\right) \\
& =\sum_{k \geq 2}(1-k) \chi_{c}\left(M_{3}^{k}(\bar{f})\right)+\sum_{k \geq 2}(1-k) \chi_{c}\left(M_{2}^{k}(\bar{f})\right)+\chi(\Delta)-\chi\left(\bar{f}^{-1}(\Delta)\right) \\
& =\sum_{k \geq 2}(1-k) \chi_{c}\left(M_{3}^{k}(\bar{f})\right)+\sum_{k \geq 2}(1-k) \chi_{c}\left(M_{2}^{k}(\bar{f})\right)+\sum_{v \in \Delta^{(0)}} \operatorname{Ind}_{\bar{f}_{\Delta}}(v) \\
& =\sum_{k \geq 2}(1-k) \chi_{c}\left(M_{3}^{k}(\bar{f})\right)+\sum_{k \geq 2}(1-k) \chi_{c}\left(M_{2}^{k}(\bar{f})\right)+\sum_{v \in T_{1}} \operatorname{Ind}_{\bar{f}_{\Delta}}(v) \\
& +\sum_{v \in T_{2}} \operatorname{Ind}_{\bar{f}_{\Delta}}(v)+\sum_{v \in T_{3}} \operatorname{Ind}_{\bar{f}_{\Delta}}(v)+\sum_{v \in T_{4}} \operatorname{Ind}_{\bar{f}_{\Delta}}(v) \\
& +\sum_{v \in T_{5}} \operatorname{Ind}_{\bar{f}_{\Delta}}(v)+\sum_{v \in T_{6}} \operatorname{Ind}_{\bar{f}_{\Delta}}(v)+\sum_{v \in T_{7}} \operatorname{Ind}_{\bar{f}_{\Delta}}(v) \\
& +\sum_{v \in T_{8}} \operatorname{Ind}_{\bar{f}_{\Delta}}(v)+\sum_{v \in T_{9}} \operatorname{Ind}_{\bar{f}_{\Delta}}(v)+\sum_{v \in T_{10}} \operatorname{Ind}_{\bar{f}_{\Delta}}(v) \\
& +\sum_{v \in T_{11}} \operatorname{Ind}_{\bar{f}_{\Delta}}(v)+\sum_{v \in T_{12}} \operatorname{Ind}_{\bar{f}_{\Delta}}(v)+\sum_{v \in T_{13}} \operatorname{Ind}_{\bar{f}_{\Delta}}(v) \\
& +\sum_{v \in C F_{1}} \operatorname{Ind}_{\bar{f}_{\Delta}}(v)+\sum_{v \in C F_{2}} \operatorname{Ind}_{\bar{f}_{\Delta}}(v)+\sum_{v \in C F_{3}} \operatorname{Ind}_{\bar{f}_{\Delta}}(v) \\
& +\sum_{v \in S W_{1}} \operatorname{Ind}_{\bar{f}_{\Delta}}(v)+\sum_{v \in S W_{2}} \operatorname{Ind}_{\bar{f}_{\Delta}}(v)+\sum_{v \in S W_{3}} \operatorname{Ind}_{\bar{f}_{\Delta}}(v)
\end{aligned}
$$




$$
\begin{aligned}
& =\sum_{k \geq 2}(1-k) \chi_{c}\left(M_{3}^{k}(\bar{f})\right)+\sum_{k \geq 2}(1-k) \chi_{c}\left(M_{2}^{k}(\bar{f})\right)+\sum_{k \geq 3}\left(2 k-\frac{7}{2}\right) \sharp T_{1}^{k} \\
& +\sum_{k \geq 3}\left(2 k-\frac{5}{2}\right) \sharp T_{2}^{k}+\sum_{k \geq 3}\left(2 k-\frac{3}{2}\right) \sharp T_{3}^{k}+\sum_{k \geq 3}\left(2 k-\frac{1}{2}\right) \sharp T_{4}^{k}+\sum_{k \geq 3}\left(2 k-\frac{3}{2}\right) \sharp T_{5}^{k} \\
& +\sum_{k \geq 3}\left(2 k-\frac{5}{2}\right) \sharp T_{6}^{k}+\sum_{k \geq 3}\left(2 k-\frac{1}{2}\right) \sharp T_{7}^{k}+\sum_{k \geq 3}\left(2 k-\frac{3}{2}\right) \sharp T_{8}^{k}+\sum_{k \geq 3}\left(2 k-\frac{1}{2}\right) \sharp T_{9}^{k} \\
& +\sum_{k \geq 3}\left(2 k-\frac{1}{2}\right) \sharp T_{10}^{k}+\sum_{k \geq 3}\left(2 k-\frac{3}{2}\right) \sharp T_{11}^{k}+\sum_{k \geq 3}\left(2 k-\frac{4}{2}\right) \sharp T_{12}^{k} \\
& +\sum_{k \geq 3}\left(2 k-\frac{4}{2}\right) \sharp T_{13}^{k}+\sum_{k \geq 2} k \sharp C F_{1}^{k}+\sum_{k \geq 2}(k-1) \sharp C F_{2}^{k}+\sum_{k \geq 2} k \sharp C F_{3}^{k} \\
& +\sum_{k \geq 1} \frac{k}{2} \sharp S W_{1}^{k}+\sum_{k \geq 1}\left(\frac{k}{2}+\frac{1}{2}\right) \sharp S W_{2}^{k}+\sum_{k \geq 1}\left(\frac{k}{2}-\frac{1}{2}\right) \sharp S W_{3}^{k} .
\end{aligned}
$$

Como $f$ é uma triangulação de $g$, segue que:

$$
\begin{aligned}
& \chi\left(g\left(N^{4}\right)\right)-\chi\left(W_{g}\right)=\sum_{k \geq 2}(1-k) \chi_{c}\left(M_{3}^{k}(\bar{g})\right)+\sum_{k \geq 2}(1-k) \chi_{c}\left(M_{2}^{k}(\bar{g})\right) \\
& +\sum_{k \geq 3}\left(2 k-\frac{7}{2}\right) \sharp T_{1}^{k}+\sum_{k \geq 3}\left(2 k-\frac{5}{2}\right) \sharp T_{2}^{k}+\sum_{k \geq 3}\left(2 k-\frac{3}{2}\right) \sharp T_{3}^{k}+\sum_{k \geq 3}\left(2 k-\frac{1}{2}\right) \sharp T_{4}^{k} \\
& +\sum_{k \geq 3}\left(2 k-\frac{3}{2}\right) \sharp T_{5}^{k}+\sum_{k \geq 3}\left(2 k-\frac{5}{2}\right) \sharp T_{6}^{k}+\sum_{k \geq 3}\left(2 k-\frac{1}{2}\right) \sharp T_{7}^{k}+\sum_{k \geq 3}\left(2 k-\frac{3}{2}\right) \sharp T_{8}^{k} \\
& +\sum_{k \geq 3}\left(2 k-\frac{1}{2}\right) \sharp T_{9}^{k}+\sum_{k \geq 3}\left(2 k-\frac{1}{2}\right) \sharp T_{10}^{k}+\sum_{k \geq 3}\left(2 k-\frac{3}{2}\right) \sharp T_{11}^{k}+\sum_{k \geq 3}\left(2 k-\frac{4}{2}\right) \sharp T_{12}^{k} \\
& +\sum_{k \geq 3}\left(2 k-\frac{4}{2}\right) \sharp T_{13}^{k}+\sum_{k \geq 2} k \sharp C F_{1}^{k}+\sum_{k \geq 2}(k-1) \sharp C F_{2}^{k}+\sum_{k \geq 2} k \sharp C F_{3}^{k} \\
& \quad+\sum_{k \geq 1} \frac{k}{2} \sharp S W_{1}^{k}+\sum_{k \geq 1}\left(\frac{k}{2}+\frac{1}{2}\right) \sharp S W_{2}^{k}+\sum_{k \geq 1}\left(\frac{k}{2}-\frac{1}{2}\right) \sharp S W_{3}^{k} .
\end{aligned}
$$


Corolário 6.2.7 Nas mesmas hipóteses do Teorema 6.2 .6 segue que:

$$
\sharp T-\left(\sharp T_{12}+\sharp T_{13}\right)+\sum_{k: \text { impar }} \sharp S W_{1}^{k}+\sum_{k \text { :par }}\left(\sharp S W_{2}^{k}+\sharp S W_{3}^{k}\right) \equiv 0(\bmod 2) .
$$

\section{Demonstração:}

Do Teorema 6.2.6 temos:

$$
\begin{aligned}
& \chi\left(g\left(N^{4}\right)\right)-\chi\left(W_{g}\right)=\sum_{k \geq 2}(1-k) \chi_{c}\left(M_{3}^{k}(\bar{g})\right)+\sum_{k \geq 2}(1-k) \chi_{c}\left(M_{2}^{k}(\bar{g})\right) \\
& +\sum_{k \geq 3}\left(2 k-\frac{7}{2}\right) \sharp T_{1}^{k}+\sum_{k \geq 3}\left(2 k-\frac{5}{2}\right) \sharp T_{2}^{k}+\sum_{k \geq 3}\left(2 k-\frac{3}{2}\right) \sharp T_{3}^{k}+\sum_{k \geq 3}\left(2 k-\frac{1}{2}\right) \sharp T_{4}^{k} \\
& +\sum_{k \geq 3}\left(2 k-\frac{3}{2}\right) \sharp T_{5}^{k}+\sum_{k \geq 3}\left(2 k-\frac{5}{2}\right) \sharp T_{6}^{k}+\sum_{k \geq 3}\left(2 k-\frac{1}{2}\right) \sharp T_{7}^{k}+\sum_{k \geq 3}\left(2 k-\frac{3}{2}\right) \sharp T_{8}^{k} \\
& +\sum_{k \geq 3}\left(2 k-\frac{1}{2}\right) \sharp T_{9}^{k}+\sum_{k \geq 3}\left(2 k-\frac{1}{2}\right) \sharp T_{10}^{k}+\sum_{k \geq 3}\left(2 k-\frac{3}{2}\right) \sharp T_{11}^{k}+\sum_{k \geq 3}\left(2 k-\frac{4}{2}\right) \sharp T_{12}^{k} \\
& +\sum_{k \geq 3}\left(2 k-\frac{4}{2}\right) \sharp T_{13}^{k}+\sum_{k \geq 2} k \sharp C F_{1}^{k}+\sum_{k \geq 2}(k-1) \sharp C F_{2}^{k}+\sum_{k \geq 2} k \sharp C F_{3}^{k} \\
& \quad+\sum_{k \geq 1} \frac{k}{2} \sharp S W_{1}^{k}+\sum_{k \geq 1}\left(\frac{k}{2}+\frac{1}{2}\right) \sharp S W_{2}^{k}+\sum_{k \geq 1}\left(\frac{k}{2}-\frac{1}{2}\right) \sharp S W_{3}^{k} .
\end{aligned}
$$

Multiplicando a equação (6.2) por 2, segue que:

$$
2 \chi\left(g\left(N^{4}\right)\right)-2 \chi\left(W_{g}\right)=2 \sum_{k \geq 2}(1-k) \chi_{c}\left(M_{3}^{k}(\bar{g})\right)+2 \sum_{k \geq 2}(1-k) \chi_{c}\left(M_{2}^{k}(\bar{g})\right)
$$




$$
\begin{aligned}
& +\sum_{k \geq 3}(4 k-7) \sharp T_{1}^{k}+\sum_{k \geq 3}(4 k-5) \sharp T_{2}^{k}+\sum_{k \geq 3}(4 k-3) \sharp T_{3}^{k}+\sum_{k \geq 3}(4 k-1) \sharp T_{4}^{k} \\
& +\sum_{k \geq 3}(4 k-3) \sharp T_{5}^{k}+\sum_{k \geq 3}(4 k-5) \sharp T_{6}^{k}+\sum_{k \geq 3}(4 k-1) \sharp T_{7}^{k}+\sum_{k \geq 3}(4 k-3) \sharp T_{8}^{k} \\
& +\sum_{k \geq 3}(4 k-1) \sharp T_{9}^{k}+\sum_{k \geq 3}(4 k-1) \sharp T_{10}^{k}+\sum_{k \geq 3}(4 k-3) \sharp T_{11}^{k}+\sum_{k \geq 3}(4 k-4) \sharp T_{12}^{k} \\
& +\sum_{k \geq 3}(4 k-4) \sharp T_{13}^{k}+\sum_{k \geq 2} 2 k \sharp C F_{1}^{k}+\sum_{k \geq 2} 2(k-1) \sharp C F_{2}^{k}+\sum_{k \geq 2} 2 k \sharp C F_{3}^{k} \\
& +\sum_{k \geq 1} k \sharp S W_{1}^{k}+\sum_{k \geq 1}(k+1) \sharp S W_{2}^{k}+\sum_{k \geq 1}(k-1) \sharp S W_{3}^{k} .
\end{aligned}
$$

Considerando a equação $(6.3)(\bmod 2)$, temos:

$$
\sum_{i=1}^{11} \sharp T_{i}+\sum_{k: \text { impar }} \sharp S W_{1}^{k}+\sum_{k: \text { par }}\left(\sharp S W_{2}^{k}+\sharp S W_{3}^{k}\right) \equiv 0(\bmod 2) \text {. }
$$

Mas, $\sharp T=\sum_{i=1}^{13} \sharp T_{i}$, portanto, da equação (6.4), segue que:

$$
\sharp T-\left(\sharp T_{12}+\sharp T_{13}\right)+\sum_{k: \text { ímpar }} \sharp S W_{1}^{k}+\sum_{k \text { :par }}\left(\sharp S W_{2}^{k}+\sharp S W_{3}^{k}\right) \equiv 0(\bmod 2) .
$$

Exemplo 6.2.8 Considere uma aplicação $\mathcal{C}^{\infty}$-estável $f: \mathbb{C P} P^{2} \sharp 2 \overline{\mathbb{C} P^{2}} \rightarrow \mathbb{R}^{3}$ cujas singularidades são apenas dobras. O conjunto $f(S(f))$ consiste de 3 esferas que são imagens de superfícies de dobra definida e uma "Boy surface" formada pela imagem de uma superfície de dobra indefinida. Ou seja, $f$ tem somente um ponto triplo, não tem nenhum rabo de andorinha e nem cúspide. 
Neste caso, temos que $\sharp S W_{1}^{k}=\sharp S W_{2}^{k}=\sharp S W_{3}^{k}=0$, para $\forall k \in \mathbb{Z}$; e pelo Corolário 6.2.7, segue que:

$$
\sharp T-\left(\sharp T_{12}+\sharp T_{13}\right) \equiv 0(\bmod 2),
$$

ou seja, o ponto triplo é do tipo $T_{12}$ ou $T_{13}$, pois $\sharp T=1$.

Exemplo 6.2.9 No [Ko, Theorem 2.1, pg.6], Kobayashi apresenta uma aplicação estável $f_{1}: \mathbb{C} P^{2} \rightarrow \mathbb{R}^{3}$ com as seguinte propriedades:

1) O conjunto dos pontos singulares $S\left(f_{1}\right)$ é difeomorfo a $\mathbb{R} P^{2}$;

2) A aplicação $f_{1}$ tem 6 rabos de andorinha, 6 arcos de cúspides e algumas regiões de dobras;

3) O conjunto dos valores singulares $f_{1}\left(S\left(f_{1}\right)\right) \subset \mathbb{R}^{3}$ é a superfície singular ilustrada na Figura 6.1.

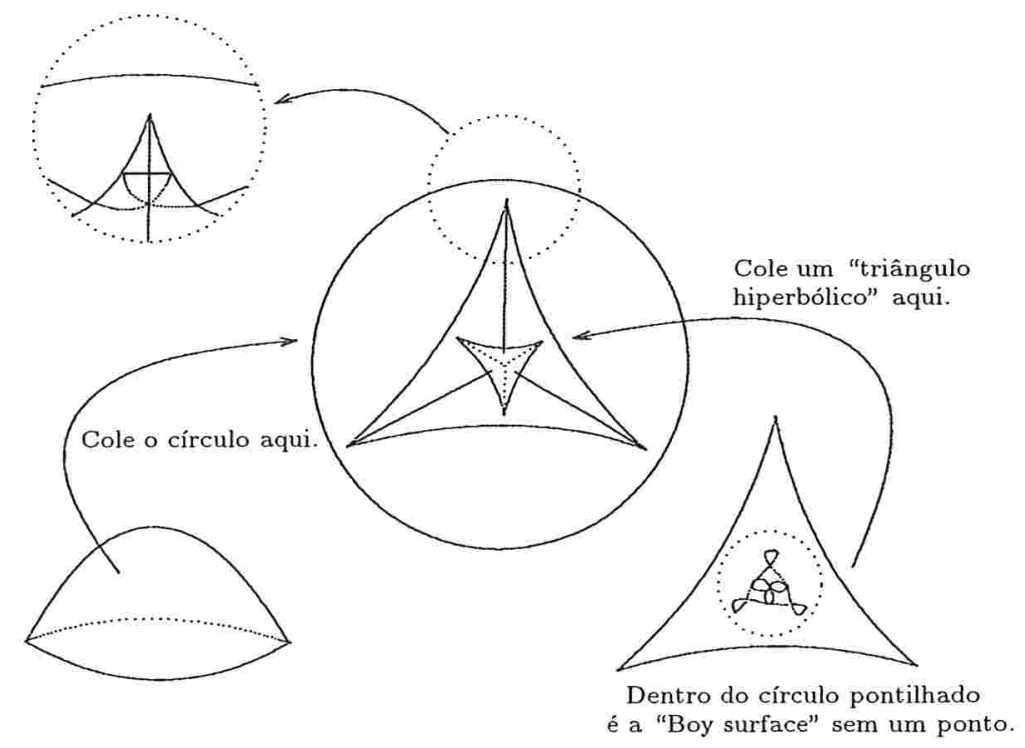

Figura 6.1: imagem de $S\left(f_{1}\right)$. 
Neste caso, temos 2 pontos triplos, sendo que um deles é do tipo $T_{1}$ e 6 rabos de andorinha definidos. Levando-se em consideração que os 6 rabos de andorinha são do mesmo tipo, mas 3 deles têm multiplicidade 1 e os outros 3 têm multiplicidade 2 , pelo Corolário 6.2.7, temos que

$$
\left(\sharp T_{12}+\sharp T_{13}\right) \equiv 1(\bmod 2) \text {. }
$$

Mas como $\sharp T=2, \sharp T_{1}=1$, o outro ponto triplo tem que ser do tipo $T_{12}$ ou $T_{13}$.

Observação 6.2.10 Existe um pequeno erro no [Ko, Theorem 2.1, pg.6] (veja Observação 6.3.10), mas que não invalida o resultado obtido acima.

Exemplo 6.2.11 No [Ko, Theorem 3.1, pg.8], Kobayashi apresenta uma aplicação estável $f_{2}: \mathbb{C P}^{2} \rightarrow \mathbb{R}^{3}$ com as seguintes propriedades:

1) O conjunto dos pontos singulares $S\left(f_{2}\right)$ é difeomorfo à união disjunta $S^{2} \cup \mathbb{R} P^{2}$

2) A aplicação apresenta um círculo de cúspides, algumas regiões de dobras e não tem nenhum rabo de andorinha;

3) O conjunto dos valores singulares $f_{2}\left(S\left(f_{2}\right)\right) \subset \mathbb{R}^{3}$ é a superfície singular que é obtida de um "flying saucer floating" em $D^{3}$ trocando um disco ao redor de um ponto $P$ (como na. Figura 6.2) por uma "Boy surface" $\backslash \operatorname{Int} D^{2}$. 


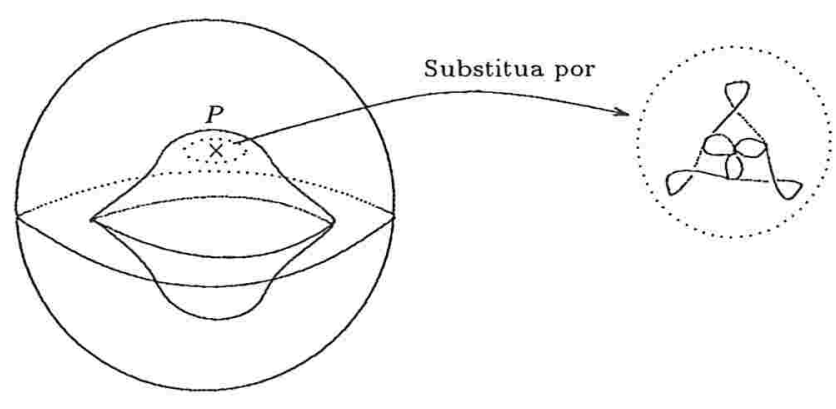

Figura 6.2: imagem de $S\left(f_{2}\right)$.

Analogamente ao Exemplo 6.2.8, $f_{2}$ não possui rabos de andorinha e possui apenas um ponto triplo. Portanto, este tem que ser do tipo $T_{12}$ ou $T_{13}$.

Observação 6.2.12 Como conseqüência do Corolário 6.2.7, temos que se $g$ não possui rabos de andorinha e possui apenas um ponto triplo, este tem que ser do tipo $T_{12}$ ou $T_{13}$. Mais ainda, segundo o Corolário 5.4 de [Sa2], a característica de Euler $\chi\left(N^{4}\right)$ tem que ser ímpar.

\subsection{O índice $\operatorname{Ind}_{f}^{*}(v)$ e o número de singulari- dades}

Nesta seção utilizamos o índice $\operatorname{Ind}_{f}^{\star}(v)$ (Definição 5.3.1) para obter uma fórmula relacionando as singularidades de aplicações $\mathcal{C}^{\infty}$-estáveis $N^{4} \rightarrow \mathbb{R}^{3}$ com certos invariantes topológicos. No que segue, usamos as notações introduzidas na Seção 6.2 para a aplicação $\mathcal{C}^{\infty}$-estável $g: N^{4} \rightarrow \mathbb{R}^{3}$ e a sua triangulação $f: K \rightarrow L$. Mais precisamente, utilizamos a seguinte:

Notação 6.3.1 Sejam $N^{4}$ uma variedade 4-dimensional (orientável ou não) e fechada, $g: N^{4} \rightarrow \mathbb{R}^{3}$ uma aplicação $\mathcal{C}^{\infty}$-estável e $S(g)$ o conjunto singular de $g$. Fixada $f: K \rightarrow L$, uma triangulação de $g$ ([Ve1],[Ve2]), sejam 
$S(f)$ a triangulação de $S(g)$ e $f(S(f))$ a triangulação de $g(S(g))$. Sejam, ainda, $S_{0} \subset N^{4}$ o conjunto das dobras definidas, $S_{1} \subset N^{4}$ o conjunto das dobras indefinidas, $C \subset N^{4}$ o conjunto das cúspides, $W_{0} \subset N^{4}$ o conjunto dos rabos de andorinha definidos, e $W_{1} \subset N^{4}$ o conjunto dos rabos de andorinha indefinidos. Usamos as mesmas notações para os subconjuntos de $K$ correspondentes. Então, definimos como a seguir:

- $D=\left\{r \in g(S(g)): g^{-1}(r) \cap S(g)=g^{-1}(r) \cap S_{0}\right.$ consiste de um ponto $\}$,

- $I=\left\{r \in g(S(g)): g^{-1}(r) \cap S(g)=g^{-1}(r) \cap S_{1}\right.$ consiste de um ponto $\}$,

- $C=\left\{r \in g(S(g)): g^{-1}(r) \cap S(g)=g^{-1}(r) \cap C\right\}$ (Note que o segundo $C$ denota o conjunto das cúspides de $g$ contidas em $N^{4}$. Usamos a mesma notação $C$ para estes dois conjuntos diferentes, pois eles correspondem biunívocamente por $g$ ),

- $F F_{A}=\left\{r \in g(S(g)): g^{-1}(r) \cap S(g)=g^{-1}(r) \cap S_{0}\right.$ consiste de dois pontos\},

- $F F_{B}=\left\{r \in g(S(g)): g^{-1}(r) \cap S(g)\right.$ consiste de dois pontos e $g^{-1}(r) \cap$ $S_{0} \neq \emptyset$ e $\left.g^{-1}(r) \cap S_{1} \neq \emptyset\right\}$

- $F F_{C}=\left\{r \in g(S(g)): g^{-1}(r) \cap S(g)=g^{-1}(r) \cap S_{1}\right.$ consiste de dois pontos\},

- $S W_{D}=\left\{r \in g(S(g)): g^{-1}(r) \cap S(g)=g^{-1}(r) \cap W_{0}\right\}$,

- $S W_{I}=\left\{r \in g(S(g)): g^{-1}(r) \cap S(g)=g^{-1}(r) \cap W_{1}\right\}$,

- $T_{A}=\left\{r \in g(S(g)): g^{-1}(r) \cap S(g)=g^{-1}(r) \cap S_{0}\right.$ consiste de três pontos\},

- $T_{B}=\left\{r \in g(S(g)): g^{-1}(r) \cap S(g)\right.$ consiste de três pontos, $g^{-1}(r) \cap S_{0}$ consiste de dois pontos, e $g^{-1}(r) \cap S_{1}$ consiste de um ponto $\}$, 
- $T_{C}=\left\{r \in g(S(g)): g^{-1}(r) \cap S(g)\right.$ consiste de três pontos, $g^{-1}(r) \cap S_{0}$ consiste de um ponto, e $g^{-1}(r) \cap S_{1}$ consiste de dois pontos\},

- $T_{D}=\left\{r \in g(S(g)): g^{-1}(r) \cap S(g)=g^{-1}(r) \cap S_{1}\right.$ consiste de três pontos\},

- $C F_{A}=\left\{r \in g(S(g)): g^{-1}(r) \cap S(g)\right.$ consiste de dois pontos e $g^{-1}(r) \cap$ $S_{0} \neq \emptyset$ e $\left.g^{-1}(r) \cap C \neq \emptyset\right\}$,

- $C F_{B}=\left\{r \in g(S(g)): g^{-1}(r) \cap S(g)\right.$ consiste de dois pontos e $g^{-1}(r) \cap$ $S_{1} \neq \emptyset$ e $\left.g^{-1}(r) \cap C \neq \emptyset\right\}$

- Usamos as notações $D, I, C, F F_{A}, F F_{B}, F F_{C}, S W_{D}, S W_{I}, T_{A}, T_{B}, T_{C}$, $T_{D}, C F_{A}$ e $C F_{B}$ para os subconjuntos correspondentes de $f(S(f))$ também.

- Para denotar o número de elementos de um conjunto finito $X$, usamos $\sharp X$.

Observação 6.3.2 Os números dos vértices contidos em $f(K) \backslash f(S(f)), D$, $I, C, F F_{A}, F F_{B}$ ou $F F_{C}$ dependem da escolha da triangulação $f: K \rightarrow L$ de $g$. Mas os números $\sharp S W_{D}, \sharp S W_{I}, \sharp T_{A}, \sharp T_{B}, \sharp T_{C}, \sharp T_{D}, \sharp C F_{A}$ e $\sharp C F_{B}$ não.

Iniciamos calculando os índices $\operatorname{Ind}_{f}^{\star}(v)=\sum_{\tau \ni v} \frac{(-1)^{\operatorname{dim} \tau}}{1+\operatorname{dim} \tau}\left(1-\chi\left(f^{-1}\left(b_{\tau}\right)\right)\right)$, onde $b_{\tau}$ é o baricentro de $\tau$ (Definição 5.3.1). Para isso considere: 
$a_{v}^{0} \quad$ o número de arestas de $f(K)$ que contém $v$ e cujos interiores estão contidos em $f(K) \backslash f(S(f))$,

$b_{v}^{0} \quad$ o número de faces de $f(K)$ que contém $v$ e cujos interiores estão contidos em $f(K) \backslash f(S(f))$,

$c_{v}^{0} \quad$ o número de tetraedros de $f(K)$ que contém $v$ e cujos interiores estão contidos em $f(K) \backslash f(S(f))$,

$a_{v}^{1} \quad$ o número de arestas de $f(K)$ que contém $v$ e cujos interiores estão contidos em $D$,

$b_{v}^{1} \quad$ o número de faces de $f(K)$ que contém $v$ e cujos interiores o estão contidos em $D$,

$a_{v}^{-1}$ o número de arestas de $f(K)$ que contém $v$ e cujos interiores estão contidos em $I$,

$b_{v}^{-1} \quad$ o número de faces de $f(K)$ que contém $v$ e cujos interiores estão contidos em $I$,

$I_{v}^{0}=-\frac{a_{v}^{0}}{2}+\frac{b_{v}^{0}}{3}-\frac{c_{v}^{0}}{4}$,

$I_{v}^{-1}=-\frac{a_{v}^{-1}}{2}+\frac{b_{v}^{-1}}{3}$.

Observação 6.3.3 No cálculo dos índices abaixo levamos em consideração que cada componente regular das fibras de $g$ (ou de $f$ ) é homeomorfa a $S^{1}$, e por isso tem característica de Euler nula. No Apêndice D estão enumeradas as características de Euler de todos os tipos de fibras de uma aplicação estável $N^{4} \rightarrow \mathbb{R}^{3}$. Para os vértices $v \in(f(K))^{(0)}$ temos o seguinte:

I) $v \in(f(K))^{(0)} \backslash f(S(f))$.

$$
\begin{aligned}
\operatorname{Ind}_{f}^{\star}(v) & =(1-0)-\frac{a_{v}^{0}}{2}(1-0)+\frac{b_{v}^{0}}{3}(1-0)-\frac{c_{v}^{0}}{4}(1-0) \\
& =1-\frac{a_{v}^{0}}{2}+\frac{b_{v}^{0}}{3}-\frac{c_{v}^{0}}{4} \\
& =1+I_{v}^{0} .
\end{aligned}
$$


II) $v \in(f(K))^{(0)} \cap D$.

$$
\begin{aligned}
\operatorname{Ind}_{f}^{\star}(v) & =(1-1)-\frac{a_{v}^{0}}{2}(1-0)-\frac{a_{v}^{1}}{2}(1-1)+\frac{b_{v}^{0}}{3}(1-0)+\frac{b_{v}^{1}}{3}(1-1) \\
& -\frac{c_{v}^{0}}{4}(1-0) \\
& =-\frac{a_{v}^{0}}{2}+\frac{b_{v}^{0}}{3}-\frac{c_{v}^{0}}{4} \\
& =I_{v}^{0} .
\end{aligned}
$$

III) $v \in(f(K))^{(0)} \cap I$.

$$
\begin{aligned}
\operatorname{Ind}_{f}^{*}(v) & =(1+1)-\frac{a_{v}^{0}}{2}(1-0)-\frac{a_{v}^{-1}}{2}(1+1)+\frac{b_{v}^{0}}{3}(1-0)+\frac{b_{v}^{-1}}{3}(1+1) \\
& -\frac{c_{v}^{0}}{4}(1-0) \\
& =\left(-\frac{a_{v}^{0}}{2}+\frac{b_{v}^{0}}{3}-\frac{c_{v}^{0}}{4}\right)+2\left(1-\frac{a_{v}^{-1}}{2}+\frac{b_{v}^{-1}}{3}\right) \\
& =I_{v}^{0}+2\left(1+I_{v}^{-1}\right) .
\end{aligned}
$$

IV) $v \in(f(K))^{(0)} \cap C$.

$$
\begin{aligned}
\operatorname{Ind}_{f}^{\star}(v) & =(1-0)-\frac{a_{v}^{0}}{2}(1-0)-\frac{a_{v}^{1}}{2}(1-1)-\frac{a_{v}^{-1}}{2}(1+1)-\frac{2}{2}(1-0) \\
& +\frac{b_{v}^{0}}{3}(1-0)+\frac{b_{v}^{1}}{3}(1-1)+\frac{b_{v}^{-1}}{3}(1+1)-\frac{c_{v}^{0}}{4}(1-0) \\
& =\left(-\frac{a_{v}^{0}}{2}+\frac{b_{v}^{0}}{3}-\frac{c_{v}^{0}}{4}\right)+2\left(-\frac{a_{v}^{-1}}{2}+\frac{b_{v}^{-1}}{3}\right) \\
& =I_{v}^{0}+2 I_{v}^{-1} .
\end{aligned}
$$

V) Pontos duplos (dobra + dobra).

a) $v \in(f(K))^{(0)} \cap F F_{A}$.

$$
\begin{aligned}
\operatorname{Ind}_{f}^{\star}(v) & =(1-2)-\frac{a_{v}^{0}}{2}(1-0)-\frac{a_{v}^{1}}{2}(1-1)-\frac{2}{2}(1-2)+\frac{b_{v}^{0}}{3}(1-0) \\
& +\frac{b_{v}^{1}}{3}(1-1)-\frac{c_{v}^{0}}{4}(1-0) \\
& =-\frac{a_{v}^{0}}{2}+\frac{b_{v}^{0}}{3}-\frac{c_{v}^{0}}{4} \\
& =I_{v}^{0} .
\end{aligned}
$$


b) $v \in(f(K))^{(0)} \cap F F_{B}$.

$$
\begin{aligned}
\operatorname{Ind}_{f}^{\star}(v) & =(1-0)-\frac{a_{v}^{0}}{2}(1-0)-\frac{a_{v}^{1}}{2}(1-1)-\frac{a_{v}^{-1}}{2}(1+1)-\frac{2}{2}(1-0) \\
& +\frac{b_{v}^{0}}{3}(1-0)+\frac{b_{v}^{1}}{3}(1-1)+\frac{b_{v}^{-1}}{3}(1+1)-\frac{c_{v}^{0}}{4}(1-0) \\
& =\left(-\frac{a_{v}^{0}}{2}+\frac{b_{v}^{0}}{3}-\frac{c_{v}^{0}}{4}\right)+2\left(-\frac{a_{v}^{-1}}{2}+\frac{b_{v}^{-1}}{3}\right) \\
& =I_{v}^{0}+2 I_{v}^{-1} .
\end{aligned}
$$

c) $v \in(f(K))^{(0)} \cap F F_{C}$.

$$
\begin{aligned}
\operatorname{Ind}_{f}^{\star}(v) & =(1+2)-\frac{a_{v}^{0}}{2}(1-0)-\frac{a_{v}^{-1}}{2}(1+1)-\frac{2}{2}(1+2)+\frac{b_{v}^{0}}{3}(1-0) \\
& +\frac{b_{v}^{-1}}{3}(1+1)-\frac{c_{v}^{0}}{4}(1-0) \\
& =\left(-\frac{a_{v}^{0}}{2}+\frac{b_{v}^{0}}{3}-\frac{c_{v}^{0}}{4}\right)+2\left(-\frac{a_{v}^{-1}}{2}+\frac{b_{v}^{-1}}{3}\right) \\
& =I_{v}^{0}+2 I_{v}^{-1} .
\end{aligned}
$$

VI) Pontos triplos.

a) $v \in T_{A}$.

$$
\begin{aligned}
\operatorname{Ind}_{f}^{\star}(v) & =(1-3)-\frac{a_{v}^{0}}{2}(1-0)-\frac{a_{v}^{1}}{2}(1-1)-\frac{6}{2}(1-2)+\frac{b_{v}^{0}}{3}(1-0) \\
& +\frac{b_{v}^{1}}{3}(1-1)-\frac{c_{v}^{0}}{4}(1-0) \\
& =\left(-\frac{a_{v}^{0}}{2}+\frac{b_{v}^{0}}{3}-\frac{c_{v}^{0}}{4}\right)+1 \\
& =I_{v}^{0}+1 .
\end{aligned}
$$


b) $v \in T_{B}$.

$$
\begin{aligned}
\operatorname{Ind}_{f}^{\star}(v) & =(1-1)-\frac{a_{v}^{0}}{2}(1-0)-\frac{a_{v}^{1}}{2}(1-1)-\frac{a_{v}^{-1}}{2}(1+1)-\frac{4}{2}(1-0) \\
& -\frac{2}{2}(1-2)+\frac{b_{v}^{0}}{3}(1-0)+\frac{b_{v}^{1}}{3}(1-1)+\frac{b_{v}^{-1}}{3}(1+1)-\frac{c_{v}^{0}}{4}(1-0) \\
& =\left(-\frac{a_{v}^{0}}{2}+\frac{b_{v}^{0}}{3}-\frac{c_{v}^{0}}{4}\right)+2\left(-\frac{a_{v}^{-1}}{2}+\frac{b_{v}^{-1}}{3}\right)-1 \\
& =I_{v}^{0}+2 I_{v}^{-1}-1 .
\end{aligned}
$$

c) $v \in T_{C}$.

$$
\begin{aligned}
\operatorname{Ind}_{f}^{\star}(v) & =(1+1)-\frac{a_{v}^{0}}{2}(1-0)-\frac{a_{v}^{1}}{2}(1-1)-\frac{a_{v}^{-1}}{2}(1+1)-\frac{4}{2}(1-0) \\
& -\frac{2}{2}(1+2)+\frac{b_{v}^{0}}{3}(1-0)+\frac{b_{v}^{1}}{3}(1-1)+\frac{b_{v}^{-1}}{3}(1+1)-\frac{c_{v}^{0}}{4}(1-0) \\
& =\left(-\frac{a_{v}^{0}}{2}+\frac{b_{v}^{0}}{3}-\frac{c_{v}^{0}}{4}\right)+2\left(-\frac{a_{v}^{-1}}{2}+\frac{b_{v}^{-1}}{3}\right)-3 \\
& =I_{v}^{0}+2 I_{v}^{-1}-3 .
\end{aligned}
$$

d) $v \in T_{D}$.

$$
\begin{aligned}
\operatorname{Ind}_{f}^{\star}(v) & =(1+3)-\frac{a_{v}^{0}}{2}(1-0)-\frac{a_{v}^{-1}}{2}(1+1)-\frac{6}{2}(1+2)+\frac{b_{v}^{0}}{3}(1-0) \\
& +\frac{b_{v}^{-1}}{3}(1+1)-\frac{c_{v}^{0}}{4}(1-0) \\
& =\left(-\frac{a_{v}^{0}}{2}+\frac{b_{v}^{0}}{3}-\frac{c_{v}^{0}}{4}\right)+2\left(-\frac{a_{v}^{-1}}{2}+\frac{b_{v}^{-1}}{3}\right)-5 \\
& =I_{v}^{0}+2 I_{v}^{-1}-5 .
\end{aligned}
$$

VII) Pontos duplos (dobra + cúspide). 
a) $v \in C F_{A}$.

$$
\begin{aligned}
\operatorname{Ind}_{f}^{\star}(v) & =(1-1)-\frac{a_{v}^{0}}{2}(1-0)-\frac{a_{v}^{1}}{2}(1-1)-\frac{a_{v}^{-1}}{2}(1+1)-\frac{3}{2}(1-0) \\
& -\frac{1}{2}(1-2)+\frac{b_{v}^{0}}{3}(1-0)+\frac{b_{v}^{1}}{3}(1-1)+\frac{b_{v}^{-1}}{3}(1+1)-\frac{c_{v}^{0}}{4}(1-0) \\
& =\left(-\frac{a_{v}^{0}}{2}+\frac{b_{v}^{0}}{3}-\frac{c_{v}^{0}}{4}\right)+2\left(-\frac{a_{v}^{-1}}{2}+\frac{b_{v}^{-1}}{3}\right)-1 \\
& =I_{v}^{0}+2 I_{v}^{-1}-1 .
\end{aligned}
$$

b) $v \in C F_{B}$.

$$
\begin{aligned}
\operatorname{Ind}_{f}^{\star}(v) & =(1+1)-\frac{a_{v}^{0}}{2}(1-0)-\frac{a_{v}^{1}}{2}(1-1)-\frac{a_{v}^{-1}}{2}(1+1)-\frac{3}{2}(1-0) \\
& -\frac{1}{2}(1+2)+\frac{b_{v}^{0}}{3}(1-0)+\frac{b_{v}^{1}}{3}(1-1)+\frac{b_{v}^{-1}}{3}(1+1)-\frac{c_{v}^{0}}{4}(1-0) \\
& =\left(-\frac{a_{v}^{0}}{2}+\frac{b_{v}^{0}}{3}-\frac{c_{v}^{0}}{4}\right)+2\left(-\frac{a_{v}^{-1}}{2}+\frac{b_{v}^{-1}}{3}\right)-1 \\
& =I_{v}^{0}+2 I_{v}^{-1}-1 .
\end{aligned}
$$

VIII) Rabos de andorinha.

a) $v \in S W_{D}$.

$$
\begin{aligned}
\operatorname{Ind}_{f}^{\star}(v) & =(1-1)-\frac{a_{v}^{0}}{2}(1-0)-\frac{a_{v}^{1}}{2}(1-1)-\frac{a_{v}^{-1}}{2}(1+1)-\frac{2}{2}(1-0) \\
& -\frac{1}{2}(1-2)+\frac{b_{v}^{0}}{3}(1-0)+\frac{b_{v}^{1}}{3}(1-1)+\frac{b_{v}^{-1}}{3}(1+1)-\frac{c_{v}^{0}}{4}(1-0) \\
& =\left(-\frac{a_{v}^{0}}{2}+\frac{b_{v}^{0}}{3}-\frac{c_{v}^{0}}{4}\right)+2\left(-\frac{a_{v}^{-1}}{2}+\frac{b_{v}^{-1}}{3}\right)-\frac{1}{2} \\
& =I_{v}^{0}+2 I_{v}^{-1}-\frac{1}{2} .
\end{aligned}
$$


b) $v \in S W_{I}$.

$$
\begin{aligned}
\operatorname{Ind}_{f}^{\star}(v) & =(1+1)-\frac{a_{v}^{0}}{2}(1-0)-\frac{a_{v}^{1}}{2}(1-1)-\frac{a_{v}^{-1}}{2}(1+1)-\frac{2}{2}(1-0) \\
& -\frac{1}{2}(1+2)+\frac{b_{v}^{0}}{3}(1-0)+\frac{b_{v}^{1}}{3}(1-1)+\frac{b_{v}^{-1}}{3}(1+1)-\frac{c_{v}^{0}}{4}(1-0) \\
& =\left(-\frac{a_{v}^{0}}{2}+\frac{b_{v}^{0}}{3}-\frac{c_{v}^{0}}{4}\right)+2\left(-\frac{a_{v}^{-1}}{2}+\frac{b_{v}^{-1}}{3}\right)-\frac{1}{2} \\
& =I_{v}^{0}+2 I_{v}^{-1}-\frac{1}{2} .
\end{aligned}
$$

Teorema 6.3.4 Seja $g: N^{4} \rightarrow \mathbb{R}^{3}$ uma aplicação $\mathcal{C}^{\infty}$-estável, onde $N^{4}$ é uma 4-variedade fechada. Então:

$\chi(g(S(g)))-\chi\left(N^{4}\right)-2 \chi(\bar{I})=\sharp T_{A}+\sharp T_{B}+\sharp T_{C}-\sharp T_{D}+\sharp C F_{B}-\frac{\sharp S W_{D}-\sharp S W_{I}}{2}$, onde $I$ é o conjunto dos pontos $q \in g(S(g))$ tais que $g^{-1}(q) \cap S(g)$ consiste exatamente de um ponto de dobra indefinida.

\section{Demonstração:}

Seja $f: K \rightarrow L$ uma triangulação de $g$. Então:

$$
\begin{aligned}
& \chi(f(K))-\chi(K)=\sum_{v \in(f(K))^{(0)}} \operatorname{Ind}_{f}^{\star}(v) \\
& =\sum_{v \in(f(K))^{(0)} \backslash f(S(f))} \operatorname{Ind}_{f}^{\star}(v)+\sum_{v \in(f(K))^{(0)} \cap D} \operatorname{Ind}_{f}^{\star}(v)+\sum_{v \in(f(K))^{(0)} \cap I} \operatorname{Ind}_{f}^{\star}(v) \\
& +\sum_{v \in(f(K))^{(0)} \cap C} \operatorname{Ind}_{f}^{\star}(v)+\sum_{v \in(f(K))^{(0)} \cap F F_{A}} \operatorname{Ind}_{f}^{\star}(v)+\sum_{v \in(f(K))^{(0)} \cap F F_{B}} \operatorname{Ind}_{f}^{\star}(v) \\
& +\sum_{v \in(f(K))^{(0)} \cap F F_{C}} \operatorname{Ind}_{f}^{\star}(v)+\sum_{v \in T_{A}} \operatorname{Ind}_{f}^{*}(v)+\sum_{v \in T_{B}} \operatorname{Ind}_{f}^{\star}(v) \\
& +\quad \sum_{v \in T_{C}} \operatorname{Ind}_{f}^{\star}(v) \quad+\sum_{v \in T_{D}} \operatorname{Ind}_{f}^{\star}(v)+\sum_{v \in C F_{A}} \operatorname{Ind}_{f}^{\star}(v) \\
& +\quad \sum_{v \in C F_{B}} \operatorname{Ind}_{f}^{*}(v)+\sum_{v \in S W_{D}} \operatorname{Ind}_{f}^{*}(v)+\sum_{v \in S W_{I}} \operatorname{Ind}_{f}^{*}(v)
\end{aligned}
$$




$$
\begin{aligned}
& =\sum_{v \in(f(K))^{(0)} \backslash f(S(f))}\left(1+I_{v}^{0}\right)+\sum_{v \in(f(K))^{(0)} \cap D}\left(I_{v}^{0}\right)+\sum_{v \in(f(K))^{(0)} \cap I}\left(I_{v}^{0}+2\left(1+I_{v}^{-1}\right)\right) \\
& +\sum_{v \in(f(K))^{(0)} \cap C}\left(I_{v}^{0}+2 I_{v}^{-1}\right)+\sum_{v \in(f(K))^{(0)} \cap F F_{A}}\left(I_{v}^{0}\right)+\sum_{v \in(f(K))^{(0)} \cap F F_{B}}\left(I_{v}^{0}+2 I_{v}^{-1}\right) \\
& +\sum_{v \in(f(K))^{(0)} \cap F F_{C}}\left(I_{v}^{0}+2 I_{v}^{-1}\right)+\sum_{v \in T_{A}}\left(I_{v}^{0}+1\right)+\sum_{v \in T_{B}}\left(I_{v}^{0}+2 I_{v}^{-1}-1\right) \\
& +\sum_{v \in T_{C}}\left(I_{v}^{0}+2 I_{v}^{-1}-3\right)+\sum_{v \in T_{D}}\left(I_{v}^{0}+2 I_{v}^{-1}-5\right)+\sum_{v \in C F_{A}}\left(I_{v}^{0}+2 I_{v}^{-1}-1\right) \\
& +\sum_{v \in C F_{B}}\left(I_{v}^{0}+2 I_{v}^{-1}-1\right)+\sum_{v \in S W_{D}}\left(I_{v}^{0}+2 I_{v}^{-1}-\frac{1}{2}\right)+\sum_{v \in S W_{I}}\left(I_{v}^{0}+2 I_{v}^{-1}-\frac{1}{2}\right) .
\end{aligned}
$$

Seja $X=C \cup F F_{B} \cup F F_{C} \cup T_{B} \cup T_{C} \cup T_{D} \cup C F_{A} \cup C F_{B} \cup S W_{D} \cup S W_{I}$, então, temos:

$$
\begin{aligned}
\chi(f(K))-\chi(K) & =\sum_{v \in(f(K))^{(0)} \backslash f(S(f))}\left(1+I_{v}^{0}\right)+\sum_{v \in(f(S(f)))^{(0)}} I_{v}^{0} \\
& +2 \sum_{v \in(f(K)))^{(0)} \cap I}\left(1+I_{v}^{-1}\right)+2 \sum_{v \in X^{(0)}} I_{v}^{-1}+\sharp T_{A}-\sharp T_{B}-3 \sharp T_{C} \\
& -5 \sharp T_{D}-\sharp C F_{A}-\sharp C F_{B}-\frac{\sharp S W_{D}}{2}-\frac{\sharp S W_{I}}{2} \\
& =\sum_{v \in(f(K))^{(0)} \backslash f(S(f))}\left(1+I_{v}^{0}\right)+\sum_{v \in(f(S(f)))^{(0)}} I_{v}^{0} \\
& +2\left(\sum_{v \in(f(K))^{(0)} \cap I}\left(1+I_{v}^{-1}\right)+\sum_{v \in X(0)} I_{v}^{-1}\right) \\
& +\sharp T_{A}-\sharp T_{B}-3 \sharp T_{C}-5 \sharp T_{D}-\sharp C F_{A}-\sharp C F_{B} \\
& -\frac{\sharp S W_{D}}{2}-\frac{\sharp S W_{I}}{2}
\end{aligned}
$$




$$
\begin{aligned}
& =\chi_{c}(f(K) \backslash f(S(f)))+2 \chi_{c}(I)+\sharp T_{A}-\sharp T_{B}-3 \sharp T_{C}-5 \sharp T_{D} \\
& -\sharp C F_{A}-\sharp C F_{B}-\frac{\sharp S W_{D}}{2}-\frac{\sharp S W_{I}}{2} .
\end{aligned}
$$

Observemos que $\bar{I}-I=X$, então $\chi_{c}(I)=\chi(\bar{I})-\chi(X)$ e lembremos que $\chi\left(f\left(K^{\prime}\right)\right)=\chi_{c}\left(f\left(K^{r}\right) \backslash f(S(f))\right)+\chi(f(S(f)))$; portanto,

$$
\begin{aligned}
\chi( & (S(f)))-\chi(K) \\
& =2 \chi_{c}(I)+\sharp T_{A}-\sharp T_{B}-3 \sharp T_{C}-5 \sharp T_{D}-\sharp C F_{A}-\sharp C F_{B}-\frac{\sharp S W_{D}}{2}-\frac{\sharp S W_{I}}{2} \\
& =2(\chi(\bar{I})-\chi(X))+\sharp T_{A}-\sharp T_{B}-3 \sharp T_{C}-5 \sharp T_{D}-\sharp C F_{A}-\sharp C F_{B}-\frac{\sharp S W_{D}}{2}-\frac{\sharp S W_{I}}{2} \\
& =2 \chi(\bar{I})-2 \chi(X)+\sharp T_{A}-\sharp T_{B}-3 \sharp T_{C}-5 \sharp T_{D}-\sharp C F_{A}-\sharp C F_{B}-\frac{\sharp S W_{D}}{2}-\frac{\sharp S W_{I}}{2} .
\end{aligned}
$$

Notemos que $\chi(X)=\sum_{v \in X^{(0)}}\left(1-\frac{d_{v}}{2}\right)$, onde $d_{v}$ é o número de arestas de $X$ que tem $v$ como um de seus vértices. Assim,

$$
\chi(X)=-\sharp T_{B}-2 \sharp T_{C}-2 \sharp T_{D}-\frac{1}{2} \sharp C F_{A}-\sharp C F_{B}-\frac{1}{2} \sharp S W_{I} .
$$

Portanto,

$$
\begin{aligned}
\chi( & (S(f)))-\chi(K)=2 \chi(\bar{I})-2\left(-\sharp T_{B}-2 \sharp T_{C}-2 \sharp T_{D}-\frac{1}{2} \sharp C F_{A}-\sharp C F_{B}-\frac{1}{2} \sharp S W_{I}\right) \\
& +\sharp T_{A}-\sharp T_{B}-3 \sharp T_{C}-5 \sharp T_{D}-\sharp C F_{A}-\sharp C F_{B}-\frac{\sharp S W_{D}}{2}-\frac{\sharp S W_{I}}{2} \\
& =2 \chi(\bar{I})+2 \sharp T_{B}+4 \sharp T_{C}+4 \sharp T_{D}+\sharp C F_{A}+2 \sharp C F_{B}+\sharp S W_{I}+\sharp T_{A}-\sharp T_{B}-3 \sharp T_{C} \\
& -5 \sharp T_{D}-\sharp C F_{A}-\sharp C F_{B}-\frac{\sharp S W_{D}}{2}-\frac{\sharp S W_{I}}{2}
\end{aligned}
$$


$=2 \chi(\bar{I})+\sharp T_{A}+\sharp T_{B}+\sharp T_{C}-\sharp T_{D}+\sharp C F_{B}-\frac{\sharp S W_{D}-\sharp S W_{I}}{2}$.

Ou seja,

$\chi(g(S(g)))-\chi\left(N^{4}\right)-2 \chi(\bar{I})=\sharp T_{A}+\sharp T_{B}+\sharp T_{C}-\sharp T_{D}+\sharp C F_{B}-\frac{\sharp S W_{D}-\sharp S W_{I}}{2}$.

Corolário 6.3.5 Nas hipóteses acima, $\sharp S W_{D} \equiv \sharp S W_{I}(\bmod 2)$.

\section{Demonstração:}

Do Teorema 6.3.4, temos que:

$\chi(g(S(g)))-\chi\left(N^{4}\right)-2 \chi(\bar{I})=\sharp T_{A}+\sharp T_{B}+\sharp T_{C}-\sharp T_{D}+\sharp C F_{B}-\frac{\sharp S W_{D}}{2}+\frac{\sharp S W_{I}}{2}$.

Ou seja,

$\sharp S W_{I}=\sharp S W_{D}+2\left(\chi(g(S(g)))-\chi(N)-2 \chi(\bar{I})-\sharp T_{A}-\sharp T_{B}-\sharp T_{C}+\sharp T_{D}\right.$

$\sharp S W_{D} \equiv \sharp S W_{I}(\bmod 2)$.

$\left.-\sharp C F_{B}\right)$.

Corolário 6.3.6 Nas hipóteses acima, temos que

$$
\chi(S(g))-\chi\left(N^{4}\right)=2 \chi(\bar{I})-2 \sharp T_{D}+\sharp C F_{B}-\sharp S W_{D} .
$$

\section{Demonstração:}

Seja $g: N^{4} \rightarrow \mathbb{R}^{3}$ uma aplicação $\mathcal{C}^{\infty}$-estável, onde $N^{4}$ é uma variedade fechada. O conjunto singular $S(g) \subset N^{4}$ é uma subvariedade fechada de dimensão 2 de $N^{4}$. A aplicação $\tilde{g}=\left.g\right|_{S(g)}: S(g) \rightarrow \mathbb{R}^{3}$ satisfaz as condições do [IM2, Theorem 3.1], portanto temos a seguinte relação:

$$
\chi(g(S(g)))-\chi(S(g))=\sharp T+\frac{\sharp C C}{2},
$$

onde $\sharp T$ é o número de pontos triplos e $\sharp C C$ é o número de capuzes cruzados de $\tilde{g}$. Como os capuzes cruzados de $\tilde{g}$ estão em correspondência biunívoca 
com os rabos de andorinha de $g$, podemos reescrever a equação (6.5) da seguinte forma:

$$
\chi(g(S(g)))-\chi(S(g))=\sharp T+\frac{\sharp S W}{2},
$$

onde $\sharp S W$ é o número de rabos de andorinha de $g$.

Ainda mais, pela Observação 6.1.2 sabemos que:

$$
\sharp T=\sharp T_{A}+\sharp T_{B}+\sharp T_{C}+\sharp T_{D} \quad \text { e } \sharp S W=\sharp S W_{D}+\sharp S W_{I} \text {. }
$$

Portanto, a equação (6.6) fica assim:

$$
\chi(g(S(g)))-\chi(S(g))=\sharp T_{A}+\sharp T_{B}+\sharp T_{C}+\sharp T_{D}+\frac{\sharp S W_{D}}{2}+\frac{\sharp S W_{I}}{2} .
$$

Por outro lado, pelo Teorema 6.3.4, sabemos que:

$$
\chi(g(S(g)))-\chi\left(N^{4}\right)=2 \chi(\bar{I})+\sharp T_{A}+\sharp T_{B}+\sharp T_{C}-\sharp T_{D}+\sharp C F_{B}-\frac{\sharp S W_{D}}{2}+\frac{\sharp S W_{I}}{2} .
$$

De (6.8)-(6.7) obtemos:

$$
\chi(S(g))-\chi\left(N^{4}\right)=2 \chi(\bar{I})-2 \sharp T_{D}+\sharp C F_{B}-\sharp S W_{D} .
$$

Corolário 6.3.7 Nas hipóteses acima, temos que $\sharp C F_{B}-\sharp S W_{D} \equiv 0(\bmod 2)$.

\section{Demonstração:}

Segundo o [Sa2, Corollary 5.2], temos que $\chi\left(N^{4}\right) \equiv \chi(S(g))(\bmod 2)$ e pelo Corolário 6.3.6 sabemos que

$$
\chi(S(g))-\chi\left(N^{4}\right)=2 \chi(\bar{I})-2 \sharp T_{D}+\sharp C F_{B}-\sharp S W_{D} .
$$

Portanto,

$$
\sharp C F_{B}-\sharp S W_{D} \equiv 0(\bmod 2) \text {. }
$$


Exemplo 6.3.8 Considere a aplicação $f: \mathbb{C P} P^{2} \sharp 2 \overline{\mathscr{C P}} P^{2} \rightarrow \mathbb{R}^{3}$ como no Exemplo 6.2.8.

Neste caso, o ponto triplo é do tipo $T_{D}, \chi(f(S(f)))=8, \chi\left(\mathscr{C P} P^{2} \sharp 2 \overline{\mathscr{C} P^{2}}\right)=$ $5, \sharp T_{A}=\sharp T_{B}=\sharp T_{C}=0, \sharp T_{D}=1, \sharp C F_{B}=0$ e $\sharp S W_{D}=\sharp S W_{I}=0$.

Segundo o Teorema 6.3.4 temos que

$$
\begin{array}{r}
\chi(f(S(f)))-\chi\left(C P^{2} \sharp 2 \overline{C P^{2}}\right)-2 \chi(\bar{I})=\sharp T_{A}+\sharp T_{B}+\sharp T_{C}-\sharp T_{D}+\sharp C F_{B}-\frac{\sharp S W_{D}}{2} \\
+\frac{\sharp S W_{I}}{2} .
\end{array}
$$

Ou seja, $8-5-2 \chi(\bar{I})=0+0+0-1+0-0+0$. Portanto, $\chi(\bar{I})=2$.

Pelo Corolário 6.3.6, segue que $\chi(S(g))-5=4-2+0-0$, ou seja, $\chi(S(g))=7$.

Fazendo cálculos explícitos verifica-se que realmente $\chi(S(g))=7$, portanto vale a fórmula do Teorema 6.3.4.

Exemplo 6.3.9 Considere a aplicação estável $f_{2}: \mathbb{C} P^{2} \rightarrow \mathbb{R}^{3}$ do Exemplo 6.2.11.

Neste caso, temos que $\chi\left(f_{2}\left(S\left(f_{2}\right)\right)\right)=4, \chi\left(\mathscr{C} P^{2}\right)=3, \chi(\bar{I})=1, \sharp T_{A}=$ $\sharp T_{B}=\sharp T_{C}=0, \sharp T_{D}=1, \sharp C F_{B}=0$ e $\sharp S W_{D}=\sharp S W_{I}=0$. Aplicando a fórmula do Teorema 6.3.4 temos

$$
4-3-2(1)=0+0+0-1+0-0+0
$$

ou seja, vale o Teorema 6.3.4.

Observação 6.3.10 Infelizmente, existe uma pequena falha no [Ko, Theorem 2.1, pg.6]. Neste teorema Kobayashi afirma que existe uma aplicação estável $f_{1}: \mathscr{C} P^{2} \rightarrow \mathbb{R}^{3}$ que está definida no Exemplo 6.2.9.

Calculando as características de Euler obtemos que $\chi\left(f_{1}\left(S\left(f_{1}\right)\right)\right)=4$, $\chi\left(\mathscr{C} P^{2}\right)=3, \chi(\bar{I})=2$. Temos ainda que $\sharp T_{B}=\sharp T_{C}=0, \sharp T_{A}=\sharp T_{D}=1$, $\sharp C F_{B}=0, \sharp S W_{D}=6$ e $\sharp S W_{I}=0$. 
Do Corolário 6.3.6 segue que

$$
\begin{aligned}
\chi\left(S\left(f_{1}\right)\right) & =\chi\left(C P^{2}\right)+2 \chi(\bar{I})-2 \sharp T_{D}+\sharp C F_{B}-\sharp S W_{D} \\
& =3+2(2)-2(1)+0-6 \\
& =-1
\end{aligned}
$$

Mas, $\chi\left(\mathbb{R P} P^{2}\right)=1 \neq-1=\chi\left(S\left(f_{1}\right)\right)$.

É possível construir uma função de Morse $h: S\left(f_{1}\right) \rightarrow \mathbb{R}$ que tem 1 ponto crítico de índice 0,1 ponto crítico de índice 2 , e 3 pontos críticos de índice 1. Isto também significa que $\chi\left(S\left(f_{1}\right)\right)=1-3+1=-1$. Ou seja, $S\left(f_{1}\right)$ é difeomorfo a soma conexa de três cópias de $\mathbb{R} P^{2}$ e não apenas uma cópia de $\mathbb{R} P^{2}$ como está no enunciado do [Ko, Theorem 3.1, pg.6]. 


\section{Apêndice}

Neste apêndice apresentamos algumas figuras que nos auxiliaram no cálculo dos índices dos Capítulos 5 e 6. Elas consistem basicamente das componentes conexas das fibras de pontos pertencentes ao conjunto dos valores singulares de $g: N^{n} \rightarrow \mathbb{R}^{p}$ interceptado pelo conjunto singular $S(g)$ e, também, da característica de Euler destas fibras. As figuras em si representam localmente a imagem do conjunto singular $g(S(g))$ e dentro das figuras coloridas estão as componentes conexas das fibras ou a característica de Euler correspondente. As nomenclaturas utilizadas nos apêndices A, B, C e D estão definidas nas Observações 5.1.4, 5.3.2, 6.2.1 e 6.3.1, respectivamente. 


\section{Apêndice A}

\section{As componentes conexas das fibras de $N^{3} \rightarrow \mathbb{R}^{2}$}

Nas figuras seguintes estão representadas as componentes das fibras. Essa classificação pode ser encontrada em [KLP] e [Le].

Os retângulos azuis indicam as componentes das fibras dos pontos isolados $D D, D I, I I$ ou $C$.

Os losangos verdes indicam as componentes das fibras dos pontos sobre as curvas $D$ ou $I$.

Os retângulos amarelos indicam as componentes das fibras dos valores regulares.

Estamos assumindo que $N^{3}$ é uma variedade diferenciável fechada e orientável. 
Componentes conexas das fibras

\section{Ponto regular}

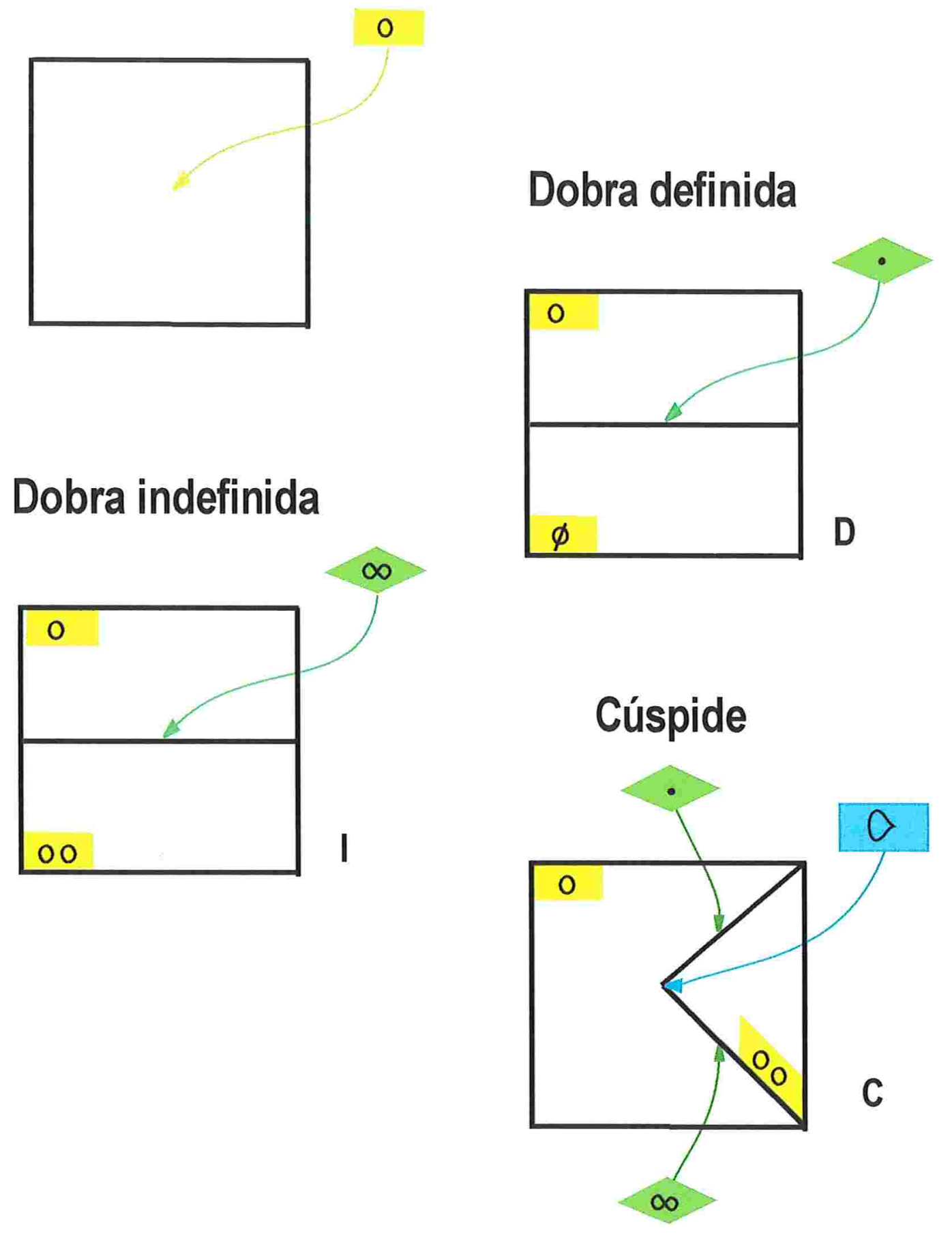




\section{Componentes conexas das fibras}

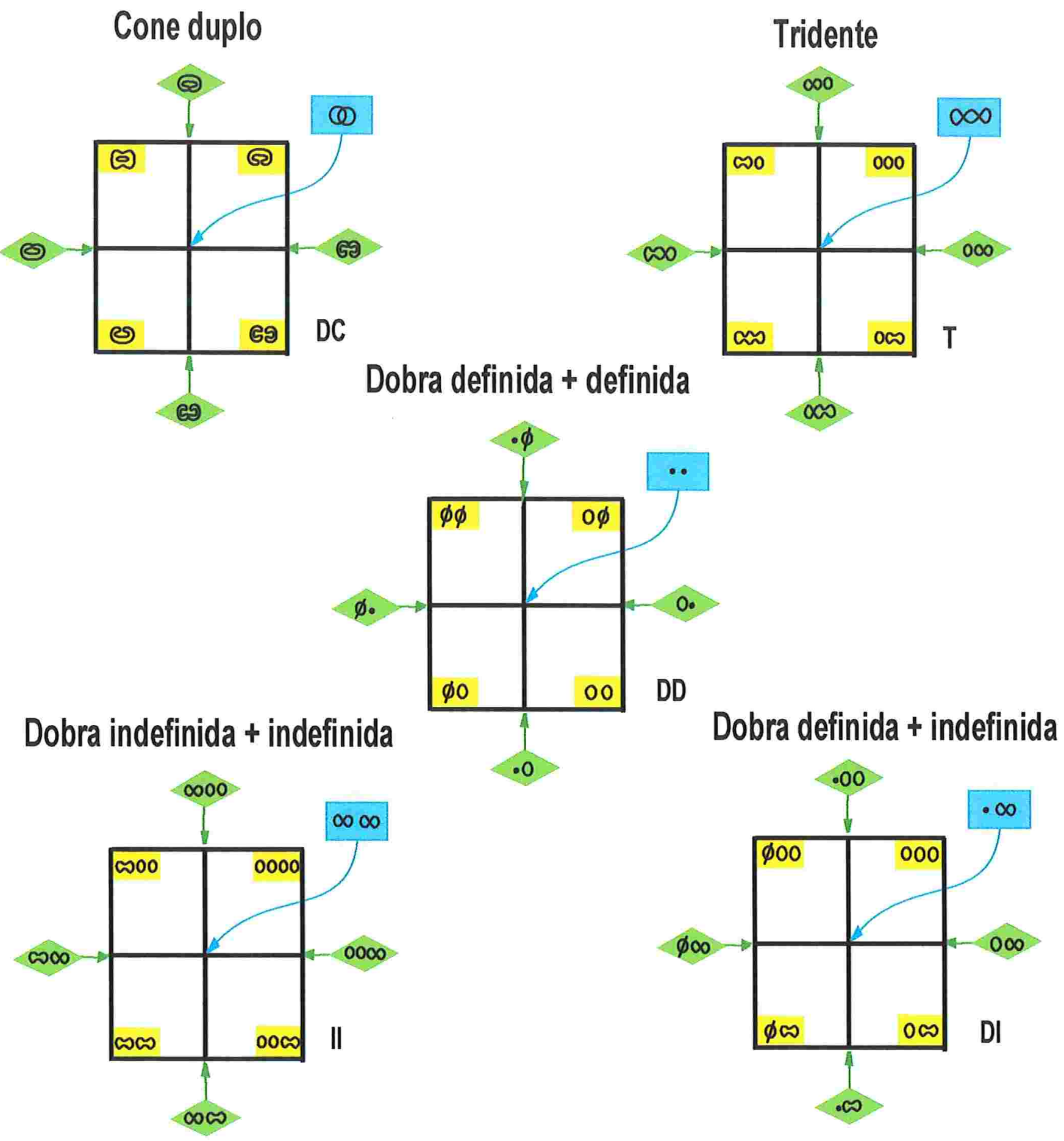




\section{Apêndice B}

\section{As características de Euler das fibras de $N^{3} \rightarrow \mathbb{R}^{2}$}

Os números, que aparecem nas figuras verdes e amarelas desta seção, indicam os valores das características de Euler das fibras que foram calculadas utilizando as figuras do Apêndice A, lembrando que $N^{3}$ é uma variedade diferenciável, fechada e orientável. Eles são necessários para o cálculo dos índices da Observação 5.3.4. 


\section{Característica de Euler das fibras}

\section{Ponto regular}

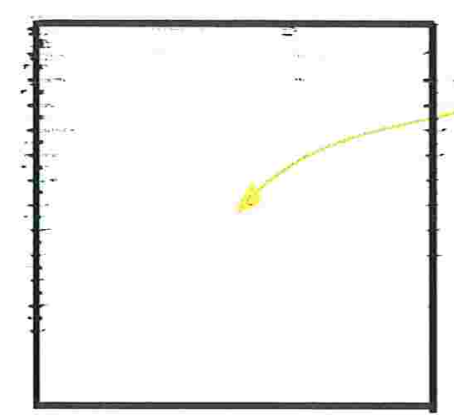

Dobra definida

Dobra indefinida
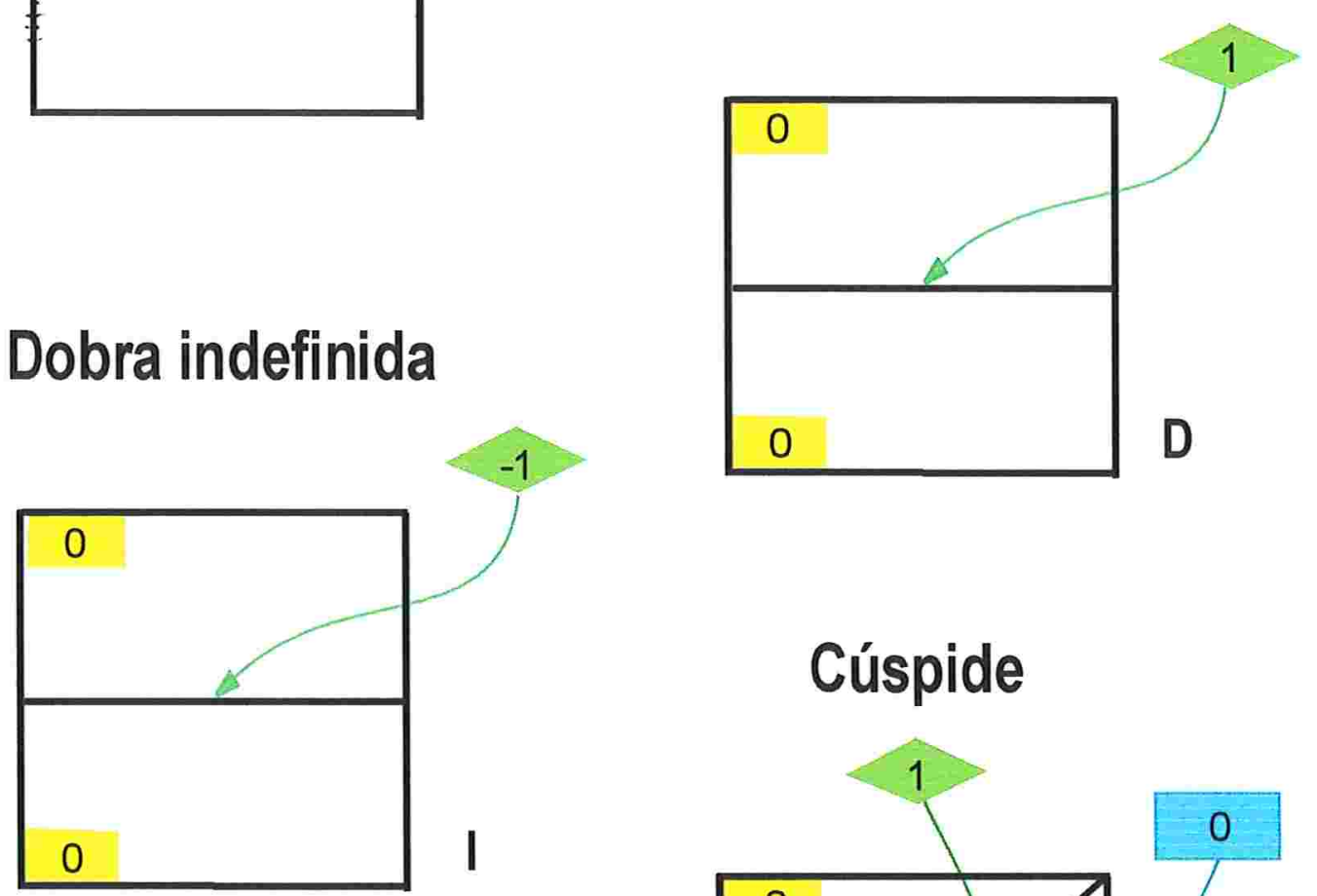

Cúspide

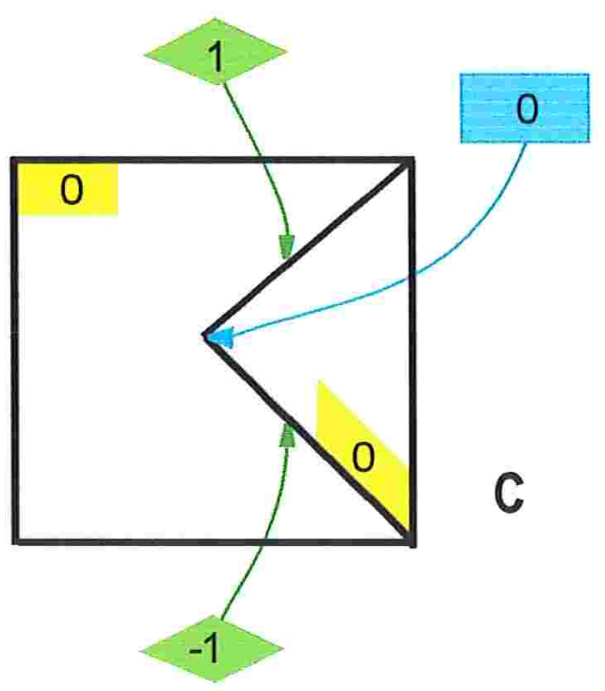




\section{Característica de Euler das fibras}

Dobra definida + definida

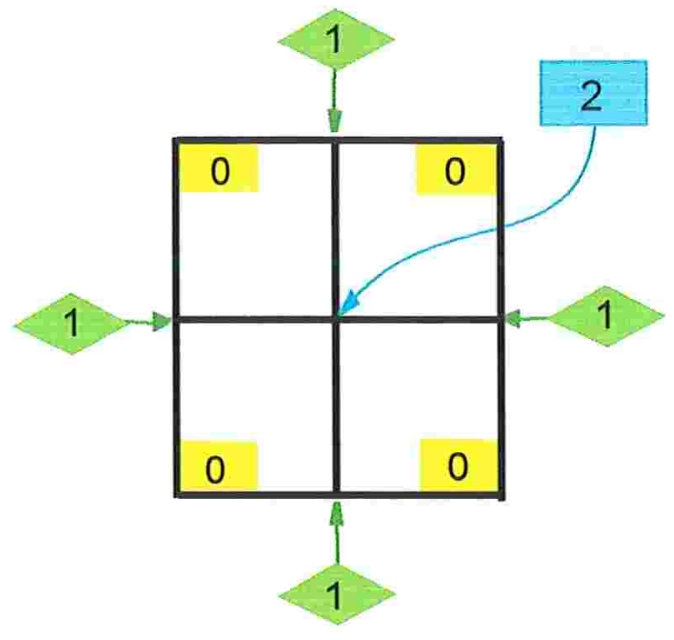

Dobra indefinida + indefinida
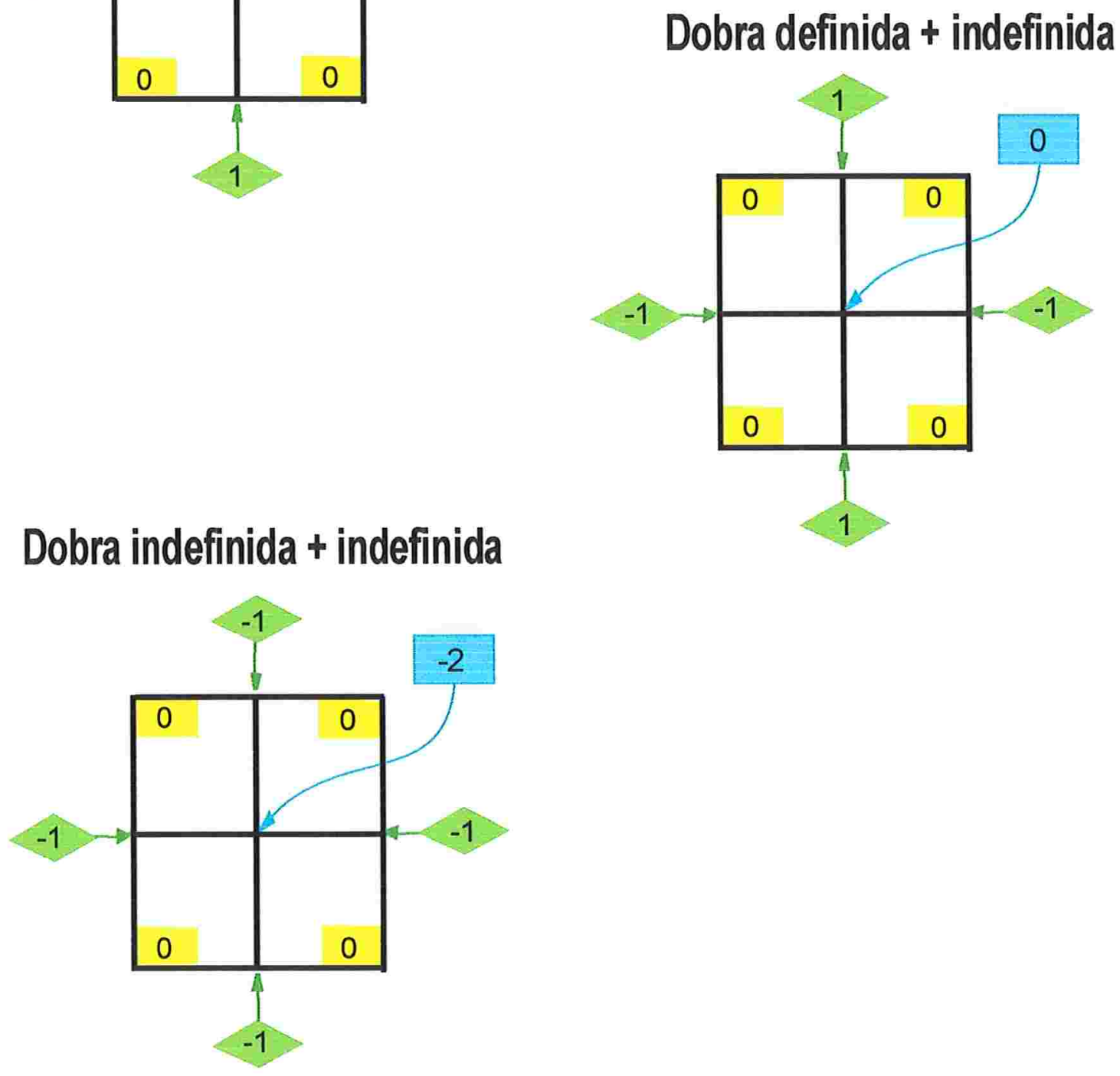


\section{Apêndice $\mathrm{C}$}

\section{As componentes conexas das fibras de $N^{4} \rightarrow \mathbb{R}^{3}$}

Nas figuras seguintes estão representadas as componentes das fibras.

Os retângulos azuis indicam as componentes das fibras dos pontos isolados T, CF ou $S W$.

Os losangos verdes indicam as componentes das fibras de pontos sobre as curvas $F F$ ou $C$.

Os retângulos amarelos indicam as componentes das fibras de pontos sobre as superfícies $D$ ou $I$.

As elipses vermelhas indicam as componentes das fibras de valores regulares. 
Componentes conexas das fibras
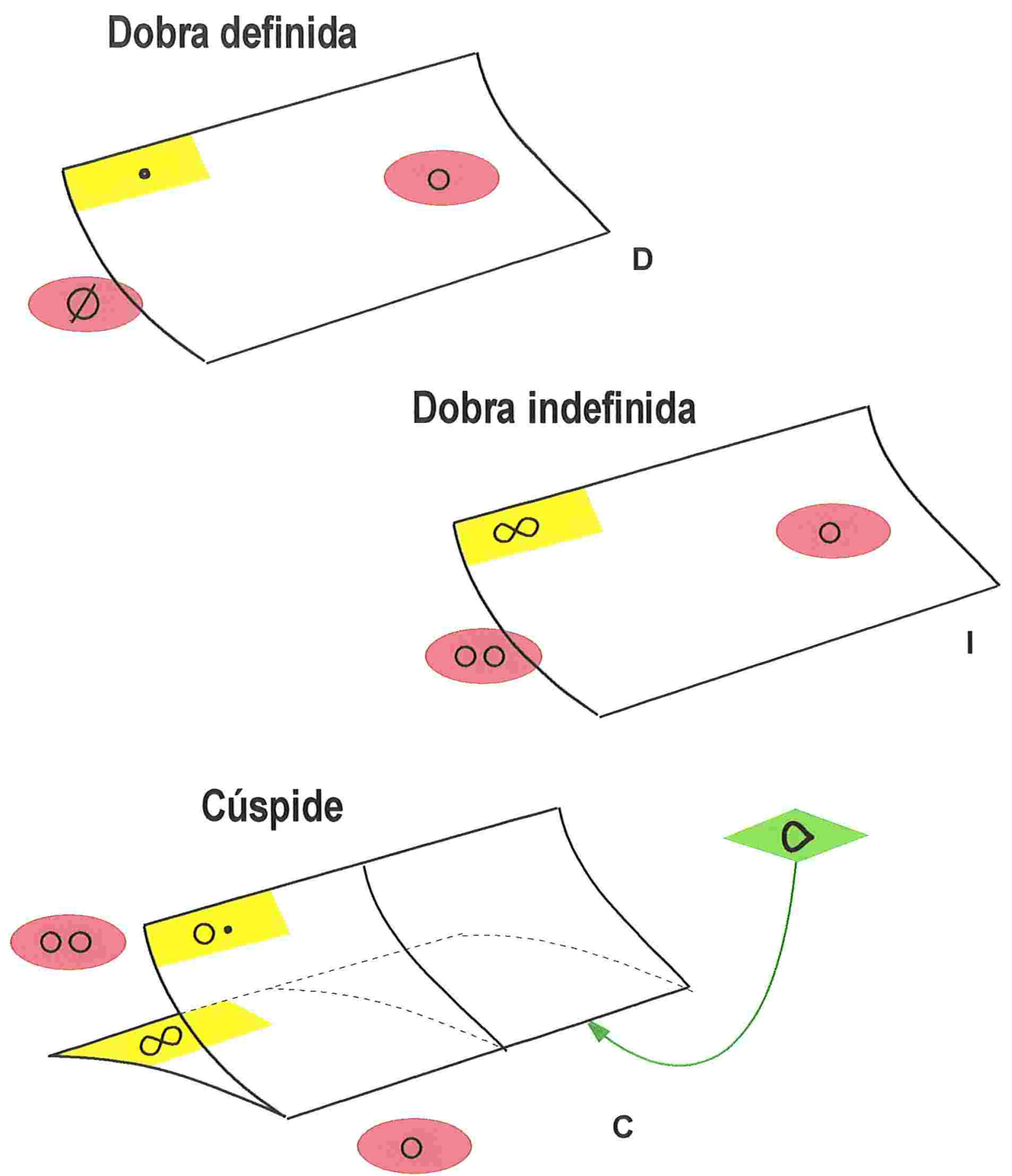
Componentes conexas das fibras:

Pontos duplos - (dobra + dobra)
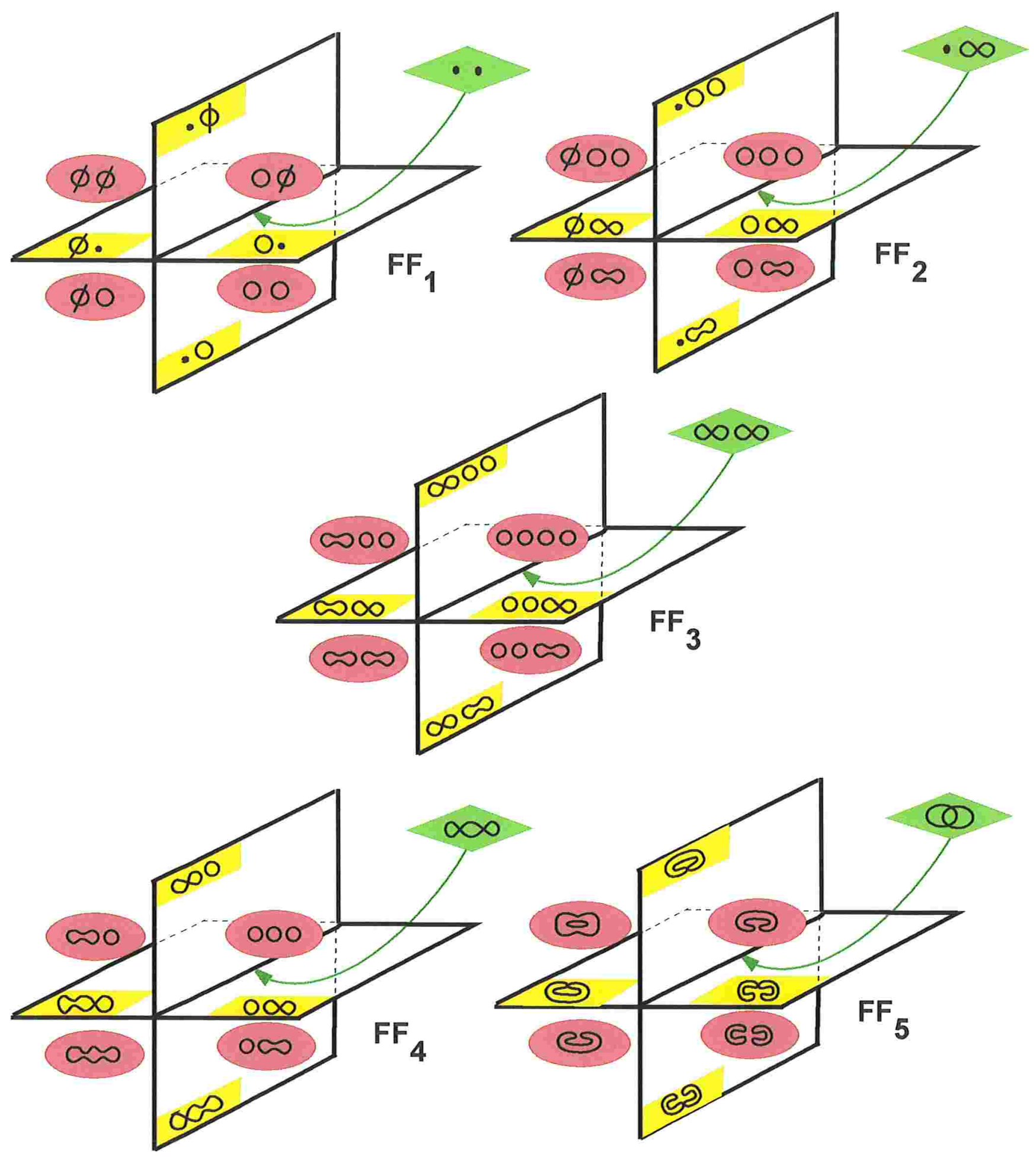


\section{Componentes conexas das fibras: Pontos triplos - 1}

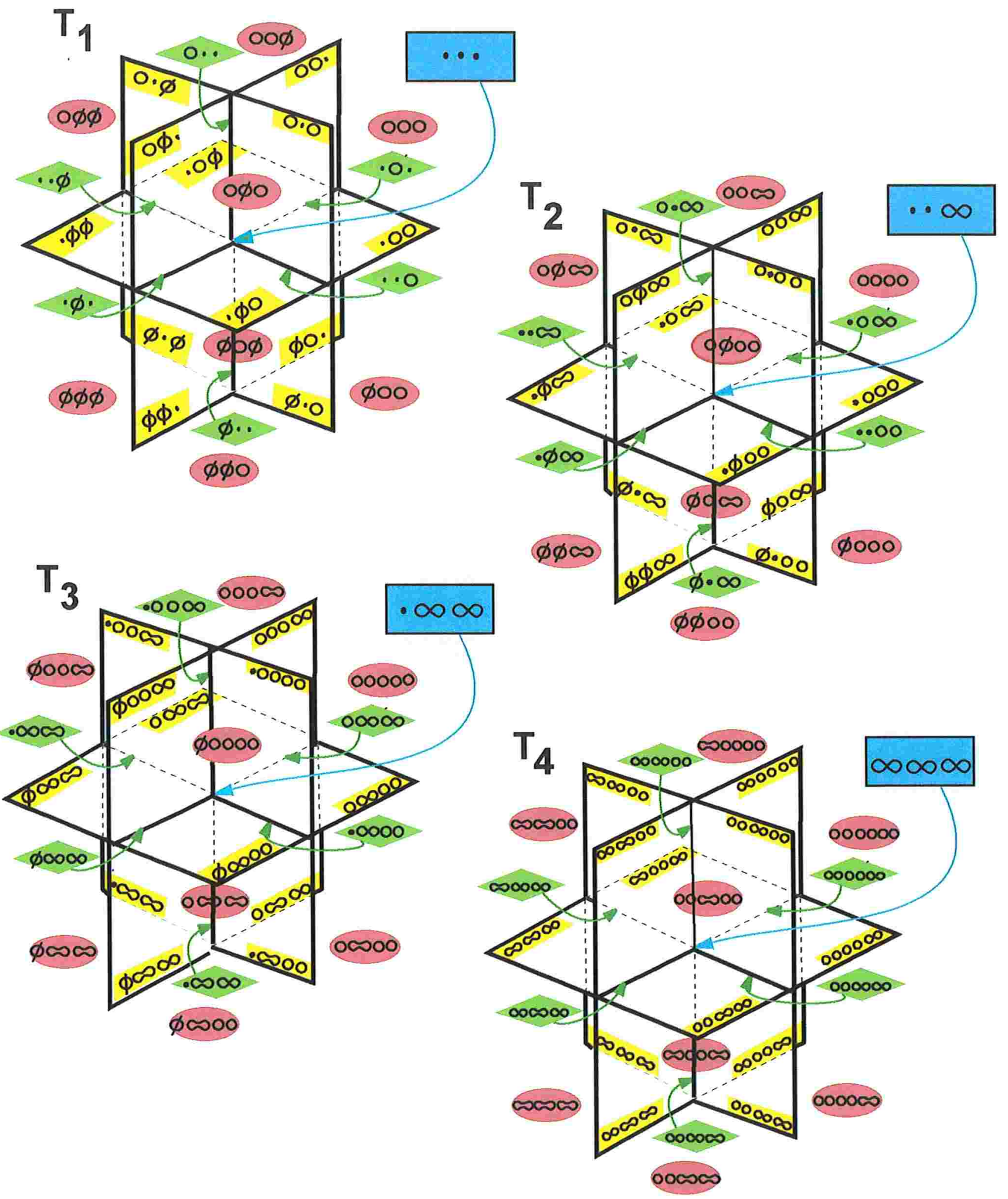


Componentes conexas das fibras:

Pontos triplos - 2
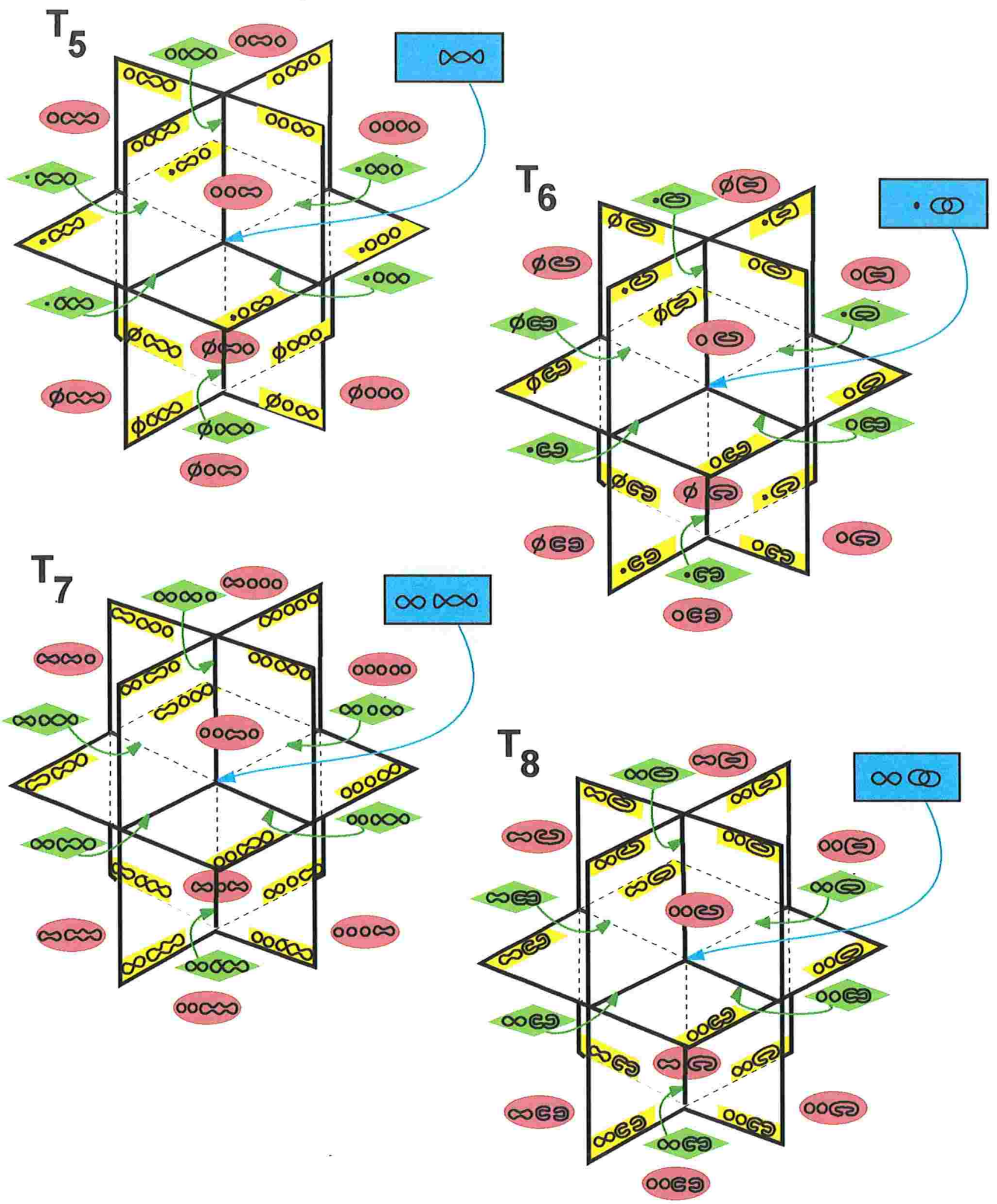
Componentes conexas das fibras:

Pontos triplos - 3

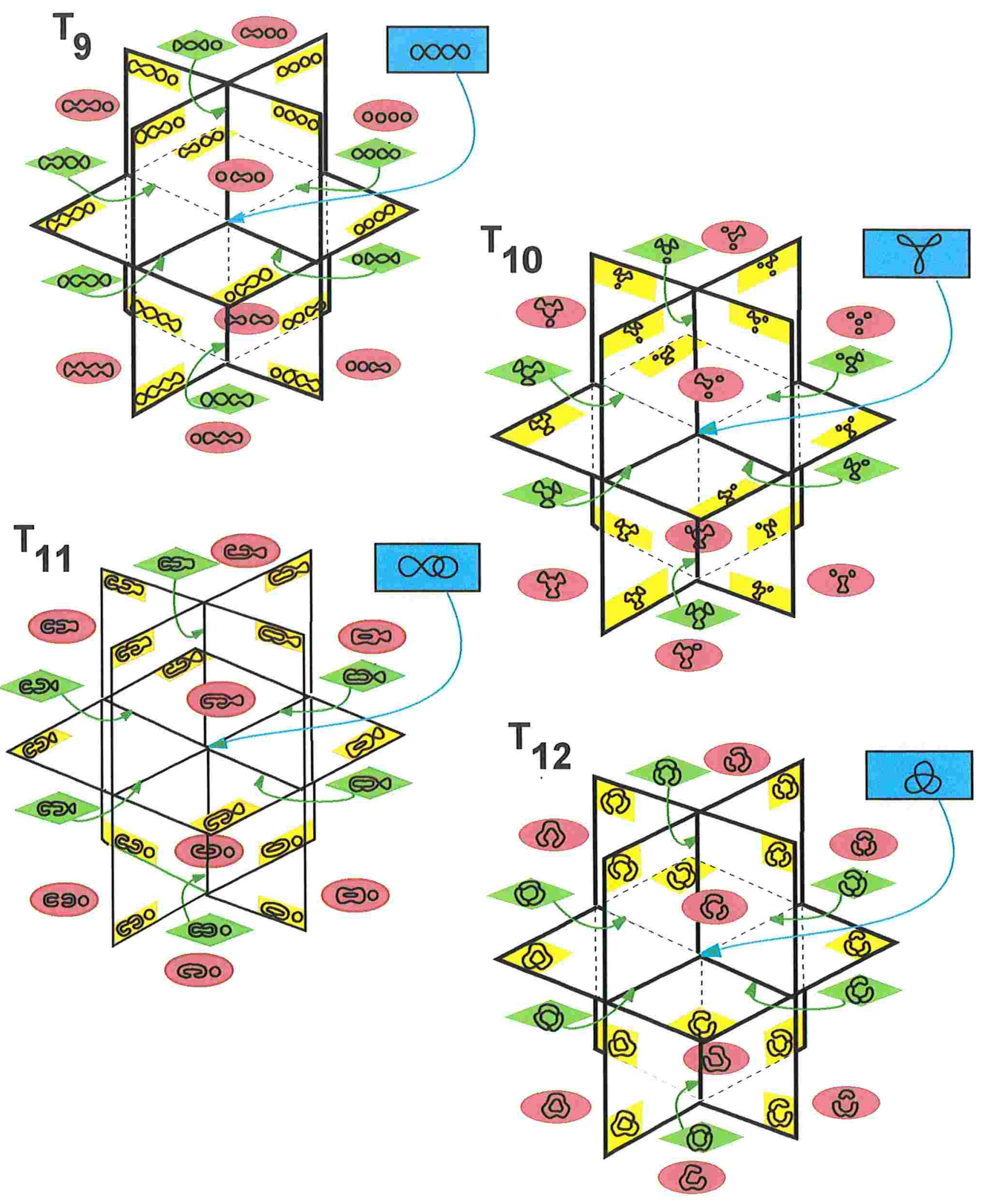


Componentes conexas das fibras:

Pontos triplos - 4

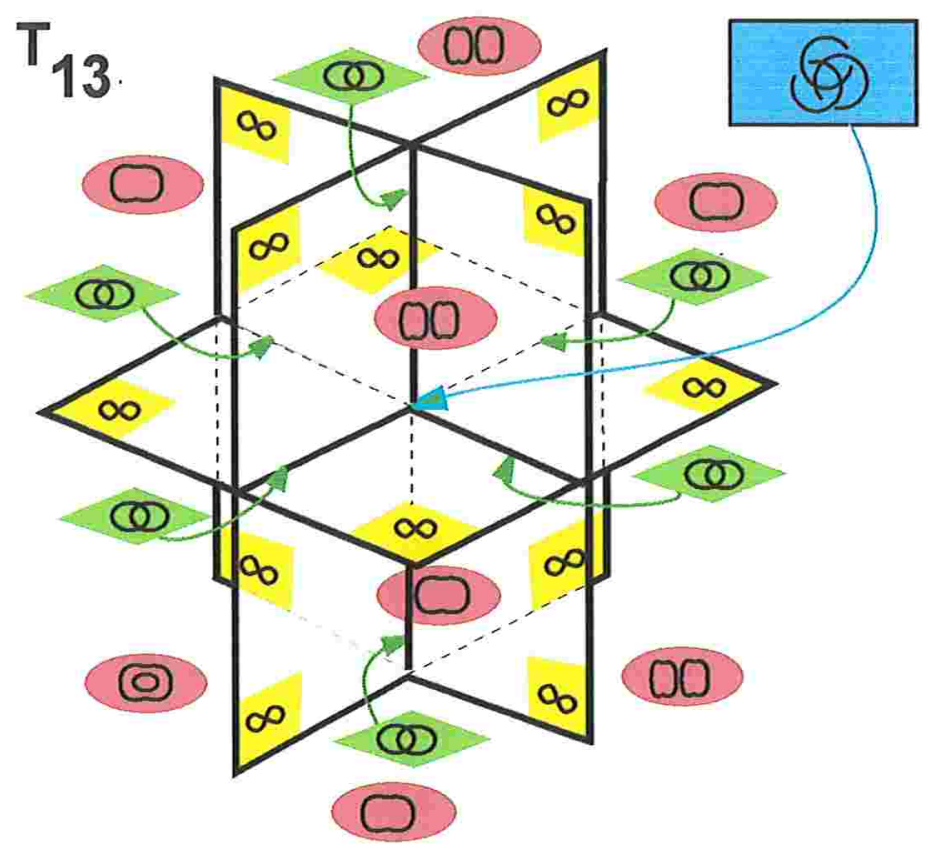




\section{Componentes conexas das fibras: Pontos duplos - (cúspide + dobra)}
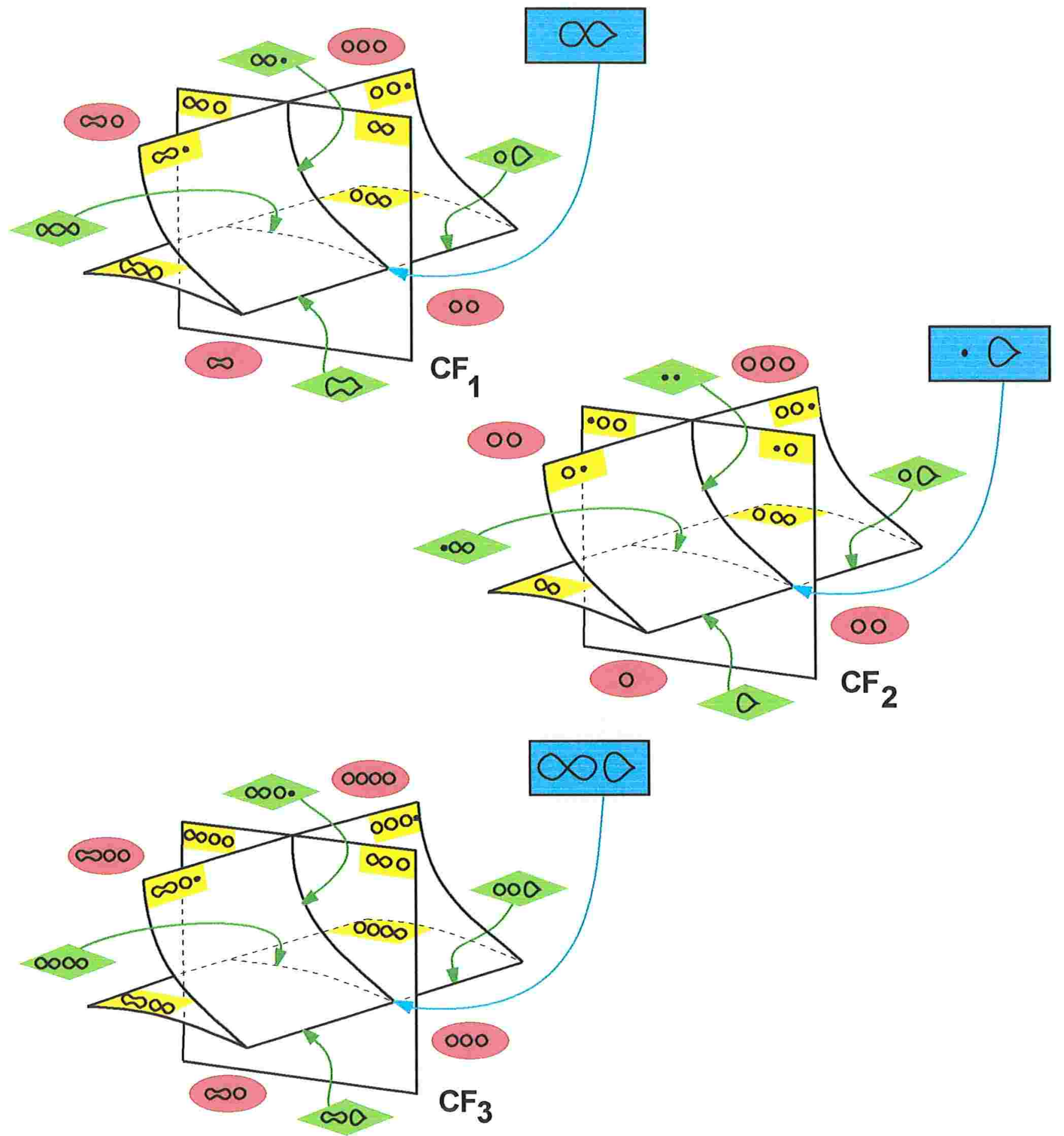
Componentes conexas das fibras:

Rabos de Andorinha
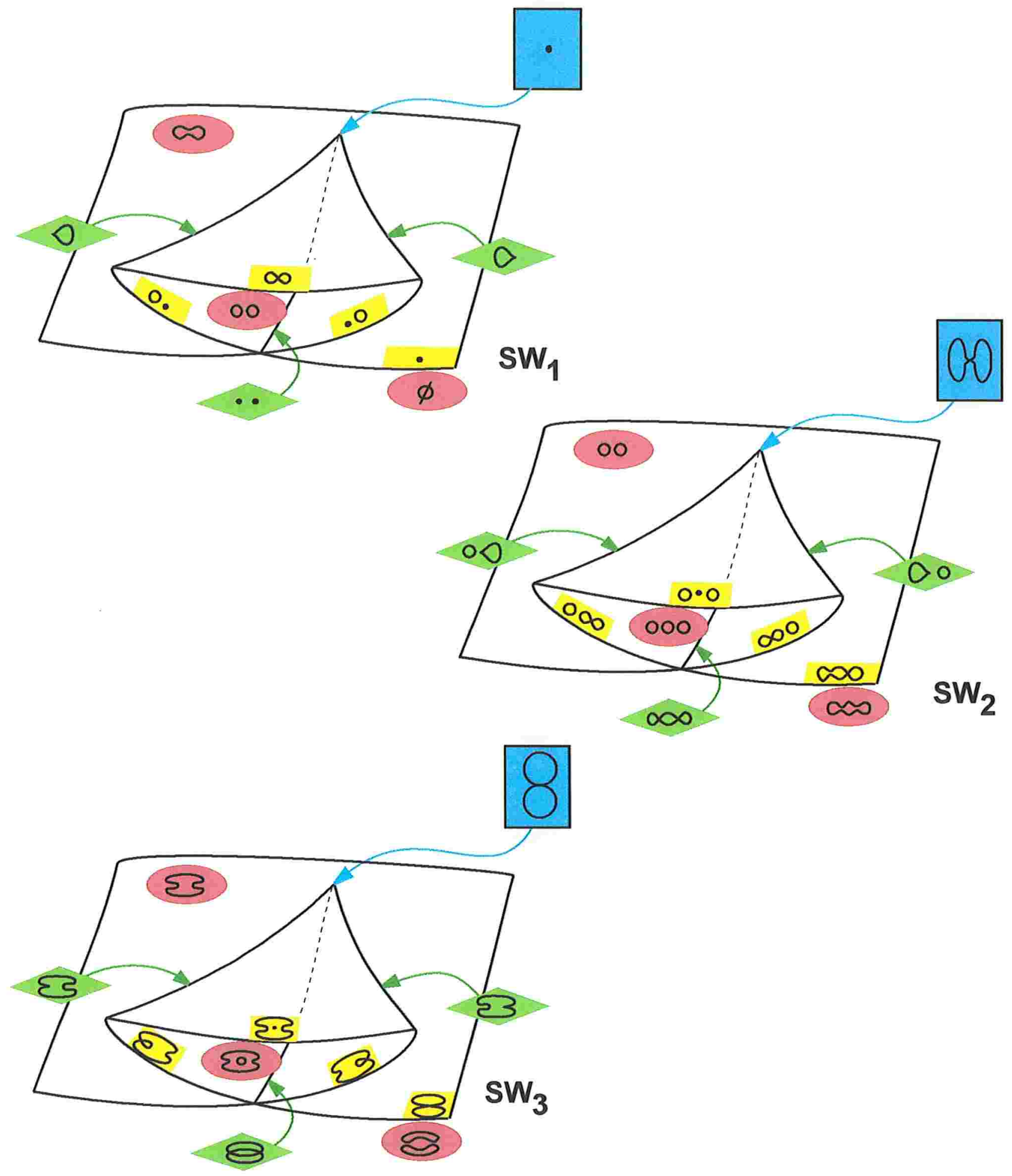


\section{Apêndice D}

\section{As características de Euler das fibras de $N^{4} \rightarrow \mathbb{R}^{3}$}

Os números, que aparecem nas figuras verdes e amarelas desta seção, indicam os valores das características de Euler das fibras sobre as regiões correspondentes que é necessário para o cálculo dos índices da Observação 6.3.3. 


\section{Característica de Euler das fibras}
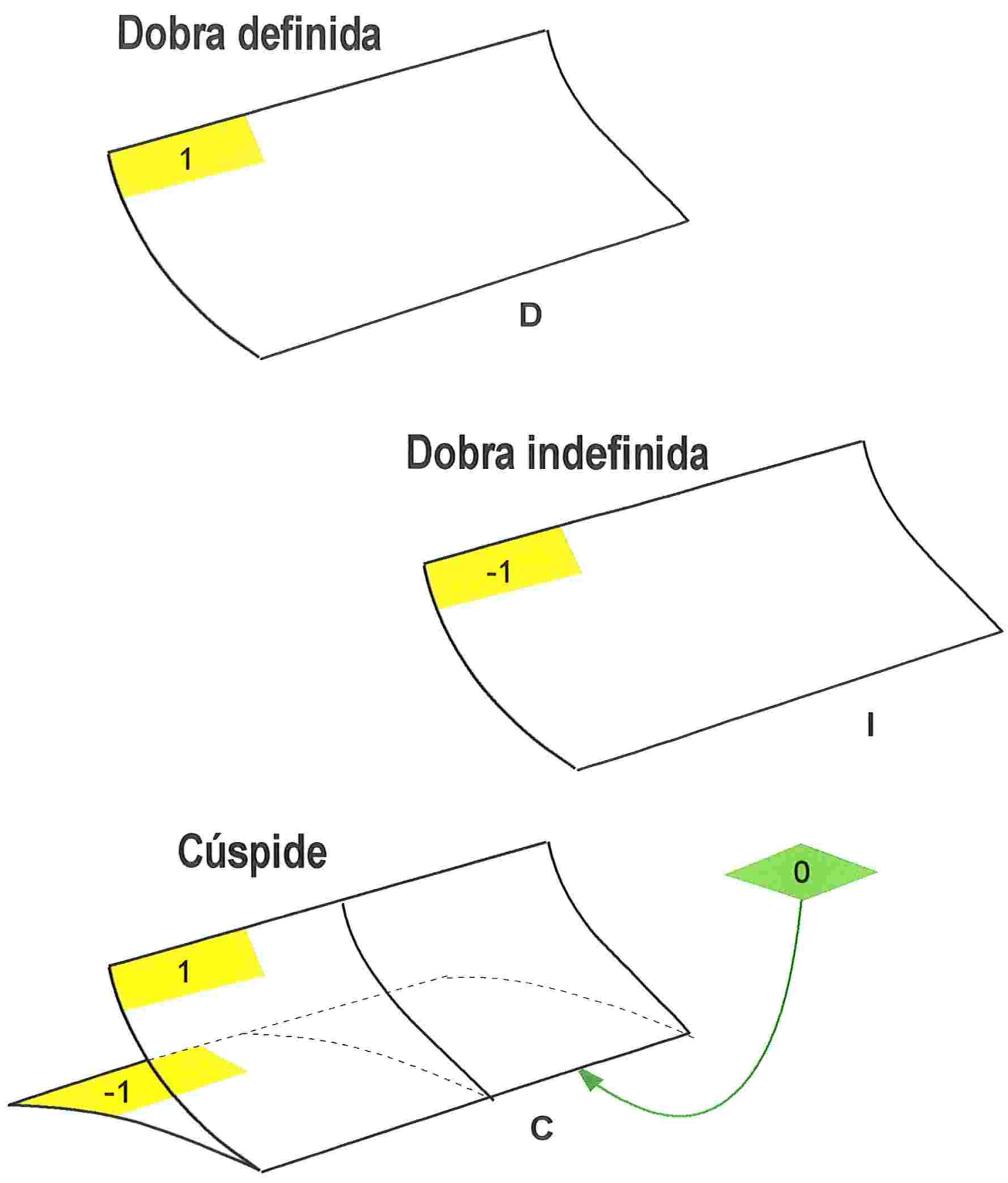
Característica de Euler das fibras:

Pontos duplos (dobra + dobra)
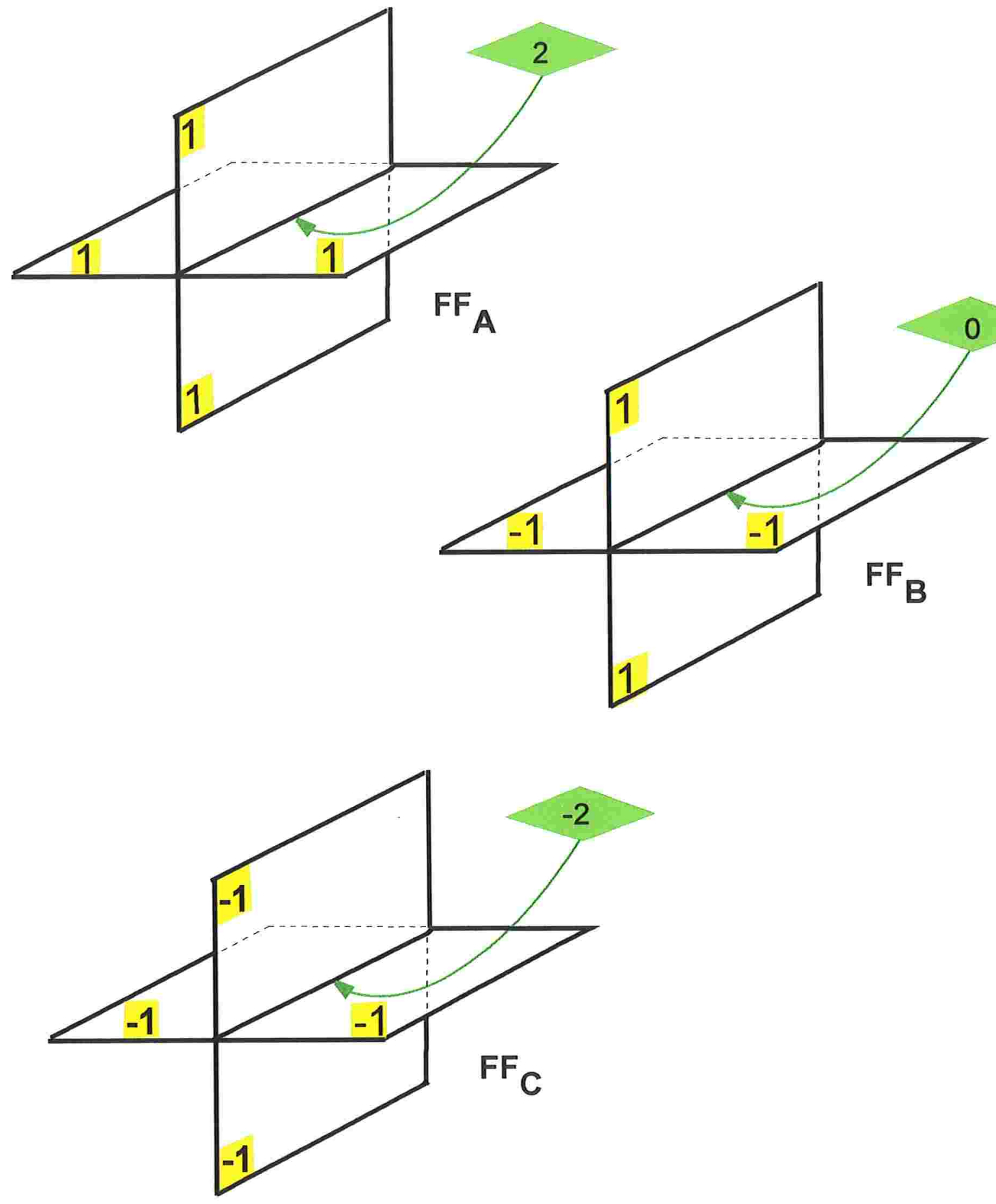
Característica de Euler das fibras:

Pontos triplos
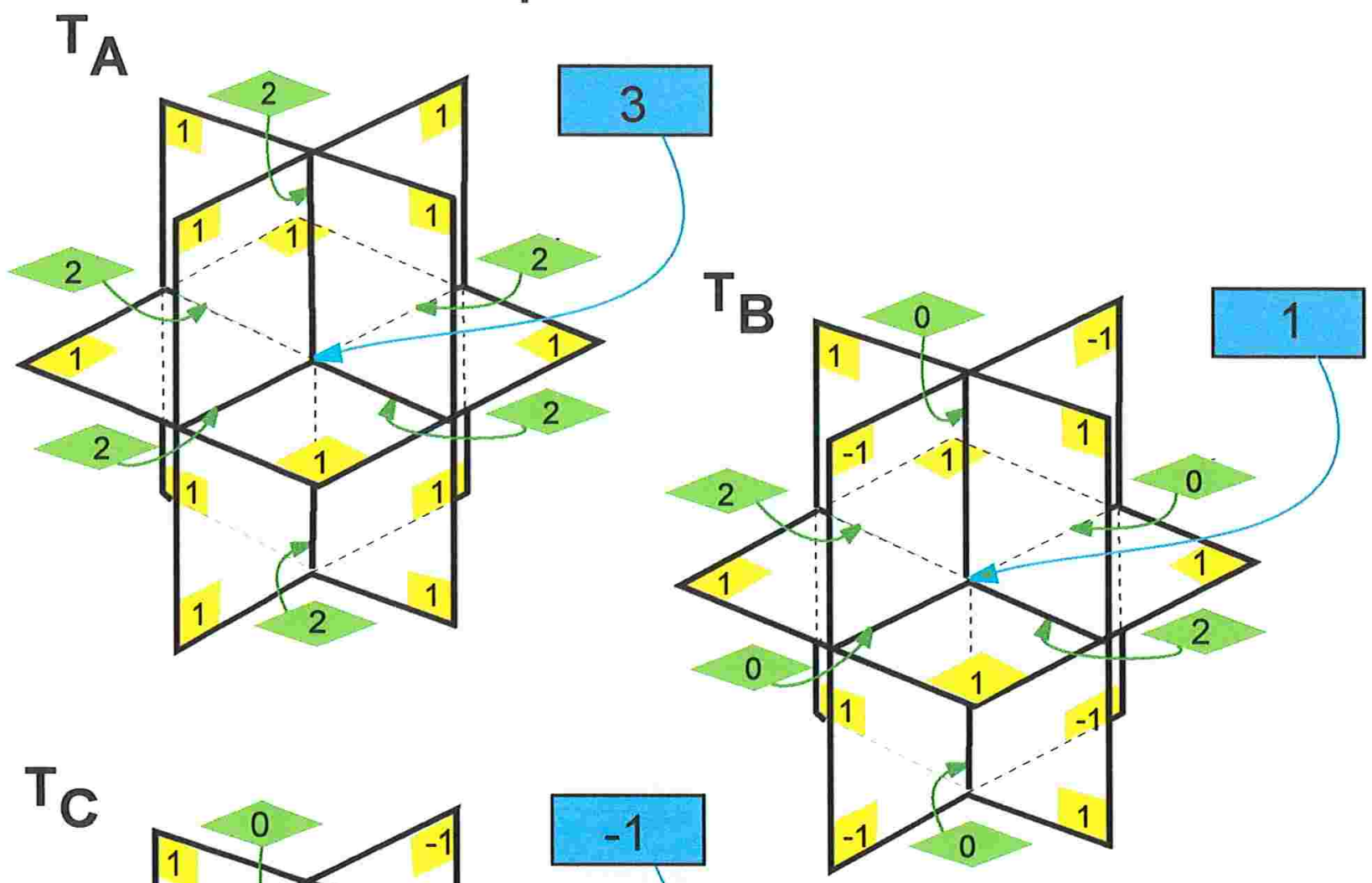

${ }^{\mathrm{T}} \mathrm{C}$
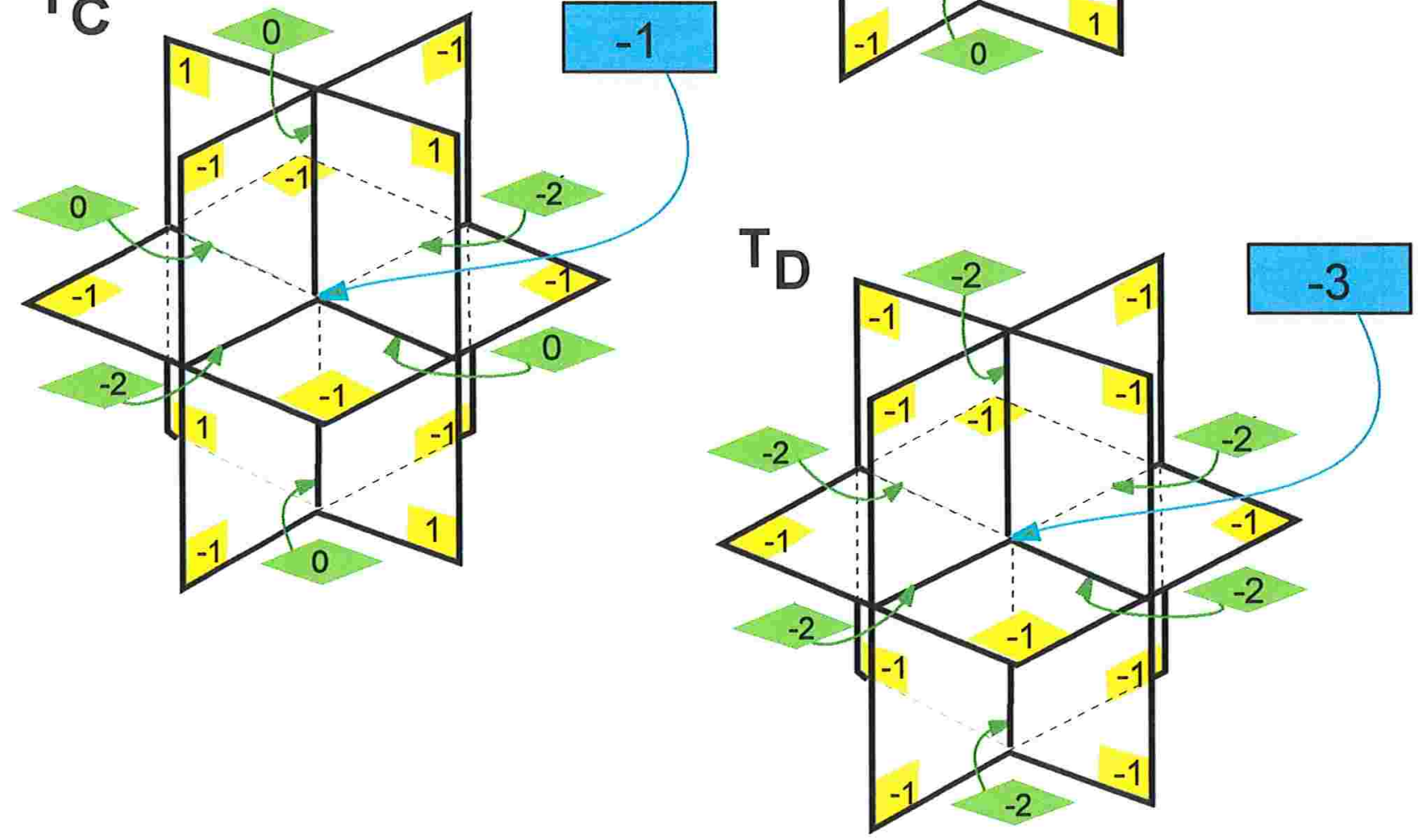
Característica de Euler das fibras:

Pontos duplos (dobra + cúspide)
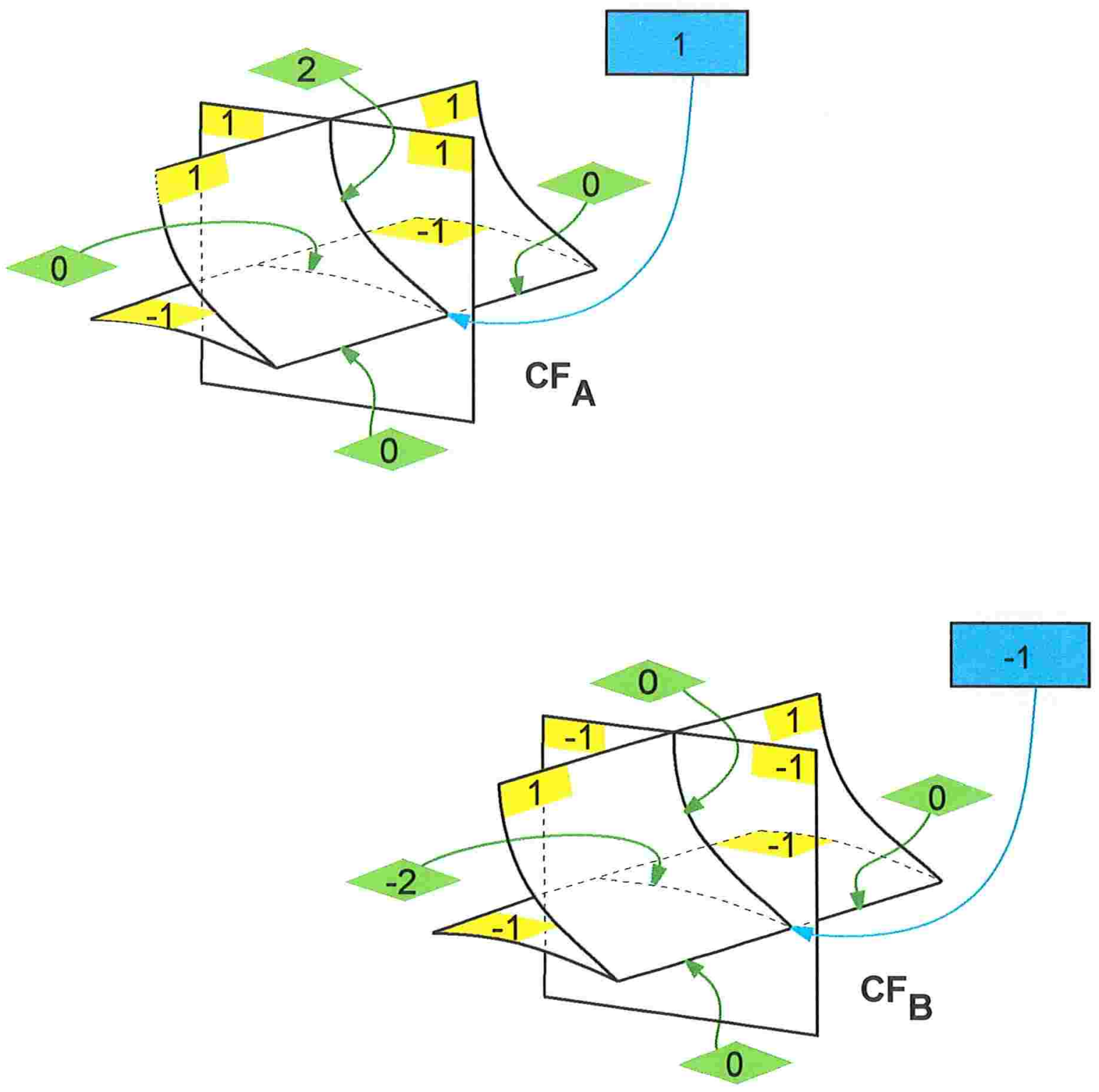
Característica de Euler das fibras:

Rabos de Andorinha
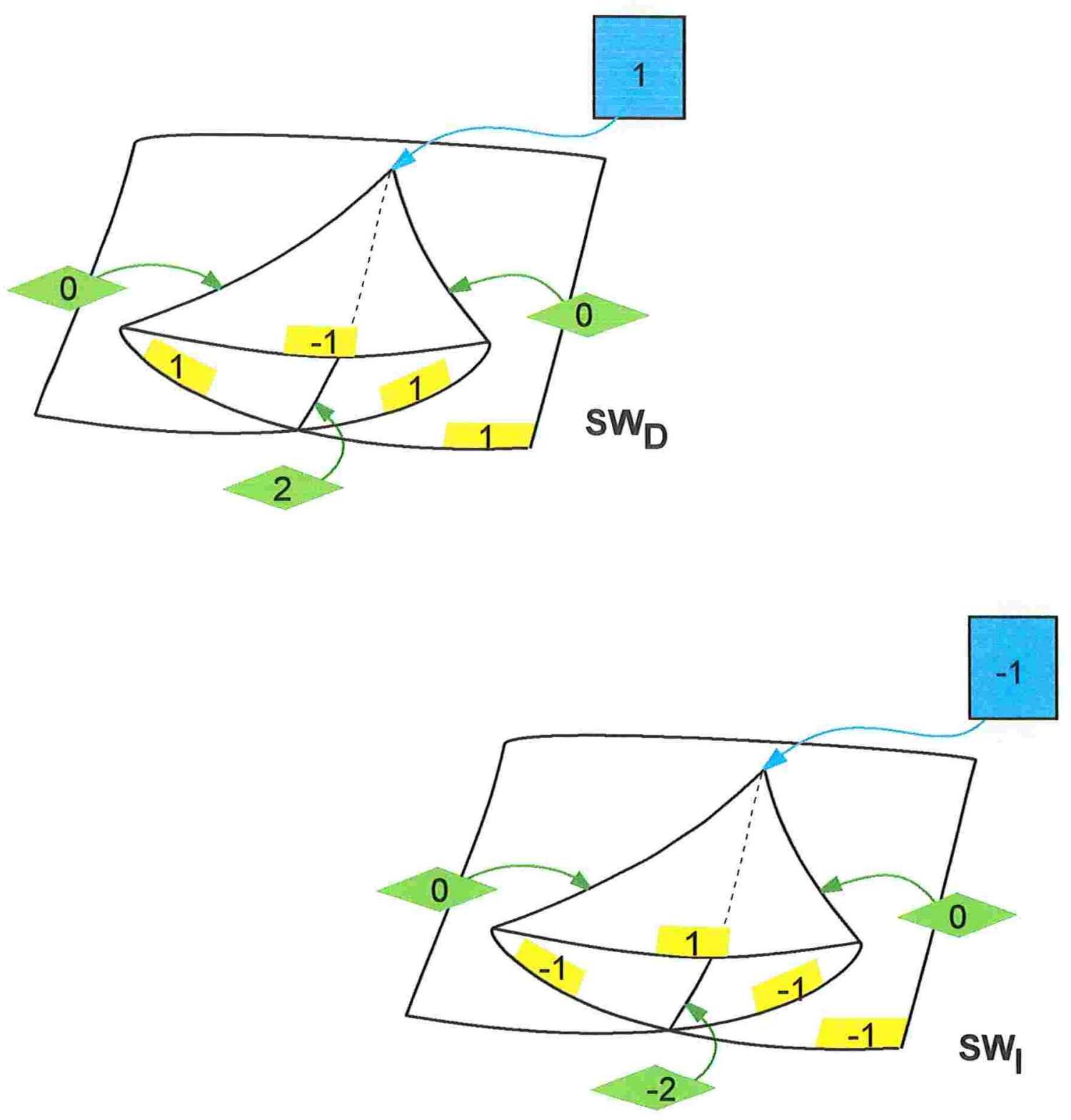


\section{Referências Bibliográficas}

[BS] J.J. Nuño Ballesteros \& O. Saeki, Euler Characteristic Formulas for Simplicial Maps, Math. Proc. Camb. Phil. Soc. 130 (2001), 307-331.

[Ba] T. F. Banchoff, Cusp Parity, unpublished, 1974.

[Br] G. E. Bredon, Topology and Geometry, Graduate Texts in Mathematics, Vol. 139, Springer-Verlag, 1993.

[BdR] O. Burlet \& G. de Rham, Sur Certaines Applications Génériques d'une Variété Close à Trois Dimensions dans le Plan, Enseign. Math. 20 (1974), 275-292.

[Fk] T. Fukuda, Topology of Folds, Cusps and Morin Singularities, In "A Fete of Topology", eds. Y. Matsumoto, T. Mizutani and S. Morita, Academic Press, 1987, pp.331-353.

[FI] T. Fukuda \& G. Ishikawa, On the Number of Cusps of Stable Perturbations of a Plane-to-Plane Singularity, Tokyo J. Math. 10 (1987), 375-384.

[Fr] Y. K. S. Furuya, Sobre Aplicą̧ões Genéricas $M^{4} \rightarrow \mathbb{R}^{2}$, Tese, I.C.M.S.C.-USP, São Carlos, Brasil, 1986.

[Ge1] A. Geloneze Neto, Cusp Parity and Generalized Riemann-Hurwitz Formulas for Simplicial Mappings, Ph.D. Thesis, Brown University, 1995. 
[Ge2] A. Geloneze Neto, A Generalized Riemann-Hurwitz Formula for Mappings on Surfaces, preprint, April 1997.

[GM] A. Geloneze Neto \& R. J. Morris, Combinatorics of Singularities in Dimension Three, preprint.

[GG] M. Golubitsky \& V. Guillemin, Stable Mappings and Their Singularities, Graduate Texts in Mathematics, Vol. 14, Springer-Verlag, 1973.

[Ho] K. Houston, Images of Finite Maps with One Dimmensional Double Point Set, Topology Appl. 91 (1999), 197-219.

[Hu] J. F. P. Hudson, Piecewise Linear Topology, W. A. Benjamin, Inc., New York, 1969.

[IM1] S. Izumiya \& W. L. Marar, The Euler Characteristic of a Generic Wavefront in a 3-Manifold, Proc. Amer. Math. Soc. 118, (1993), 13471350 .

[IM2] S. Izumiya \& W. L. Marar, On Topologically Stable Singular Surfaces in a 3-Manifold, J. Geom. 52 (1995), 108-119.

[IM3] S. Izumiya \& W. L. Marar, The Euler Characteristic of the Image of a Stable Mapping from a Closed n-Manifold to a $(2 n-1)$-Manifold, Workshop on Real and Complex Singularities (São Carlos, 1992), Mat. Contemp. 5 (1993), 53-59.

[KP] A. G. Khovanskii \& A. V. Pukhlikov, Integral Transforms Based on Euler Characteristic and Their Applications, Integral Transforms and Special Functions 1, (1993), 19-26.

[Ko] M. Kobayashi, Two Stable Maps of $\mathbb{C P}^{2}$ into $\mathbb{R}^{3}$, Memoirs of the College of Education, Akita Univ. Nat. Sci., 51 (1997), 5-12. 
[KS] M. Kobayashi \& O. Saeki, Simplifying Stable Mappings into the Plane from a Global Viewpoint, Trans. Amer. Math. Soc. 348 (1996), 26072636.

[KLP] L. Kushner, H. Levine \& P. Porto, Mapping Three-Manifolds into the Plane I, Boletin de la Sociedad Matemática Mexicana 29 (1984), 11-31.

[Le] H. Levine, Classifying Immersions into $\mathbb{R}^{4}$ over Stable Maps of 3Manifolds into $\mathbb{R}^{2}$, Lect. Notes in Math., Vol. 1157, Springer-Verlag, Berlin, New York, 1985.

[Li] E. L. Lima, Elementos de Topologia Geral, Livros Técnicos e Científicos Editora S.A., IMPA, 1976.

[ML] L. E. Mata-Lorenzo, The Stein Factorization for Stable Maps and Pistable Arcs of Maps from 3-Manifolds into the Plane, Ph.D. Thesis, Brandeis University, 1986.

[Ma] J. N. Mather, How to Stratify Mappings and Jet Spaces, Lect. Notes in Math., Vol. 535, Springer-Verlag, Berlin, New York, 1976, pp.128-176.

[Mi] J. W. Milnor, Topology from the Differentiable Viewpoint, The University Press of Virginia, 1965.

[MS] J. W. Milnor \& J. D. Stasheff, Characteristic Classes, Princeton University Press, 1974.

[MPS] W. Motta, P. Porto Jr \& O. Saeki, Stable Maps of 3-Manifolds into the Plane and Their Quotient Spaces, Proc. London Math. Soc. (3) 71 (1995), 158-174.

[Mu] J. R. Munkres, Elements of Algebraic Topology, The Benjamin/Cummings Publishing Company, Inc., 1984. 
[PF] P. Porto Jr \& Y. K. S. Furuya, On Special Generic Maps from a Closed Manifold into the Plane, Topology and its Applications 35 (1990), 41-52.

[Qu] J. R. Quine, A Global Theorem for Singularities of Maps Between Oriented 2-Manifolds, Trans. Amer. Math. Soc. 236 (1978), 307-314.

[Ro] J. J. Rotman, An Introduction to Algebraic Topology, Springer-Verlag, New York, 1988.

[RS] C. P. Rourke \& B. J. Sanderson, Introduction to Piecewise-Linear Topology, Springer-Verlag, Berlin, New York, 1972.

[Sa1] O. Saeki, Topology of Special Generic Maps of Manifolds into Euclidean Spaces, Topology and its Applications 49 (1993) 265-293.

[Sa2] O. Saeki, Studying the Topology of Morin Singulaties from a Global Viewpoint, Math. Proc. Camb. Phil. Soc. 117 (1995) 223-235.

[Sh] M. Shiota, Thom's Conjecture on Triangulations of Maps, Topology 39 (2000), 383-399.

[Th] R. Thom, Les Singularités des Applications Différentiables, Ann. Inst. Fourier 6 (1955-56), 43-57.

[Tu] A. W. Tucker, Branched and Folded Coverings, Bull. Amer. Math. Soc. 42 (1936), 859-862.

[Ve1] A. Verona, Les Applications Topologiquement Stables Sont Triangulables, C. R. Acad. Sc. Paris Série I Math. 296 (1983), 271-274.

[Ve2] A. Verona, Stratified Mappings - Structure and Triangulability, Lect. Notes in Math., Vol. 1102, Springer-Verlag, Berlin, New York, 1984. 
[Vi] J. W. Vick, Homology Theory, An Introduction to Algebraic Topology, Academic Press, Inc., New York, 1973.

[Vr] O. Y. Viro, Some Integral Calculus Based on Euler Characteristic, Lect. Notes in Math., Vol. 1346, Springer-Verlag, Berlin, New York, 1989, 127-138.

[Wh] H. Whitney, On Singularities of Mappings of Euclidean Spaces-I; Mappings of the Plane into the Plane, Ann. Math. 62 (1955), 374-410. 